\title{
System of delay differential equations with application in Dengue fever
}

\author{
Vanessa Steindorf \\ Thesis submitted in accordance with the requirements of \\ Institute of Mathematics and Statistics of the \\ University of São Paulo \\ to obtain the title of Doctor of Science
}

Program: Applied Mathematics

Professor: Dr. Sergio M. Oliva

During the development of this study the author has received financial support by the Coordenação de Aperfeiçoamento de Pessoal de Nível Superior - Brasil (CAPES) - Finance Code 001 and from LIAM Laboratory for Industrial and Applied Mathematics, Department of Mathematics and Statistics, York University-Canada

São Paulo, September 2019 



\title{
System of delay differential equations with application in Dengue fever
}

\author{
Esta versão da tese contém as correções e \\ alterações sugeridas pela Comissão Julgadora \\ durante a defesa da versão original do trabalho.
}

Comissão Julgadora:

- Prof. Dr. Sergio Oliva - IME-USP

- Prof. Dr. Jianhong Wu - LIAM-York University

- Prof. Dr. Norberto Anibal Maidana - CMCC-UFABC

- Prof. Dr. Claudia Pio Ferreira - IBB-UNESP

- Prof. Dr. Pedro da Silva Peixoto - IME-USP 



\section{Acknowledgements}

Gostaria de agradecer minha família, meu pai Jader, minha mãe Tercila e ao meu irmão Andrei que sempre se dispuseram a dar o melhor para mim e estavam, mesmo longe, na torcida para que eu alcançasse minhas conquistas. À minha irmã Andressa que me acompanhou em muitas aventuras e obstáculos da minha vida. Aos meus avós, Assunta e Doviglio, que sempre me quiseram por perto, mas entendiam as minhas escolhas. À minha tia Tere que sempre estava presente e torcendo para meu sucesso. Aos meus amigos da minha terra natal que sempre estiveram presentes em vários momentos.

Aos meus amigos da faculdade Rian Lopes, Rodrigo Gabert, Ronaldo Pes e Thiago Maboni por muitos momentos alegres e de descontração. Aos amigos que passaram e deixaram sua marca registrada nos momentos da minha vida, Lisiane Camana do 34, Lais, Jani e Roberta, do 32 e, às minhas companheiras e colegas de estágio Eliana Pinheiro e, Simone Franco. Um agradecimento enorme aos meus colegas, em especial, Jean Nakasato que sempre me ajudou e novamente, agradecer Rian que merece o título de melhor amigo, colega e agora, compadre, que junto com a comadre Mariele Almeida compartilhamos de vários momentos especiais. Ao meu namorado que presenciou todos meus obstáculos e dificuldades do doutorado e, sua família com seu apoio incondicional. Às minhas colegas de apartamento Cristina, Raquel e as meninas com as quais pude compartilhar inesquecíveis momentos: Jéssica e Caroline Vaitkevicius.

Aos professores de dança que me ensinaram Forró; Roberto e Daniel. Aos meus professores de Zouk; Marina Tuji, José, Fernando, Mari Inoue e Vitor Trivilin e, de Sertanejo; Bia, Renan, Murilo e Letícia. Finalmente aos meus amigos e colegas da dança e dos grupos Polidance e Nárnia os quais tive o prazer de compartilhar muita diversão e alegrias. Por fim, às 
minhas amigas do ABC, Marina, Tuji e Karina e aos amigos da UFABC.

As minhas colegas e amigas ao qual tive o prazer de ter deliciosos almoços, conversas e risadas, Bruna Santos, Luana Bassani, Jerusa Megale, Ana Rojas, Larissa Sartori, Simone Brito, Pollyana Vicente, Gregório Luís, Leonardo Poveda Cuevas e Hugo Ribeiro. Ao meu colega Fernando Ravelo que se colocou a disposição, discutindo e me auxiliando na otimização dos programas do Matlab. Aos meus professores da graduação Thaisa Miotto, Marcio Miotto e João Lazzarin que me fizeram impulsionar e acreditar nesta carreira.

Agradeço ao meu orientador de mestrado Dr. Norberto Maidana e ao meu orientador de doutorado Dr. Sergio Oliva. Agradeço aos professores Dr. Eduardo Massad, Marcelo Burattini e Pedro da Silva Peixoto pelas produtivas discussões do trabalho.

I am grateful for the attention, help and great dinners with Dr. Xi Huo, Dr. Kyeongah Nah, Xiaomei Feng, Biao Tang, Mahnaz Alavinejad and Xiaotian Wu, in Toronto, during my interchange. Also, I am grateful for the kind invitation made by Dr. Jianhong Wu in order to go York University to do the joint research which has greatly improved this work.

Finalmente, agradeço a banca por aceitar este convite. E as pessoas que não citei, que direta ou indiretamente fizeram parte da minha formação, e fizeram parte desta minha trajetória, o meu muito obrigado. 


\section{Abstract}

Dengue fever is endemic in tropical and sub-tropical countries, and some of the important features of Dengue fever spread continue posing challenges for mathematical modelling. We propose a model, namely a system of integro-differential equations, to study a multi-serotype infectious disease. The main purpose is to include and analyse the effect of a general time delay on the model describing the length of the cross immunity protection and the effect of Antibody Dependent Enhancement (ADE), both characteristics of Dengue fever. Analysing the system, we could find the equilibriums in the invariant region. A coexistence endemic equilibrium within the region was proved, even for the asymmetric case. The local stability for the disease free equilibrium and for the boundary endemic equilibriums were proved. We have also results about the stability of the solutions of the system, that is completely determined by the Basic Reproduction Number and by the Invasion Reproduction Number, defined mathematically, as a threshold value for stability. The global dynamics is investigated by constructing suitable Lyapunov functions. Bifurcations structure and the solutions of the system were shown through numerical analysis indicating oscillatory dynamics for specific value of the parameter representing the $\mathrm{ADE}$. The analytical results prove the instability of the coexistence endemic equilibrium, showing complex dynamics. Finally, mortality due to the disease is added to the original system. Analysis and discussions are made for this model as perturbation of the original non-linear system.

Keywords: distributed delay, multi-strain model, temporary immunity, antibody dependent enhancement - ADE, integro-differential equations. 



\section{Resumo}

A Dengue é endêmica em países tropicais e subtropicais e, algumas das importantes características da dengue continua sendo um desafio para a modelagem da propagação da doença. Assim, propomos um modelo, um sistema de equações integro-diferenciais, com o objetivo de estudar uma doença infecciosa identificada por vários sorotipos. O principal objetivo é incluir e analisar o efeito de um tempo geral de retardo no modelo descrevendo o tempo de imunidade cruzada para a doença e o efeito do Antibody Dependent Enhancement (ADE). Analisando o sistema, encontramos os equilíbrios, onde a existência do equilíbrio de coexistência foi provado, mesmo para o caso assimétrico. A estabilidade local para o equilíbrio livre de doença e para os equilíbrios específicos de cada sorotipo foi provada. Também mostramos resultados para a estabilidade das soluções do sistema que é completamente determinada pelo Número Básico de Reprodução e pelo Número Básico de Invasão, definido matematicamente como um valor limiar para a estabilidade. A dinâmica global é investigada construindo funções de Lyapunov. Adicionalmente, bifurcações e as soluções do sistema foram mostrados via análise numérica indicando dinâmica oscilatória para específicos valores do parâmetro que representa o efeito ADE. Resultados analíticos obtidos pela teoria da perturbação provam a instabilidade do equilíbrio endêmico de coexistência e apontam para um complexo comportamento do sistema. Por fim, a mortalidade causada pela doença é adicionada ao sistema original. Análises e discussões são feitas para este modelo como uma perturbação do sistema não linear original.

Palavras-chave: retardo distribuído, modelo de multi sorotipos, imunidade temporária cruzada, antibody dependent enhancement (ADE), equações integro-diferenciais. 



\section{Table of Contents}

1 Introduction 1

1.1 Theoretical Background . . . . . . . . . . . . . . . . . . 3

1.2 Model Formulation and Application $\ldots \ldots \ldots$. . . . . . . . . 6

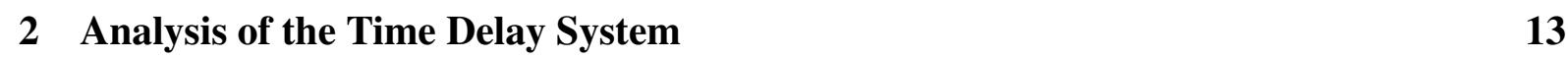

2.1 Limiting System . . . . . . . . . . . . . . . . . . . . . 13

$2.1 .1 \quad$ Basic Reproduction Number . . . . . . . . . . . . . . . . . 19

2.1 .2 Equilibriums of the Limiting System . . . . . . . . . . . . . . 20

2.1 .3 Symmetric Case ........................... 21

2.2 Stability Analysis . . . . . . . . . . . . . . . . . . . 23

2.2 .1 Stability of the Equilibriums of the Limiting System . . . . . . . . . . 24

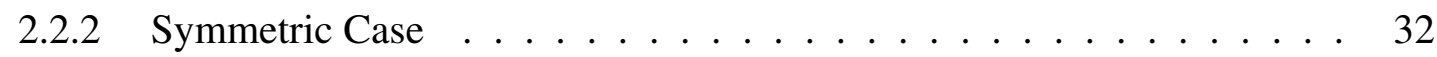

2.2 .3 Stability of the solutions of the Time Delay System . . . . . . . . . . 34

2.3 Global Stability . . . . . . . . . . . . . . . . . . . 35

2.3 .1 Global Stability of the DFE . . . . . . . . . . . . 35

2.3 .2 Global Stability of the Boundary Equilibrium . . . . . . . . . . . 37

\begin{tabular}{|lll}
\hline 3 & Numerical Analysis & 41
\end{tabular}

3.1 Stability of the Coexistence Endemic equilibrium . . . . . . . . . . . . 44

$3.1 .1 \quad$ Symmetric case . . . . . . . . . . . . . . . . . . . 44

3.1 .2 Asymmetric case . . . . . . . . . . . . . . . 46

$3.1 .3 \quad$ Bifurcation Structure $\ldots \ldots \ldots$. . . . . . . . . . . . . . . . . . . . . 49 
3.1 .4 Solutions of the system $\ldots \ldots \ldots \ldots$. . . . . . . . . . 50

3.2 Main Results . . . . . . . . . . . . . . . . . . . . . . . . . . . . . . . 59

4 Analysis of the Coexistence Endemic Equilibrium 61

$4.1 \quad$ Stability Analysis of the Endemic Equilibrium of the Time Delay System . . . 61

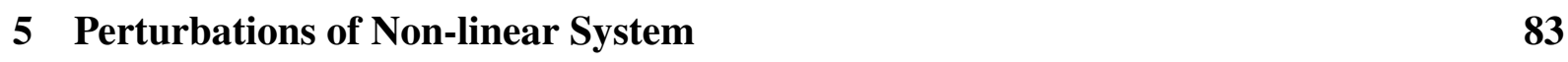

5.1 Adding mortality caused by disease $\ldots \ldots \ldots$. . . . . . . . . 83

5.2 Numerical Solutions of Long-term Behaviour $\ldots \ldots \ldots$. . . . . . . . . . 89

$\begin{array}{lll}6 & \text { Particular case } & 93\end{array}$

6.1 ODE System Analysis: Equilibriums of the System . . . . . . . . . . . . . 93

6.1 .1 Symmetric Case . . . . . . . . . . . . . . . . . 96

6.2 Stability Analysis of the Equilibriums $\ldots \ldots \ldots$. . . . . . . . . 98

$6.2 .1 \quad$ Basic Reproduction Number . . . . . . . . . . . . . . . . . . . 98

$6.2 .2 \quad$ Local Stability $\ldots \ldots \ldots$. . . . . . . . . . . . . . . . . 99

6.2 .3 Symmetric Case . . . . . . . . . . . . . . . 103

6.3 Numerical analysis: Particular case $\left(e^{-\omega_{i} t}\right) \ldots \ldots \ldots$. . . . . . . . 105

6.3.1 Stability of the Coexistence Endemic equilibrium . . . . . . . . . . . 105

6.3 .2 Solutions of the system $\ldots \ldots \ldots$. . . . . . . . . . . 112

6.4 Main Results . . . . . . . . . . . . . . . . . . . . . . . . . . . 121

6.5 Local Analysis of the Endemic Equilibrium of the ODE System . . . . . . . 122

$\begin{array}{lll}7 & \text { Discussion and Conclusion } & 133\end{array}$

7.1 Some Important Considerations $\ldots \ldots \ldots$

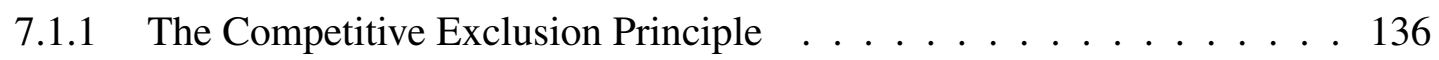

7.1 .2 The Invasion Reproduction Number . . . . . . . . . . . . . . . . . . 137

7.2 Final Conclusions $\ldots \ldots \ldots \ldots$. . . . . . . . . . . . . . . 137

\begin{tabular}{ll}
\hline Bibliography & 145
\end{tabular} 
CHAPTER 1

\section{Introduction}

Dengue fever is an endemic disease in tropical and sub-tropical countries. According to World Health Organization WHO (2018), in 2016, more than 2.38 million cases were reported in the Americas, where Brazil alone contributed with almost 1.5 million cases. In 2017, a significant reduction in the number of cases in the Americas was reported, even though a recent estimate indicates 390 million cases per year around the world (WHO, 2018).

Dengue fever constitutes a public health problem and it is caused by Dengue virus (DENV), which is transmitted to a human population by the bite of infected mosquitos. So far, it is known that there are four distinct virus serotypes circulating in the population (Gubler, 2014). A fifth variant (DENV-5) was discovered and isolated in October 2013, according to Mustafa (2015), however it just follows wild cycle.

The disease is presented in two forms: asymptomatic or symptomatic form. The symptomatic form can be presented as Dengue classic or Dengue hemorrhagic fever (DHF). Antibody-Dependent Enhancement (ADE) was proposed to explain the more frequent occurrence of severity of Dengue fever and the Dengue hemorrhagic fever in secondary infections (Guzman, 2013; Gubler, 2014). ADE happens when the antibodies acquired in a previous infection fail in neutralizing the next distinct virus, favouring the increasing of the viral replication (Guzman, 2013; Reich, 2013).

Another characteristic of Dengue virus is the cross immunity protection. It happens when the infection with any of the four dengue serotypes leads to a short term protection for all 
serotypes. Long term protection occurs only for the serotype which the individual was infected (Reich, 2013).

Motivated by the growing discussion about Dengue fever, since it is a public health problem, we proposed a mathematical model, namely, a system of Integro-Differential Equations (IDE) that can be applied to describe and to study the propagation of Dengue fever in a population, considering two main characteristics of the disease: ADE and cross immunity protection. The main purpose is to include and analyse the effect of the general time delay on the model, which is described to represent the length of the cross immunity protection, as observed in Dengue fever. In addition, a constant parameter will be added in the model in favour of studying the effect of ADE.

In the next section, concerning to summarize the mathematical theoretical framework and models that have been proposed to describe Dengue propagation, we are going to provide a brief description of the main studies and the main results. After that, we are going to propose the model.

The analysis of the time delay system is left for the second chapter, where we define and analyse the limiting system as well as look for equilibriums and show local stability. Subsequently, we finish the second section with results of the stability of solutions of the delay system. Afterwards, the last section of the third chapter is reserved to construct Lyapunov function, in order to prove the global stability.

Numerical analysis will be made at chapter three, where the bifurcation structure and stability of the coexistence equilibrium are numerically studied. At chapter four we prove analytically the instability of the endemic equilibrium using perturbation theory.

At chapter five, by adding a mortality term which represents small mortality due to the disease, we present the improvement of the original system and we analyse it through perturbation theory of non-linear systems.

At chapter six we are going to study a particular case of the model, as well as to perform the qualitative analysis of the ODE model, describing the equilibriums of the system. Also, we are going to show results about the local stability of the equilibriums, defining a threshold value which is very important for the stability. Finally, comparison of the results, important remarks, final considerations and conclusions will be made over the last chapter. 


\subsection{Theoretical Background}

Since Dengue virus remains a public health problem, a lot of researches have been done about it. The first work in dengue modelling including the ADE effect and multi-strains was done by Ferguson (1999a). In the paper, it has modelled the ADE consequences, where previous exposure to one serotype increases the transmission probability for the second infection. The Basic Reproduction Number was found, and it has explored two strains of dengue virus in the simple model.

Johansson (2011), concerned about the impact of Dengue vaccine, previously made a comparison table with the main studies in Dengue fever, from the first work in 1970 by Fischer until 2010 with Otero. The comparison table contained the differences among models, like number of serotypes included in the model, if it were described the vector population, and if $\mathrm{ADE}$ effect and cross protection were modelled. This way the author could validate and parametrize the models in order to evaluate the impact of immunization programs.

Another author made a review of mathematical studies in dengue transmission. Andraud (2012) reviewed articles, discussed and explored these studies based on epidemiological assumptions with focus on the impact of vaccination and also in relation to the impact of vector control.

Some more sophisticated models were proposed by Adams (2006), Bianco (2009), $\mathrm{Hu}$ (2013). Adams (2006) included the ADE effect in their two-strain model, he also described the immunological distance among dengue serotypes through a function with the hypotheses that this function reduces the probability of contracting a secondary infection. Numerical results were presented in that study.

Bianco (2009) proposed a mathematical model and evaluated the parameters that describe the ADE effect and cross immunity in the system showing that weak temporary cross immunity stabilizes the system, but strong cross immunity destabilizes the dynamics. Endemic steady state, reproduction number and the predicted location of the Hopf bifurcation were written assuming symmetry among all the strains. Numerical simulations were observed in order to analyse the impact of different interaction in a multi-strain model.

$\mathrm{Hu}(2013)$ proposed two different models with different assumptions and also compared their model with the model of Bianco (2009). The models included the cross immunity class. At this class, the individuals are partially temporarily immune and the constant rate $\phi$ describes the ADE factor. The differences among Bianco (2009) model and the others two models 
are that $\mathrm{Hu}$ (2013) proposed the model with vector population and included the exposure class for human population. Numerical simulation and sensitivity analysis were made in order to plot a Hopf bifurcation which occurs in both models.

Aguiar (2007) also proposed an Ordinary Differential Equations (ODE) system for two serotypes of Dengue virus. Also, the total population was divided in classes which the individuals are identified by its clinical disease states. The assumptions for the model were concerned to the total population which remains constant in time, therefore the birth and the death rates were constant and equal. And, as the other models summarized up to the present moment, $\phi$ is the parameter that describes the ADE factor. In this model $R_{i}$ describes the population class that represents the individuals who are temporarily immune to the other serotypes once infected by one serotype. Time series simulations were investigated and various bifurcation phenomena were observed.

Theoretical mathematical analysis of the Aguiar (2007) model was made by Aguiar (2008) and by Kooi (2014). Aguiar (2008) has quantified the attractor structure, limit cycle and chaotic attractor by calculating Lyapunov exponents as a consequence from her previously paper. Analytic formulations for the equilibrium and analysis of the bifurcation structure were obtained for the symmetric case (when the strains are the same).

Kooi (2014) used the same model proposed by Aguiar (2007) to describe some numerical and analytical results. As a consequence of the works done by Aguiar (2008), Kooi (2014) made some numerical analysis to observe the dynamical behaviour of the model. Furthermore, they described the analytic steady states and the stability for the Disease Free equilibrium and for the boundary equilibrium, used on the numerical simulation to obtain the bifurcations.

These models aforementioned were formulated in terms of ordinary differential equations, without incorporating time lags. Therefore, there is no time delay in any state class for human population, neither for recovery nor incubation time. They consider that the recovery time acts instantaneously.

Even though these models consider the recovery time acts instantaneously, it is very important to assume time delay as a mechanism to model the dynamic of Dengue fever, because the immune response acts temporarily, protecting the body from second infections (Ferguson, 1999a; Hu, 2013; Guzman, 2013; Reich, 2013).

Simpler delay models are being used a lot in applications to describe biological models and infectious diseases dynamics. Delay Differential Equations (DDE) are useful on attempt to describe, for instance, the population or disease behaviour which intrinsically depends on 
a certain period of past time. Delay models were used in epidemiology by Cooke (1999) and Hethcote (1981) in order to determine when the time delay affects in periodc oscillations, as well as in models described by Hethcote (1989).

A SIR (susceptible, infected, recovered) model described by Kermack e McKendrick assumes that the recovered period is exponentially distributed and this assumption results in, obviously, an ODE system (Brauer, 2008).

Models with general time delay for recovery were used by Van den Driessche (1996). In this work, Van den Driessche (1996) used the special case of step function for the recovered period. Models with time delay in infected class were analysed by Cooke (1973) and on SIRS model (susceptible, infected, recovered, susceptible) with general time delay for recovery by Hethcote (1989).

Motivated by the spread of Malaria with focus and observation of the incubation time of the parasite, $\mathrm{Nah}$ (2014) has recently described a mathematical model for Malaria transmission with time delay on exposure class and with a general incubation period. Whereas, previously authors as Xiao (2013) used exponential function and step function in order to describe the incubation period, $\mathrm{Nah}$ (2014) assumed for the short term incubation period an exponential distribution, while for the long term incubation a fixed length, as a consequence, the distribution $P(t)$ is a weighted sum of exponential and a step function.

A mathematical model describing a general multi-strain disease has also been recently described by Chen (2016). The authors proposed a delay diffusive two strain disease model considering the SIR structure, constant recruitment rate and constant time delay representing the length of immunity period. In that work the stability of the model was determined by the Basic Reproduction Number.

A model for Dengue fever that describes a time delay between infectious and infected host was described by Sakdanupahp (2009). This model is relatively simple, with four equations, but brings two different constant time delays, one for infectious host to infected host and, the other, for vectors population. The study included vector population, but does not model different serotypes. Steady states were obtained, stability was proved and numerical analysis and comparison data were made.

As well as the study presented by Chen (2016), the model described by Cai (2013) preserves the SIR structure, models multi-strains, includes incubation period time, nevertheless in the model of the Cai (2013) there is an equation describing the vector population dynamic. In the paper, the authors were focused on the evolution of parasite or virus, once according to Cai 
(2013) the model could describe both: the dynamics of Dengue fever or Malaria. Also, an interval time for maturation was added to the model and this distributed delay was placed in order to describe the incubation period for the virus (or parasite). Moreover, the Basic Reproduction Number was obtained by biological definition.

Guan (2017) also described a Dengue fever model with time delay. The time delay included in the model refers to a time incubation of the virus in an infected population and in an infected mosquito population. In this work, numerical simulations and analytical results were obtained taking four cases for the constant time delay. They proposed a model containing only one serotype, however it was added the dynamics of vector population.

Models including distributed delay were recently used to described different kinds of epidemics. For instance, a SVEIR model with imperfect vaccination was formulated by Wang (2016b) to study the impact of vaccination on the mumps transmission. The distributed delay was used to describe a general latent distribution in the latent population class. Constructing Lyapunov functional, the authors have showed global stability of the model and discussed whether the disease will die out or becomes endemic depending on the size of the Vaccination Reproduction number.

Taking into account a simpler SIR model, Huang (2016) considered an infinite distributed delay on complex population network in order to deliver some on insights biological and social networks. Numerical experiments confirm that, in this case, the delay slows down the extinction of the disease when the threshold value is smaller than 1 , while the delay accelerates the spreading if it is bigger than 1.

Distributed delay was also used by $\mathrm{Xu}(2017)$ in a SVEIR model with vaccination and general incidence function. Based on a SVEIR model with continuous vaccination strategy, $\mathrm{Xu}$ (2017) improved the model considering heterogeneity to understand the effects of infinity distributed time delay and vaccination in the transmission of infection diseases. Results showed that distributed delay has no impact on the qualitative behaviour and global dynamics.

\subsection{Model Formulation and Application}

In this section, based on the theoretical works that study epidemics disease aforementioned, we are going to propose a model, namely, a system of integro-differential equation to describe a multi-strain disease, motivated by Dengue fever.

Let $N(t)$ be the total population of individuals at time $t$ in a region. We divided the 
population in disjoint classes according to the individual condition (status): susceptible for all serotypes, infected by serotype $i$, temporarily immune for all serotypes after being infected by serotype $i$, recovered for serotype $i$ but susceptible to the others and, recovered for all serotypes, represented at time $t$, respectively by $S(t), I_{i}(t), C_{i}(t), R_{i}(t)$ e $R(t)$. Furthermore, we include two more classes for the population, $I_{i j}(t)$, representing the sub-population reinfected by serotype $j$ after being infected by serotype $i$, with $i, j=1,2, i \neq j$.

Considering $r$ the growth rate and $k$ the carrying capacity, we assume the Ricker function $\left(F(N)=r N(t) e^{-k N(t)}\right)$ for birth in the susceptible class and $d$ a constant natural mortality rate for human population. We assume that the constant rate $\beta_{i}$ is the average number of effective contacts per infected individual, per unit time, by serotype $i$. Whereas the constant rate $\alpha_{j}$ is the probability of being infected again by different serotype, by serotype $j$.

It is important to note that the mosquito population is not considered explicitly in the model. Consequently, the rate $\beta_{i}$ and $\alpha_{j}$ will be the average number of bite and, the probability of a susceptible individual being bite for an infected mosquito by serotype $i$ and, the probability of a recovered individual by serotype $i$ being bite again for an infected mosquito by serotype $j$, respectively. This way, $\beta_{i}=b \frac{q_{i}}{V} b_{i}$, and $\alpha_{j}=b \frac{q_{j}}{V} a_{j}$, where $b$ is the average number of bite per mosquito per human, $q_{i}$ is the number of infected mosquitoes by serotype $i, b_{i}$ the probability of a susceptible individual being bitten for an infected mosquito by serotype $i$, and $a_{j}$ the probability of a recovered individual by serotype $i$ being bitten again for an infected mosquito by serotype $j$ and, $V$ represents the total population of mosquitoes.

Individuals in the infectious classes, $I_{i}(t)$, remain in this class with average time $\frac{1}{\gamma}$, since we assume that the length in this class is exponentially distributed. Once in infected class, the individual recovers and goes to the temporarily immune class $C_{i}(t)$. In this class, the individual gets temporary immunity for all serotypes. After that, the individual becomes susceptible again to the other serotypes and permanently recovered to the one that became infected.

Moreover, we assume for $C_{i}$ class a more general length of immunity. Let $P^{i}(t)$ be the function that describes the immunity period, the time that an individual remains in the $C_{i}$ class, $t$ units time after getting in this class. It is reasonable to assume that

$$
P^{i}(0)=1 \text { and } \quad P^{i}(\infty)=0,
$$


and, satisfies that $P^{i}(t)$ is non-increasing and

$$
\int_{0}^{\infty} P^{i}(s) d s=\frac{1}{\omega_{i}}<\infty .
$$

Once in temporarily immune class, the individual recovers and gets long life immunity to that serotype, even though it is still susceptible for the other serotypes. Hence, an individual in the $R_{i}$ class can be infected again with a probability $\alpha_{j}$, described previously. Only after being infected by all dengue serotypes the individual becomes immune and recovers at a constant rate $\gamma$ and remains life long recovered.

Whenever the individual is infected again by a different serotype, an increase risk of developing complications and aggravation for the disease happens. The pre-existence of dengue virus antibodies is a significant risk factor for severe disease such as the Dengue hemorrhagic fever (DHF) (Guzman, 2013; Gubler, 2014). This happens because the immune system responds to a different, but very similar serotype, increasing the infectiousness and enhancing viral replication (Gubler, 2014, Hu, 2013). This effect is called Antibody-Dependent Enhancement (ADE), when secondary infections are possible in the presence of low level of antibody, which causes growth of the viral replication (Gubler, 2014, Guzman, 2013).

According to Ferguson (1999a), this viral growth is associated with the transmissibility of the disease, therefore reinfected individuals are more infectious than during the first infection. In this model, the epidemiological consequence ADE will be described through the constant coefficient $\phi$, which represents the degree of enhancement, where it is assumed that previously exposure to one serotype causes the increase of the reinfection, therefore, an increasing of the susceptibility for reinfection.

Thus, not considering possible deaths caused by disease, the model can be summarized on the follow diagram: 


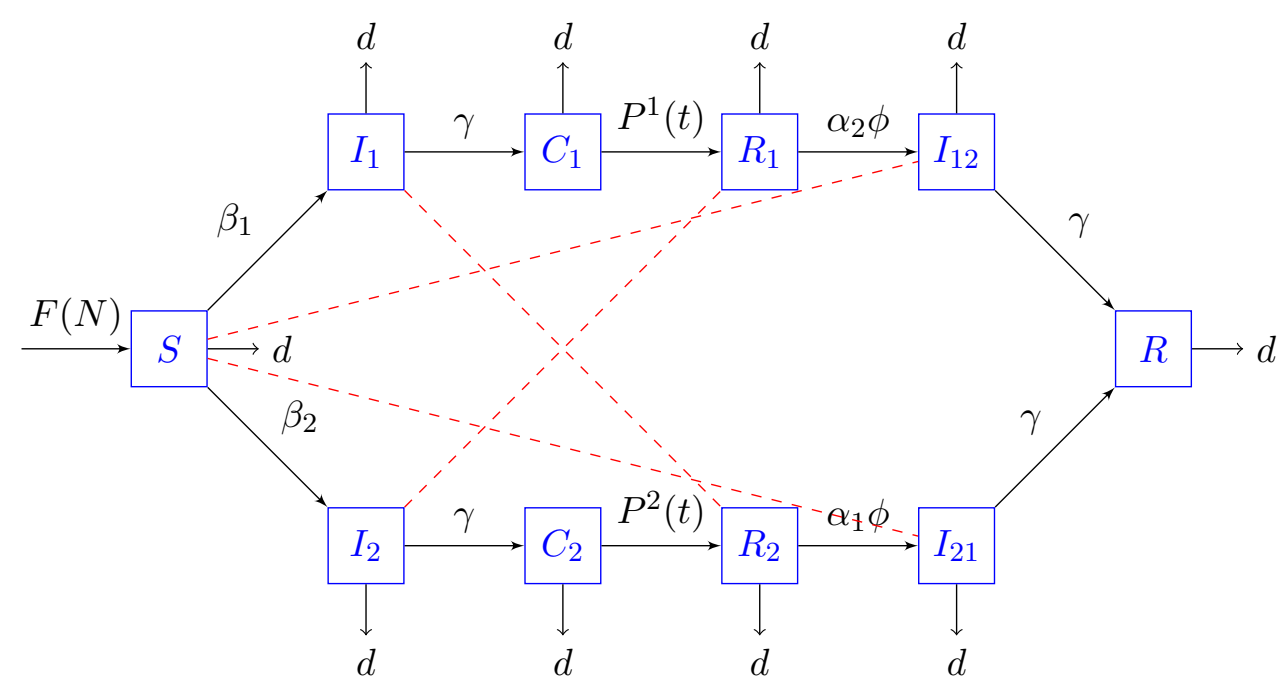

Figure 1.1 Flowchart for the compartmental model. Each frame refers to a compartment in which individual can reside according to its clinical disease state and, the arrows indicate the direction of movement of individuals among classes.

Based on works by Hethcote (1989), Wang (2016a), Cooke (1996), Van den Driessche (2007b) and in the previous assumptions, the model uses that the force of infection is given by standard incidence. The standard incidence is chosen, generally, for diseases which contact cannot increase indefinitely and it is limited even if the population size increases (Martcheva, 2015). In addition, a small number of infected is initially introduced into a region where population is susceptible, $S(0)>0, I_{i}(0)>0, C_{i}(0)=0, I_{21}(0)=0, I_{12}(0)=0, R_{i}(0)=0$ and $R(0)=0$. Therefore, the model can be described as a system of Integro-Differential Equations (IDE) as follows: 


$$
\begin{aligned}
\frac{d S(t)}{d t} & =r N(t) e^{-k N(t)}-d S(t)-\beta_{1} \frac{S(t)}{N(t)} I_{1}(t)-\beta_{2} \frac{S(t)}{N(t)} I_{2}(t)-\beta_{2} \frac{S(t)}{N(t)} I_{12}(t)-\beta_{1} \frac{S(t)}{N(t)} I_{21}(t) \\
\frac{d I_{1}(t)}{d t} & =-d I_{1}(t)+\beta_{1} \frac{S(t)}{N(t)} I_{1}(t)+\beta_{1} \frac{S(t)}{N(t)} I_{21}(t)-\gamma I_{1}(t) \\
\frac{d I_{2}(t)}{d t} & =-d I_{2}(t)+\beta_{2} \frac{S(t)}{N(t)} I_{2}(t)+\beta_{2} \frac{S(t)}{N(t)} I_{12}(t)-\gamma I_{2}(t) \\
C_{1}(t) & =\int_{0}^{t} \gamma I_{1}(s) P^{1}(t-s) e^{-d(t-s)} d s \\
C_{2}(t) & =\int_{0}^{t} \gamma I_{1}(s) P^{2}(t-s) e^{-d(t-s)} d s \\
\frac{d R_{1}(t)}{d t} & =-d R_{1}(t)-\alpha_{2} \phi \frac{R_{1}(t)}{N(t)} I_{12}(t)-\alpha_{2} \phi \frac{R_{1}(t)}{N(t)} I_{2}(t)-\int_{0}^{t} \gamma I_{1}(s) P_{t}^{1}(t-s) e^{-d(t-s)} d s \\
\frac{d R_{2}(t)}{d t} & =-d R_{2}(t)-\alpha_{1} \phi \frac{R_{2}(t)}{N(t)} I_{21}(t)-\alpha_{1} \phi \frac{R_{2}(t)}{N(t)} I_{1}(t)-\int_{0}^{t} \gamma I_{2}(s) P_{t}^{2}(t-s) e^{-d(t-s)} d s \\
\frac{d I_{12}(t)}{d t} & =-d I_{12}(t)-\gamma I_{12}(t)+\alpha_{2} \phi \frac{R_{1}(t)}{N(t)} I_{2}(t)+\alpha_{2} \phi \frac{R_{1}(t)}{N(t)} I_{12}(t) \\
\frac{d I_{21}(t)}{d t} & =-d I_{21}(t)-\gamma I_{21}(t)+\alpha_{1} \phi \frac{R_{2}(t)}{N(t)} I_{1}(t)+\alpha_{1} \phi \frac{R_{2}(t)}{N(t)} I_{21}(t) \\
\frac{d R(t)}{d t} & =-d R(t)+\gamma I_{12}(t)+\gamma I_{21}(t)
\end{aligned}
$$

where $N(t)$ is the total population on time $t$ and the equation which describes the dynamics of the total population is given by

$$
\frac{d N(t)}{d t}=r N(t) e^{-k N(t)}-d N(t)
$$

Note that the population of temporarily immune individuals was expressed by the integral

$$
C_{i}(t)=\int_{0}^{t} \gamma I_{i}(s) P^{i}(t-s) e^{-d(t-s)} d s
$$

Then, differentiating (1.5) gives

$$
\frac{d C_{i}(t)}{d t}=\gamma I_{i}(t) P^{i}(0)-d C_{i}(t)+\int_{0}^{t} \gamma I_{i}(s) P_{t}^{i}(t-s) e^{-d(t-s)} d s
$$

where $P_{t}^{i}$ denotes the derivative of $P^{i}(t-s)$ with respect to $t$. Since $P^{i}(0)=1$, it give us the differential equation for $C_{i}$.

Thus, the IDE system can be described as follows: 


$$
\begin{aligned}
& \left.\frac{d S(t)}{d t}\right)=r N(t) e^{-k N(t)}-d S(t)-\beta_{1} \frac{S(t)}{N(t)} I_{1}(t)-\beta_{2} \frac{S(t)}{N(t)} I_{2}(t)-\beta_{2} \frac{S(t)}{N(t)} I_{12}(t)-\beta_{1} \frac{S(t)}{N(t)} I_{21}(t) \\
& \frac{d I_{1}(t)}{d t}=-d I_{1}(t)+\beta_{1} \frac{S(t)}{N(t)} I_{1}(t)+\beta_{1} \frac{S(t)}{N(t)} I_{21}(t)-\gamma I_{1}(t) \\
& \frac{d I_{2}(t)}{d t}=-d I_{2}(t)+\beta_{2} \frac{S(t)}{N(t)} I_{2}(t)+\beta_{2} \frac{S(t)}{N(t)} I_{12}(t)-\gamma I_{2}(t) \\
& \frac{d C_{1}(t)}{d t}=\gamma I_{1}(t)-d C_{1}(t)+\int_{0}^{t} \gamma I_{1}(s) P_{t}^{1}(t-s) e^{-d(t-s)} d s \\
& \frac{d C_{2}(t)}{d t}=\gamma I_{2}(t)-d C_{2}(t)+\int_{0}^{t} \gamma I_{2}(s) P_{t}^{2}(t-s) e^{-d(t-s)} d s \\
& \frac{d R_{1}(t)}{d t}=-d R_{1}(t)-\alpha_{2} \phi \frac{R_{1}(t)}{N(t)} I_{12}(t)-\alpha_{2} \phi \frac{R_{1}(t)}{N(t)} I_{2}(t)-\int_{0}^{t} \gamma I_{1}(s) P_{t}^{1}(t-s) e^{-d(t-s)} d s \\
& \frac{d R_{2}(t)}{d t}=-d R_{2}(t)-\alpha_{1} \phi \frac{R_{2}(t)}{N(t)} I_{21}(t)-\alpha_{1} \phi \frac{R_{2}(t)}{N(t)} I_{1}(t)-\int_{0}^{t} \gamma I_{2}(s) P_{t}^{2}(t-s) e^{-d(t-s)} d s \\
& \frac{d I_{12}(t)}{d t}=-d I_{12}(t)-\gamma I_{12}(t)+\alpha_{2} \phi \frac{R_{1}(t)}{N(t)} I_{2}(t)+\alpha_{2} \phi \frac{R_{1}(t)}{N(t)} I_{12}(t) \\
& \frac{d I_{21}(t)}{d t}=-d I_{21}(t)-\gamma I_{21}(t)+\alpha_{1} \phi \frac{R_{2}(t)}{N(t)} I_{1}(t)+\alpha_{1} \phi \frac{R_{2}(t)}{N(t)} I_{21}(t) \\
& \frac{d R(t)}{d t}=-d R(t)+\gamma I_{12}(t)+\gamma I_{21}(t) \\
& \frac{d N(t)}{d t}=r N(t) e^{-k N(t)}-d N(t) .
\end{aligned}
$$

It is important to note that if we assume the length of immunity being exponentially distributed, which means that the probability of temporarily immune individual remains in the $C_{i}$ class is $P^{i}(t)=e^{-\omega_{i} t}$, with $\omega_{i}>0$, for $i=1,2$, then the system of Integro-Differential Equations becomes a system of Ordinary Differential Equations. Of course, this exponential function satisfies the assumptions required for $P^{i}(t)$, then the ODE system can be described as follows: 


$$
\begin{aligned}
& \frac{d S(t)}{d t}=r N(t) e^{-k N(t)}-d S(t)-\beta_{1} \frac{S(t)}{N(t)} I_{1}(t)-\beta_{2} \frac{S(t)}{N(t)} I_{2}(t)-\beta_{2} \frac{S(t)}{N(t)} I_{12}(t)-\beta_{1} \frac{S(t)}{N(t)} I_{21}(t) \\
& \frac{d I_{1}(t)}{d t}=-d I_{1}(t)+\beta_{1} \frac{S(t)}{N(t)} I_{1}(t)+\beta_{1} \frac{S(t)}{N(t)} I_{21}(t)-\gamma I_{1}(t) \\
& \frac{d I_{2}(t)}{d t}=-d I_{2}(t)+\beta_{2} \frac{S(t)}{N(t)} I_{2}(t)+\beta_{2} \frac{S(t)}{N(t)} I_{12}(t)-\gamma I_{2}(t) \\
& \frac{d C_{1}(t)}{d t}=\gamma I_{1}(t)-d C_{1}(t)-\omega_{1} C_{1}(t) \\
& \frac{d C_{2}(t)}{d t}=\gamma I_{2}(t)-d C_{2}(t)-\omega_{2} C_{2}(t) \\
& \frac{d R_{1}(t)}{d t}=-d R_{1}(t)-\alpha_{2} \phi \frac{R_{1}(t)}{N(t)} I_{12}(t)-\alpha_{2} \phi \frac{R_{1}(t)}{N(t)} I_{2}(t)+\omega_{1} C_{1}(t) \\
& \frac{d R_{2}(t)}{d t}=-d R_{2}(t)-\alpha_{1} \phi \frac{R_{2}(t)}{N(t)} I_{21}(t)-\alpha_{1} \phi \frac{R_{2}(t)}{N(t)} I_{1}(t)+\omega_{2} C_{2}(t) \\
& \frac{d I_{12}(t)}{d t}=-d I_{12}(t)-\gamma I_{12}(t)+\alpha_{2} \phi \frac{R_{1}(t)}{N(t)} I_{2}(t)+\alpha_{2} \phi \frac{R_{1}(t)}{N(t)} I_{12}(t) \\
& \frac{d I_{21}(t)}{d t}=-d I_{21}(t)-\gamma I_{21}(t)+\alpha_{1} \phi \frac{R_{2}(t)}{N(t)} I_{1}(t)+\alpha_{1} \phi \frac{R_{2}(t)}{N(t)} I_{21}(t) \\
& \frac{d R(t)}{d t}=-d R(t)+\gamma I_{12}(t)+\gamma I_{21}(t) \\
& \frac{d N(t)}{d t}=r N(t) e^{-k N(t)}-d N(t) .
\end{aligned}
$$




\section{Analysis of the Time Delay System}

Over the previous chapter, we have proposed a model to study the spread of Dengue fever in individuals in a region. Over the present chapter, we are going to analyse the system which the cross-immunity protection time can be defined as any continuous function with some additional properties, already defined in second section.

\subsection{Limiting System}

We are going to analyse the model 1.7 assuming the length of immunity $P^{i}(t)$ being a general distribution satisfying the assumption 1.1 and $(1.2)$. Observe that the equation that describes the dynamic for individuals in the recovered class is decoupled, hence we can put

$$
R(t)=N(t)-S(t)-I_{1}(t)-I_{2}(t)-C_{1}(t)-C_{2}(t)-R_{1}(t)-R_{2}(t)-I_{12}(t)-I_{21}(t) .
$$

Also, the equation that describes the dynamics of total population in time does not depend on the individual disease state, in other words, the disease does not interfere on the dynamic of total population. Thus, searching for the steady state of the equation

$$
\frac{d N(t)}{d t}=r N(t) e^{-k N(t)}-d N(t)
$$


it is found a unique positive equilibrium

$$
N^{*}=\frac{1}{k} \ln \left(\frac{r}{d}\right), \quad r>d
$$

Therefore, assuming that the size of population $N(t)$ is constant in time, namely, the total population reaches the equilibrium $N^{*}$, we search for constants functions $S^{*}, I_{1}^{*}, I_{2}^{*}, C_{1}^{*}$, $C_{2}^{*}, R_{1}^{*}, R_{2}^{*}, I_{12}^{*}, I_{21}^{*}$, which satisfy:

$$
\begin{aligned}
& 0=N^{*} d-d S^{*}-\overline{\beta_{1}} S^{*}\left(I_{1}^{*}+I_{21}^{*}\right)-\overline{\beta_{2}} S^{*}\left(I_{2}^{*}+I_{12}^{*}\right) \\
& 0=-(d+\gamma) I_{1}^{*}+\overline{\beta_{1}} S^{*} I_{1}^{*}+\overline{\beta_{1}} S^{*} I_{21}^{*} \\
& 0=-(d+\gamma) I_{2}^{*}+\overline{\beta_{2}} S^{*} I_{2}^{*}+\overline{\beta_{2}} S^{*} I_{12}^{*} \\
& 0=\gamma I_{1}^{*}-d C_{1}^{*}+\gamma I_{1}^{*} \int_{0}^{t} P_{t}^{1}(t-s) e^{-d(t-s)} d s \\
& 0=\gamma I_{2}^{*}-d C_{2}^{*}+\gamma I_{2}^{*} \int_{0}^{t} P_{t}^{2}(t-s) e^{-d(t-s)} d s \\
& 0=-d R_{1}^{*}-\overline{\alpha_{2}} \phi R_{1}^{*}\left(I_{12}^{*}+I_{2}^{*}\right)-\gamma I_{1}^{*} \int_{0}^{t} P_{t}^{1}(t-s) e^{-d(t-s)} d s \\
& 0=-d R_{2}^{*}-\overline{\alpha_{1}} \phi R_{2}^{*}\left(I_{21}^{*}+I_{1}^{*}\right)-\gamma I_{2}^{*} \int_{0}^{t} P_{t}^{2}(t-s) e^{-d(t-s)} d s \\
& 0=-(d+\gamma) I_{12}^{*}+\overline{\alpha_{2}} \phi R_{1}^{*}\left(I_{2}^{*}+I_{12}^{*}\right) \\
& 0=-(d+\gamma) I_{21}^{*}+\overline{\alpha_{1}} \phi R_{2}^{*}\left(I_{1}^{*}+I_{21}^{*}\right)
\end{aligned}
$$

where $\overline{\beta_{i}}=\frac{\beta_{i}}{N^{*}}$ and $\overline{\alpha_{i}}=\frac{\alpha_{i}}{N^{*}}$.

Clearly, the system (1.7) always has a disease-free equilibrium, namely,

$$
D_{0}=\left(N^{*}, 0,0,0,0,0,0,0,0,0\right)
$$

and, $R^{*}=N^{*}-S^{*}-I_{1}^{*}-I_{2}^{*}-C_{1}^{*}-C_{2}^{*}-R_{1}^{*}-R_{2}^{*}-I_{12}^{*}-I_{21}^{*}$.

For the purpose of analysis of the dynamic behaviour and stability of the zero solution of the delay system (1.7), we must note that we are working with an IDE system consisting of Volterra Integro-differential Equations of convolution type of the form:

$$
\frac{d x(t)}{d t}=f(t, x)+\int_{0}^{t} h(t-s) g(s, x(s)) d s, \quad x(0)=\xi,
$$

where $h$ is called kernel function of the equation.

According to Miller (1972), if kernel function is integrable, the asymptotic stability of the zero solution of the linear system will be known just by looking if there are no solutions in 
the right half of plane $\mathcal{R} e z \geq 0$ of $\operatorname{det}(z I-A-\hat{H})=0$, where $A$ is the linear part of the system and $\hat{H}$ denotes the Laplace transform of the linear part of $h$.

In Miller (1972), Miller (1971) and Brauer (1978) a whole theory can be found about the Volterra Integral equations, as well as the Volterra Integro-differential equations, and those will be useful for our purpose here. Also, Feng (2016) and Hethcote (1981) used the same approach to demonstrate the results of stability theorems. According to Hethcote (1981), using Miller's theorems, it is possible to show that the general problem is well posed and the equilibrium of the system corresponds with those of their limiting equation. Also, according to Miller (1971), the limiting system ensures that the initial system proposed involving non-linear Volterra Integro-differential equations has an equilibrium.

Following the idea by Brauer (1978), we consider the following limiting system for the initial system (1.7):

$$
\begin{aligned}
& \frac{d S(t)}{d t}=d N^{*}-d S(t)-\overline{\beta_{1}} S(t)\left(I_{1}(t)+I_{21}(t)\right)-\overline{\beta_{2}} S(t)\left(I_{2}(t)+I_{12}(t)\right) \\
& \frac{d I_{1}(t)}{d t}=-(d+\gamma) I_{1}(t)+\overline{\beta_{1}} S(t)\left(I_{1}(t)+I_{21}(t)\right) \\
& \frac{d I_{2}(t)}{d t}=-(d+\gamma) I_{2}(t)+\overline{\beta_{2}} S(t)\left(I_{2}(t)+I_{12}(t)\right) \\
& \frac{d C_{1}(t)}{d t}=\gamma I_{1}(t)-d C_{1}(t)+\int_{0}^{\infty} \gamma I_{1}(s) P_{t}^{1}(t-s) e^{-d(t-s)} d s \\
& \frac{d C_{2}(t)}{d t}=\gamma I_{2}(t)-d C_{2}(t)+\int_{0}^{\infty} \gamma I_{2}(s) P_{t}^{2}(t-s) e^{-d(t-s)} d s \\
& \frac{d R_{1}(t)}{d t}=-d R_{1}(t)-\overline{\alpha_{2}} \phi R_{1}(t)\left(I_{12}(t)+I_{2}(t)\right)-\int_{0}^{\infty} \gamma I_{1}(s) P_{t}^{1}(t-s) e^{-d(t-s)} d s \\
& \frac{d R_{2}(t)}{d t}=-d R_{2}(t)-\overline{\alpha_{1}} \phi R_{2}(t)\left(I_{21}(t)+I_{1}(t)\right)-\int_{0}^{\infty} \gamma I_{2}(s) P_{t}^{2}(t-s) e^{-d(t-s)} d s \\
& \frac{d I_{12}(t)}{d t}=-(d+\gamma) I_{12}(t)+\overline{\alpha_{2}} \phi R_{1}(t)\left(I_{2}(t)+I_{12}(t)\right) \\
& \frac{d I_{21}(t)}{d t}=-(d+\gamma) I_{21}(t)+\overline{\alpha_{1}} \phi R_{2}(t)\left(I_{1}(t)+I_{21}(t)\right) \\
& \frac{d R(t)}{d t}=-d R(t)+\gamma\left(I_{12}(t)+I_{21}(t)\right) .
\end{aligned}
$$

For this limiting system (2.4), it is necessary to define the Banach space with memory, as Feng (2016), Wang (2012), Li (2010) and Röst (2008) have done in order to have a wellposed system and solutions defined in $(-\infty, 0]$, since we have terms with infinite delay. We can choose the space of bounded continuous functions on $(-\infty, 0]$, however for qualitative theory will be useful to consider another space phase. 
Let $\Delta_{1}, \Delta_{2}$ be positive constants, such that $\Delta_{i}<d$, satisfying, for $i=1,2$,

$$
\int_{0}^{\infty} P_{t}^{i}(u) e^{-d u} e^{\Delta_{i} u} d u<\infty
$$

Define the Banach space, for $i=1,2$,

$$
X_{\Delta_{i}}=\left\{\Psi \in \mathcal{C}((-\infty, 0], \mathbb{R}): \Psi(s) e^{\Delta_{i} s} \text { is uniformly continuous in }(-\infty, 0] \text { and }\|\Psi\|_{e}<\infty\right\}
$$

where $\|\Psi\|_{e}=\sup _{s \leq 0}|\Psi(s)| e^{\Delta_{i} s}$. We consider $X=\mathbb{R} \times X_{\Delta_{1}} \times X_{\Delta_{2}} \times \mathbb{R}^{7}$ as the phase space for the limiting system (2.4).

Denote $I_{i_{t}}$, for $i=1,2$, the solution $I_{i}(t)$ at time $t$, which is $I_{i_{t}}(s)=I_{i}(t+s)$, $s \leq 0$. We have interest in non-negative solutions, which corresponds to non-negative functions of $X_{\Delta_{1}}$ and $X_{\Delta_{2}}$, for this reason, functions in $\Lambda_{i}=\left\{\Psi \in X_{\Delta_{i}}: \Psi(s) \geq 0, s \in(-\infty, 0]\right\}$, $i=1,2$.

Thus, for initial conditions, $S(0)=s_{0} \in \mathbb{R}_{+}, \quad I_{1_{0}}=\Psi_{1} \in \Lambda_{1}, \quad I_{2_{0}}=\Psi_{2} \in \Lambda_{2}$, $C_{i}(0)=c_{i} \in \mathbb{R}_{+}, R_{i}(0)=r_{i} \in \mathbb{R}_{+}, I_{j i}(0)=\theta_{i} \in \mathbb{R}_{+}, i=1,2$, the solutions of the limiting systems in $X$ remain non-negative and, $I_{i_{t}} \in X_{\Delta_{i}}$, for all $t$, for $i=1,2$.

Moreover, the following set $\Omega_{X}=\left\{\left(S, I_{1}(),. I_{2}(),. C_{1}, C_{2}, R_{1}, R_{2}, I_{12}, I_{21}, R\right) \in \mathbb{R}_{+} \times\right.$ $\left.\Lambda_{1} \times \Lambda_{2} \times \mathbb{R}_{+}^{7}: S+I_{1}(0)+I_{2}(0)+C_{1}+C_{2}+R_{1}+R_{2}+I_{12}+I_{21}+R \leq N^{*}\right\}$ is positively invariant for system (2.4).

In fact, by standard theory of functional differential equations, it is possible to verify that solutions of the system 2.4 with non-negative initial condition and $I_{i}(0) \geq 0$, has solutions $\left(S(t), I_{1_{t}}, I_{2_{t}}, C_{1}(t), C_{2}(t), R_{1}(t), R_{2}(t), I_{12}(t), I_{21}(t), R(t)\right)$ which remain non-negative for all $t \geq 0$.

Also, adding the equations of the system we have

$$
\begin{aligned}
& \frac{d S}{d t}+\frac{d I_{1_{t}}(0)}{d t}+\frac{d I_{2_{t}}(0)}{d t}+\frac{d C_{1}}{d t}+\frac{d C_{2}}{d t}+\frac{d R_{1}}{d t}+\frac{d R_{2}}{d t}+\frac{d I_{12}}{d t}+\frac{d I_{21}}{d t}+\frac{d R}{d t}= \\
& d N^{*}-d\left(S(t)+I_{1_{t}}(0)+I_{2_{t}}(0)+C_{1}(t)+C_{2}(t)+R_{1}(t)+R_{2}(t)+I_{12}(t)+I_{21}(t)+R(t)\right) .
\end{aligned}
$$

Hence

$$
\limsup _{t \rightarrow \infty}\left(S(t)+I_{1_{t}}(0)+I_{2_{t}}(0)+C_{1}(t)+C_{2}(t)+R_{1}(t)+R_{2}(t)+I_{12}(t)+I_{21}(t)+R(t)\right) \leq N^{*}
$$

This shows that solutions with initial conditions in $\mathbb{R}_{+} \times \Lambda_{1} \times \Lambda_{2} \times \mathbb{R}_{+}^{7}$ remain in $\Omega_{X}$ for all $t \geq 0$. 
The trivial equilibrium $D_{0}=\left(N^{*}, 0,0,0,0,0,0,0,0,0\right)$ is in the invariant set $\Omega_{X}$. Now, in order to find the other equilibriums of the limiting system (2.4), in the invariant set $\Omega_{X}$, will be necessary to rewrite the term with the limiting expression as well as we will need the following assumptions

$$
\int_{0}^{\infty} P^{i}(s) e^{-d s} d s<\infty \quad \text { and, } \quad \int_{0}^{\infty} s P^{i}(s) e^{-d s} d s<\infty
$$

Changing parameters in the expression we have, for $i=1,2$,

$$
h_{i}(t)=:-\int_{0}^{t} P_{t}^{i}(t-s) e^{-d(t-s)} d s=-\int_{0}^{t} P_{s}^{i}(s) e^{-d s} d s .
$$

Also, we have

$$
h_{i}(t)=-\int_{0}^{t} P_{s}^{i}(s) e^{-d s} d s=-\left[P^{i}(t) e^{-d t}-P(0)+d \int_{0}^{t} P^{i}(s) e^{-d s} d s\right] .
$$

Since by assumption (2.6), the average time that an individual remains temporarily immune before becoming susceptible again or die can be describe by

$$
\int_{0}^{\infty} P^{i}(s) e^{-d s} d s:=N_{i}
$$

where $0<N_{i}<1 / d$, and $N_{i}$ is constant, we have

$$
\begin{aligned}
h_{i}(\infty) & =-\int_{0}^{\infty} P_{t}^{i}(t-s) e^{-d(t-s)} d s \\
& =\lim _{t \rightarrow \infty}-\int_{0}^{t} P_{s}^{i}(s) e^{-d s} d s=1-d N_{i}:=M_{i} .
\end{aligned}
$$

Also the second assumption in (2.6) leads to a

$$
\int_{0}^{\infty} s P_{s}^{i}(s) e^{-d s} d s<\infty
$$

This result 2.9) will be necessary in order to use the stability theorems found in Miller (1972). It is obtained by integrating by parts the left side of the expression above and using the assumptions in (2.6).

Then, the equilibriums of the limiting system (2.4) in $\Omega_{X}$ are the same as those of the 
following system, in $\Omega$,

$$
\begin{aligned}
\frac{d S}{d t} & =d N^{*}-d S-\overline{\beta_{1}} S\left(I_{1}+I_{21}\right)-\overline{\beta_{2}} S\left(I_{2}+I_{12}\right) \\
\frac{d I_{1}}{d t} & =-(d+\gamma) I_{1}+\overline{\beta_{1}} S\left(I_{1}+I_{21}\right) \\
\frac{d I_{2}}{d t} & =-(d+\gamma) I_{2}+\overline{\beta_{2}} S\left(I_{2}+I_{12}\right) \\
\frac{d C_{1}}{d t} & =\gamma I_{1}-d C_{1}-\gamma I_{1} M_{1} \\
\frac{d C_{2}}{d t} & =\gamma I_{2}-d C_{2}-\gamma I_{2} M_{2} \\
\frac{d R_{1}}{d t} & =-d R_{1}-\overline{\alpha_{2}} \phi R_{1}\left(I_{12}+I_{2}\right)+\gamma I_{1} M_{1} \\
\frac{d R_{2}}{d t} & =-d R_{2}-\overline{\alpha_{1}} \phi R_{2}\left(I_{21}+I_{1}\right)+\gamma I_{2} M_{2} \\
\frac{d I_{12}}{d t} & =-(d+\gamma) I_{12}+\overline{\alpha_{2}} \phi R_{1}\left(I_{2}+I_{12}\right) \\
\frac{d I_{21}}{d t} & =-(d+\gamma) I_{21}+\overline{\alpha_{1}} \phi R_{2}\left(I_{1}+I_{21}\right)
\end{aligned}
$$

and, $R(t)=N^{*}-S(t)-I_{1}(t)-I_{2}(t)-C_{1}(t)-C_{2}(t)-R_{1}(t)-R_{2}(t)-I_{12}(t)-I_{21}(t)$.

Since $M_{i}$ is positive and smaller than one, in the case of the extinction of one of the strains, the boundary equilibriums of the system (2.4) are

$$
D_{1}=\left(\frac{d+\gamma}{\overline{\beta_{1}}}, \frac{d}{\overline{\beta_{1}}}\left[\frac{\overline{\beta_{1}} N^{*}}{d+\gamma}-1\right], 0, \frac{\gamma}{d}\left(1-M_{1}\right) I_{1}^{*}, 0, M_{1} \frac{\gamma}{\overline{\beta_{1}}}\left[\frac{\overline{\beta_{1}} N^{*}}{d+\gamma}-1\right], 0,0,0,0\right)
$$

and,

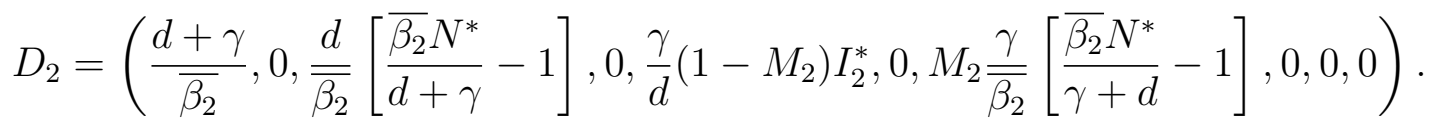

The boundary equilibriums, $D_{1}$ and $D_{2}$, will be in the $\Omega$ positively invariant region, as long as the parameters satisfy $\frac{N^{*} \overline{\beta_{1}}}{d+\gamma}>1$ and, $\frac{N^{*} \overline{\beta_{2}}}{d+\gamma}>1$, respectively.

In the case of coexistence of the two infection forces, we are able to find the equilib- 
rium, which is given by

$$
\begin{aligned}
C_{1}^{*} & =\frac{\gamma\left(1-M_{1}\right)}{d} I_{1}^{*} \\
C_{2}^{*} & =\frac{\gamma\left(1-M_{2}\right)}{d} I_{2}^{*} \\
R_{1}^{*} & =\frac{d+\gamma-\overline{\beta_{2}} S^{*}}{\overline{\alpha_{2}} \phi} \\
R_{2}^{*} & =\frac{d+\gamma-\overline{\beta_{1}} S^{*}}{\overline{\alpha_{1}} \phi} \\
I_{12}^{*} & =\frac{(d+\gamma) I_{2}^{*}-\overline{\beta_{2}} S^{*} I_{2}^{*}}{\overline{\beta_{2}} S^{*}} \\
I_{21}^{*} & =\frac{(d+\gamma) I_{1}^{*}-\overline{\beta_{1}} S^{*} I_{1}^{*}}{\overline{\beta_{1}} S^{*}} \\
I_{1}^{*}+I_{2}^{*} & =\frac{d\left(N^{*}-\overline{S^{*}}\right)}{d+\gamma}
\end{aligned}
$$

and, $S^{*}$ is the root of the cubic polynomial $O(S)=b_{3} S^{3}+b_{2} S^{2}+b_{1} S+b_{0}$ where

$$
\begin{aligned}
b_{3} & =\overline{\beta_{1} \beta_{2}}\left[\overline{\alpha_{2}}\left(d+\gamma\left(1-M_{1}\right)\right)\left(\overline{\beta_{1}}-\overline{\alpha_{1}} \phi\right)(d+\gamma)+\overline{\alpha_{1}}\left(d+\gamma\left(1-M_{2}\right)\right)\left(\overline{\beta_{2}}(d+\gamma)-\overline{\alpha_{2}} \phi \gamma M_{1}\right)\right] \\
b_{2} & =\overline{\alpha_{2}} \overline{\beta_{2}}(d+\gamma)\left(d+\gamma\left(1-M_{1}\right)\right)\left((d+\gamma)\left(\overline{\alpha_{1}} \phi-\overline{\beta_{1}}\right)+N^{*} \overline{\beta_{1}} \overline{\alpha_{1}} \phi\right) \\
& -\overline{\beta_{1}} \overline{\alpha_{2}}(d+\gamma)^{3}\left(\overline{\beta_{1}}-\overline{\alpha_{1}} \phi\right)+\overline{\beta_{2}} \overline{\alpha_{1}}(d+\gamma)^{2}\left(\overline{\alpha_{2}} \phi \gamma M_{1}-\overline{\beta_{2}}(d+\gamma)\right) \\
& -\overline{\beta_{2} \beta_{1}} \overline{\alpha_{1}}\left(d+\gamma\left(1-M_{2}\right)\right)\left((d+\gamma)^{2}-\overline{\alpha_{2}} \phi \gamma M_{1} N^{*}\right) \\
b_{1} & =(d+\gamma)^{3}\left[(d+\gamma)\left(\overline{\beta_{2}} \overline{\alpha_{1}}+\overline{\beta_{1}} \overline{\alpha_{2}}-\overline{\alpha_{2} \alpha_{1}} \phi\right)-N^{*} \overline{\alpha_{1} \alpha_{2}} \phi\left(\overline{\beta_{1}}+\overline{\beta_{2}}\right)\right] \\
b_{0} & =N^{*} \phi \overline{\alpha_{1} \alpha_{2}}(d+\gamma)^{4} .
\end{aligned}
$$

Moreover, $S^{*}$ has to satisfy that $S^{*}<\frac{d+\gamma}{\overline{\beta_{i}}}, i=1,2$, in order to have the equilibrium in the $\Omega$ region. Otherwise, if $S^{*}$ does not satisfy the inequality, the variables which represent recovered and infected populations will be negative.

Now, we are ready to prove the theorems about the existence of the equilibriums of the system (2.4). Yet, we need to define an important epidemiological threshold before, which is going to help us to prove the existence theorems as well as giving us the stability of these equilibriums.

\subsubsection{Basic Reproduction Number}

The Basic Reproduction Number $\mathcal{R}_{0}$ is defined by many authors, such as Van den Driessche (2008), as the expected number of secondary infections produced by one case in a 
susceptible population, also as a measure of the potential for disease spread in a population. Mathematically, the Basic Reproduction Number is a threshold for stability of Disease Free equilibrium (Van den Driessche, 2008).

Thereby, we define the threshold value

$$
\mathcal{R}_{0}=\frac{N^{*} \beta}{d+\gamma}
$$

as the Basic Reproduction Number of the system for symmetric case, when the parameters are equal. Also, the threshold values

$$
\mathcal{R}_{1}=\frac{N^{*} \overline{\beta_{1}}}{d+\gamma} \quad \text { and } \quad \mathcal{R}_{2}=\frac{N^{*} \overline{\beta_{2}}}{d+\gamma}
$$

as the Basic Reproduction Number, for asymmetric case, regarding to infection one and two, respectively.

In this case, it is usual to define an overall Reproduction Number for the system when it is a multi-strain model with different strains. Thus, the Basic Reproduction Number for the system, concerning to asymmetric case will be defined as:

$$
\mathcal{R}_{0}=\max \left\{\mathcal{R}_{1}, \mathcal{R}_{2}\right\}
$$

\subsubsection{Equilibriums of the Limiting System}

We are going to assume, without loss of generality, from now on that $\beta_{2} \geq \beta_{1}$. Therefore, either we have symmetry in the parameters, it means the infections have the same force, or one of the infections has the largest infection rate. In this case, $\mathcal{R}_{0}=\mathcal{R}_{2}$.

Theorem 1. If $\mathcal{R}_{1}>1$ then the system of equations (2.4), always has the boundary equilibrium, $D_{1}$, in $\Omega_{X}$, where

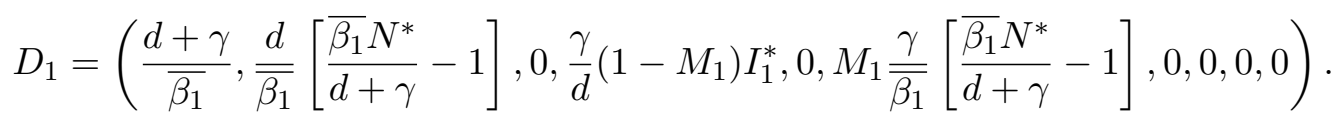

And, if $\mathcal{R}_{2}>1$ the system of equations (2.4) always has the boundary equilibrium, $D_{2}$, in $\Omega_{X}$, where

$$
D_{2}=\left(\frac{d+\gamma}{\overline{\beta_{2}}}, 0, \frac{d}{\overline{\beta_{2}}}\left[\frac{\overline{\beta_{2}} N^{*}}{d+\gamma}-1\right], 0, \frac{\gamma}{d}\left(1-M_{2}\right) I_{2}^{*}, 0, M_{2} \frac{\gamma}{\overline{\beta_{2}}}\left[\frac{\overline{\beta_{2}} N^{*}}{\gamma+d}-1\right], 0,0,0\right) .
$$


Proof. It is easy to see that, if $\frac{N^{*} \overline{\beta_{i}}}{d+\gamma}>1$, for $i=1,2$ then $I_{i}^{*}, C_{i}^{*}$ and $R_{i}^{*}$, for $i=1,2$ are positive and the total sum is $N^{*}$. Thus, $D_{i}$ is in $\Omega, i=1,2$ and the system has always a boundary equilibrium with that condition.

Theorem 2. If $\max \mathcal{R}_{0}>1$ and,

$$
R_{\text {Inv }}=\frac{\overline{\beta_{1}}}{\bar{\beta}_{2}}+\left(\frac{N^{*} \overline{\beta_{2}}}{d+\gamma}-1\right) \frac{\overline{\alpha_{1}} \phi \gamma M_{2}}{\overline{\beta_{2}}(d+\gamma)}>1
$$

then, the system (2.4) admits an equilibrium of the coexistence with the two strains in $\Omega_{X}$.

Proof. The independent term, $b_{0}$, of the polynomial $O(S)$ is always positive. Since the equilibrium is given by (2.11) with $S^{*}$ being a root of the polynomial $O$, this equilibrium will be in the region $\Omega$ if $S^{*}<\frac{d+\gamma}{\overline{\beta_{i}}}$, for $i=1,2$, we define

$$
S_{\min }=\min \left\{\frac{d+\gamma}{\overline{\beta_{1}}}, \frac{d+\gamma}{\overline{\beta_{2}}}\right\} \text {. }
$$

It means, if $\frac{N^{*} \overline{\beta_{2}}}{d+\gamma}>1$ and $\mathcal{R}_{\text {Inv }}>1$, we have

$$
O\left(S_{\text {min }}\right)=\frac{\left[(d+\gamma)^{2} \gamma M_{1} \overline{\alpha_{2}} \overline{\beta_{1}}\right]}{{\overline{\beta_{2}}}^{2}}\left[(d+\gamma)^{2}\left(\overline{\beta_{2}}-\overline{\beta_{1}}\right)+\gamma M_{2} \overline{\alpha_{1}} \phi\left((d+\gamma)-N^{*} \overline{\beta_{2}}\right)\right]<0 .
$$

This shows that we have a root $S^{*}$ of the polynomial $O$, such that, $0<S^{*}<S_{\min }$ for $i=1,2$. Therefore, for this $S^{*}$, the positive equilibrium $D_{3}$ with the variables satisfying (2.11) is $\Omega$. Moreover, this shows that we have a positive equilibrium of the system, in $\Omega_{X}$, with the coexistence of the two strains.

\subsubsection{Symmetric Case}

Once we restrict to the case $\overline{\beta_{1}}=\overline{\beta_{2}}=\beta, \overline{\alpha_{1}}=\overline{\alpha_{2}}=\alpha$ and $P^{1}(t)=P^{2}(t)$, which implies that $M_{1}=M_{2}=M$, we can write the cubic polynomial $O(S)=Q(S)(b S+a)$ where

$$
\begin{aligned}
& a=-\alpha \phi(d+\gamma)^{2} \\
& b=\alpha \phi \beta(d+\gamma(1-M))
\end{aligned}
$$

and, $Q(S)=a_{2} S^{2}+a_{1} S+a_{0}$ where

$$
\begin{aligned}
& a_{2}=\beta[(d+\gamma)(\alpha \phi-2 \beta)+\gamma M \alpha \phi] \\
& a_{1}=(d+\gamma)^{2}(2 \beta-\alpha \phi)-N^{*} \alpha \phi \beta(d+\gamma+\gamma M) \\
& a_{0}=N^{*} \alpha \phi(d+\gamma)^{2} .
\end{aligned}
$$


Since $S^{*}=\frac{-a}{b}=\frac{(d+\gamma)^{2}}{\beta(d+\gamma(1-M))}$ gives us a negative value for $I_{12}^{*}$ and $I_{21}^{*}$, the equilibrium will not be in the $\Omega$ region, we just need to look for the $Q(S)$ roots.

While searching for the roots, we must remember that we want a $S^{*}$, root of $Q(S)$ polynomial, which is positive and, $S^{*}<\frac{d+\gamma}{\beta}$, in order to have an equilibrium in the $\Omega$ region. All these information give us the following theorem.

Theorem 3. If $\mathcal{R}_{0}>1$ the system of equations (2.4) restrict to the case $\overline{\beta_{1}}=\overline{\beta_{2}}=\beta$, $\overline{\alpha_{1}}=\overline{\alpha_{2}}=\alpha$ and $M_{1}=M_{2}=M$ always have two boundary equilibriums in $\Omega_{X}$, namely,

$$
\begin{aligned}
& D_{1}=\left(\frac{d+\gamma}{\beta}, \frac{d}{\beta}\left[\frac{\beta N^{*}}{d+\gamma}-1\right], 0, \frac{\gamma}{d}(1-M) I_{1}^{*}, 0, M \frac{\gamma}{\beta}\left[\frac{\beta N^{*}}{d+\gamma}-1\right], 0,0,0,0\right), \\
& D_{2}=\left(\frac{d+\gamma}{\beta}, 0, \frac{d}{\beta}\left[\frac{\beta N^{*}}{d+\gamma}-1\right], 0, \frac{\gamma}{d}(1-M) I_{2}^{*}, 0, M \frac{\gamma}{\beta}\left[\frac{\beta N^{*}}{d+\gamma}-1\right], 0,0,0\right)
\end{aligned}
$$

and, a unique positive equilibrium in $\Omega_{X}$, with coexistence of the two strains, where

$$
S^{*}=\frac{-a_{1}}{2 a_{2}}-\frac{\sqrt{a_{1}^{2}-4 a_{0} a_{2}}}{2 a_{2}},
$$

(with $a_{i}$ being the coefficients of polynomial $Q(S)$ ) and, $I_{1}^{*}=I_{2}^{*}=\frac{d}{2(d+\gamma)}\left(N-S^{*}\right), C_{1}^{*}=C_{2}^{*}$, $R_{1}^{*}=R_{2}^{*}, \quad I_{12}^{*}=I_{21}^{*}$ satisfies (2.11).

Proof. If $\frac{N^{*} \beta}{d+\gamma}>1$ then, it is easy to see that $D_{i}$ is in $\Omega$, for $i=1,2$.

In addition, since the searched root $S^{*}$ needs to be smaller than $\frac{d+\gamma}{\beta}$, let $S_{\max }$ be

$$
S_{\max }=\frac{d+\gamma}{\beta}
$$

Then, the quadratic polynomial evaluated in $S_{\max }$ is

$$
Q\left(S_{\max }\right)=Q\left(\frac{d+\gamma}{\beta}\right)=(d+\gamma) \gamma M \alpha \phi\left(\frac{d+\gamma}{\beta}-N^{*}\right)<0,
$$

because $\frac{N^{*} \beta}{d+\gamma}>1$.

Besides, the independent term, $a_{0}$, of the polynomial $Q(S)$ is positive. This proves that we have a positive root satisfying $S^{*}<\frac{d+\gamma}{\beta}$, and it is given by

$$
\begin{aligned}
S^{*} & =\frac{(d+\gamma)^{2}(-2 \beta+\alpha \phi)+N^{*} \alpha \phi \beta(d+\gamma+\gamma M)}{2 \beta[(d+\gamma)(\alpha \phi-2 \beta)+\gamma M \alpha \phi]} \\
& -\frac{\sqrt{\left((d+\gamma)^{2}(2 \beta-\alpha \phi)+N^{*} \alpha \phi \beta(d+\gamma+\gamma M)\right)^{2}-8 N^{*} \alpha \phi \beta^{2} \gamma M(d+\gamma)^{2}}}{2 \beta[(d+\gamma)(\alpha \phi-2 \beta)+\gamma M \alpha \phi]}
\end{aligned}
$$


Therefore, the equilibrium $D_{3}=\left(S^{*}, I_{1}^{*}, I_{2}^{*}, C_{1}^{*}, C_{2}^{*}, R_{1}^{*}, R_{2}^{*}, I_{12}^{*}, I_{21}^{*}, R^{*}\right)$ is in $\Omega$, where $S^{*}$ is given by 2.17) and, from 2.11,

$$
\begin{aligned}
& I_{1}^{*}=I_{2}^{*}=\frac{d\left(N^{*}-S^{*}\right)}{2(d+\gamma)}, \\
& C_{1}^{*}=C_{2}^{*}=\frac{\gamma(1-M)}{d} I_{1}^{*}, \\
& R_{1}^{*}=R_{2}^{*}=\frac{d+\gamma-\beta S^{*}}{\alpha \phi}, \\
& I_{12}^{*}=I_{21}^{*}=\frac{(d+\gamma) I_{1}^{*}-\beta S^{*} I_{1}^{*}}{\beta S^{*}}, \\
& R^{*}=N^{*}-S^{*}-I_{1}^{*}-I_{2}^{*}-C_{1}^{*}-C_{2}^{*}-R_{1}^{*}-R_{1}^{*}-I_{12}^{*}-I_{21}^{*} .
\end{aligned}
$$

\subsection{Stability Analysis}

In the previous section, we have calculated the equilibriums of the limiting system in order to know the equilibriums of the delay system. Over this section, we are going to introduce results that connect the local stability of the limiting system with the local stability of the delay system. In the sequence, we are going to analyse the stability of the limiting system (2.4) in order to use this results to achieve the main goal which involves understanding the dynamic behaviour of the initial system (1.7).

From the work by Brauer (1978), it is known that the asymptotic stability of solution $X=0$ of the system

$$
\frac{d X(t)}{d t}=F(t, X)+\int_{0}^{t} G(t-s) X(s) d s, \quad X(0)=\xi,
$$

is equivalent to the integrability of the resolvent kernel of the linear system. It means, it turns out to be equivalent to the non-vanishing of $\operatorname{det}(\lambda I-A-\hat{B}(\lambda))$ for $\operatorname{Re} \lambda \geq 0$, that is, there is no solutions in the right half plane $\operatorname{Re} \lambda \geq 0$ of the

$$
\operatorname{det}(\lambda I-A-\hat{B}(\lambda))=0
$$

where 2.19) is the characteristic equation for a system, $I$ is the identity matrix, $A$ is the Jacobian matrix of the system in relation to the variable $X, \hat{B}(\lambda)=\int_{0}^{\infty} e^{(-\lambda s)} B(s) d s$ and, $B$ is the Jacobian matrix of the system in relation to the variable $Y=X(t-s)$ with time delay. 
For the purpose of finding the stability of the solutions of the system (1.7) we follow the idea found in the study by Brauer (1978) in the section 3, denoting the limiting system (2.4) as the unperturbed system. Thus, the equilibriums of the limiting system need to be found and the hypothesis $(2.6)$ and $(2.9)$ need to be true.

Since we have already calculated the equilibriums of the limiting system (unperturbed system) in the previous section, and the perturbation function of the system tends to zero as $t$ goes to infinity, we are going to use the linearization theory for the limiting system.

Then, the stability of the equilibriums of the limiting system is a consequence of stability of the zero solution of the linearized system. Considering the assumptions in (2.6) true, which lead to a hypothesis (2.9), the perturbation function of the system is integrable, hence we have the necessary assumptions to use the theorem 2 in the work from $\operatorname{Brauer}(1978)$, as well as showing that if the zero solution of the linearized limiting system is uniformly asymptotically stable, then the solution of the delay system will be also stable.

\subsubsection{Stability of the Equilibriums of the Limiting System}

Once we remind the limiting system (or the unperturbed system as Brauer (1978) named) (2.4), we regard

$$
H(t)=\int_{t}^{\infty} \gamma Y(s) P_{t}(t-s) d s
$$

as a perturbation, where $H$ is a matrix, $Y$ is the matrix containing the variables of the populations, $P_{t}$ is the vector containing the function $P_{t}^{i}(t-s) e^{-d(t-s)}$. Adding the perturbation with the limiting system we have the initial system (1.7).

We must remember that we have a Disease Free equilibrium (DFE) of the system (2.4) which is given by $D_{0}=\left(N^{*}, 0,0,0,0,0,0,0,0\right)$. We want to investigate the stability of DFE $D_{0}$, linearizing the system at $D_{0}$, by writing the solution like

$$
\begin{aligned}
S(t) & =N^{*}+s(t), & I_{i}(t)=0+y_{i}(t) \\
C_{i}(t) & =0+c_{i}(t), & R_{i}(t)=0+r_{i}(t) \\
I_{j i}(t) & =0+k_{i}(t), &
\end{aligned}
$$

for $i \neq j, i, j=1,2$. Then, the null solution of the associated linear system 


$$
\begin{aligned}
& s^{\prime}(t)=-d s(t)-\overline{\beta_{1}} N^{*}\left(y_{1}(t)+k_{1}(t)\right)-\overline{\beta_{2}} N^{*}\left(y_{2}(t)+k_{2}(t)\right) \\
& y_{1}^{\prime}(t)=-(d+\gamma) y_{1}(t)+\overline{\beta_{1}} N^{*}\left(y_{1}(t)+k_{1}(t)\right) \\
& y_{2}^{\prime}(t)=-(d+\gamma) y_{2}(t)+\overline{\beta_{2}} N^{*}\left(y_{2}(t)+k_{2}(t)\right) \\
& c_{1}^{\prime}(t)=\gamma y_{1}(t)-d c_{1}(t)+\gamma \int_{0}^{\infty} y_{1}(t-z) P_{z}^{1}(z) e^{-d(z)} d z \\
& c_{2}^{\prime}(t)=\gamma y_{2}(t)-d c_{2}(t)+\gamma \int_{0}^{\infty} y_{2}(t-z) P_{z}^{2}(z) e^{-d(z)} d z \\
& r_{1}^{\prime}(t)=-d r_{1}(t)-\gamma \int_{0}^{\infty} y_{1}(t-z) P_{z}^{1}(z) e^{-d(z)} d z \\
& r_{2}^{\prime}(t)=-d r_{2}(t)-\gamma \int_{0}^{\infty} y_{2}(t-z) P_{z}^{2}(z) e^{-d(z)} d z \\
& k_{2}^{\prime}(t)=-(d+\gamma) k_{2}(t) \\
& k_{1}^{\prime}(t)=-(d+\gamma) k_{1}(t)
\end{aligned}
$$

corresponds to the solution $D_{0}$ of the system (2.4).

Therefore, finding the exponential solution for the associated linear system is to determine the exponential rate of growth or decay $\lambda \in \mathcal{C}$ such that $\lambda$ is the root of the characteristic equation

$$
\operatorname{det}\left(\lambda I-H_{0}-\hat{G}(\lambda)\right)=0
$$

where $I$ is the identity matrix $9 \times 9, H_{0}$ is the matrix

$$
H_{0}=\left[\begin{array}{ccccccccc}
-d & -\overline{\beta_{1}} N^{*} & -\overline{\beta_{2}} N^{*} & 0 & 0 & 0 & 0 & -\overline{\beta_{2}} N^{*} & -\overline{\beta_{1}} N^{*} \\
0 & \overline{\beta_{1}} N^{*}-(d+\gamma) & 0 & 0 & 0 & 0 & 0 & 0 & \overline{\beta_{1}} N^{*} \\
0 & 0 & \overline{\beta_{2}} N^{*}-(d+\gamma) & 0 & 0 & 0 & 0 & \overline{\beta_{2}} N^{*} & 0 \\
0 & \gamma & 0 & -d & 0 & 0 & 0 & 0 & 0 \\
0 & 0 & \gamma & 0 & -d & 0 & 0 & 0 & 0 \\
0 & 0 & 0 & 0 & 0 & -d & 0 & 0 & 0 \\
0 & 0 & 0 & 0 & 0 & 0 & -d & 0 & 0 \\
0 & 0 & 0 & 0 & 0 & 0 & 0 & -(d+\gamma) & 0 \\
0 & 0 & 0 & 0 & 0 & 0 & 0 & 0 & -(d+\gamma)
\end{array}\right],
$$


and, the matrix

$$
\hat{G}(\lambda)=\left[\begin{array}{ccccccccc}
0 & 0 & 0 & 0 & 0 & 0 & 0 & 0 & 0 \\
0 & 0 & 0 & 0 & 0 & 0 & 0 & 0 & 0 \\
0 & 0 & 0 & 0 & 0 & 0 & 0 & 0 & 0 \\
0 & \gamma \hat{G}_{1}(\lambda) & 0 & 0 & 0 & 0 & 0 & 0 & 0 \\
0 & 0 & \gamma \hat{G}_{2}(\lambda) & 0 & 0 & 0 & 0 & 0 & 0 \\
0 & -\gamma \hat{G}_{1}(\lambda) & 0 & 0 & 0 & 0 & 0 & 0 & 0 \\
0 & 0 & -\gamma \hat{G}_{2}(\lambda) & 0 & 0 & 0 & 0 & 0 & 0 \\
0 & 0 & 0 & 0 & 0 & 0 & 0 & 0 & 0 \\
0 & 0 & 0 & 0 & 0 & 0 & 0 & 0 & 0
\end{array}\right],
$$

where, $\hat{G}_{i}(\lambda)=\int_{0}^{\infty} e^{-\lambda z} g_{i}(z) d z=\int_{0}^{\infty} e^{-\lambda z} P_{z}^{i}(z) e^{-d z} d z$, it means $\hat{G}(\lambda)$ is the matrix whose the elements are the Laplace transform of the continuous function $g_{i}(z)=e^{-d z} P_{z}^{i}(z)$.

As soon as we solve the characteristic equation (2.21) of the associated system, we have the following eigenvalues of the system at DFE

$$
\begin{array}{ll}
\lambda_{1}=-d, & \lambda_{6}=-(d+\gamma) \\
\lambda_{2}=-d, & \lambda_{7}=-(d+\gamma) \\
\lambda_{3}=-d, & \lambda_{8}=-(d+\gamma)+\overline{\beta_{1}} N^{*} \\
\lambda_{4}=-d, & \lambda_{9}=-(d+\gamma)+\overline{\beta_{2}} N^{*} \\
\lambda_{5}=-d . &
\end{array}
$$

It gives us the following theorem about the stability of Disease Free equilibrium.

Theorem 4. If $\mathcal{R}_{0}<1$ then the Disease Free equilibrium, $D_{0}=\left(N^{*}, 0,0,0,0,0,0,0,0\right)$, of the system (2.4) is locally asymptotically stable. And $D_{0}$ will be unstable if $\mathcal{R}_{1}>1$ or $\mathcal{R}_{2}>1$.

Proof. If $\frac{N^{*} \overline{\beta_{1}}}{d+\gamma}<1$ and $\frac{N^{*} \overline{\beta_{2}}}{d+\gamma}<1$, all the eigenvalues given by 2.22 are negative. Then $D_{0}$ is locally stable. In case of $\frac{N^{*} \overline{\beta_{1}}}{d+\gamma}>1$ or $\frac{N^{*} \overline{\beta_{2}}}{d+\gamma}>1$, the eigenvalues $\lambda_{8}$ or $\lambda_{9}$ are positive, consequently, $D_{0}$ will be unstable.

Now we want to know about the stability of the Boundary equilibriums of the system (2.4) which is given by

$$
D_{1}=\left(\frac{d+\gamma}{\overline{\beta_{1}}}, \frac{d}{\overline{\beta_{1}}}\left[\frac{\overline{\beta_{1}} N^{*}}{d+\gamma}-1\right], 0, \frac{\gamma}{d}\left(1-M_{1}\right) I_{1}^{*}, 0, M_{1} \frac{\gamma}{\overline{\beta_{1}}}\left[\frac{\overline{\beta_{1}} N^{*}}{d+\gamma}-1\right], 0,0,0\right)
$$


and,

$$
D_{2}=\left(\frac{d+\gamma}{\overline{\beta_{2}}}, 0, \frac{d}{\overline{\beta_{2}}}\left[\overline{\frac{\beta_{2}}{d+\gamma}}-1\right], 0, \frac{\gamma}{d}\left(1-M_{2}\right) I_{2}^{*}, 0, M_{2} \frac{\gamma}{\overline{\beta_{2}}}\left[\frac{\overline{\beta_{2}} N^{*}}{\gamma+d}-1\right], 0,0\right) .
$$

We want to investigate the stability of the Boundary equilibrium $D_{1}$ linearizing the system at $D_{1}$, by writing the solution like

$$
\begin{aligned}
S(t) & =S^{*}+s(t), & I_{1}(t) & =I_{1}^{*}+y_{1}(t) \\
C_{1}(t) & =C_{1}^{*}+c_{1}(t), & R_{1}(t) & =R_{1}^{*}+r_{1}(t) \\
I_{j i}(t) & =0+k_{i}(t), & I_{2}(t) & =0+y_{2}(t) \\
C_{2}(t) & =0+c_{2}(t), & R_{2}(t) & =0+r_{2}(t)
\end{aligned}
$$

for $i \neq j, i, j=1,2$. Then, the system (2.4) is associated with the linear system

$$
\begin{aligned}
& s^{\prime}(t)=-d s(t)-\overline{\beta_{1}} S^{*}\left(y_{1}(t)+k_{1}(t)\right)-\overline{\beta_{1}} s(t) I^{*}-\overline{\beta_{2}} S^{*}\left(y_{2}(t)+k_{2}(t)\right) \\
& y_{1}^{\prime}(t)=-(d+\gamma) y_{1}(t)+\overline{\beta_{1}} S^{*}\left(y_{1}(t)+k_{1}(t)\right)+\overline{\beta_{1}} s(t) I_{1}^{*} \\
& y_{2}^{\prime}(t)=-(d+\gamma) y_{2}(t)+\overline{\beta_{2}} S^{*}\left(y_{2}(t)+k_{2}(t)\right) \\
& c_{1}^{\prime}(t)=\gamma y_{1}(t)-d c_{1}(t)+\gamma \int_{0}^{\infty} y_{1}(t-z) P_{z}^{1}(z) e^{-d(z)} d z \\
& c_{2}^{\prime}(t)=\gamma y_{2}(t)-d c_{2}(t)+\gamma \int_{0}^{\infty} y_{2}(t-z) P_{z}^{2}(z) e^{-d(z)} d z \\
& r_{1}^{\prime}(t)=-d r_{1}(t)-\overline{\alpha_{2}} \phi R_{1}^{*}\left(y_{2}(t)+k_{2}(t)\right)-\gamma \int_{0}^{\infty} y_{1}(t-z) P_{z}^{1}(z) e^{-d(z)} d z \\
& r_{2}^{\prime}(t)=-d r_{2}(t)-\overline{\alpha_{1}} \phi r_{2}(t) I_{1}^{*}-\gamma \int_{0}^{\infty} y_{2}(t-z) P_{z}^{2}(z) e^{-d(z)} d z \\
& k_{2}^{\prime}(t)=-(d+\gamma) k_{2}(t)+\overline{\alpha_{2}} \phi R_{1}^{*}\left(y_{2}(t)+k_{2}(t)\right) \\
& k_{1}^{\prime}(t)=-(d+\gamma) k_{1}(t)+\overline{\alpha_{1}} \phi I_{1}^{*} r_{2}(t) .
\end{aligned}
$$

Therefore, finding the exponential solution for the associated linear system is to determine the exponential rate of growth or decay $\lambda \in \mathcal{C}$ such that $\lambda$ is the root of the characteristic equation

$$
\operatorname{det}\left(\lambda I-H_{1}-\hat{G}(\lambda)\right)=0
$$

where $I$ is the identity matrix $9 \times 9, H_{1}$ is the matrix 


$$
H_{1}=\left[\begin{array}{ccccccccc}
-d \mathcal{R}_{1} & -(d+\gamma) & -\overline{\beta_{2}} \frac{d+\gamma}{\beta_{1}} & 0 & 0 & 0 & 0 & -\overline{\beta_{2}} \frac{d+\gamma}{\bar{\beta}} & -(d+\gamma) \\
d\left(\mathcal{R}_{1}-1\right) & 0 & 0 & 0 & 0 & 0 & 0 & 0 & (d+\gamma) \\
0 & 0 & (d+\gamma)\left(\frac{\overline{\beta_{2}}}{\beta_{1}}-1\right) & 0 & 0 & 0 & 0 & \overline{\beta_{2}} \frac{d+\gamma}{\overline{\beta_{1}}} & 0 \\
0 & \gamma & 0 & -d & 0 & 0 & 0 & 0 & 0 \\
0 & 0 & \gamma & 0 & -d & 0 & 0 & 0 & 0 \\
0 & 0 & -\frac{\overline{\alpha_{2}} \phi}{\overline{\beta_{1}}} \gamma M_{1}\left(\mathcal{R}_{1}-1\right) & 0 & 0 & -d & 0 & -\frac{\overline{\alpha_{2}} \phi}{\overline{\beta_{1}}} \gamma M_{1}\left(\mathcal{R}_{1}-1\right) & 0 \\
0 & 0 & 0 & 0 & 0 & 0 & -d-\frac{\overline{\alpha_{1}} \phi}{\bar{\beta}_{1}} d\left(\mathcal{R}_{1}-1\right) & 0 & 0 \\
0 & 0 & \frac{\bar{\alpha}}{\overline{\beta_{1}} \phi} \gamma M_{1}\left(\mathcal{R}_{1}-1\right) & 0 & 0 & 0 & 0 & -(d+\gamma)+\frac{\overline{\alpha_{2}} \phi}{\bar{\beta}_{1}} \gamma M_{1}\left(\mathcal{R}_{1}-1\right) & 0 \\
0 & 0 & 0 & 0 & 0 & 0 & \frac{\bar{\alpha}}{\overline{\beta_{1}} \phi} d\left(\mathcal{R}_{1}-1\right) & 0 & -(d+\gamma)
\end{array}\right],
$$

and, the matrix

$$
\hat{G}(\lambda)=\left[\begin{array}{ccccccccc}
0 & 0 & 0 & 0 & 0 & 0 & 0 & 0 & 0 \\
0 & 0 & 0 & 0 & 0 & 0 & 0 & 0 & 0 \\
0 & 0 & 0 & 0 & 0 & 0 & 0 & 0 & 0 \\
0 & \gamma \hat{G}_{1}(\lambda) & 0 & 0 & 0 & 0 & 0 & 0 & 0 \\
0 & 0 & \gamma \hat{G}_{2}(\lambda) & 0 & 0 & 0 & 0 & 0 & 0 \\
0 & -\gamma \hat{G}_{1}(\lambda) & 0 & 0 & 0 & 0 & 0 & 0 & 0 \\
0 & 0 & -\gamma \hat{G}_{2}(\lambda) & 0 & 0 & 0 & 0 & 0 & 0 \\
0 & 0 & 0 & 0 & 0 & 0 & 0 & 0 & 0 \\
0 & 0 & 0 & 0 & 0 & 0 & 0 & 0 & 0
\end{array}\right],
$$

where, $\hat{G}_{i}(\lambda)=\int_{0}^{\infty} e^{-\lambda z} g_{i}(z) d z=\int_{0}^{\infty} e^{-\lambda z} P_{z}^{i}(z) e^{-d z} d z$, it means, $\hat{G}(\lambda)$ is the matrix whose the elements are the Laplace transform of the continuous function $g_{i}(z)=e^{-d z} P_{z}^{i}(z)$.

Once we solve the characteristic equation 2.23 ) of the associated system, we have the following eigenvalues

$$
\begin{array}{ll}
\lambda_{1}=-d, & \lambda_{6}=-d\left(1+\frac{\overline{\alpha_{1}} \phi}{\overline{\beta_{1}}}\left(\mathcal{R}_{1}-1\right)\right) \\
\lambda_{2}=-d, & \lambda_{7}=\frac{1}{2}\left(-d \mathcal{R}_{1}-\sqrt{\left(d \mathcal{R}_{1}\right)^{2}-4 d(d+\gamma)\left(\mathcal{R}_{1}-1\right)}\right) \\
\lambda_{3}=-d, & \lambda_{8}=\frac{1}{2}\left(-d \mathcal{R}_{1}+\sqrt{\left(d \mathcal{R}_{1}\right)^{2}-4 d(d+\gamma)\left(\mathcal{R}_{1}-1\right)}\right) \\
\lambda_{4}=-(d+\gamma), & \lambda_{9}=\frac{\overline{\alpha_{2}}}{\overline{\beta_{1}}} \phi \gamma M_{1}\left(\mathcal{R}_{1}-1\right)+\frac{\left(\overline{\beta_{2}}-\overline{\beta_{1}}\right)}{\overline{\beta_{1}}}(d+\gamma) \\
\lambda_{5}=-(d+\gamma) . &
\end{array}
$$

Now, we want to investigate the stability of the boundary equilibrium $D_{2}$ linearizing the system at $D_{2}$, by writing the solution like 


$$
\begin{aligned}
S(t) & =S^{*}+s(t), & I_{2}(t) & =I_{2}^{*}+y_{2}(t) \\
C_{2}(t) & =C_{2}^{*}+c_{2}(t), & R_{2}(t) & =R_{2}^{*}+r_{2}(t) \\
I_{j i}(t) & =0+k_{i}(t), & I_{1}(t) & =0+y_{1}(t) \\
C_{1}(t) & =0+c_{1}(t), & R_{1}(t) & =0+r_{1}(t)
\end{aligned}
$$

for $i \neq j, i, j=1,2$. Then, the system (2.4) is associated with the linear system

$$
\begin{aligned}
& s^{\prime}(t)=-d s(t)-\overline{\beta_{1}} S^{*}\left(y_{1}(t)+k_{1}(t)\right)-\overline{\beta_{2}} s(t) I_{2}^{*}-\overline{\beta_{2}} S^{*}\left(y_{2}(t)+k_{2}(t)\right) \\
& y_{1}^{\prime}(t)=-(d+\gamma) y_{1}(t)+\overline{\beta_{1}} S^{*}\left(y_{1}(t)+k_{1}(t)\right) \\
& y_{2}^{\prime}(t)=-(d+\gamma) y_{2}(t)+\overline{\beta_{2}} S^{*}\left(y_{2}(t)+k_{2}(t)\right)+\overline{\beta_{2}} s(t) I_{2}^{*} \\
& c_{1}^{\prime}(t)=\gamma y_{1}(t)-d c_{1}(t)+\gamma \int_{0}^{\infty} y_{1}(t-z) P_{z}^{1}(z) e^{-d(z)} d z \\
& c_{2}^{\prime}(t)=\gamma y_{2}(t)-d c_{2}(t)+\gamma \int_{0}^{\infty} y_{2}(t-z) P_{z}^{2}(z) e^{-d(z)} d z \\
& r_{1}^{\prime}(t)=-d r_{1}(t)-\overline{\alpha_{2}} \phi r_{1}(t) I_{2}^{*}-\gamma \int_{0}^{\infty} y_{1}(t-z) P_{z}^{1}(z) e^{-d(z)} d z \\
& r_{2}^{\prime}(t)=-d r_{2}(t)-\overline{\alpha_{1}} \phi R_{2}^{*}\left(y_{1}(t)+k_{1}(t)\right)-\gamma \int_{0}^{\infty} y_{2}(t-z) P_{z}^{2}(z) e^{-d(z)} d z \\
& k_{2}^{\prime}(t)=-(d+\gamma) k_{2}(t)+\overline{\alpha_{2}} \phi I_{2}^{*} r_{1}(t) \\
& k_{1}^{\prime}(t)=-(d+\gamma) k_{1}(t)+\overline{\alpha_{1}} \phi R_{2}^{*}\left(y_{1}(t)+k_{1}(t)\right) .
\end{aligned}
$$

Therefore, finding the exponential solution for the associated linear system is to determine the exponential rate of growth or decay $\lambda \in \mathcal{C}$ such that $\lambda$ is the root of the characteristic equation

$$
\operatorname{det}\left(\lambda I-H_{2}-\hat{G}(\lambda)\right)=0
$$

where $I$ is the identity matrix $9 \times 9, H_{2}$ is the matrix

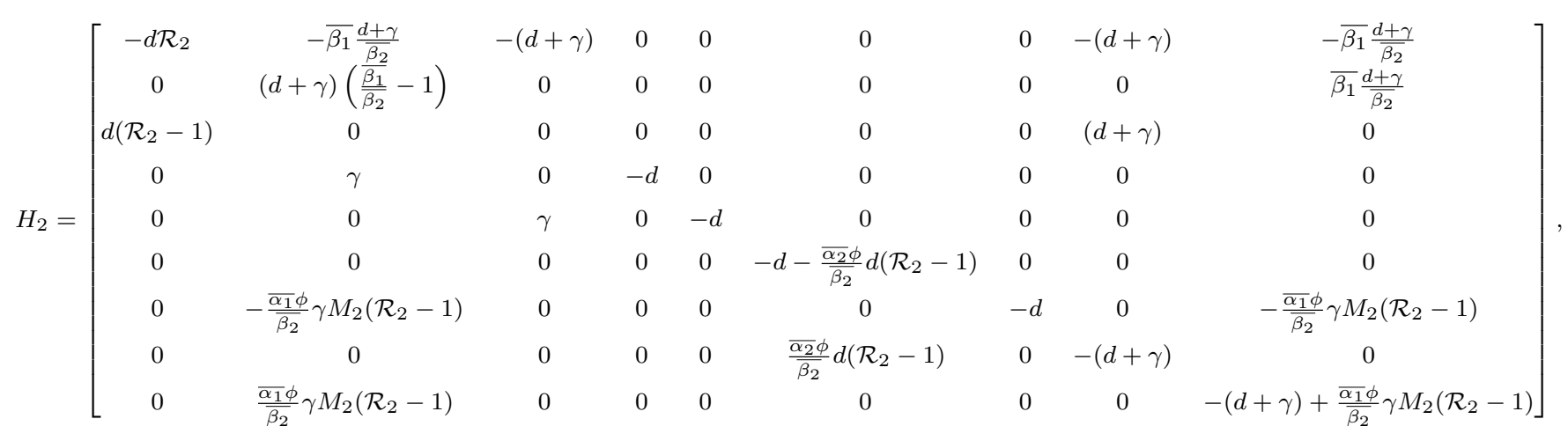


and, the matrix

$$
\hat{G}(\lambda)=\left[\begin{array}{ccccccccc}
0 & 0 & 0 & 0 & 0 & 0 & 0 & 0 & 0 \\
0 & 0 & 0 & 0 & 0 & 0 & 0 & 0 & 0 \\
0 & 0 & 0 & 0 & 0 & 0 & 0 & 0 & 0 \\
0 & \gamma \hat{G}_{1}(\lambda) & 0 & 0 & 0 & 0 & 0 & 0 & 0 \\
0 & 0 & \gamma \hat{G}_{2}(\lambda) & 0 & 0 & 0 & 0 & 0 & 0 \\
0 & -\gamma \hat{G}_{1}(\lambda) & 0 & 0 & 0 & 0 & 0 & 0 & 0 \\
0 & 0 & -\gamma \hat{G}_{2}(\lambda) & 0 & 0 & 0 & 0 & 0 & 0 \\
0 & 0 & 0 & 0 & 0 & 0 & 0 & 0 & 0 \\
0 & 0 & 0 & 0 & 0 & 0 & 0 & 0 & 0
\end{array}\right],
$$

where, $\hat{G}_{i}(\lambda)=\int_{0}^{\infty} e^{-\lambda z} g_{i}(z) d z=\int_{0}^{\infty} e^{-\lambda z} P_{z}^{i}(z) e^{-d z} d z$, it means, $\hat{G}(\lambda)$ is the matrix whose the elements are the Laplace transform of the continuous function $g_{i}(z)=e^{-d z} P_{z}^{i}(z)$.

Once we solve the characteristic equation 2.25) of the associated system, we have the following eigenvalues

$$
\begin{aligned}
& \lambda_{1}=-d \\
& \lambda_{2}=-d \\
& \lambda_{3}=-d \\
& \lambda_{4}=-(d+\gamma) \\
& \lambda_{5}=-(d+\gamma) \\
& \lambda_{6}=-d\left(1+\frac{\overline{\alpha_{2}} \phi}{\overline{\beta_{2}}}\left(\mathcal{R}_{2}-1\right)\right) \\
& \lambda_{7}=\frac{1}{2}\left(-d \mathcal{R}_{2}-\sqrt{\left(d \mathcal{R}_{2}\right)^{2}-4 d(d+\gamma)\left(\mathcal{R}_{2}-1\right)}\right) \\
& \lambda_{8}=\frac{1}{2}\left(-d \mathcal{R}_{2}+\sqrt{\left(d \mathcal{R}_{2}\right)^{2}-4 d(d+\gamma)\left(\mathcal{R}_{2}-1\right)}\right) \\
& \lambda_{9}=\frac{\overline{\alpha_{1}} \phi}{\overline{\beta_{2}}} \gamma M_{2}\left(\mathcal{R}_{2}-1\right)+\frac{\left(\overline{\beta_{1}}-\overline{\beta_{2}}\right)}{\overline{\beta_{2}}}(d+\gamma) .
\end{aligned}
$$

It gives us the following theorem about the stability of the Boundary equilibriums.

Theorem 5. The Boundary equilibrium, $D_{1}$, of the system (2.4) is always unstable in $\Omega_{X}$ region. And, $D_{2}$ is locally stable, in $\Omega_{X}$, if $\mathcal{R}_{\text {Inv }}<1$ and, is unstable in $\Omega_{X}$ if $\mathcal{R}_{\text {Inv }}>1$, where

$$
\mathcal{R}_{\text {Inv }}=\frac{\mathcal{R}_{1}}{\mathcal{R}_{2}}+\left(\mathcal{R}_{2}-1\right) \frac{\overline{\alpha_{1}} \phi \gamma M_{2}}{\overline{\beta_{2}}(d+\gamma)}
$$


Proof. Since $D_{1}$ is in $\Omega$ we have that $\mathcal{R}_{1}>1$. Then, $\mathcal{R}_{1}-1>0$, therefore, the eigenvalues $\lambda_{6}, \lambda_{7}$ and $\lambda_{8}$ in 2.24 are negative. However, $\lambda_{9}$ given in 2.24 is positive, since $\overline{\beta_{2}}>\overline{\beta_{1}}$ and $\mathcal{R}_{1}-1>0$. Thus, $D_{1}$ will be unstable.

For the analysis of the stability of the $D_{2}$, it is important to note that, since $D_{2}$ is in $\Omega, \mathcal{R}_{2}>1$ and, because of that, the eigenvalue in 2.26$) \lambda_{6}, \lambda_{7}$ and $\lambda_{8}$ are negative. Now, rewriting $\lambda_{9}$ which is given in 2.26 we obtain

$$
\begin{aligned}
\lambda_{9} & =\frac{\overline{\alpha_{1}} \phi \gamma M_{2}\left(\mathcal{R}_{2}-1\right)}{\overline{\beta_{2}}}+\frac{\overline{\beta_{1}}}{\overline{\overline{\beta_{2}}}}(d+\gamma)-(d+\gamma) \\
& =\frac{\overline{\alpha_{1}} \phi \gamma M_{2}}{\overline{\beta_{2}}}\left(\mathcal{R}_{2}-1\right)+\frac{\mathcal{R}_{1}}{\mathcal{R}_{2}}(d+\gamma)-(d+\gamma) .
\end{aligned}
$$

Thus $\lambda_{9}<0$, if $\mathcal{R}_{\text {Inv }}=\frac{\mathcal{R}_{1}}{\mathcal{R}_{2}}+\left(\mathcal{R}_{2}-1\right) \frac{\overline{\alpha_{1}} \phi \gamma M_{2}}{\overline{\beta_{2}}(d+\gamma)}<1$. Thus, the equilibrium $D_{2}$ will be locally stable.

will be unstable.

And $\lambda_{9}>0$, if $\mathcal{R}_{\text {Inv }}=\frac{\mathcal{R}_{1}}{\mathcal{R}_{2}}+\left(\mathcal{R}_{2}-1\right) \frac{\overline{\alpha_{1}} \phi \gamma M_{2}}{\overline{\beta_{2}}(d+\gamma)}>1$. Thus, the equilibrium $D_{2}$

In addition, if $\mathcal{R}_{\text {Inv }}=1, \quad S_{\min }=\frac{d+\gamma}{\beta_{2}}$ will be the root of the polynomial $O(S)$, the Boundary equilibrium will be the only positive root of the polynomial (as described before in details in the theorem for the existence of the equilibriums, in the previously section).

We can rewrite the threshold value $\mathcal{R}_{\text {Inv }}$ defined in the theorem above as

$$
\begin{aligned}
\mathcal{R}_{\text {Inv }} & =\frac{\mathcal{R}_{1}}{\mathcal{R}_{2}}+\left(\mathcal{R}_{2}-1\right) \frac{\overline{\alpha_{1}} \phi \gamma M_{2}}{\overline{\beta_{2}}(d+\gamma)} \\
& =\frac{\mathcal{R}_{1}}{\mathcal{R}_{2}}+\frac{\overline{\alpha_{1}} \phi}{\overline{\beta_{1}}} \frac{\gamma}{(d+\gamma)} M_{2} \mathcal{R}_{1}\left(1-\frac{1}{\mathcal{R}_{2}}\right) .
\end{aligned}
$$

And, we know from (2.8) that $1-M_{2}=d N_{2}>0$, which means that $M_{2}<1$. Then, if $\frac{\overline{\alpha_{1}} \phi}{\overline{\beta_{1}}} \leq 1$, we have that $\mathcal{R}_{I n v}<\mathcal{R}_{1}$. Biologically speaking this results mean that there is a range of values for $\beta_{1}$ for which the strain one cannot invade the population if the strain two is endemic. This way, the infection forces two may protect the population from infection forces one. After this range value, the infection forces start to coexist.

The value $\mathcal{R}_{\text {Inv }}$ is also called the Invasion Reproduction Number and we are going to discuss about this threshold further, over the next chapters.

The results for existence and stability of the equilibriums were proved assuming $\beta_{2}>$ $\beta_{1}$. For $\beta_{1}<\beta_{2}$ the results follow the same, but using $\mathcal{R}_{0}=\mathcal{R}_{1}$ and $R_{\text {Inv }}=\frac{\mathcal{R}_{2}}{\mathcal{R}_{1}}+\frac{\overline{\alpha_{2}} \phi}{\bar{\beta}_{2}} \frac{\gamma}{(d+\gamma)} M_{1} \mathcal{R}_{2}\left(1-\frac{1}{\mathcal{R}_{1}}\right)$. 
Therefore, the boundary equilibrium associated with the smaller infection rate will always be unstable, while the other one will depend on the $\mathcal{R}_{\text {Inv }}$.

\subsubsection{Symmetric Case}

For the analysis of the equilibriums stability on the symmetric case, where $\overline{\beta_{1}}=\overline{\beta_{2}}=$ $\beta, \overline{\alpha_{1}}=\overline{\alpha_{2}}=\alpha$ and $P^{1}(t)=P^{2}(t)$ which implies that $M_{1}=M_{2}=M$, we just need to solve the characteristic equation 2.21,

$$
\operatorname{det}\left(\lambda I-H_{0}-\hat{G}(\lambda)\right)=0
$$

of the linear associated system, taking equal parameters.

As soon as we solve this characteristic equation, we have the following eigenvalues of the linear associated system at $D_{0}$, for the symmetric case

$$
\begin{array}{ll}
\lambda_{1}=-d, & \lambda_{6}=-(d+\gamma) \\
\lambda_{2}=-d, & \lambda_{7}=-(d+\gamma) \\
\lambda_{3}=-d, & \lambda_{8}=-(d+\gamma)+\bar{\beta} N^{*} \\
\lambda_{4}=-d, & \lambda_{9}=-(d+\gamma)+\bar{\beta} N^{*} \\
\lambda_{5}=-d . &
\end{array}
$$

In the same way, taking the equal parameters in the matrices of the characteristic equations

$$
\operatorname{det}\left(\lambda I-H_{i}-\hat{G}(\lambda)\right)=0
$$

for $i=1,2$, the eigenvalues of the linear associated system at

$$
D_{1}=\left(\frac{d+\gamma}{\beta}, \frac{d}{\beta}\left(\mathcal{R}_{0}-1\right), 0, \frac{\gamma}{d}(1-M) I_{1}^{*}, 0, M \frac{\gamma}{\beta}\left(\mathcal{R}_{0}-1\right), 0,0,0\right)
$$

and, at

$$
D_{2}=\left(\frac{d+\gamma}{\beta}, 0, \frac{d}{\beta}\left(\mathcal{R}_{0}-1\right), 0, \frac{\gamma}{d}(1-M) I_{2}^{*}, 0, M \frac{\gamma}{\beta}\left(\mathcal{R}_{0}-1\right), 0,0\right),
$$


are the same and given by

$$
\begin{aligned}
& \lambda_{1}=-d \\
& \lambda_{2}=-d \\
& \lambda_{3}=-d \\
& \lambda_{4}=-(d+\gamma) \\
& \lambda_{5}=-(d+\gamma) \\
& \lambda_{6}=-d\left(1+\frac{\alpha \phi}{\beta}\left(\mathcal{R}_{0}-1\right)\right) \\
& \lambda_{7}=\frac{1}{2}\left(-d \mathcal{R}_{0}-\sqrt{\left(d \mathcal{R}_{0}\right)^{2}-4 d(d+\gamma)\left(\mathcal{R}_{0}-1\right)}\right) \\
& \lambda_{8}=\frac{1}{2}\left(-d \mathcal{R}_{0}+\sqrt{\left(d \mathcal{R}_{0}\right)^{2}-4 d(d+\gamma)\left(\mathcal{R}_{0}-1\right)}\right) \\
& \lambda_{9}=\frac{\alpha \phi}{\beta} \gamma M\left(\mathcal{R}_{0}-1\right) .
\end{aligned}
$$

It gives the following theorem about the stability of the equilibriums.

Theorem 6. If $\mathcal{R}_{0}<1$ then the Disease Free equilibrium of the system (2.4) is locally asymptotically stable in the symmetric case. It is unstable if $\mathcal{R}_{0}>1$. In addition, the Boundary equilibriums, $D_{1}$ and $D_{2}$, given in (2.30) and (2.31), respectively, are unstable in the $\Omega_{X}$ region, when $\mathcal{R}_{0}>1$.

Proof. If $\mathcal{R}_{0}<1$, then, $N^{*} \beta<d+\gamma$. Therefore, the eigenvalues $\lambda_{8}$ and $\lambda_{9}$ in 2.29) are also negative. It proves the local asymptotic stability of DFE. If $\mathcal{R}_{0}>1$, then $N^{*} \beta>d+\gamma$. And, the eigenvalues $\lambda_{8}$ and $\lambda_{9}$ in $(2.29)$ are positive, what proves the instability of the DFE.

According to the previous theorem, $D_{1}$ and $D_{2}$ are in the interest region $\Omega$ if $\mathcal{R}_{0}>1$. Then, $N^{*} \beta>d+\gamma$ and the eigenvalue $\lambda_{6}$, given in 2.32 is negative. Also, the eigenvalues $\lambda_{7}$ and $\lambda_{8}$ have negative real part, but $\lambda_{9}$ is positive, since $\mathcal{R}_{0}>1$. This way, the Boundary equilibriums are always unstable.

It is important to note that, when $\mathcal{R}_{0}=1$, we have $\frac{d+\gamma}{\beta}=N^{*}$. Therefore, the only equilibrium of the system is the Disease Free equilibrium in this case. Biologically speaking, these results mean that, since the serotypes have the same force of infection, if one strain invades a disease free population, strain two does the same at the same time. Thus, the two infection starts to coexist in a susceptible population at the same time. 


\subsubsection{Stability of the solutions of the Time Delay System}

Over previous section, we have shown the equilibriums of the unperturbed (limiting) system (2.4) and the stability of the equilibriums. In addition, the assumptions in (2.6) about kernel function are true, which lead to

$$
\int_{0}^{\infty} P_{s}^{i}(s) e^{-d s} d s<\infty .
$$

Also, leading to the assumption (2.9), that is,

$$
\int_{0}^{\infty} s P_{s}^{i}(s) e^{-d s} d s<\infty
$$

Moreover, with the assumption 2.9 , we can prove that the perturbation function $H(t)$ defined in 2.20 is integrable. In fact, integrating $H(t)$, assuming that $Y$ is a bounded function, changing the integration limiting and using 2.9) we have

$$
\int_{0}^{\infty} H(t)<\infty
$$

Also, the theorems proved in the previous section show the uniformly asymptotic stability of the zero solution of the linear limiting system, therefore the stability of the equilibriums of the limiting system.

By theorem 2 from Brauer (1978) (see Appendix) we have the following results about the solutions stability of the initial system $(1.7)$.

Corollary 1. If $\mathcal{R}_{1}<1$ and $\mathcal{R}_{2}<1$ then the Disease Free equilibrium, $D_{0}=\left(N^{*}, 0,0,0,0,0,0,0,0\right)$, of the system (1.7) is locally asymptotically stable. And, $D_{0}$ is unstable if $\mathcal{R}_{1}>1$ or $\mathcal{R}_{2}>1$.

Corollary 2. Without loss of generality, we suppose $\overline{\beta_{2}}>\overline{\beta_{1}}$. Then, the Boundary equilibrium, $D_{1}$, of the system (1.7) is always unstable in $\Omega . D_{2}$ is locally stable in $\Omega$ if $\mathcal{R}_{\text {Inv }}<1$ and, it is unstable in $\Omega$ if $\mathcal{R}_{\text {Inv }}>1$, where

$$
\mathcal{R}_{\text {Inv }}=\frac{\mathcal{R}_{1}}{\mathcal{R}_{2}}+\left(\mathcal{R}_{2}-1\right) \frac{\overline{\alpha_{1}} \phi \gamma M_{2}}{\overline{\beta_{2}}(d+\gamma)} .
$$

\section{Symmetric Case}

Corollary 3. If $\mathcal{R}_{0}<1$ then the Disease Free equilibrium of the system (1.7) is locally asymptotically stable in the symmetric case. And, it is unstable if $\mathcal{R}_{0}>1$. In addition, the Boundary equilibriums, $D_{1}$ and $D_{2}$, given in (2.30) and (2.31), respectively, are unstable in $\Omega$, when $\mathcal{R}_{0}>1$. 


\subsection{Global Stability}

Over the previous sections, we have seen the set $\Omega=\left\{\left(S, I_{1}, I_{2}, C_{1}, C_{2}, R_{1}, R_{2}, I_{12}, I_{21}, R\right) \in\right.$ $\mathbb{R}_{+}^{10}$ such that $\left.S+I_{1}+I_{2}+C_{1}+C_{2}+R_{1}+R_{2}+I_{12}+I_{21}+R \leq N^{*}\right\}$ is positively invariant for the system (1.7). Also, we have proved the local stability for the DFE equilibrium and for the Boundary Endemic equilibrium which is completely determined by the Reproduction numbers and by the Invasion Reproduction number. In this section, we are going to investigate the global dynamics of the system (1.7), by constructing suitable Lyapunov functions. The results are going to be established and formalized in the following theorems.

\subsubsection{Global Stability of the DFE}

Theorem 7. If $\mathcal{R}_{0} \leq 1$ then the Disease-Free equilibrium $D_{0}$ of the system (1.7) is globally attractive in $\Omega$.

Proof. First, we denote the positive function, for $i=1,2$,

$$
\Pi_{i}(t)=\int_{0}^{\infty} \gamma I_{i}(s) P^{i}(t-s) e^{-d(t-s)} d s
$$

It is considered $h(z)=z-1-\ln (z), z \in \mathbb{R}_{+}^{*}$. Then, $h \geq 0$, since it has a global minimum at $z=1$ and $h(1)=0$.

Thus, we let $L$ be the function

$$
L(t)=\frac{1}{d+\gamma}\left(N^{*} h\left(\frac{S}{N^{*}}\right)+I_{1}+I_{2}+I_{21}+I_{12}+R_{1}+R_{2}+\Pi_{1}+\Pi_{2}\right),
$$

this way,

$$
\begin{aligned}
L(t) & =\frac{1}{d+\gamma}\left(S-N^{*}-N^{*} \ln \left(\frac{S}{N^{*}}\right)+I_{1}+I_{2}+I_{21}+I_{12}+R_{1}+R_{2}\right) \\
& +\frac{1}{d+\gamma}\left(\int_{0}^{\infty} \gamma I_{1}(s) P^{1}(t-s) e^{-d(t-s)} d s+\int_{0}^{\infty} \gamma I_{2}(s) P^{2}(t-s) e^{-d(t-s)} d s\right)
\end{aligned}
$$

is well-posed, it is a Lyapunov function, with $L \geq 0$, where the equality is true if and only if $S=N^{*}$ and, $I_{i}=0, R_{i}=0, I_{i j}=0$ for $i, j=1,2$, since $N^{*} h\left(\frac{S}{N^{*}}\right) \geq 0$, for any $S>0$. 
Now, differentiating $L$ along the solution of the system (1.7) we have

$$
\begin{aligned}
L^{\prime} & =\frac{1}{d+\gamma}\left(S^{\prime}-N^{*} \frac{S^{\prime}}{S}+I_{1}^{\prime}+I_{2}^{\prime}+I_{21}^{\prime}+I_{12}^{\prime}+R_{1}^{\prime}+R_{2}^{\prime}+\Pi_{1}^{\prime}+\Pi_{2}^{\prime}\right) \\
& =\frac{1}{d+\gamma}\left(d N^{*}-d S-\frac{N^{*}}{S}\left(d N^{*}-d S-\beta_{1} S\left(I_{1}+I_{21}\right)-\beta_{2} S\left(I_{2}+I_{12}\right)\right)\right) \\
& +\frac{1}{d+\gamma}\left(-\gamma\left(I_{1}+I_{2}\right)-d\left(I_{1}+I_{2}\right)-d\left(R_{1}+R_{2}\right)-\int_{0}^{t} \gamma I_{1}(s) P^{1}{ }_{t}(t-s) e^{-d(t-s)} d s\right) \\
& +\frac{1}{d+\gamma}\left(-\int_{0}^{t} \gamma I_{2}(s) P^{2}{ }_{t}(t-s) e^{-d(t-s)} d s-d\left(I_{12}+I_{21}\right)-\gamma\left(I_{12}+I_{21}\right)\right) \\
& +\frac{1}{d+\gamma}\left(\int_{0}^{\infty} \gamma I_{1}(s) P^{1}{ }_{t}(t-s) e^{-d(t-s)} d s+\int_{0}^{\infty} \gamma I_{2}(s) P^{2}{ }_{t}(t-s) e^{-d(t-s)} d s\right) \\
& +\frac{1}{d+\gamma}\left(-d\left(\int_{0}^{\infty} \gamma I_{1}(s) P^{1}(t-s) e^{-d(t-s)} d s+\int_{0}^{\infty} \gamma I_{2}(s) P^{2}(t-s) e^{-d(t-s)} d s\right)\right) \\
L^{\prime} & =\frac{1}{d+\gamma}\left(d N^{*}\left(2-\frac{S^{*}}{N^{*}}-\frac{N^{*}}{S}\right)\right) \\
& +\frac{1}{d+\gamma}\left[\left(-(d+\gamma)+\beta_{1} N^{*}\right)\left(I_{1}+I_{21}\right)+\left(-(d+\gamma)+\beta_{2} N^{*}\right)\left(I_{2}+I_{12}\right)\right] \\
& +\frac{1}{d+\gamma}\left(-d\left(R_{1}+R_{2}+\Pi_{1}+\Pi_{2}\right)\right) \\
& +\frac{1}{d+\gamma}\left(\int_{t}^{\infty} \gamma I_{1}(s) P^{1}{ }_{t}(t-s) e^{-d(t-s)} d s+\int_{t}^{\infty} \gamma I_{2}(s) P^{2}{ }_{t}(t-s) e^{-d(t-s)} d s\right)
\end{aligned}
$$

Since $\frac{S}{N^{*}}+\frac{N^{*}}{S} \geq 2$ we have that the term in 2.40 is always non positive in $\Omega$. Also, since $\mathcal{R}_{0} \leq 1$ we have that the term in 2.41) is always non positive in $\Omega . R_{1}$ and $R_{2}$ are in $\Omega$ and, $\Pi_{1}$ and $\Pi_{2}$ are positive functions of $t$, this way we have that the term in 2.42) is always non positive. The last term in 2.43 is non positive because $P(t)$ is decreasing and, thus $P^{i}{ }_{t}$ is negative.

Therefore, $L^{\prime} \leq 0$ in $\Omega$ and, the equality is true if and only if each term of the equation is zero. From (2.40) the equality is true if and only if $S=N^{*}$ and, with $\mathcal{R}_{0}<1$, from (2.41) to 2.43) we conclude that $I_{1}=I_{2}=R_{1}=R_{2}=C_{1}=C_{2}=I_{12}=I_{21}=0$.

In the case that $\mathcal{R}_{0}=1$, since $S=N^{*}$, from the first equation of the system we have that $S^{\prime}=-\beta_{i} N^{*}\left(I_{i}+I_{j i}\right)<0$. But this is a contradiction with the fact that, since $S=N^{*}$, it is implied that $S^{\prime}=0$. Thus $I_{i}+I_{j i}=0$. Therefore, for instance, if $\mathcal{R}_{2}=\mathcal{R}_{0}=1$ we still have that $I_{2}=0$ and $I_{12}=0$.

This way, defining $E=\left\{\left(S, I_{1}, I_{2}, C_{1}, C_{2}, R_{1}, R_{2}, I_{12}, I_{21}, R\right) \in \Omega ; L^{\prime}(t)=0\right\}$ the singleton $D_{0}$ is the largest invariant set in $E$. By the Invariance Principle for IDE (Burton, 2005; LaSalle, 1976) we have that the Disease Free equilibrium $D_{0}$ of the system (1.7) is globally asymptotically stable in $\Omega$. 


\subsubsection{Global Stability of the Boundary Equilibrium}

We have proved over previous section, that the Boundary equilibrium $D_{2}$ is locally asymptotically stable, when $\mathcal{R}_{2}>1$ and the Invasion Reproduction Number $\mathcal{R}_{\text {Inv }}$ is smaller than 1 . Now we are able to show that under these same conditions the trajectories with initials conditions in $\Omega-\left\{\left(S, I_{1}, I_{2}, C_{1}, C_{2}, R_{1}, R_{2}, I_{12}, I_{21}, R\right) \in \Omega ; I_{2}=0\right\}$ approach that equilibrium. To prove this fact, we firstly need the following result.

Lemma 1. If $\mathcal{R}_{2}>1$, and $\mathcal{R}_{\text {Inv }}<1$ then the trajectories of system (1.7) which start in $\Omega$ approach the invariant set $\Omega_{2}=\left\{\left(S, I_{1}, I_{2}, C_{1}, C_{2}, R_{1}, R_{2}, I_{12}, I_{21}, R\right) \in \Omega \quad ; I_{1}=C_{1}=R_{1}=\right.$ $\left.I_{12}=I_{21}=R=0\right\}$.

Proof. Considering the Lyapunov function

$$
L_{1}(t)=\Pi_{1}+R_{1}+I_{12}
$$

with $\Pi_{1}$ being the same function definite in (2.37).

Thus, differentiating $L_{1}$ along the solution of the system (1.7) we have

$$
\begin{aligned}
L_{1}^{\prime} & =\Pi_{1}^{\prime}+R_{1}^{\prime}+I_{12}^{\prime} \\
& =-d \Pi_{1}+\int_{0}^{\infty} \gamma I_{1}(t) P_{t}^{1}(t-s) e^{-d(t-s) d s}-d R_{1}-(d+\gamma) I_{12}-\int_{0}^{t} \gamma I_{1}(t) P_{t}^{1}(t-s) e^{-d(t-s) d s} \\
& =-d \Pi_{1}-\left(-\int_{t}^{\infty} \gamma I_{1}(t) P_{t}^{1}(t-s) e^{-d(t-s) d s}\right)-d R_{1}-(d+\gamma) I_{12}
\end{aligned}
$$

Therefore, $L_{1}^{\prime} \leq 0$ in $\Omega$ and, the equality is true if, and only if, $R_{1}=0, I_{12}=0$, $\Pi_{1}=0$ and $-\int_{t}^{\infty} \gamma I_{1}(t) P_{t}^{1}(t-s) e^{-d(t-s) d s}=0$. In addition, from the equation of the system and, from the equality above, we have directly that $I_{1}=0$ and, $C_{1}$ approach zero when $\mathrm{t}$ goes to infinity. Also, since $I_{1}=0$ we have that

$$
0=I_{1}^{\prime}(t)=\overline{\beta_{1}} S I_{21}
$$

Then, $S=0$ or $I_{21}=0$. If $S=0$ then for the first equation of the system we have

$$
S^{\prime}(t)=d N^{*}>0
$$

which is a contradiction, since $S=0$ implies $S^{\prime}=0$. Then $I_{21}=0$.

Therefore, we have shown that the maximal invariant set contained in set of all points in $\Omega$ where $L_{1}^{\prime}=0$ is $\Omega_{2}$. It shows the lemma. 
This lemma shows that under the conditions about the Reproduction numbers is sufficient to study the dynamics of the delay system (1.7) only on the projection of $\Omega_{2}$, it means that, in the set $\Omega_{2_{p}}=\left\{\left(S, I_{2}, C_{2}, R_{2}\right) ; S+I_{2}+C_{2}+R_{2} \leq N^{*}\right)$ in $\mathbb{R}^{4}$. In this set, the initial system is reduced to

$$
\begin{aligned}
& \frac{d S(t)}{d t}=d N^{*}-d S-\overline{\beta_{2}} S I \\
& \frac{d I(t)}{d t}=\overline{\beta_{2}} S I-(d+\gamma) I \\
& \frac{d C(t)}{d t}=\gamma I-d C+\int_{0}^{t} \gamma I(s) P_{t}^{2}(t-s) e^{-d(t-s)} d s \\
& \frac{d R(t)}{d t}=-d R-\int_{0}^{t} \gamma I(s) P_{t}^{2}(t-s) e^{-d(t-s)} d s .
\end{aligned}
$$

This system has two equilibriums $D_{0_{p}}=\left(N^{*}, 0,0,0\right)$ and $D_{2_{p}}=\left(\frac{d+\gamma}{\overline{\beta_{2}}}, \frac{d}{\overline{\beta_{2}}}\left(\mathcal{R}_{2}-1\right), C^{*}, R^{*}\right)$ correspondent to the projections of $D_{0}$ and $D_{2}$, respectively. Now we are going to prove that all solutions of the system 2.47 with initial conditions in $\Omega_{2_{p}}-\left\{(S, I, C, R) \in \mathbb{R}^{4} ; I=0\right\}$ approach the equilibrium $D_{2_{p}}$ when $\mathcal{R}_{2}>1$.

Theorem 8. If $\mathcal{R}_{2}>1$ then the Endemic equilibrium $D_{2_{p}}$ of the system $(2.47)$ is globally stable in $\Omega_{2_{p}}$.

Proof. The idea for this proof is the same idea used in O' Regan (2010). Once we let $\left(S^{*}, I^{*}, C^{*}, R^{*}\right)$ be the Endemic equilibrium of the system (2.47). This positive equilibrium exists when $\mathcal{R}_{2}>1$. At the equilibrium, the equalities

$$
\begin{array}{r}
d N^{*}=d S^{*}+\overline{\beta_{2}} S^{*} I^{*} \\
\overline{\beta_{2}} S^{*} I^{*}=(d+\gamma) I^{*} \\
\gamma I^{*}-d C^{*}-\gamma I^{*} M_{2}=0 \\
-d R^{*}+\gamma I^{*} M_{2}=0
\end{array}
$$

hold. Substituting these expressions into the system, we can rewrite the system in this form

$$
\begin{aligned}
& \frac{d S(t)}{d t}=-d\left(S-S^{*}\right)-\overline{\beta_{2}}\left(S I-S^{*} I^{*}\right) \\
& \frac{d I(t)}{d t}=\overline{\beta_{2}} I\left(S-S^{*}\right) \\
& \frac{d C(t)}{d t}=\gamma\left(I-I^{*}\right)-d\left(C-C^{*}\right)+\int_{0}^{t} \gamma I(s) P_{t}^{2}(t-s) e^{-d(t-s)} d s-\gamma I^{*} M_{2} \\
& \frac{d R(t)}{d t}=-d\left(R-R^{*}\right)-\int_{0}^{t} \gamma I(s) P_{t}^{2}(t-s) e^{-d(t-s)} d s-\gamma I^{*} M_{2}
\end{aligned}
$$


Considering the Lyapunov function

$$
L_{2}(t)=S-S^{*}-S^{*} \ln \left(\frac{S}{S^{*}}\right)+I-I^{*}-I^{*} \ln \left(\frac{I}{I^{*}}\right) .
$$

Now, differentiating $L_{2}$ along the solution of the system, we have

$$
\begin{aligned}
L_{2}^{\prime} & =S^{\prime}+I^{\prime}-S^{*} \frac{S^{\prime}}{S}-I^{*} \frac{I^{\prime}}{I} \\
& =\left(d S^{*}+\overline{\beta_{2}} S^{*} I^{*}\right)\left(2-\frac{S}{S^{*}}-\frac{S^{*}}{S}\right) .
\end{aligned}
$$

Therefore $L_{2}^{\prime} \leq 0$ in $\Omega_{2_{p}}$ and, the equality $L_{2}^{\prime}=0$ is true if, and only if $S=S^{*}$. Since $S=S^{*}$, we have $S^{\prime}=0$ and, from the first equation of the system $(2.48)$ implies that $I=I^{*}$. In this set, from the last equations of the system $(2.48)$ follows directly that $C$ approaches to $C^{*}$ and $R$ approaches to $R^{*}$ when $t \rightarrow \infty$. By a Invariance Principle for IDE (Burton, 2005; LaSalle, 1976) we have that the Endemic equilibrium $D_{2_{p}}$ is globally asymptotically stable in $\Omega_{2_{p}}$.

The local asymptotic stability of $D_{2}$, the lemma and the theorem above demonstrate the global stability of $D_{2}$ in $\Omega$ under the conditions $1<\mathcal{R}_{2}$ and $\mathcal{R}_{\text {Inv }}<1$. 


\section{CHAPTER 3}

\section{Numerical Analysis}

In this chapter, we are going to analyse numerically the solutions of the system in order to understand better the model as well as using the numerical approach to obtain information about the local stability of the Coexistence Endemic equilibrium and the local dynamics.

The numerical values for the parameters are shown on the table below. To model the infection, we have used the data taken from primary references about Dengue fever.

Table 3.1: Numerical values of the parameters

\begin{tabular}{llll}
\hline Parameter & Meaning & Value & Reference \\
\hline$\frac{1}{d}$ & life expectancy & $65-75$ years & (IBGE, 2018) \\
$\frac{1}{\gamma}$ & recovery time & $4-10$ days & (Gubler, 2014; WHO, 2009) \\
$\frac{1}{\omega_{i}}$ & cross immunity protection time & $2-9$ months & (Gubler, 2014; WHO, 2009) \\
$\beta_{i}$ & infection rate, serotype $i$ & $40-200$ & $\star$ \\
$\alpha_{i}$ & reinfection rate, serotype $i$ & $40-200$ & $\star$ \\
$\phi$ & ADE factor & $0-5$ & Ferguson, 1999a) \\
\hline
\end{tabular}

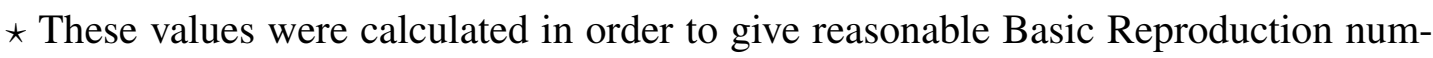
bers for Dengue. For instance, Maier (2017) and Massad (2001) have estimated the range of 
Table 3.2: Parameters value used in the simulations

\begin{tabular}{ll}
\hline Parameter & Simulation value \\
\hline$d$ & $0.015 y^{-1}$ \\
$\gamma$ & $52 y^{-1}$ \\
$\omega_{i}$ & $2 y^{-1}$ \\
$\phi$ & $0-5$ \\
$\beta_{i}$ & $40-200 \star$ \\
$\alpha_{i}$ & $\beta_{i}$ \\
\hline
\end{tabular}

$\mathcal{R}_{0}$ between 1.38 to 7.86 with Brazilian dataset. Reiner (2014) estimated the range of the Basic Reproduction number varied from 0.76 to over 5 from dataset from Peru and, with dataset from Thailand, Ferguson (1999b) estimated for 1.38 to 7.70, with average 3.2. Moreover, the interval of the values for the parameter is in accordance to the values found in the study by Kooi (2014), given by $2 \gamma$.

In the particular case, when we assume that the temporary immunity is exponentially distributed, in other words, $P^{i}(t)=e^{-\omega_{i} t}$, we have that $\frac{1}{\omega_{i}}$ is the average of the cross immunity time. In order to analyse numerically the general case when we assume a general function representing the cross immunity time, we will first define a function that satisfies the necessary conditions.

In this case, we need to choose a function, not necessarily a exponential function, which can represent the immunity period. Also, some mathematical and biological assumptions need to be addressed and satisfied, for each serotype $i=1,2$ :

$$
\begin{array}{r}
P^{i}(0)=1, \quad P^{i}(\infty)=0, \quad P^{i^{\prime}}(s)<0, \quad \text { and } \\
\int_{0}^{\infty} P^{i}(s) d s=\frac{1}{\omega_{i}}
\end{array}
$$

with $\omega_{i}$ given on the table 3.2 .

In order to simplify, it is chosen, $P^{i}(s)=P(s)$ for $i=1,2$ and we are going to estimate a cubic polynomial that satisfies the assumptions in 3.1 . We could choose any continuous decreasing function, as polynomials or combinations of polynomials, exponential, sinus and cosine functions, however we choose to work with a cubic polynomial for this numerical analysis, such as, $a s^{3}+b s^{2}+c s+d$ and, for each assumption, we will estimate the values $a, b, c$ and $d$. 
Besides that, in biological terms, it can be expected that the cross immunity time is a short term period that can not impact for longer than one year. Thus, we are going to assume that after one year, the immunity is not effective anymore and, after this period the individual is susceptible again, with average immunity time of 6 months, as in the particular case.

This way, we define

$$
P(s)= \begin{cases}a s^{3}+b s^{2}+c s+d, & 0 \leq s \leq 1 \\ 0, & s \geq 1\end{cases}
$$

meaning that for more than one year there is no more immunity cross period and during the first year a cubic polynomial represents the decreasing period for immunity.

Then, we want that $P(0)=1$ and $P(1)=0$. In addition, $P$ needs to be continuous at $s=1$, thus we need that $P^{\prime}(1)=0$ and since the average time is chosen to be six months we need that

$$
\int_{0}^{\infty} P(s) d s=\int_{0}^{1} P(s) d s=\frac{1}{\omega_{i}}=\frac{1}{2} .
$$

Substituting this assumptions on the function, the first one gives that $d=1$. Second assumption gives $a+b+c=-1$. The continuous assumption gives $3 a+2 b+c=0$. Substituting this equality and using (3.4) we can finally find that $a=2, b=-3$ and, $c=0$.

Thus, we are going to use that

$$
P(s)= \begin{cases}2 s^{3}-3 s^{2}+1, & 0 \leq s \leq 1 \\ 0, & s \geq 1\end{cases}
$$

with average time being $\frac{1}{2}$ year.

Thus, $N_{i}=\int_{0}^{\infty} P(s) e^{-d s} d s \approx 0.49$ and the value $M_{i}$ that appears in the equilibriums is given numerically by $M_{i}=\int_{0}^{\infty} P_{s}(s) e^{-d s} d s \approx 0.993$, with the parameters $d$ given on table (3.2). Note that $M_{i}=\frac{\omega_{i}}{\omega_{i}+d} \approx 0.993$ in the exponential case, meaning that the average time is held for both cases, particular and general.

Using these functions and values, we are going to explore and analyse the stability of the endemic equilibrium and the solutions of the IDE system with delay for that chosen function. 


\subsection{Stability of the Coexistence Endemic equilibrium}

\subsubsection{Symmetric case}

As we have seen in the chapter 3, the theorem ensures, in the symmetric case, that a unique endemic equilibrium with the coexistence of two serotypes within the invariant region always exist when the Basic Reproduction Number is bigger than one. In this particular case, the local stability of this endemic equilibrium was not explored yet.

Although it was possible to describe analytically the equilibrium with coexistence of two strains, in the symmetric case, the expression for the value of $S^{*}$ shows a complex dependency of the parameters. Also, it can be noticed that the characteristic equation of the system is a transcendental equation, with typically having infinitely many roots.

Thus, we are going to do a numerical analysis in order to understand the stability of the endemic equilibrium, analysing the sign of the roots of the characteristic equation, numerically, for some values of the parameter $\phi$, using it as a bifurcation parameter.

For all value of $\phi>0$, we already know that the coexistence equilibrium exists and, thus, we can numerically verify the value of $S^{*}$, for each value of $\phi$, then we calculate the roots of the characteristic equation, as we are going to show in the next figures. 


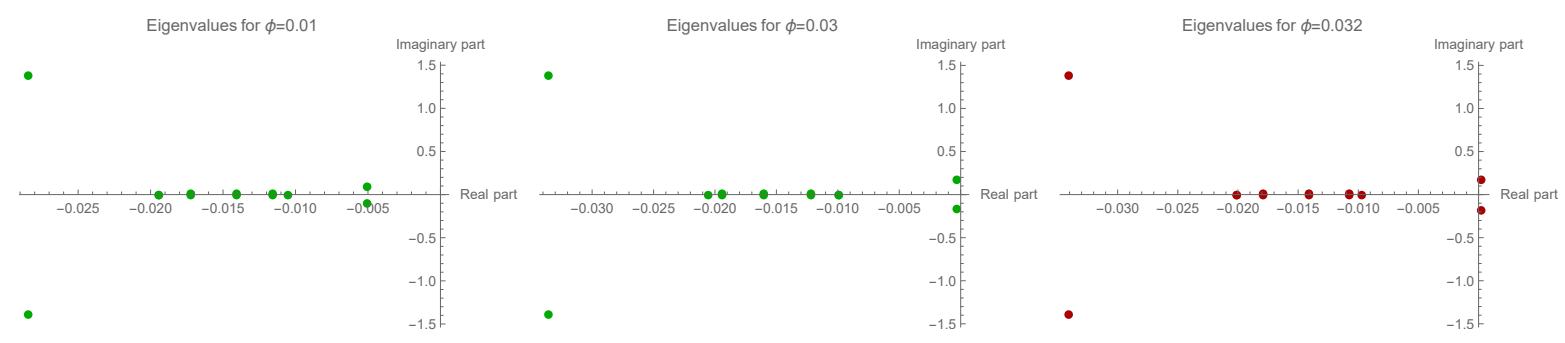
(a) $\phi=0.01$
(b) $\phi=0.03$
(c) $\phi=0.032$

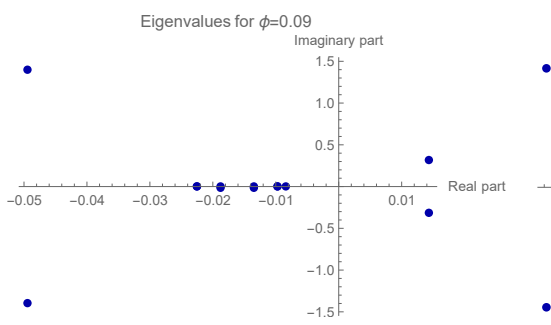

(d) $\phi=0.09$

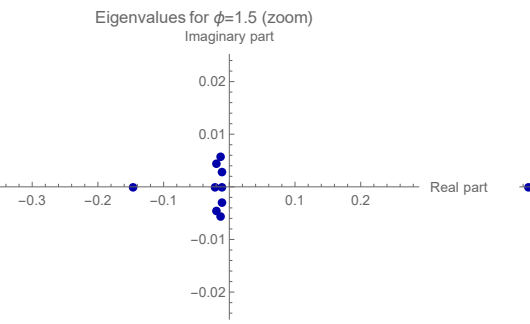

(g) $\phi=1.5$ zoom

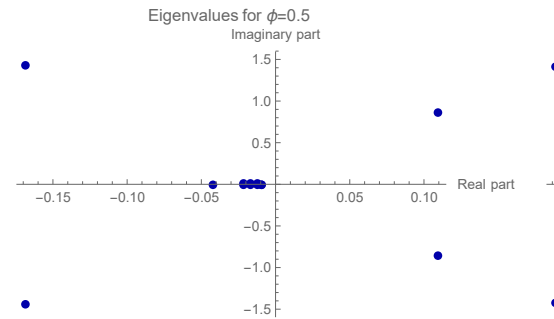

(e) $\phi=0.5$

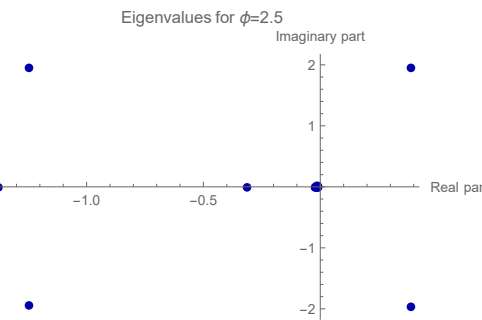

(h) $\phi=2.5$

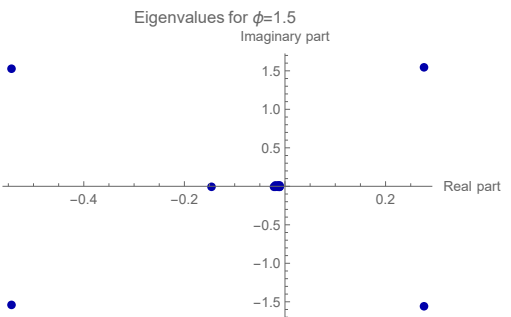

(f) $\phi=1.5$

Figure 3.1: The figures show the eigenvalues of the endemic equilibrium in the complex plane, for each value of $\phi$. The values used in the simulations are found on table $(3.2)$ with $\beta=$ $\beta_{1}=\beta_{2}=180$. Figures $3.1 \mathrm{a}$ ) to $3.1 \mathrm{~h}$ ) show that a purely imaginary eigenvalue appears for $\phi \approx 0.032$

As known, a Hopf bifurcation arises when looking for the eigenvalues of the Jacobian matrix of the continuous parametric dynamical system evaluated at a steady point of it, all eigenvalues have negative real part except one conjugate non-zero purely imaginary pair that cross the imaginary axis, because of a variation of the parameter.

Thus, analysing numerically the local stability of the endemic equilibrium $D_{3}$, figure (3.1c) shows that the matrix has a pair of conjugated complex eigenvalues, that change the sign of the real part as $\phi$ increases. Thus, a Hopf bifurcation occurs when the parameters $\phi$ is $\approx 0.032$ 


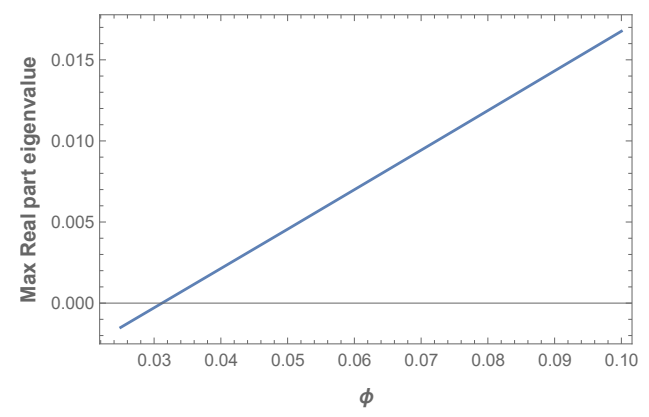

(a) $\phi \in(0,0.1)$

Figure 3.2: Maximum value of the real part of the eigenvalues of the endemic equilibrium.

To complete the analysis, figure 3.2a shows the maximum of the real part of eigenvalues for each value of parameter $\phi$. Figure (3.2a) shows that as $\phi$ approaches the value 0.032 , the biggest real part of the eigenvalues crosses the x-axis, when the Hopf bifurcation occurs and, it remains positive.

\subsubsection{Asymmetric case}

According to our analysis over chapter 3 , the coexistence equilibrium $D_{3}$ exists only if the maximum of the Reproduction number is bigger than one and, if the Invasion Reproduction number is bigger than one. In this case, the Boundary equilibrium loses stability and the coexistence equilibrium, with the coexistence of the two serotypes, rises and remains in the invariant region.

On figures above, we can see the parameters region for the stability of the Boundary equilibriums and, for the Disease Free equilibrium (DFE) as well as the parameters region for the existence of the Coexistence Endemic equilibrium. 


\section{Stability and existence region of the equilibriums}

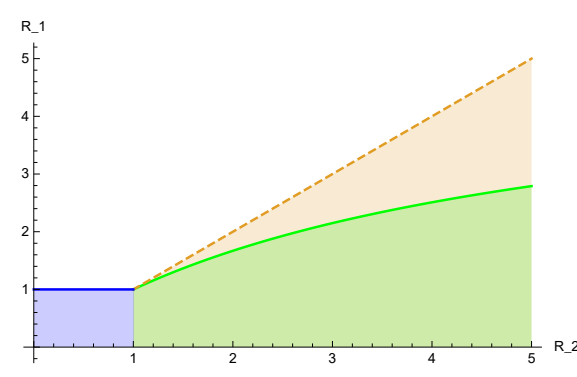

(a) $\phi=0.2$

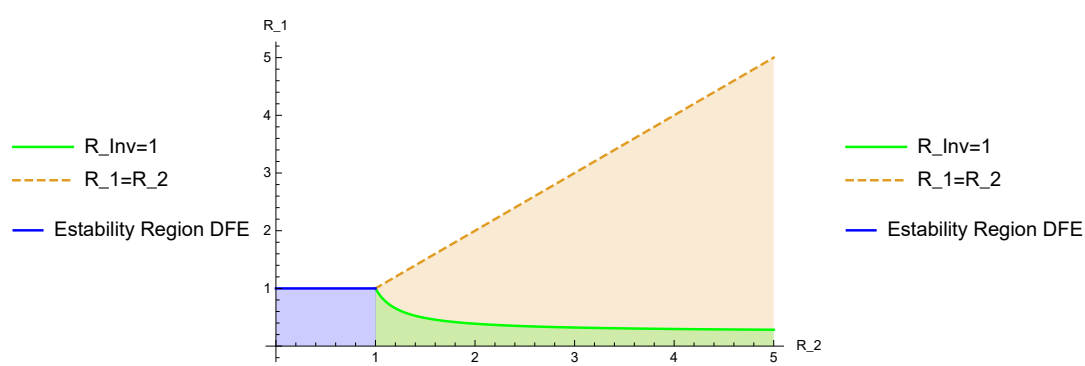

(b) $\phi=4.2$

Figure 3.3: The blue region represents the parameters region for which the DFE is globally stable $\left(\mathcal{R}_{0} \leq 1\right)$. The green region represents where the Boundary equilibrium is locally stable $\left(\mathcal{R}_{\text {Inv }} \leq 1\right)$. The coral one represents the existence region of the Coexistence Endemic equilibrium $\left(\mathcal{R}_{\text {Inv }}>1\right)$. Figure $(3.3 \mathrm{a})$ with $\phi=0.2$ and figure $(3.3 \mathrm{~b})$ with $\phi=4.2$.

Figures (3.3a) and (3.3b) show that, as the value $\phi$ increases, the parameters region of the stability for the Boundary equilibrium decreases, forcing the endemic equilibrium coexist within the region.

According to the figures (3.3a) and $3.3 \mathrm{~b}$ ) there is a threshold for the value of $\phi$, in each case, which satisfies $\mathcal{R}_{\text {Inv }}>1$. Then, starting for this threshold, the Endemic equilibrium is in the region and it makes sense to look for the eigenvalues. Thus, we can numerically verify the local dynamic near the correspondent endemic equilibrium for each value of $\phi$.

In the asymmetric case, we divide in two cases:

Case (i): $\mathcal{R}_{0}>1, \mathcal{R}_{1}<1$

In this case, with values for the parameters on table (3.2) with $\beta_{1}=45$ and $\beta_{2}=180$, which give $\mathcal{R}_{1}=0.87$, and $\mathcal{R}_{0}=3.46$, the $\mathcal{R}_{\text {Inv }}$ is bigger than one for $\phi=1.23$. Then, for all value of $\phi>1.23$ we have the existence of the endemic equilibrium, and the correspondents eigenvalues, as shown in the figures. 


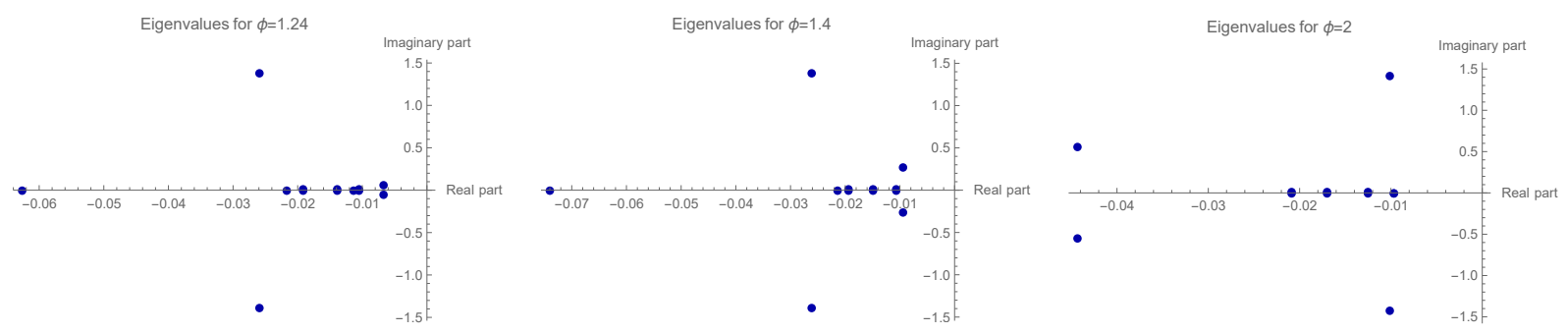
(a) $\phi=1.24$
(b) $\phi=1.4$
(c) $\phi=2$

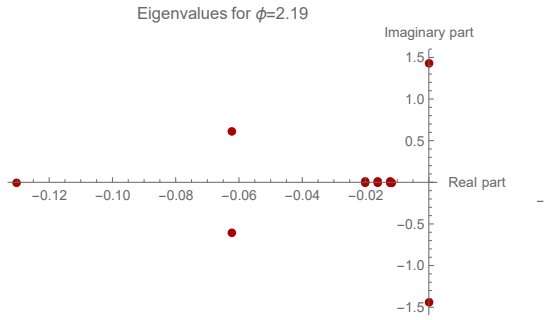

(d) $\phi=2.19$

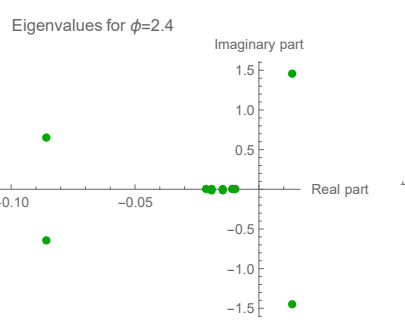

(e) $\phi=2.4$

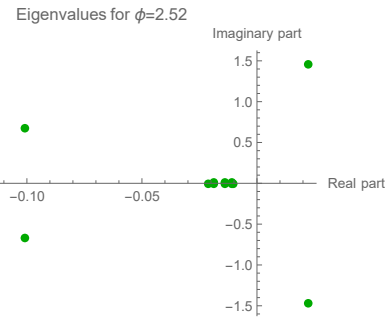

(f) $\phi=2.52$

Figure 3.4: The figures show the eigenvalues of the endemic equilibrium in the complex plane, for each value of $\phi$. The values used in the simulations are found on table $(3.2)$ with $\beta_{1}=45$ and $\beta_{2}=180$. Figure $(3.4 \mathrm{~d})$ shows that a purely imaginary eigenvalue appears for $\phi \approx 2.19$.

Thus, analysing numerically the local stability of the endemic equilibrium $D_{3}$, figure (3.4a) to (3.4f) show that the characteristic equation has a pair of conjugated complex roots, which changes the sign of the real part as $\phi$ increases. Thus, a Hopf bifurcation occurs when the parameter $\phi \approx 2.19$.

Case (ii): $\mathcal{R}_{0}>1, \mathcal{R}_{1}>1$

In this case, with values for the parameters on table (3.2) with $\beta_{1}=120$ and $\beta_{2}=180$, which give $\mathcal{R}_{1}=2.31$, and $\mathcal{R}_{0}=3.46$, the $\mathcal{R}_{\text {Inv }}$ is bigger than one for $\phi=0.205$. Then, for all value of $\phi>0.205$ we have the existence of the endemic equilibrium and the correspondents eigenvalues, as shown on the figures. 


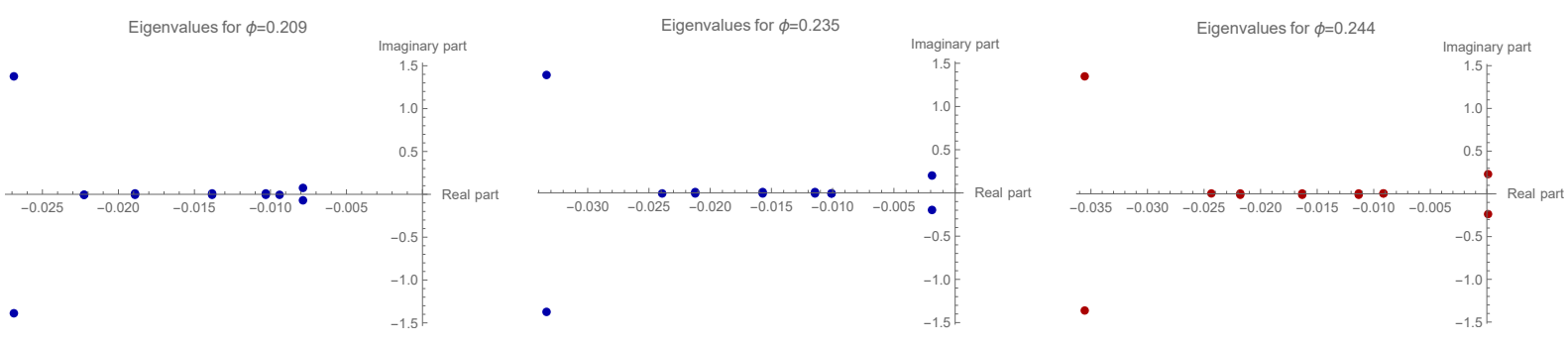
(a) $\phi=0.209$
(b) $\phi=0.235$
(c) $\phi=0.244$

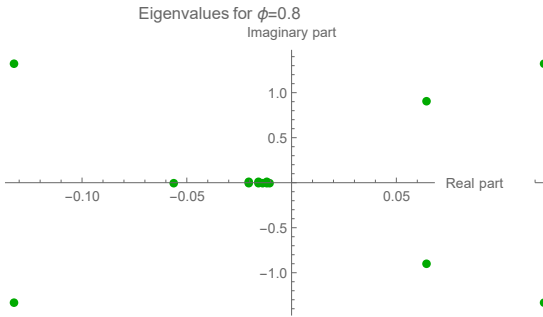

(d) $\phi=0.8$

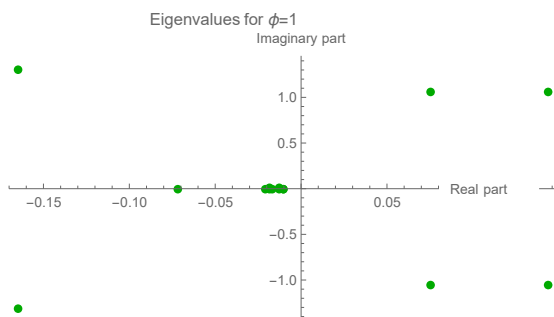

(e) $\phi=1$

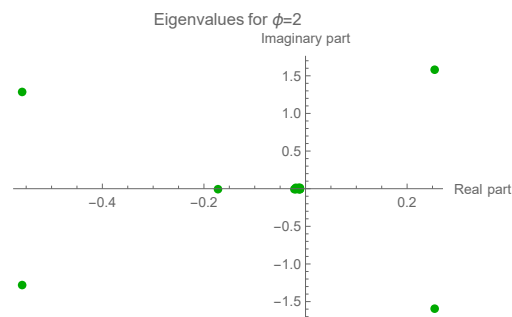

(f) $\phi=2$

Figure 3.5: The figures show the eigenvalues of the endemic equilibrium in the complex plane, for each value of $\phi$. The values used in the simulations are found on table (3.2) with $\beta_{1}=120$ and $\beta_{2}=180$. Figure $(3.5 \mathrm{c})$ shows that a purely imaginary eigenvalue appears for $\phi \approx 0.244$.

Thus, analysing numerically the local stability of the endemic equilibrium $D_{3}$, figure (3.5a) to 3.5f show that the characteristic equation has a pair of conjugated complex roots, which changes the sign of the real part as $\phi$ increases. Thus, a Hopf bifurcation occurs when the parameter $\phi \approx 0.244$.

\subsubsection{Bifurcation Structure}

Through the figures, we have showed numerically in the previous section that the Endemic coexistence equilibrium changes the stability as the parameter $\phi$ changes. As $\phi$ increases from small values through critical value, $\phi_{c}$, the steady state changes from a stable focus to an unstable steady state. Therefore, Hopf bifurcation occurs and, consequently, we conclude that closed periodic orbit will be found in a small neighbourhood of $\phi_{c}$.

In order to see the limit cycle around the equilibrium, in a small vicinity of the critical value, bifurcation diagrams are shown on the next figures. 


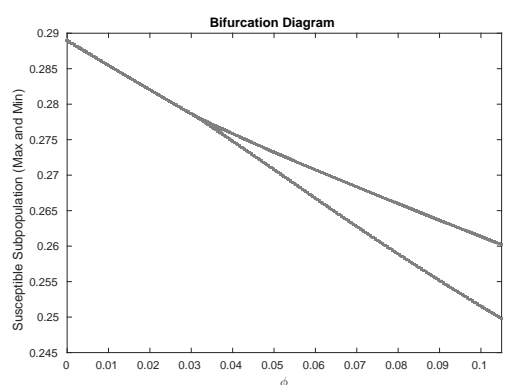

(a) Symmetric case $\left(\phi_{c}=0.032\right)$

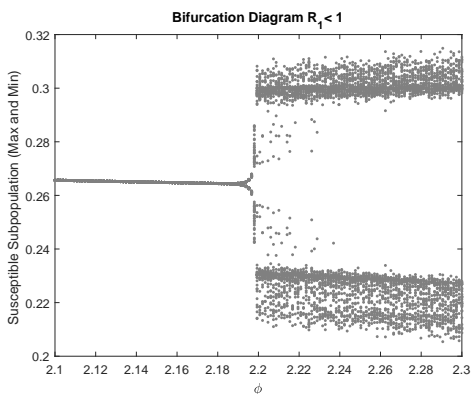

(b) Asymmetric case: $\mathcal{R}_{1}=0.87\left(\phi_{c}=2.19\right)$

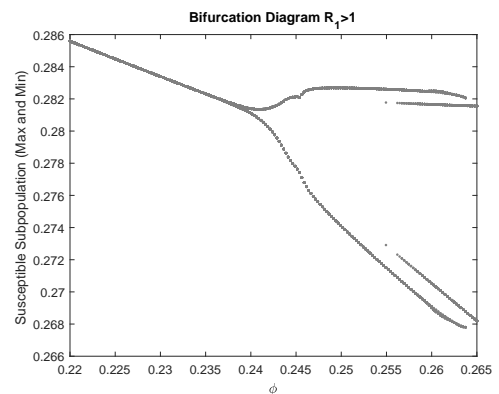

(c) Asymmetric case: $\mathcal{R}_{1}=2.31\left(\phi_{c}=0.244\right)$

Figure 3.6: Bifurcation diagrams for symmetric and asymmetric cases. In the horizontal axis, the parameter $\phi$ varies in a vicinity of $\phi_{c}$, while in the vertical axis, the maximum and minimum values for susceptible population are plotted.

In the symmetric case, the Hopf bifurcation occurs at $\phi_{c}=0.032$, it means that the solutions exhibit a small amplitude limit cycle around the endemic equilibrium. A stable limit cycle clearly arises and goes away from the equilibrium (figure (3.6a) ). Also, the amplitude of the limit cycle increases gradually as the parameter $\phi$ moves away from the critical bifurcation point. Thus, it is possible to conclude that a supercritical Hopf bifurcation occurred.

In the asymmetric case, the Hopf bifurcation occurs at $\phi_{c}=2.19$ and $\phi_{c}=0.244$, it means that the solutions exhibit a small amplitude limit cycle around the endemic equilibrium. A stable limit cycle arises close to the critical Bifurcation point and goes away from the unstable equilibrium (figure (3.6b) ) and (figure (3.6c)). Thus, it is possible to conclude that a supercritical Hopf bifurcation has occurred.

Over the next section, solutions will be plotted for different values of $\phi$, in order to support the theoretical analysis (about stability of the equilibriums), as well as verifying the local numerical analysis (about the H. B.), also showing the asymptotic behaviour for parameter values further from bifurcation value.

\subsubsection{Solutions of the system}

In this section, we are going to explore and to analyse numerically the solutions of the system in order to understand the model better, as well as using this numerical approach to obtain information about the solutions of the system for values of parameter $\phi$ further from the bifurcation value. 


\section{Symmetric case}

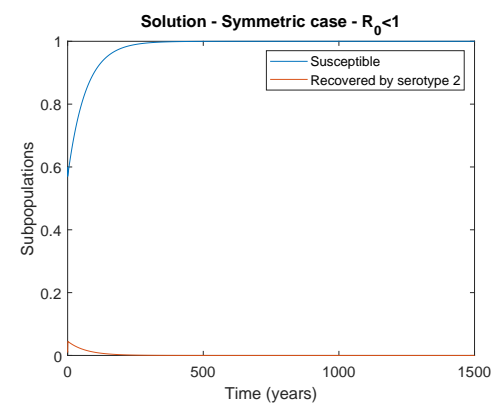

(a) $\phi=1$

Figure 3.7: Solutions of the system for $\phi=1, \beta=45, \mathcal{R}_{0}=0.87<1$.Initial conditions: $(0.6,0.01,0.02,0,0,0,0.01,0,0)$.

The figure (3.7a) shows the solutions of the system at the symmetric case for the parameter values on the table $(3.2)$ with $\phi=1, \beta=45$ giving $\mathcal{R}_{0}=0.87<1$. In this case, the long term behaviour of the solutions tends to the DFE.

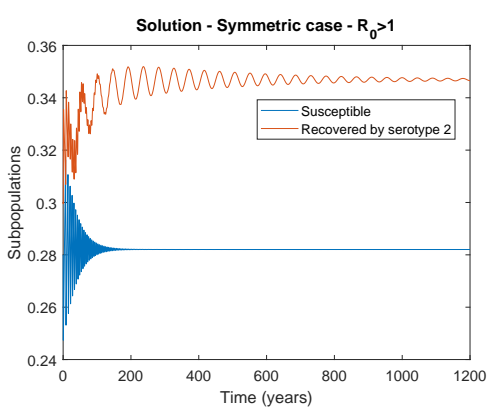

(a) $\phi=0.02$

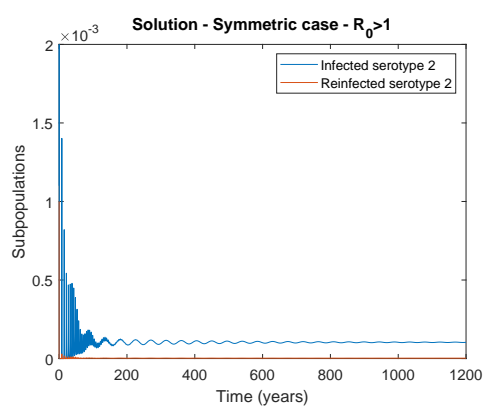

(b) $\phi=0.02$

Figure 3.8: Solutions of the system for $\phi=0.02, \beta=180, \mathcal{R}_{0}=3.46>1$ Initial conditions $(0.28,0.00011,0.0011,0.002,0.002,0.3,0.3,0.001,0.001)$.

The figure (3.8a) and (3.8b) show the solutions of the system at the symmetric case for the parameter values on the table $(3.2)$ with $\phi=0.02, \beta=180$ giving $\mathcal{R}_{0}=3.46>1$. In this case, the long term behaviour of the solutions tends to the coexistence (endemic) equilibrium. This happens for all values of $\phi \in(0,0.032)$. 


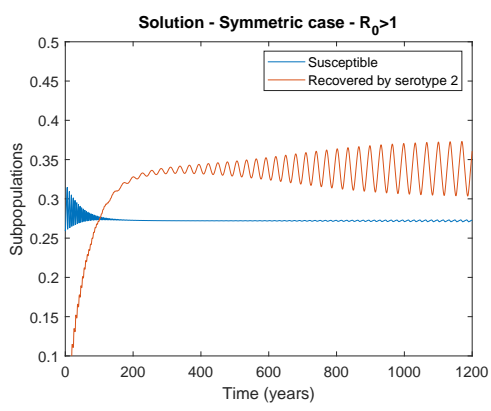

(a) $\phi=0.05$

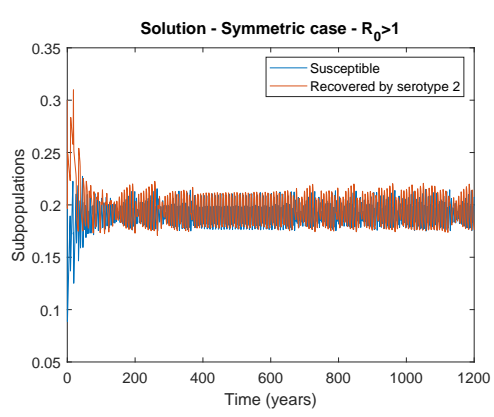

(d) $\phi=0.5$

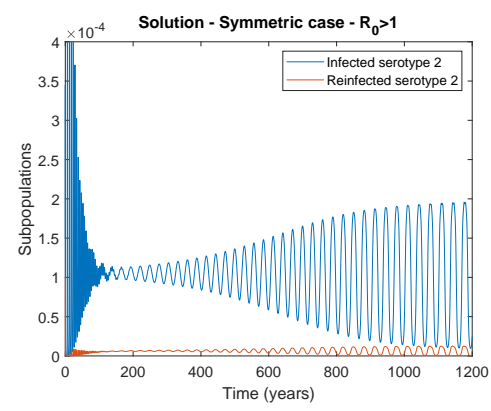

(b) $\phi=0.05$

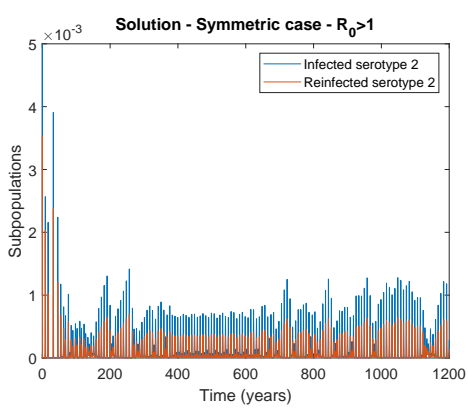

(e) $\phi=0.5$

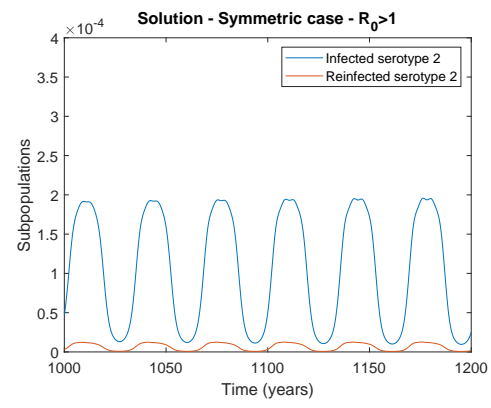

(c) $\phi=0.05$

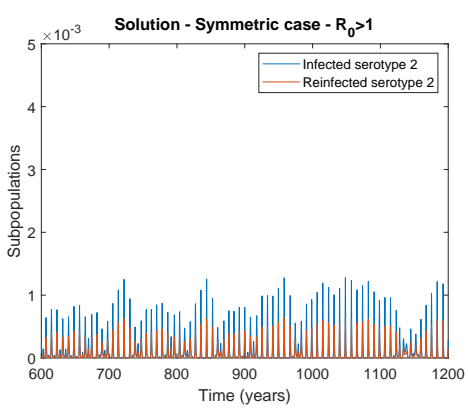

(f) $\phi=0.5$

Figure 3.9: Solutions of the system for $\beta=180, \mathcal{R}_{0}=3.46>1$. For $\phi=0.05$, initial conditions $(0.26,0.00011,0.00015,0.034,0.036,0.00010,0.00012)$. For $\phi=0.5$, initial conditions $(0.3,0.0011,0.002,0.2,0.3,0.001,0.002)$.

The figure (3.9a) to 3.9c) show the solutions of the system at the symmetric case for the parameter values on the table $(3.2)$ with $\phi=0.05, \beta=180$ giving $\mathcal{R}_{0}=3.46>1$. And figures (3.9d) and (3.9f), for $\phi=0.5$. In the first case, the equilibrium is unstable and $\phi$ is taken close to the Hopf bifurcation value, consequently, closed periodic orbit will be found. The solutions indicate that a limit cycle appears and it is stable for small values of $\phi$ close to $\phi_{c}=0.032$.

As $\phi$ increases far from the bifurcation critical value, changes in the behaviour of the solutions are able to be seen ( figures (3.9d) and (3.9e)). In this case, for long term behaviour, it is possible to see different oscillations and a complex behaviour (3.9f). 


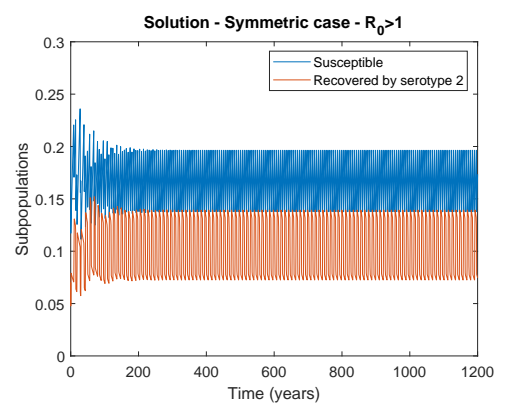

(a) $\phi=1.2$

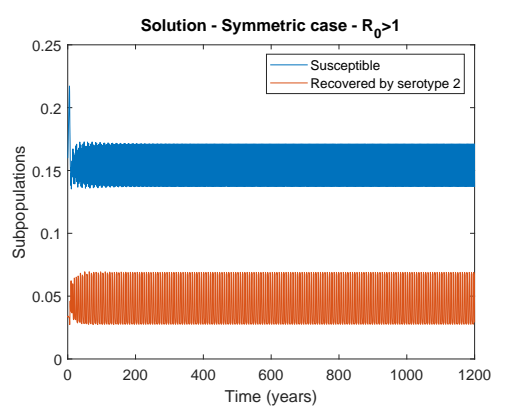

(d) $\phi=3$

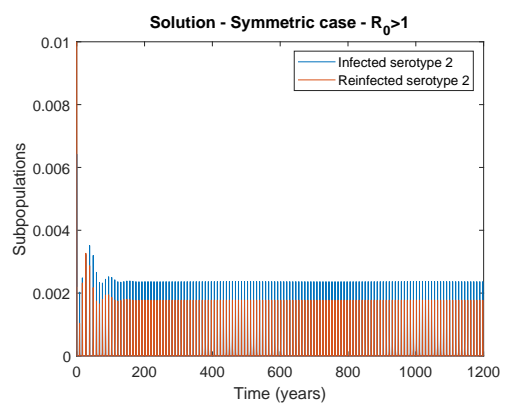

(b) $\phi=1.2$

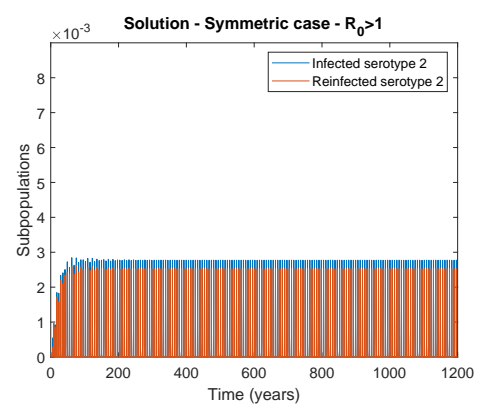

(e) $\phi=3$

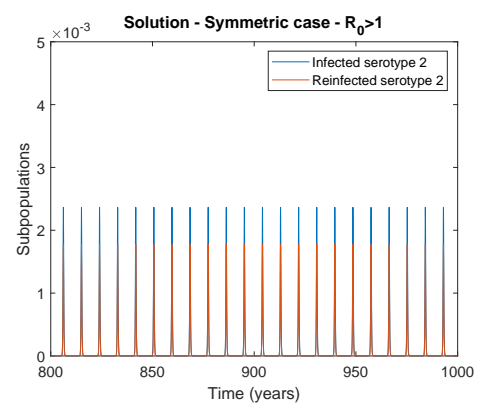

(c) $\phi=1.2$

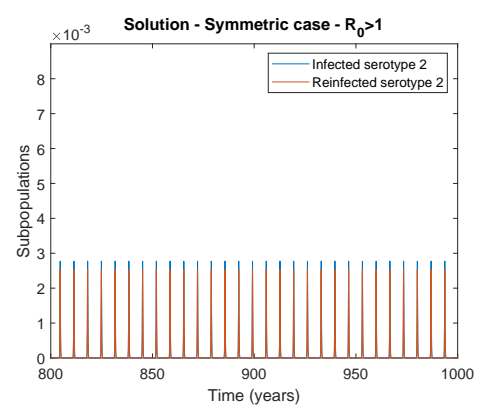

(f) $\phi=3$

Figure 3.10: Solutions of the system for $\phi=3, \beta=180$. For $\phi=1.2$, initial condition $(0.2,0.0011,0.0022,0.01,0.02,0.07,0.07,0.01,0.02)$. For $\phi=3$, initial condition $(0.16,0.00011,0.00013,0.003,0.003,0.034,0.034,0.00012,0.0001)$.

The figure (3.10a) to $3.10 \mathrm{c})$ show the solutions of the system at the symmetric case for the parameter values on the table 3.2 with $\beta=180$ giving $\mathcal{R}_{0}=3.46>1$. In both cases, $\phi=1.2$ and $\phi=3$, the solutions of the system converge to periodic orbits. 


\section{Asymmetric case}

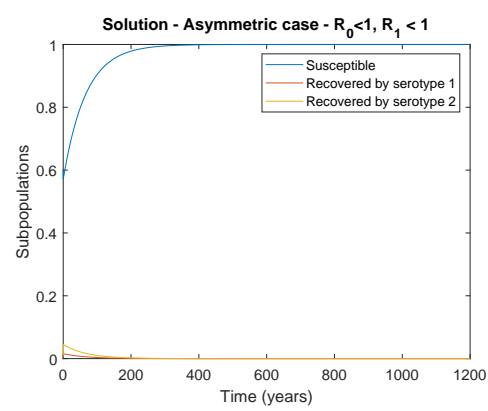

(a) $\phi=1$

Figure 3.11: Solutions of the system for $\phi=1, \beta_{1}=40, \mathcal{R}_{1}=0.76<1, \beta_{2}=45, \mathcal{R}_{2}=$ $0.87<1$. Initial conditions: $(0.6,0.01,0.02,0,0,0,0.01,0,0)$.

The figure (3.11a) shows the solutions of the system at the asymmetric case for the parameter values on the table 3.2 with $\phi=1, \beta_{1}=40, \mathcal{R}_{1}=0.76<1, \beta_{2}=45, \mathcal{R}_{2}=$ $0.87<1$. In this case, the long term behaviour of the solutions tends to the DFE.

Now, we are going to explore and to analyse numerically the solutions of the system, in the asymmetric case, when $\beta_{1}<\beta_{2}$ and $\mathcal{R}_{1}<1<\mathcal{R}_{2}$.

Case (i): $\mathcal{R}_{0}>1, \mathcal{R}_{1}<1$

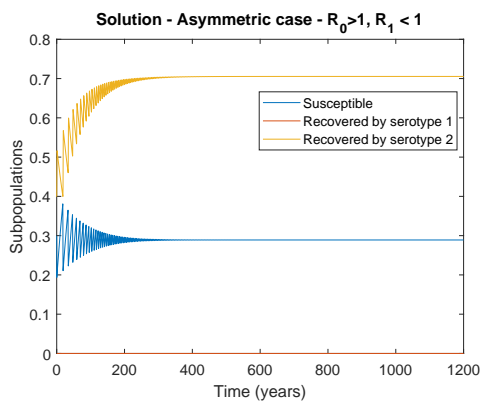

(a) $\phi=0.5$

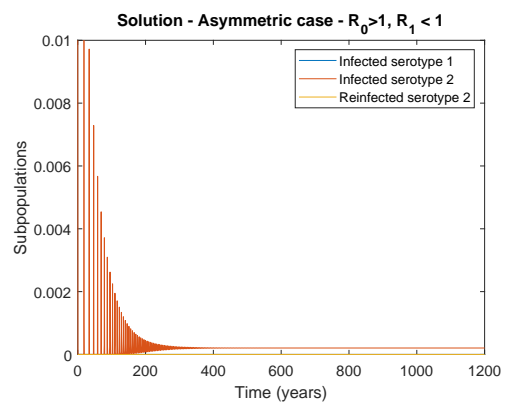

(b) $\phi=0.5$

Figure 3.12: Solutions of the system for $\phi=0.5, \beta_{1}=45, \mathcal{R}_{1}=0.87<1, \beta_{2}=180$, $\mathcal{R}_{2}=3.46>1$. Initial conditions: $(0.3,0.000001,0.02,0,0.005,0,0.4,0,0)$.

The figures (3.12a) and (3.12b) show the solutions of the system at the asymmetric case for the parameter values on the table (3.2) with $\phi=0.5, \beta_{1}=45$ giving $\mathcal{R}_{1}=0.87<1$ and, $\beta_{2}=180$ giving $\mathcal{R}_{2}=3.46>1$. In this case, the long term behaviour of the solutions tends to the Boundary equilibrium $D_{2}$, it means that the Boundary equilibrium is locally asymptotically 
stable. In addition, the coexistence equilibrium is not in the positive region. This happens for all values of $\phi \in(0,1.23)$.

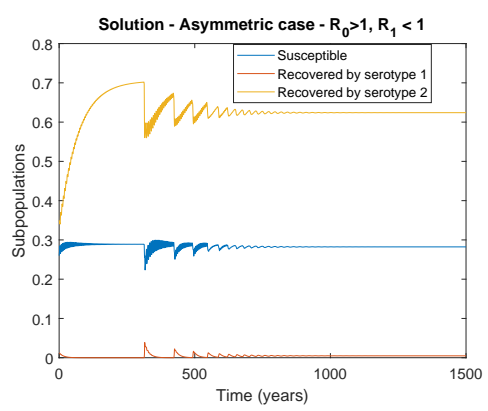

(a) $\phi=1.4$

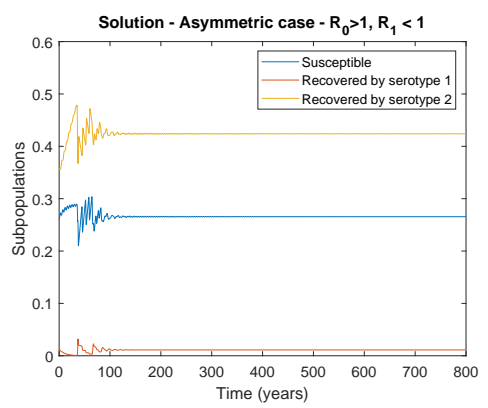

(c) $\phi=2.1$

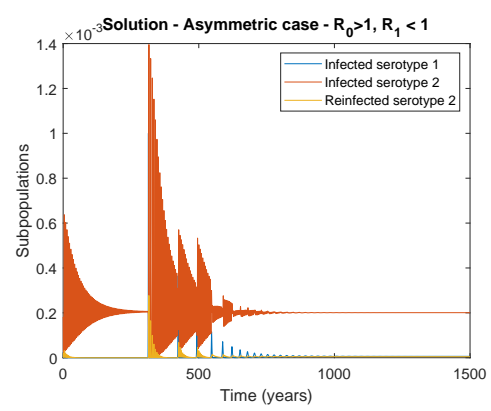

(b) $\phi=1.4$

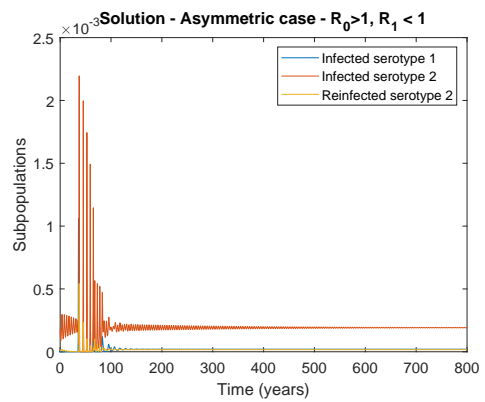

(d) $\phi=2.1$

Figure 3.13: Solutions of the system for $\beta_{1}=45, \mathcal{R}_{1}=0.87<1$, $\beta_{2}=180, \mathcal{R}_{2}=3.46>1 . \quad$ For $\phi=1.4$, initial conditions $(0.26,0.000024,0.00018,0.00063,0.0048,0.012,0.35,0.000021,0.000085) . \quad$ For $\phi=2.1$, initial conditions $(0.2601,0.0000244,0.00018,0.00063,0.0048,0.0115,0.358,0.0000209,0.000084)$.

The figures (3.13a and (3.13b) show the solutions of the system at the asymmetric case for the parameter values on the table $(3.2)$ for $\phi=1.4, \beta_{1}=45$ giving $\mathcal{R}_{1}=0.87<1$ and, $\beta_{2}=180$ giving $\mathcal{R}_{2}=3.46>1$. And, for $\phi=2.1$, the solutions are shown by figures (3.13c) and (3.13d). In both cases, the long term behaviour of the solutions converges to the Coexistence equilibrium, it means that the Coexistence equilibrium is locally asymptotically stable. This happens for all values of $\phi \in(1.23,2.2)$. 


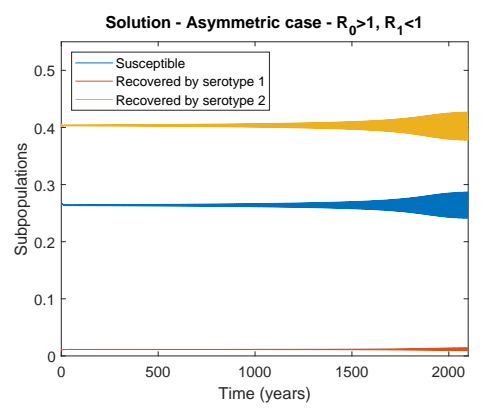

(a) $\phi=2.21$

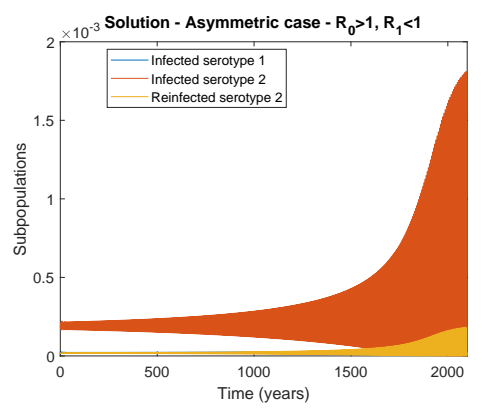

(b) $\phi=2.21$

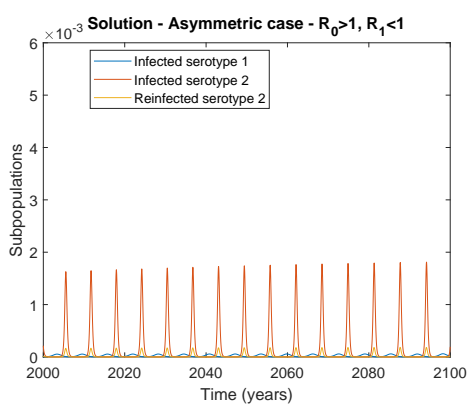

(c) $\phi=2.21$

Figure 3.14: Solutions of the system for $\phi=2.21, \quad \beta_{1}=45$, $\mathcal{R}_{1}=0.87<1, \beta_{2}=180, \mathcal{R}_{2}=3.46>1 . \quad$ Initial conditions: $(0.2643,0.00002122,0.000190,0.01123,0.4070,0.00001776,0.0000715)$.

The figures $3.14 \mathrm{a})$ to $3.14 \mathrm{c})$ show the solutions of the system at the asymmetric case for the parameter values on the table (3.2) for $\phi=2.21, \beta_{1}=45$ giving $\mathcal{R}_{1}=0.87<1$ and, $\beta_{2}=180$ giving $\mathcal{R}_{2}=3.46>1$. In this case, the solution for long term behaviour exhibits a limit cycle.

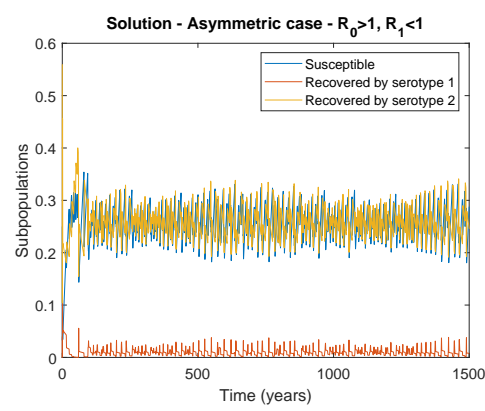

(a) $\phi=3.5$

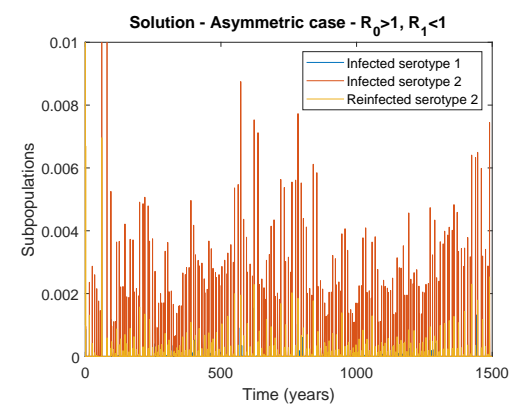

(b) $\phi=3.5$

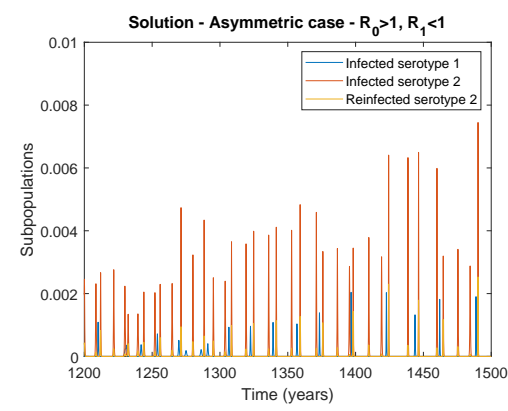

(c) $\phi=3.5$

Figure 3.15: Solutions of the system for $\phi=3.5, \beta_{1}=45, \mathcal{R}_{1}=0.87<1, \beta_{2}=180$, $\mathcal{R}_{2}=3.46>1$. Initial conditions: $(0.25,0.08,0.2,0.05,0.15,0.04,0.56,0.001,0.009)$.

The figures (3.15a) to (3.15c) show the solutions of the system at the asymmetric case for the parameter values on the table (3.2) with $\phi=3.5, \beta_{1}=45$ giving $\mathcal{R}_{1}=0.87<1$ and, $\beta_{2}=180$ giving $\mathcal{R}_{2}=3.46>1$. In this case, the solutions show a complex behaviour.

Now, we are going to explore and to analyse numerically the solutions of the system in the asymmetric case, when $\beta_{1}<\beta_{2}$ and $1<\mathcal{R}_{1}<\mathcal{R}_{2}$.

Case (ii): $\mathcal{R}_{0}>1, \mathcal{R}_{1}>1$ 


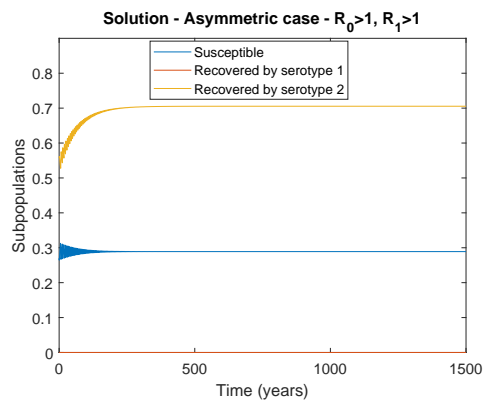

(a) $\phi=0.1$

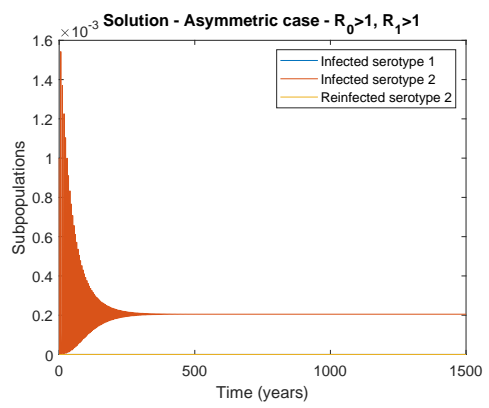

(b) $\phi=0.1$

Figure 3.16: Solutions of the system for $\phi=0.1, \beta_{1}=120, \mathcal{R}_{1}=2.31>1, \beta_{2}=180$, $\mathcal{R}_{2}=3.46>1$. Initial conditions: $(0.27,0.000001,0.001,0.00001,0.05,0,0.56,0,0)$.

The figures (3.16a) and (3.16b) show the solutions of the system at the asymmetric case for the parameter values on the table (3.2) with $\phi=0.1, \beta_{1}=120$ giving $\mathcal{R}_{1}=2.31>1$ and, $\beta_{2}=180$ giving $\mathcal{R}_{2}=3.46>1$. In this case, the long term behaviour of the solutions tends to the Boundary equilibrium $D_{2}$, it means that the Boundary equilibrium is locally asymptotically stable. In addition, the Coexistence equilibrium is not in the positive region. This happens for all values of $\phi \in(0,0.205)$.

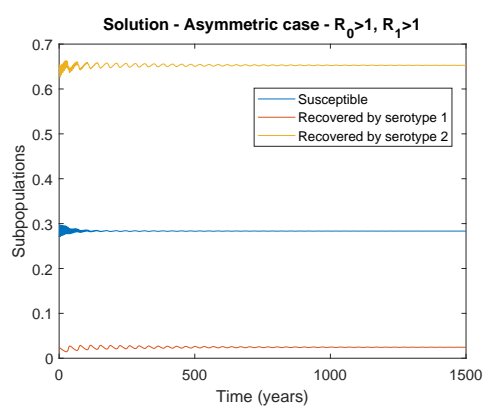

(a) $\phi=0.23$

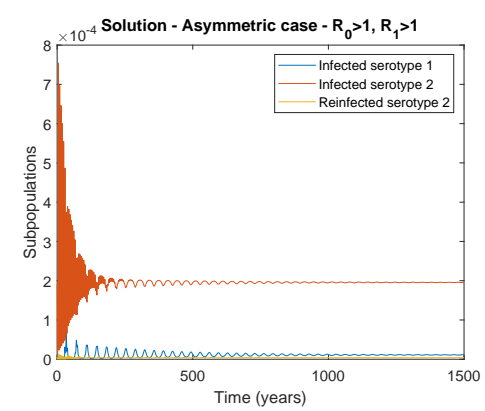

(b) $\phi=0.23$

Figure 3.17: Solutions of the system for $\beta_{1}=120, \mathcal{R}_{1}=$ $2.31>1, \quad \beta_{2}=180, \quad \mathcal{R}_{2}=3.46>1 . \quad$ Initial conditions: $(0.27,0.000011,0.00019,0.00027,0.00505,0.0237,0.6546,0.0000031,0.0000011)$.

The figures (3.17a) and (3.17b) show the solutions of the system at the asymmetric case for the parameter values on the table (3.2) with $\phi=0.23, \beta_{1}=120$ giving $\mathcal{R}_{1}=2.31>1$ and, $\beta_{2}=180$ giving $\mathcal{R}_{2}=3.46>1$. In this case, the long term behaviour of the solutions tends to the Coexistence equilibrium, it means that the Coexistence equilibrium is locally asymptotically 
stable. This happens for all values of $\phi \in(0.205,0.244)$.

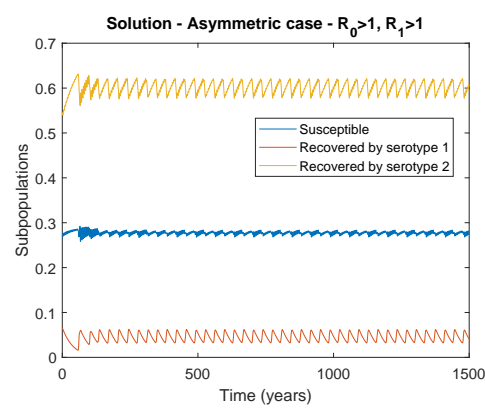

(a) $\phi=0.26$

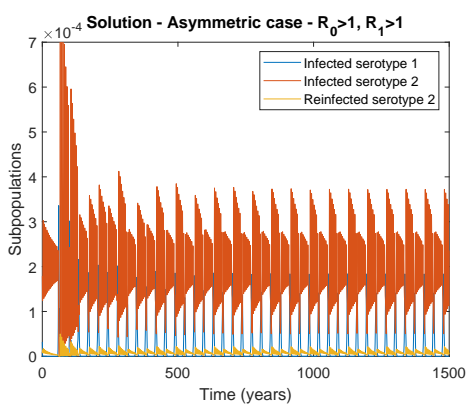

(b) $\phi=0.26$

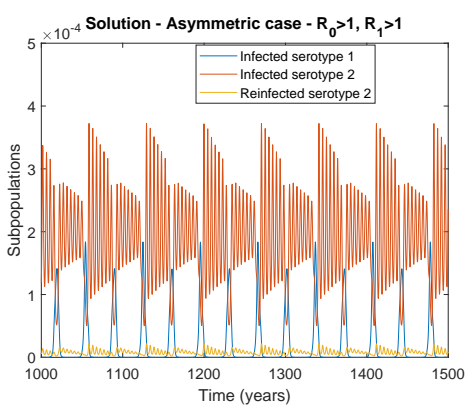

(c) $\phi=0.26$

Figure 3.18: Solutions of the system for $\phi=0.26, \quad \beta_{1}=120$, $\mathcal{R}_{1}=2.31>1, \beta_{2}=180, \mathcal{R}_{2}=3.46>1 . \quad$ Initial conditions: $(0.269,0.0000321,0.0001786,0.000828,0.00461,0.0653,0.546,0.0000129,0.0000195)$.

The figures (3.18a) to (3.18c) show the solutions of the system at the asymmetric case for the parameter values on the table (3.2) with $\phi=0.26, \beta_{1}=120$ giving $\mathcal{R}_{1}=2.31>1$ and, $\beta_{2}=180$ giving $\mathcal{R}_{2}=3.46>1$. In this case, a stable limit cycle appears and the solutions converge to a periodic orbit.

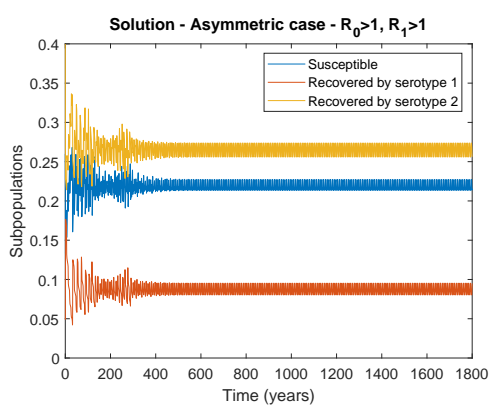

(a) $\phi=0.8$

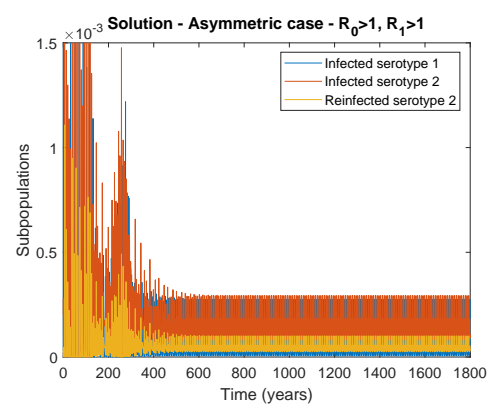

(b) $\phi=0.8$

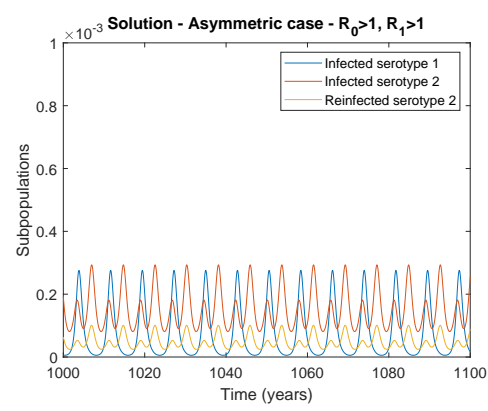

(c) $\phi=0.8$

Figure 3.19: Solutions of the system for $\phi=0.8, \beta_{1}=120, \mathcal{R}_{1}=2.31>1, \beta_{2}=180, \mathcal{R}_{2}=$ $3.46>1$. Initial conditions: $(0.26,0.000032,0.0002,0.0009,0.005,0.05,0.4,0.00001,0.00002)$.

The figures (3.19a) to (3.19c) show the solutions of the system at the asymmetric case for the parameter values on the table (3.2) with $\phi=0.8, \beta_{1}=120$ giving $\mathcal{R}_{1}=2.31>1$ and, $\beta_{2}=180$ giving $\mathcal{R}_{2}=3.46>1$. In this case, a stable limit cycle appears and the solutions converge to a periodic orbit. 


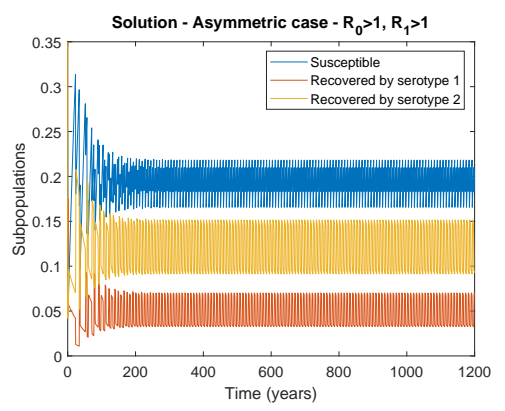

(a) $\phi=2$

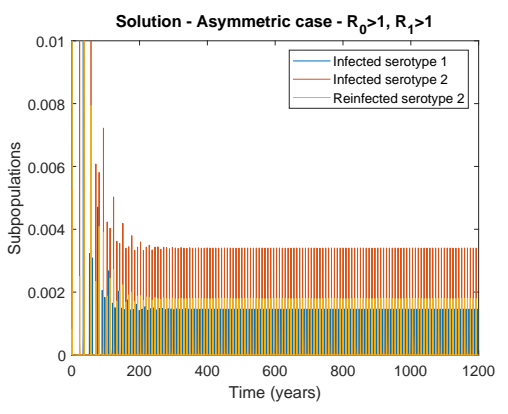

(b) $\phi=2$

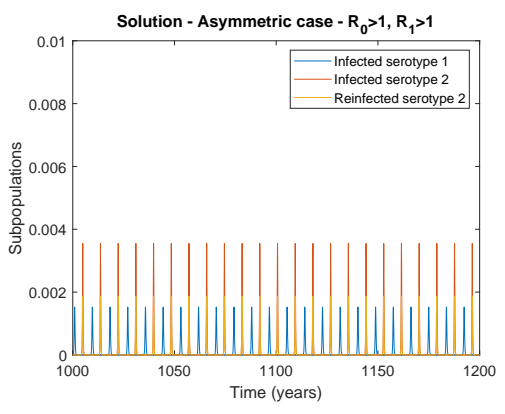

(c) $\phi=2$

Figure 3.20: Solutions of the system for $\phi=2, \beta_{1}=120, \mathcal{R}_{1}=2.31>1, \beta_{2}=180, \mathcal{R}_{2}=$ $3.46>1$. Initial conditions: $(0.25,0.000042,0.0009,0.001,0.01,0.05,0.35,0.00002,0.00003)$.

The figures (3.20a) to 3.20c) show the solutions of the system at the asymmetric case for the parameter values on the table (3.2) with $\phi=2, \beta_{1}=120$ giving $\mathcal{R}_{1}=2.31>1$ and, $\beta_{2}=180$ giving $\mathcal{R}_{2}=3.46>1$. In this case, a stable limit cycle appears and the solutions converge to a periodic orbit.

Although we conclude mathematically that the solution of the system goes to an equilibrium or goes to a periodic orbit, this happens for a long time behaviour. It means, it takes much time to get in the asymptotic behaviour. Therefore, biologically speaking, it is hard to predict the next episode of the disease for a short period of time.

\subsection{Main Results}

We can summarize our main results on the table below, where local and global stability of the DFE and the boundary equilibriums were theoretically proved. While, the local stability and bifurcations of the Coexistence Endemic were numerically showed. 
Table 3.3: Stability of the solutions of the delay system: Symmetric parameters

\begin{tabular}{lllll}
\hline Basic R. Number & DFE & Eq. $D_{1}$ & Eq. $D_{2}$ & Int. Equilibrium $D_{3}$ \\
\hline $\mathcal{R}_{1}=\mathcal{R}_{2}$ & & & & \\
\hline $\mathcal{R}_{0} \leq 1$ & GAS & No & No & No \\
$\mathcal{R}_{0}>1$ & Unstable & Unstable & Unstable & LAS for $\phi<\phi_{c}$ \\
& & & & H. B. (stable limit cycle) \\
& & & & Unstable $\phi>\phi_{c}$ \\
\hline
\end{tabular}

Table 3.4: Stability of the solutions of the delay system: Asymmetric parameters

\begin{tabular}{llllll}
\hline Basic R. N. & Invasion N. & DEF & Eq. $D_{1}$ & Eq. $D_{2}$ & Int. Eq. $D_{3}$ \\
\hline $\mathcal{R}_{1}<\mathcal{R}_{2}$ & $\mathcal{R}_{\text {Inv }}$ & & & & \\
\hline $\mathcal{R}_{0} \leq 1$ & $\mathcal{R}_{\text {Inv }}<1$ & GAS & No & No & No \\
$\mathcal{R}_{0}>1$ & $\mathcal{R}_{\text {Inv }}<1$ & Unstable & No $\left(\mathcal{R}_{1}<1\right)$ & GAS & No \\
& & & Unstable $\left(\mathcal{R}_{1}>1\right)$ & & \\
$\mathcal{R}_{0}>1$ & $\mathcal{R}_{\text {Inv }}>1$ & Unstable & Unstable & Unstable & LAS for $\phi<\phi_{c}$ \\
& & & & & H B (stable limit cycle) \\
& & & & & Unstable $\phi>\phi_{c}$ \\
\hline
\end{tabular}


CHAPTER 4

\section{Analysis of the Coexistence Endemic Equilibrium}

\subsection{Stability Analysis of the Endemic Equilibrium of the Time Delay System}

The analysis of the local stability of the endemic equilibrium (coexistence equilibrium) using the theory of the linearisation and the results in Brauer (1978) was not successful. We will have to deal with transcendental characteristic equation, as well as the fact it was not possible to describe the endemic equilibrium in terms of the parameters.

Therefore, in order to analyse the stability, we first analyse it numerically (chapter 3). We have found that a Hopf bifurcation occurs for a critical value of the parameter $\phi$ leading to periodic oscillations. The steady state was computed numerically for arbitrary $\phi$ and, as $\phi$ increases, the trajectories change from stable endemic coexistence equilibrium to complicated oscillatory dynamics.

Once the numerical results are in mind, in order to analyse analytically the bifurcation structure, we are going to use perturbation theory and symmetry in order to dribble the complexity of a direct calculation of the endemic equilibrium and the eigenvalues.

We are going to analyse now the general case for the time delay system. In order to do that, we are first going to use the idea by Domoshnitsky (2002). Domoshnitsky (2002) proposed a method which reduces a class of IDE to corresponding ODE system. If the elements of the kernel function in the integral are constants matrices or have the form

$$
(t-s)^{k} e^{-A(t-s)} \sin (B(t-s))
$$


where $k$ is an integer non-negative number, $A, B$ real numbers, then a new autonomous system can be written. Generalization of this method is proved by Domoshnitsky (2002) by using Cauchy functions and use kernel functions for the construction of the Cauchy matrix of an auxiliary ODE system.

Our purpose here is to prove analytically that in fact, also for the time delay system, the endemic equilibrium is unstable after the bifurcation value for the parameter $\phi$. Thus, we will choose for the kernel function $P(s)$, two functions of the form (4.1) and show these two cases. Generalizations can be done using the results of the Domoshnitsky (2002). For our main purpose these two cases are sufficient, although generalizations can be done in future research.

We are going to consider two particular cases:

Case (i): $P(s)=\cos (A s) e^{-\omega s}, A>0$.

We are going to analyse the case when $P(s)=\cos (A s) e^{-\omega s}$. Of course this function satisfies the necessary assumptions, such as, $P(0)=1, P(\infty)=0$ and $\int_{0}^{\infty} e^{-d s} P(s) d s<\infty$. Then,

$$
C_{i}(t)=\int_{0}^{t} \gamma I_{i}(s) \cos (A(t-s)) e^{-(\omega+d)(t-s)} d s
$$

and,

$$
C_{i}^{\prime}(t)=\gamma I_{i}(t)-(d+\omega) C_{i}(t)-A \int_{0}^{t} \gamma I_{i}(s) \operatorname{sen}(A(t-s)) e^{-(\omega+d)(t-s)} d s
$$

Nominating

$$
E_{i}(t)=\int_{0}^{t} \gamma I_{i}(s) \operatorname{sen}(A(t-s)) e^{-(\omega+d)(t-s)} d s
$$

then,

$$
E_{i}^{\prime}(t)=-(\omega+d) E_{i}(t)+A C_{i}(t)
$$

Therefore, using the ideas from Domoshnitsky (2002), the initial time delay system can be reduced to the corresponding ODE system with this particular function.

In the symmetric case, we already known that since the parameters are symmetric the dynamic of the model will be also symmetric, regarding to equal initial conditions, the variables that represent the sub-populations are equal in the respective class for different serotypes. Then, the primary infections are equal, the secondary infections are the same and so on. Thus, we are going to use the symmetry among serotypes in order to reduce the symmetric system.

First, using the ideas of the Billings (2007), we are going to consider the population reached the equilibrium $N^{*}=\frac{1}{k} \ln \left(\frac{r}{d}\right), r>d$. At the equilibrium, we are going to normalize the ODE using $\bar{S}=\frac{S}{N^{*}}, \overline{I_{i}}=\frac{I_{i}}{N^{*}}, \overline{I_{i j}}=\frac{I_{i j}}{N^{*}}, \overline{C_{i}}=\frac{C_{i}}{N^{*}}, \overline{R_{i}}=\frac{R_{i}}{N^{*}}$ and, $\overline{E_{i}}=\frac{E_{i}}{N^{*}}$.

This way, near the equilibrium at symmetric case, the ODE can be rewritten as 


$$
\begin{aligned}
& \bar{S}^{\prime}(t)=d-d \bar{S}-\beta \bar{S}\left(\overline{I_{1}}+\overline{I_{2}}+\overline{I_{12}}+\overline{I_{21}}\right) \\
& {\overline{I_{1}}}^{\prime}(t)=-(d+\gamma) \overline{I_{1}}+\beta \bar{S}\left(\overline{I_{1}}+\overline{I_{21}}\right) \\
& {\overline{I_{2}}}^{\prime}(t)=-(d+\gamma) \overline{I_{2}}+\beta \bar{S}\left(\overline{I_{2}}+\overline{I_{12}}\right) \\
& {\overline{C_{1}}}^{\prime}(t)=-(d+\omega) \overline{C_{1}}+\gamma \overline{I_{1}}-A \overline{E_{1}} \\
& {\overline{C_{2}}}^{\prime}(t)=-(d+\omega) \overline{C_{2}}+\gamma \overline{I_{2}}-A \overline{E_{2}} \\
& {\overline{E_{1}}}^{\prime}(t)=-(\omega+d) \overline{E_{1}}+A \overline{C_{1}} \\
& {\overline{E_{2}}}^{\prime}(t)=-(\omega+d) \overline{E_{2}}+A \overline{C_{2}} \\
& {\overline{R_{1}}}^{\prime}(t)=-d \overline{R_{1}}-\alpha \phi \overline{R_{1}}\left(\overline{I_{2}}+\overline{I_{12}}\right)+\omega \overline{C_{1}}+A \overline{E_{1}} \\
& {\overline{R_{2}}}^{\prime}(t)=-d \overline{R_{2}}-\alpha \phi \overline{R_{2}}\left(\overline{I_{1}}+\overline{I_{21}}\right)+\omega \overline{C_{2}}+A \overline{E_{2}} \\
& {\overline{I_{12}}}^{\prime}(t)=-(d+\gamma) \overline{I_{12}}+\alpha \phi \overline{R_{1}}\left(\overline{I_{2}}+\overline{I_{12}}\right) \\
& {\overline{I_{21}}}^{\prime}(t)=-(d+\gamma) \overline{I_{21}}+\alpha \phi \overline{R_{2}}\left(\overline{I_{1}}+\overline{I_{21}}\right) .
\end{aligned}
$$

Using the symmetry among the serotypes, we reduce the whole system defining new variables

$$
\begin{aligned}
& s=\bar{S}=\frac{S}{N^{*}} \\
& x=\overline{I_{1}}=\overline{I_{2}} \\
& c=\overline{C_{1}}=\overline{C_{2}} \\
& e=\overline{E_{1}}=\overline{E_{2}} \\
& r=\overline{R_{1}}=\overline{R_{2}} \\
& y=\overline{I_{12}}=\overline{I_{21}} .
\end{aligned}
$$

Thus, the endemic equilibrium of the initial system will be the same equilibrium of the following associated reduced model

$$
\begin{aligned}
& s^{\prime}(t)=d-d s-\beta s 2(x+y) \\
& x^{\prime}(t)=-(d+\gamma) x+\beta s(x+y) \\
& c^{\prime}(t)=-(d+\omega) c+\gamma x-A e \\
& e^{\prime}(t)=-(d+\omega) e+A c \\
& r^{\prime}(t)=-\alpha \phi r(x+y)+\omega c-d r+A e \\
& y^{\prime}(t)=-(d+\gamma) y+\alpha \phi r(x+y) .
\end{aligned}
$$

There is still a complexity of the direct calculation of the endemic equilibrium and the eigenvalues, in terms of the dependency of the parameters. Hence, we are going to use the perturbation theory, in an attempt to deal with this complexity. 
It is important to note that $d$, the mortality rate, is small compared to the other parameters. This time, we introduce another small parameter $\mu$, having now, $\mu$ a parameter slightly bigger than $d$, but still small enough to have the other parameters of the system in order of $\frac{1}{\mu}$. However, $d$ is of $\mathcal{O}(\mu)$.

Afterwards, we set the birth rate $\mu$ and, the mortality rate $d$. We rescale the parameters in relation to $\mu$, letting $\beta=\frac{\beta_{0}}{\mu}, \alpha=\frac{\alpha_{0}}{\mu}, \omega=\frac{\omega_{0}}{\mu}, \gamma=\frac{\gamma_{0}}{\mu}$. With these parameters set, we are close enough to our original system at the steady state and its behaviour.

As soon as we remove the mortality rate, we are able to estimate the endemic equilibrium analytically, in terms of the parameters and stability. Once we set the mortality parameter $d=0$ (because $d$ is of $\mathcal{O}(\mu)$ ) simplifies the model near the equilibrium. Moreover, the mortality term has negligible effect on the steady state because it is a small parameter compared to the other parameters. This way, the resulting local dynamics can be reduced to the following associated system

$$
\begin{aligned}
& s^{\prime}=\mu-\beta s 2(x+y) \\
& x^{\prime}=-\gamma x+\beta s(x+y) \\
& c^{\prime}=\gamma x-\omega c-A e \\
& e^{\prime}=-\omega e+A c \\
& r^{\prime}=\alpha \phi r(x+y)+\omega c+A e \\
& y^{\prime}=-\gamma y+\alpha \phi r(x+y) .
\end{aligned}
$$

The qualitatively analysis of this model is an interesting approach of our original system, but it is only valid for small values of the mortality rate and different values of the birth rate, suggesting an attempt to approach the value of the endemic equilibrium, not including the mortality in the long time dynamic and a possible estimation analysis of the Hopf bifurcation structure for values of $\phi$.

The endemic equilibrium of the system (4.7), considering, $x \neq 0$ and $y \neq 0$, in other words, when the disease is already established, without the mortality term, now it is easy to calculate. And, it is given by

$$
E_{S}=\left(\frac{\gamma_{0}}{2 \beta_{0}}, \frac{\mu^{2}}{2 \gamma_{0}}, \frac{\mu^{2} \omega_{0}}{2\left(\omega_{0}^{2}+A^{2} \mu^{2}\right)}, \frac{\gamma_{0}}{2 \alpha_{0} \phi}, \frac{\mu^{2}}{2 \gamma_{0}}\right)
$$

Now, we use the linearisation theory to analyse the stability of the endemic equilibrium. The Jacobian matrix of the reduced associated system 4.7) at the steady state $E_{S}$ is given by 


$$
J\left(E_{S}\right)=\left[\begin{array}{cccccc}
-\frac{2 \beta_{0} \mu}{\gamma_{0}} & -\frac{\gamma_{0}}{\mu} & 0 & 0 & 0 & -\frac{\gamma_{0}}{\mu} \\
\frac{\beta_{0} \mu}{\gamma_{0}} & -\frac{\gamma_{0}}{2 \mu} & 0 & 0 & 0 & \frac{\gamma_{0}}{2 \mu} \\
0 & \frac{\gamma_{0}}{\mu} & -\frac{\omega_{0}}{\mu} & -A & 0 & 0 \\
0 & 0 & A & -\frac{\omega_{0}}{\mu} & 0 & 0 \\
0 & -\frac{\gamma_{0}}{2 \mu} & \frac{\omega_{0}}{\mu} & A & -\frac{\alpha_{0} \phi \mu}{\gamma_{0}} & -\frac{\gamma_{0}}{2 \mu} \\
0 & \frac{\gamma_{0}}{2 \mu} & 0 & 0 & \frac{\alpha_{0} \phi \mu}{\gamma_{0}} & -\frac{\gamma_{0}}{2 \mu}
\end{array}\right]
$$

with the characteristic polynomial $m(\lambda)=m_{0}+m_{1} \lambda+m_{2} \lambda^{2}+m_{3} \lambda^{3}+m_{4} \lambda^{4}+m_{5} \lambda^{5}+m_{6} \lambda^{6}$ with the coefficients given by

$$
\begin{aligned}
m_{0} & =\frac{2 \alpha_{0} \phi \beta_{0}\left(\omega_{0}^{2}+A^{2} \mu^{2}\right)}{\mu^{2}} \\
m_{1} & =\frac{4 A^{2} \alpha_{0} \phi \beta_{0} \mu}{\gamma_{0}}+\frac{\beta_{0} \gamma \omega_{0}^{2}}{\mu^{3}}+\frac{\beta_{0}\left(A^{2} \gamma_{0}^{2}+4 \alpha_{0} \phi \omega_{0}\left(\gamma_{0}+\omega_{0}\right)\right)}{\gamma_{0} \mu} \\
m_{2} & =\frac{2 A^{2} \alpha_{0} \phi \beta_{0} \mu^{2}}{\gamma_{0}^{2}}+\frac{\gamma_{0}^{2} \omega_{0} \alpha \phi\left(\gamma_{0}+3 \omega_{0}\right)+2 \beta_{0} \omega_{0} \gamma_{0}^{2}\left(2 \gamma_{0}+3 \omega_{0}\right)}{2 \gamma_{0}^{2} \mu^{2}} \\
& +\frac{6 A^{2} \beta_{0} \gamma_{0}^{2}+3 A^{2} \alpha_{0} \phi \gamma_{0}^{2}+4 \alpha_{0} \phi \beta_{0}\left(\gamma_{0}^{2}+4 \gamma_{0} \omega_{0}+\omega_{0}^{2}\right)}{2 \gamma_{0}^{2}} \\
m_{3} & =\frac{\gamma_{0} \omega_{0}^{2}}{\mu^{3}}+\frac{\mu\left(8 \alpha_{0} \phi \beta_{0} \gamma_{0}+2 A^{2} \gamma_{0}\left(2 \beta_{0}+\alpha_{0} \phi\right)+8 \alpha_{0} \phi \beta_{0} \omega_{0}\right)}{2 \gamma_{0}^{2}} \\
& +\frac{2 A^{2} \gamma_{0}^{3}+2 \beta_{0} \gamma_{0}^{3}+12 \beta_{0} \gamma_{0}^{2} \omega_{0}+4 \beta_{0} \gamma_{0} \omega_{0}^{2}+\alpha_{0} \phi \gamma_{0}\left(\gamma_{0}^{2}+6 \gamma_{0} \omega_{0}+2 \omega_{0}^{2}\right)}{2 \gamma_{0}^{2} \mu} \\
m_{4} & =\frac{2 \alpha_{0} \beta_{0}}{\gamma_{0}^{2}}+\frac{\omega_{0}\left(2 \gamma_{0}+\omega_{0}\right)}{\mu^{2}}+\frac{2 A^{2} \gamma_{0}^{2}+6 \beta_{0} \gamma_{0}^{2}+8 \beta_{0} \gamma_{0} \omega_{0}+\alpha_{0} \phi \gamma_{0}\left(3 \gamma_{0}+4 \omega_{0}\right)}{2 \gamma_{0}^{2}} \\
m_{5} & =\frac{2 \gamma_{0}^{3} \mu^{2}+4 \beta_{0} \gamma_{0} \mu^{4}+2 \alpha_{0} \phi \gamma_{0} \mu^{4}+4 \gamma^{2} \omega_{0} \mu^{2}}{2 \gamma_{0}^{2} \mu^{3}} \\
m_{6} & =1 .
\end{aligned}
$$

Since the coefficients of the polynomial are of $\operatorname{order} \mathcal{O}\left(1 / \mu^{3}\right)$ we redefine a polynomial $M(\lambda)=$ $\mu^{3} m(\lambda)$. Thus, we apply the regular perturbation theory, assuming that the solutions of the polynomial $M(\lambda)$ are of the form $\lambda=z_{0}+z_{1} \mu+z_{2} \mu^{2}+\mathcal{O}\left(\mu^{3}\right)$.

Substituting the solutions $\lambda$ in the polynomial $M(\lambda)=\mu^{3} m(\lambda)$ and equalising the terms of the same order, we have

$$
\begin{aligned}
z_{0} & =0 \\
z_{1} & =-2 \frac{\alpha_{0} \phi}{\gamma_{0}} \\
z_{2} & =0
\end{aligned}
$$

and, 


$$
\begin{aligned}
& z^{2}=-\beta_{0} \\
& z_{1}=-\frac{1}{4 \gamma_{0} \omega_{0}}\left[\alpha_{0} \phi\left(\gamma_{0}-\omega_{0}\right)+4 \beta_{0} \omega_{0}\right] \\
& z_{2}= \pm v_{0} \sqrt{\beta_{0}} i
\end{aligned}
$$

where $\left.v_{0}=\frac{1}{32 \beta \gamma^{2} \omega^{2}}\left[16 A^{2} \beta(\gamma-1) \gamma^{2}-16 \beta^{2} \omega^{2}-(\alpha \phi)^{2}\left(\gamma^{2}+14 \gamma \omega-15 \omega^{2}\right)+8 \beta \alpha \phi\left(\gamma^{2}-\omega^{2}\right)\right)\right]$.

Thus, the approximation of the $\mathcal{O}\left(\mu^{3}\right)$ of the eigenvalues are

$$
\lambda_{1}=-2 \frac{\alpha_{0} \phi}{\gamma_{0}} \mu<0
$$

and,

$$
\lambda_{2,3}=-\frac{1}{4 \gamma_{0} \omega_{0}}\left[\alpha_{0} \phi\left(\gamma_{0}-\omega_{0}\right)+4 \beta_{0} \omega_{0}\right] \mu \pm\left(1+v_{0} \mu^{2}\right) \sqrt{\beta_{0}} i
$$

with the negative real part, because $\omega \leq \gamma$, in other words, the length of the recovery time is smaller than the length of the cross immunity time, according to the biological references.

The other eigenvalues can be determined by verifying their magnitude, by analysing the coefficients of the characteristic polynomial. Performing this analysis, it is possible to verify that the other solutions of the polynomial $m(\lambda)$ are of the order $\mathcal{O}\left(\frac{1}{\mu}\right)$. Dividing $m(\lambda)$ by the roots found, it can be noticed that the real root $\lambda_{4}$ is the form of

$$
-\frac{1}{3 \mu}(\gamma+2 \omega)+\frac{\alpha \phi}{3}(\gamma+\omega) \mu+\mathcal{O}(u)
$$

while, the real part of the complex roots has the form

$$
\frac{-1}{3 \mu}(\gamma+2 \omega)+\frac{\alpha \phi}{3}(\gamma+\omega) \mu+\frac{\sqrt[3]{2} \mu}{2 \gamma}+\mathcal{O}\left(u^{2}\right)
$$

Thus, the real part of the eigenvalue is negative since the term of order $\mathcal{O}\left(\frac{1}{\mu}\right)$ is negative.

Thus, it is possible to conclude that the symmetry of the parameters and variables lead to a great reduced system, however it is not possible to find the bifurcation structure in the symmetry. Also, with this conditions, we easily verify numerically that for all values of $\phi>0$ the eigenvalues of this reduced associated model are always negative, showing a stable dynamic near the endemic equilibrium, which does not necessarily occur, as we have seen numerically in the symmetric case for the initial system, over the previous chapter.

Therefore, over this case, we must deal with the whole system without the assumption that the variables are symmetric, only with the assumption the parameters have the symmetry, since the symmetry among the variables does not reflect the stability of the whole system. 
In fact, it is the assumption of the symmetry in the variables and not the perturbation in the mortality term, that makes only the stable dynamic appears for the system. We can assure this statement with numerical results of the stability of the system (4.6), at symmetric case, with symmetry in the variables and without the perturbation of the mortality term. It is possible to see that the eigenvalues of Jacobian matrix at the endemic equilibrium of the system (4.6) have always negative real part, independent of the size of the parameter $\phi$ as demonstrated on the following figures. 


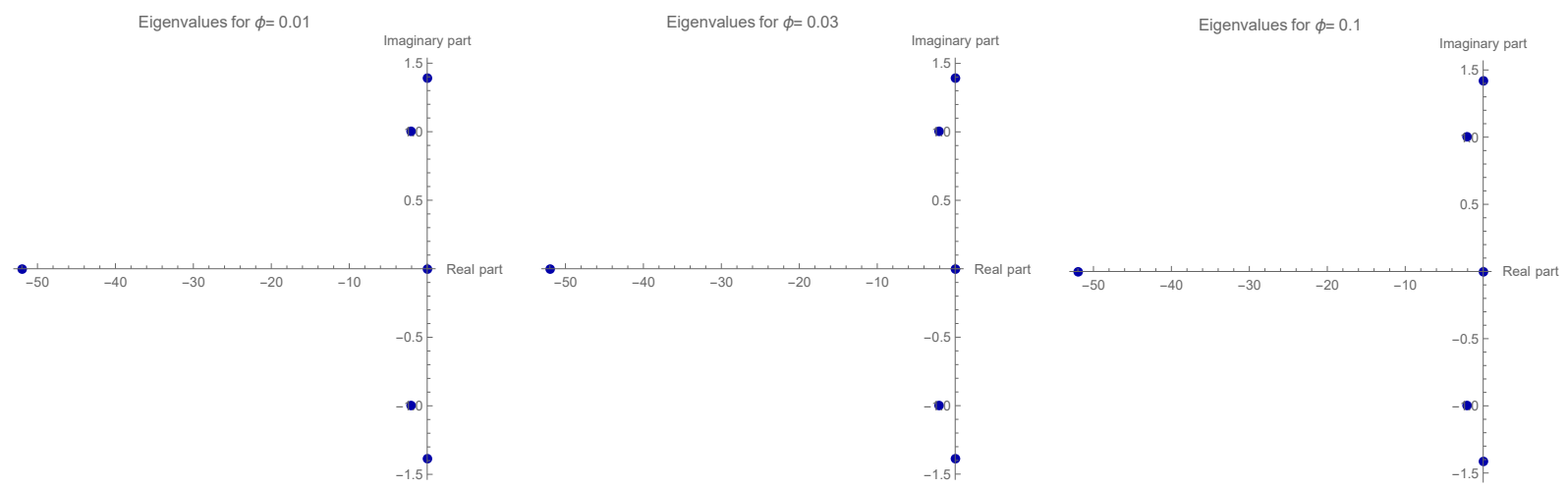
(a) $\phi=0.01$
(b) $\phi=0.03$
(c) $\phi=0.1$

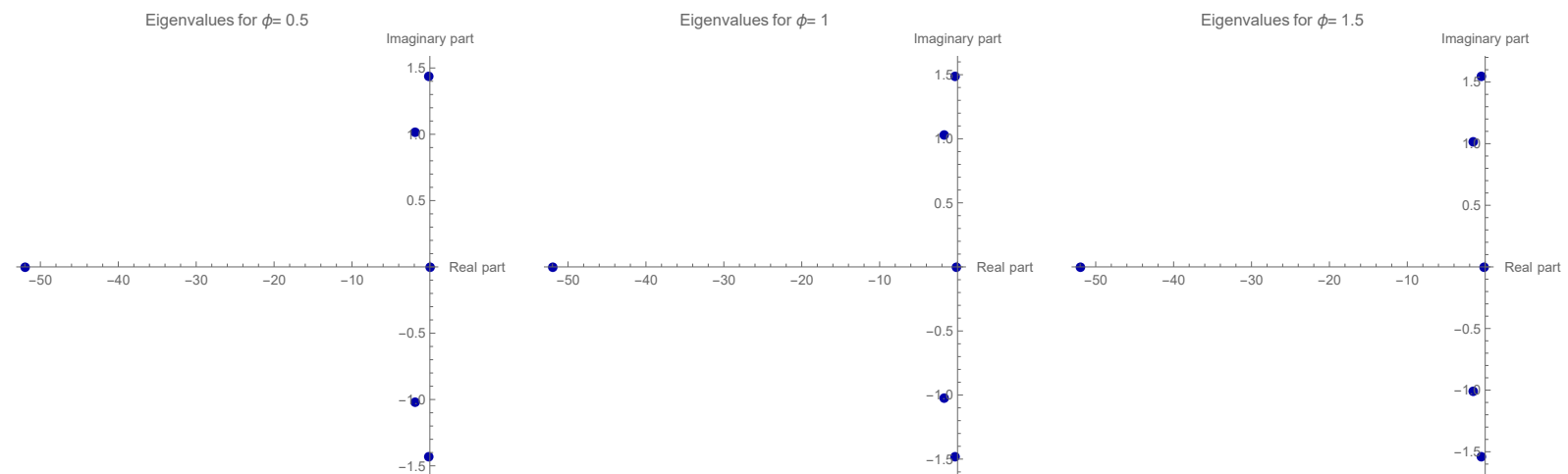
(d) $\phi=0.5$
(e) $\phi=1$
(f) $\phi=1.5$

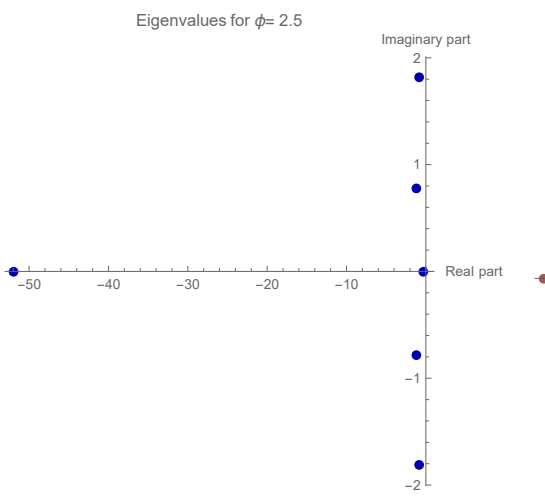

Eigenvalues for $\phi=3.5$

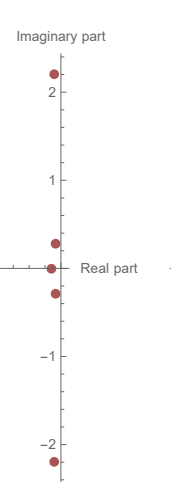

Eigenvalues for $\phi=5$
(g) $\phi=2.5$
(h) $\phi=3.5$
(i) $\phi=5$

Figure 4.1: The figures show the eigenvalues of the endemic equilibrium in the complex plane, for each value of $\phi$, at symmetric case, for symmetric system (4.6) (with symmetry in the variables and without the perturbation in the mortality term). The values used in the simulations are found on table $(3.2)$ with $\beta_{1}=\beta_{2}=180$.

Then, we are going to proceed and work with the whole system (4.4). As a matter of simplicity, we are going to define new variables as following 


$$
\begin{aligned}
s & =\bar{S}=\frac{S}{N^{*}} \\
x_{i} & =\overline{I_{i}} \\
c_{i} & =\overline{C_{i}} \\
e_{i} & =\overline{E_{i}} \\
r_{i} & =\overline{R_{i}} \\
y_{1} & =\overline{I_{21}} \\
y_{2} & =\overline{I_{12}} .
\end{aligned}
$$

Thus, the endemic equilibrium in the initial system will be the same equilibrium of the following associated system

$$
\begin{aligned}
& s^{\prime}(t)=d-d s-\beta s\left(x_{1}+x_{2}+y_{1}+y_{2}\right) \\
& x_{1}^{\prime}(t)=-(d+\gamma) x_{1}+\beta s\left(x_{1}+y_{1}\right) \\
& x_{2}^{\prime}(t)=-(d+\gamma) x_{2}+\beta s\left(x_{2}+y_{2}\right) \\
& c_{1}^{\prime}(t)=-(d+\omega) c_{1}+\gamma x_{1}-A e_{1} \\
& c_{2}^{\prime}(t)=-(d+\omega) c_{2}+\gamma x_{2}-A e_{2} \\
& e_{1}^{\prime}(t)=-(d+\omega) e_{1}+A c_{1} \\
& e_{2}^{\prime}(t)=-(d+\omega) e_{2}+A c_{2} \\
& r_{1}^{\prime}(t)=-\alpha \phi r_{1}\left(x_{2}+y_{2}\right)+\omega c_{1}-d r_{1}+A e_{1} \\
& r_{2}^{\prime}(t)=-\alpha \phi r_{2}\left(x_{1}+y_{1}\right)+\omega c_{2}-d r_{2}+A e_{2} \\
& y_{1}^{\prime}(t)=-(d+\gamma) y_{1}+\alpha \phi r_{2}\left(x_{1}+y_{1}\right) \\
& y_{2}^{\prime}(t)=-(d+\gamma) y_{2}+\alpha \phi r_{1}\left(x_{2}+y_{2}\right) .
\end{aligned}
$$

Now, we are going to use the same idea used before for the reduced model. We are going to use the perturbation theory. Note that $d$, the mortality rate, is small compared to the other parameters. In the sequence, we introduce another small parameter $\mu$, having now, $d$ is of $\mathcal{O}(\mu)$ and, the other parameters of the system in order of $\frac{1}{\mu}$.

Afterwards, we rescale the parameters in relation to $\mu$, letting $\beta=\frac{\beta_{0}}{\mu}, \alpha=\frac{\alpha_{0}}{\mu}, \omega=\frac{\omega_{0}}{\mu}$, $\gamma=\frac{\gamma_{0}}{\mu}$, and setting the birth rate $\mu$ and, the mortality rate $d$. As soon as we have these parameters set, we are close enough to our original system at the steady state and its behaviour.

After removing the mortality rate, we are able to estimate the endemic equilibrium analytically, in terms of the parameters and stability. Once we set the mortality parameter $d=0$ (because $d$ is of $\mathcal{O}(\mu)$ ) simplifies the model near the equilibrium. Moreover, the mortality term has negligible effect on 
the steady state because it is a small parameter compared to the other parameters. Then, the resulting local dynamics can be described by the following system

$$
\begin{aligned}
s^{\prime}(t) & =\mu-\beta s\left(x_{1}+x_{2}+y_{1}+y_{2}\right) \\
x_{1}^{\prime}(t) & =-\gamma x_{1}+\beta s\left(x_{1}+y_{1}\right) \\
x_{2}^{\prime}(t) & =-\gamma x_{2}+\beta s\left(x_{2}+y_{2}\right) \\
c_{1}^{\prime}(t) & =-\omega c_{1}+\gamma x_{1}-A e_{1} \\
c_{2}^{\prime}(t) & =-\omega c_{2}+\gamma x_{2}-A e_{2} \\
e_{1}^{\prime}(t) & =-\omega_{1} e_{1}+A c_{1} \\
e_{2}^{\prime}(t) & =-\omega_{2} e_{2}+A c_{2} \\
r_{1}^{\prime}(t) & =-\alpha \phi r_{1}\left(x_{2}+y_{2}\right)+\omega c_{1}+A e_{1} \\
r_{2}^{\prime}(t) & =-\alpha \phi r_{2}\left(x_{1}+y_{1}\right)+\omega c_{2}+A e_{2} \\
y_{1}^{\prime}(t) & =-\gamma y_{1}+\alpha \phi r_{2}\left(x_{1}+y_{1}\right) \\
y_{2}^{\prime}(t) & =-\gamma y_{2}+\alpha \phi r_{1}\left(x_{2}+y_{2}\right) .
\end{aligned}
$$

The qualitatively analysis of this model is an interesting approach of our original system, but it is only valid for small values of the mortality rate and different values of the birth rate, suggesting an attempt to approach the value of the endemic equilibrium, not including the mortality in the long time dynamic and, a possible estimation analysis of the Hopf bifurcation structure for values of $\phi$.

The endemic equilibrium of the system 4.18 , considering, $x_{i} \neq 0$ and $y_{i} \neq 0$, in other words, when the disease is already established without the mortality term now, it is easy to calculate and it is given by

$$
E_{A}=\left(\frac{\gamma_{0}}{2 \beta_{0}}, \frac{\mu^{2}}{2 \gamma_{0}}, \frac{\mu^{2}}{2 \gamma_{0}}, \frac{\omega_{0} \mu^{2}}{2\left(\omega_{0}^{2}+A^{2} \mu^{2}\right)}, \frac{\omega_{0} \mu^{2}}{2\left(\omega_{0}^{2}+A^{2} \mu^{2}\right)}, \frac{\gamma_{0}}{2 \alpha_{0} \phi}, \frac{\gamma_{0}}{2 \alpha_{0} \phi}, \frac{\mu^{2}}{2 \gamma_{0}}, \frac{\mu^{2}}{2 \gamma_{0}}\right) .
$$

Now, we use the linearisation theory to analyse the stability of the endemic equilibrium. The Jacobian matrix of the reduced associated system (4.18) at the steady state $E_{A}$ is given by 


$$
J\left(E_{A}\right)=\left[\begin{array}{ccccccccccc}
-\frac{2 \beta_{0} \mu}{\gamma_{0}} & -\frac{\gamma_{0}}{2 \mu} & -\frac{\gamma_{0}}{2 \mu} & 0 & 0 & 0 & 0 & 0 & 0 & -\frac{\gamma_{0}}{2 \mu} & -\frac{\gamma_{0}}{2 \mu} \\
\frac{\beta_{0} \mu}{\gamma_{0}} & -\frac{\gamma_{0}}{2 \mu} & 0 & 0 & 0 & 0 & 0 & 0 & 0 & \frac{\gamma_{0}}{2 \mu} & 0 \\
\frac{\beta_{0} \mu}{\gamma_{0}} & 0 & -\frac{\gamma_{0}}{2 \mu} & 0 & 0 & 0 & 0 & 0 & 0 & 0 & \frac{\gamma_{0}}{2 \mu} \\
0 & \frac{\gamma_{0}}{\mu} & 0 & -\frac{\omega_{0}}{\mu} & 0 & -A & 0 & 0 & 0 & 0 & 0 \\
0 & 0 & \frac{\gamma_{0}}{\mu} & 0 & -\frac{\omega_{0}}{\mu} & 0 & -A & 0 & 0 & 0 & 0 \\
0 & 0 & 0 & A & 0 & -\frac{\omega_{0}}{\mu} & 0 & 0 & 0 & 0 & 0 \\
0 & 0 & 0 & 0 & A & 0 & -\frac{\omega_{0}}{\mu} & 0 & 0 & 0 & 0 \\
0 & 0 & -\frac{\gamma_{0}}{2 \mu} & \frac{\omega_{0}}{\mu} & 0 & A & 0 & -\frac{\alpha_{0} \phi \mu}{\gamma_{0}} & 0 & 0 & -\frac{\gamma_{0}}{2 \mu} \\
0 & -\frac{\gamma_{0}}{2 \mu} & 0 & 0 & \frac{\omega_{0}}{\mu} & 0 & A & 0 & -\frac{\alpha_{0} \phi \mu}{\gamma_{0}} & -\frac{\gamma_{0}}{2 \mu} & 0 \\
0 & \frac{\gamma_{0}}{2 \mu} & 0 & 0 & 0 & 0 & 0 & 0 & \frac{\alpha_{0} \phi \mu}{\gamma_{0}} & -\frac{\gamma_{0}}{2 \mu} & 0 \\
0 & 0 & \frac{\gamma_{0}}{2 \mu} & 0 & 0 & 0 & 0 & \frac{\alpha_{0} \phi \mu}{\gamma_{0}} & 0 & 0 & -\frac{\gamma_{0}}{2 \mu}
\end{array}\right] .
$$

It gives a characteristic polynomial $n(\lambda)$ of order 11 , which is very difficult to find all the roots. Our main goal here is to simplify the assumptions as much as possible, in order to get analytical results found in the numerical approach. The reduced model and characteristic polynomial of the reduced model obtained through the symmetry in the variables, among the serotypes, are a particular case of the model (4.18) and its characteristic polynomial. Since in the reduced model there is no bifurcation structure, we will only work with the quotient polynomial $\frac{m(\lambda)}{n(\lambda)}=r(\lambda)$.

If there is a bifurcation structure, thus, it can be only found in the quotient polynomial $r(\lambda)=$ $r_{0}+r_{1} \lambda+r_{2} \lambda^{2}+r_{3} \lambda^{3}+r_{4} \lambda^{4}+r_{5} \lambda^{5}$, where

$$
\begin{aligned}
& r_{0}=-\alpha_{0} \phi \gamma_{0}\left(\frac{\omega_{0}^{2}}{\mu^{3}}+\frac{A^{2}}{\mu}\right) \\
& r_{1}=-\frac{3 \alpha_{0} \phi \omega\left(\gamma_{0}+\omega_{0}\right)}{2 \mu^{2}}-\frac{3 \alpha \phi A^{2}}{2} \\
& r_{2}=-\alpha_{0} \phi A^{2} \frac{\mu}{\gamma_{0}}-\frac{\gamma_{0} \omega_{0}^{2}}{\mu^{3}}-\frac{A^{2} \gamma_{0}+3 \alpha_{0} \phi \omega_{0}}{\mu}-\frac{\alpha_{0} \phi\left(\gamma_{0}^{2}+2 \omega_{0}^{2}\right)}{2 \gamma_{0} \mu} \\
& r_{3}=-A^{2}-\frac{3 \alpha_{0} \phi}{2}-\frac{2 \alpha_{0} \phi \omega_{0}}{\gamma_{0}}-\frac{2 \gamma_{0} \omega_{0}+\omega_{0}^{2}}{\mu^{2}} \\
& r_{4}=-\frac{\alpha_{0} \phi \mu}{\gamma_{0}}-\frac{\gamma_{0}+2 \omega_{0}}{\mu} \\
& r_{5}=-1
\end{aligned}
$$

since, $m(\lambda)$ only shows eigenvalues with negatives real part.

The coefficients of the polynomial are of order $\mathcal{O}\left(1 / \mu^{3}\right)$ we redefine a polynomial $R(\lambda)=$ $\mu^{3} r(\lambda)$. Thus, we apply the regular perturbation theory, assuming that the solutions of the polynomial $R(\lambda)$ are of the form $\lambda=z_{0}+z_{1} \mu+z_{2} \mu^{2}+\mathcal{O}\left(\mu^{3}\right)$. 
Substituting the solutions $\lambda$ in the polynomial $R(\lambda)=\mu^{3} r(\lambda)$ and equalising the terms of the same order we have

$$
\begin{aligned}
z_{0}^{2} & =-\alpha_{0} \phi \\
z_{1} & =\frac{\alpha_{0} \phi}{4 \gamma_{0} \omega_{0}}\left[\gamma_{0}-\omega_{0}\right] \\
z_{2} & = \pm v_{1} \sqrt{\alpha_{0} \phi} i
\end{aligned}
$$

where $v_{1}=\alpha_{0} \phi\left(\frac{1}{4 \omega_{0}^{2}}+\frac{1}{\gamma_{0}^{2}}+\frac{1}{4 \gamma_{0} \omega_{0}}\right)$,

Thus, the approximation of the $\mathcal{O}\left(\mu^{3}\right)$ of the eigenvalues are given by

$$
\lambda_{1,2}=\left(\frac{\alpha_{0} \phi}{4 \gamma_{0} \omega_{0}}\left[\gamma_{0}-\omega_{0}\right]\right) \mu \pm\left(1+v_{1} \mu^{2}\right) \sqrt{\alpha_{0} \phi} i
$$

with the positive real part, because $\omega \leq \gamma$, it means, the length of the recovery time is smaller than the length of the cross immunity time, according to the biological references.

Thus, with the system 4.18 , we show that the endemic equilibrium is always unstable. With this analysis, we can also easily verify numerically that for all values of $\phi>0$ the eigenvalues of the whole system are always negative, except for a pair of complex, which has positive real part, showing a unstable dynamic near the endemic equilibrium.

It was not possible to show analytically the bifurcation structure, however it was possible to prove analytically the instability of the endemic equilibrium. It is what happens for the most values of $\phi$ showed numerically, in the symmetric case.

In this particular case, at symmetric case, for all positive values of $\phi$, there is always a pair of complex eigenvalues that has positive real part, showing that the Coexistence Endemic equilibrium is always unstable, leading to complicated dynamic.

Case (ii): $P(s)=(-a s+1) e^{-\omega s}$

We are going to analyse the case when $P(s)=(-a s+1) e^{-\omega s}$, of course this function satisfies the necessary assumptions, which are, $P(0)=1, P(\infty)=0$ and $\int_{0}^{\infty} e^{-d s} P(s) d s<\infty$. Then,

$$
C_{i}(t)=\int_{0}^{t} \gamma I_{i}(s)(-a(t-s)+1) e^{-(\omega+d)(t-s)} d s,
$$

and,

$$
C_{i}^{\prime}(t)=\gamma I_{i}(t)-(d+\omega) C_{i}(t)-a \int_{0}^{t} \gamma I_{i}(s) e^{-(\omega+d)(t-s)} d s .
$$

Nominating

$$
E_{i}(t)=\int_{0}^{t} \gamma I_{i}(s) e^{-(\omega+d)(t-s)} d s
$$

then,

$$
E_{i}^{\prime}(t)=-(\omega+d) E_{i}(t)+\gamma I_{i}(t)
$$


Therefore, using the ideas from Domoshnitsky (2002), the initial time delay system can be reduced to the corresponding ODE system with this particular function.

At the symmetric case, we already known that since the parameters are symmetric the dynamic of the model will be also symmetric, it means that, for equal initial conditions, the variables which represent the sub-populations are equal in the respective class for different serotypes. Then, the primary infections are equal, the secondary infections are the same and so on. Thus, we are going to use the symmetry among serotypes, in order to reduce the symmetric system.

First, using the ideas from Billings (2007), we consider the population reached the equilibrium $N^{*}=\frac{1}{k} \ln \left(\frac{r}{d}\right), r>d$. At the equilibrium, we normalize the ODE, putting $\bar{S}=\frac{S}{N^{*}}, \overline{I_{i}}=\frac{I_{i}}{N^{*}}, \overline{I_{i j}}=\frac{I_{i j}}{N^{*}}$, $\overline{C_{i}}=\frac{C_{i}}{N^{*}}, \overline{R_{i}}=\frac{R_{i}}{N^{*}}$ and, $\overline{E_{i}}=\frac{E_{i}}{N^{*}}$.

This way, near the equilibrium, at symmetric case, the ODE can be rewritten as

$$
\begin{aligned}
& \bar{S}^{\prime}(t)=d-d \bar{S}-\beta \bar{S}\left(\overline{I_{1}}+\overline{I_{2}}+\overline{I_{12}}+\overline{I_{21}}\right) \\
& {\overline{I_{1}}}^{\prime}(t)=-(d+\gamma) \overline{I_{1}}+\beta \bar{S}\left(\overline{I_{1}}+\overline{I_{21}}\right) \\
& {\overline{I_{2}}}^{\prime}(t)=-\left(d+\gamma \overline{I_{2}}+\beta \bar{S}\left(\overline{I_{2}}+\overline{I_{12}}\right)\right. \\
& {\overline{C_{1}}}^{\prime}(t)=-(d+\omega) \overline{C_{1}}+\gamma \overline{I_{1}}-a \overline{E_{1}} \\
& {\overline{C_{2}}}^{\prime}(t)=-(d+\omega) \overline{C_{2}}+\gamma \overline{I_{2}}-a \overline{E_{2}} \\
& {\overline{E_{1}}}^{\prime}(t)=-(\omega+d) \overline{E_{1}}+\gamma \overline{I_{1}} \\
& {\overline{E_{2}}}^{\prime}(t)=-(\omega+d) \overline{E_{2}}+\gamma \overline{I_{2}} \\
& {\overline{R_{1}}}^{\prime}(t)=-d \overline{R_{1}}-\alpha \phi \overline{R_{1}}\left(\overline{I_{2}}+\overline{I_{12}}\right)+\omega \overline{C_{1}}+a \overline{E_{1}} \\
& {\overline{R_{2}}}^{\prime}(t)=-d \overline{R_{2}}-\alpha \phi \overline{R_{2}}\left(\overline{I_{1}}+\overline{I_{21}}\right)+\omega \overline{C_{2}}+a \overline{E_{2}} \\
& {\overline{I_{12}}}^{\prime}(t)=-(d+\gamma) \overline{I_{12}}+\alpha \phi \overline{R_{1}}\left(\overline{I_{2}}+\overline{I_{12}}\right) \\
& {\overline{I_{21}}}^{\prime}(t)=-(d+\gamma) \overline{I_{21}}+\alpha \phi \overline{R_{2}}\left(\overline{I_{1}}+\overline{I_{21}}\right) .
\end{aligned}
$$

Using the symmetry among the serotypes, we reduce the whole system defining new variables

$$
\begin{aligned}
& s=\bar{S}=\frac{S}{N^{*}} \\
& x=\overline{I_{1}}=\overline{I_{2}} \\
& c=\overline{C_{1}}=\overline{C_{2}} \\
& e=\overline{E_{1}}=\overline{E_{2}} \\
& r=\overline{R_{1}}=\overline{R_{2}} \\
& y=\overline{I_{12}}=\overline{I_{21}} .
\end{aligned}
$$

Thus, the endemic equilibrium of the initial system will be the same equilibrium of the follow- 
ing associated reduced model

$$
\begin{aligned}
& s^{\prime}(t)=d-d s-\beta s 2(x+y) \\
& x^{\prime}(t)=-(d+\gamma) x+\beta s(x+y) \\
& c^{\prime}(t)=-(d+\omega) c+\gamma x-a e \\
& e^{\prime}(t)=-(d+\omega) e+\gamma x \\
& r^{\prime}(t)=-\alpha \phi r(x+y)+\omega c-d r+a e \\
& y^{\prime}(t)=-(d+\gamma) y+\alpha \phi r(x+y) .
\end{aligned}
$$

There is still a complexity of the direct calculation of the endemic equilibrium and the eigenvalues, in terms of the dependency of the parameters. Hence, in an attempt to deal with this complexity we are going to use the perturbation theory.

It is important to note that $d$, the mortality rate, is small compared to the other parameters. This time, we introduce another small parameter $\mu$, having now, $\mu$ a parameter slightly bigger than $d$, but still small enough to have the other parameters of the system in order of $\frac{1}{\mu}$. However, $d$ is of $\mathcal{O}(\mu)$.

We set the birth rate $\mu$ and, the mortality rate $d$ and we rescale the parameters in relation to $\mu$, letting $\beta=\frac{\beta_{0}}{\mu}, \alpha=\frac{\alpha_{0}}{\mu}, \omega=\frac{\omega_{0}}{\mu}, \gamma=\frac{\gamma_{0}}{\mu}$. With this parameters set, we are close enough to our original system at the steady state and its behaviour.

As soon as we remove the mortality rate, we are able to estimate the endemic equilibrium analytically, in terms of the parameters and stability. Once we set the mortality parameter $d=0$ (because $d$ is of $\mathcal{O}(\mu)$ ) simplifies the model near the equilibrium. Moreover, the mortality term has negligible effect on the steady state because it is a small parameter compared to the other parameters. Then, the resulting local dynamics can be reduced to the following associated system

$$
\begin{aligned}
& s^{\prime}=\mu-\beta s 2(x+y) \\
& x^{\prime}=-\gamma x+\beta s(x+y) \\
& c^{\prime}=\gamma x-\omega c-a e \\
& e^{\prime}=-(\omega+d) e+\gamma x \\
& r^{\prime}=\alpha \phi r(x+y)+\omega c+a e \\
& y^{\prime}=-\gamma y+\alpha \phi r(x+y) .
\end{aligned}
$$

The qualitatively analysis of this model is an interesting approach of our original system, but it is only valid for small values of the mortality rate and different values of the birth rate, suggesting an attempt to approach the value of the endemic equilibrium, not including the mortality in the long time dynamic and a possible estimation analysis of the Hopf bifurcation structure for values of $\phi$. 
The endemic equilibrium of the system (4.28) considering $x \neq 0$ and $y \neq 0$, in other words, when the disease is already established, without the mortality term, now it is easy to calculate and it is given by

$$
E_{S}=\left(\frac{\gamma_{0}}{2 \beta_{0}}, \frac{\mu^{2}}{2 \gamma_{0}}, \frac{\mu^{2}}{2 \omega_{0}}\left(\frac{\omega_{0}-a \mu}{\omega_{0}}\right), \frac{\gamma_{0}}{2 \alpha_{0} \phi}, \frac{\mu^{2}}{2 \gamma_{0}}\right)
$$

Now, we use the linearisation theory to analyse the stability of the endemic equilibrium. The Jacobian matrix of the reduced associated system 4.28) at the steady state $E_{S}$ is given by

$$
J\left(E_{S}\right)=\left[\begin{array}{cccccc}
-\frac{2 \beta_{0} \mu}{\gamma_{0}} & -\frac{\gamma_{0}}{\mu} & 0 & 0 & 0 & -\frac{\gamma_{0}}{\mu} \\
\frac{\beta_{0} \mu}{\gamma_{0}} & -\frac{\gamma_{0}}{2 \mu} & 0 & 0 & 0 & \frac{\gamma_{0}}{2 \mu} \\
0 & \frac{\gamma_{0}}{\mu} & -\frac{\omega_{0}}{\mu} & -a & 0 & 0 \\
0 & \frac{\gamma_{0}}{\mu} & 0 & -\frac{\omega_{0}}{\mu} & 0 & 0 \\
0 & -\frac{\gamma_{0}}{2 \mu} & \frac{\omega_{0}}{\mu} & a & -\frac{\alpha_{0} \phi \mu}{\gamma_{0}} & -\frac{\gamma_{0}}{2 \mu} \\
0 & \frac{\gamma_{0}}{2 \mu} & 0 & 0 & \frac{\alpha_{0} \phi \mu}{\gamma_{0}} & -\frac{\gamma_{0}}{2 \mu}
\end{array}\right]
$$

with the characteristic polynomial $m(\lambda)=m_{0}+m_{1} \lambda+m_{2} \lambda^{2}+m_{3} \lambda^{3}+m_{4} \lambda^{4}+m_{5} \lambda^{5}+m_{6} \lambda^{6}$ with the coefficients given by

$$
\begin{aligned}
& m_{0}=\frac{2 \alpha_{0} \phi \beta_{0} \omega_{0}^{2}}{\mu^{2}} \\
& m_{1}=\frac{\left(4 \alpha_{0} \phi \beta_{0} \gamma_{0}^{2} \omega_{0}+4 \alpha_{0} \phi \beta_{0} \gamma_{0} \omega_{0}^{2}\right) \mu^{2}}{\gamma_{0}^{2} \mu^{3}}+\frac{\beta_{0} \gamma_{0} \omega_{0}^{2}}{\mu^{3}} \\
& m_{2}=-\frac{\alpha_{0} \phi a \gamma_{0}}{2 \mu}+\frac{2 \alpha_{0} \phi \beta_{0}\left(\gamma_{0}^{2}+4 \gamma_{0} \omega_{0}+\omega_{0}^{2}\right)}{\gamma_{0}^{2}}+\frac{\alpha_{0} \phi \omega_{0}\left(\gamma_{0}+3 \omega_{0}\right)+2 \beta_{0} \omega_{0}\left(2 \gamma_{0}+3 \omega_{0}\right)}{2 \mu^{2}} \\
& m_{3}=\frac{\gamma_{0} \omega_{0}^{2}}{\mu^{3}}+\frac{4 \alpha_{0} \phi \beta_{0}\left(\gamma_{0}+\omega_{0}\right) \mu}{\gamma_{0}^{2}}+\frac{\beta_{0}\left(\gamma_{0}^{2}+6 \gamma_{0} \omega_{0}+2 \omega_{0}^{2}\right)}{\gamma_{0} \mu}+\frac{\alpha_{0} \phi\left(\gamma_{0}^{2}+6 \gamma_{0} \omega_{0}+2 \omega_{0}^{2}\right)}{2 \gamma_{0} \mu} \\
& m_{4}=\frac{2 \alpha_{0} \phi \beta_{0} \mu^{2}}{\gamma_{0}^{2}}+\frac{\omega_{0}\left(2 \gamma_{0}+\omega_{0}\right)}{\mu^{2}}+\frac{\beta_{0}\left(6 \gamma_{0}+8 \omega_{0}\right)+\alpha_{0} \phi\left(3 \gamma_{0}+4 \omega_{0}\right)}{2 \gamma_{0}} \\
& m_{5}=\frac{\left(\gamma_{0}+2 \omega_{0}\right) \mu^{2}}{\mu^{3}}+\frac{\left(2 \beta_{0}+\alpha_{0} \phi\right) \mu}{\gamma_{0}} \\
& m_{6}=1 .
\end{aligned}
$$

Since the coefficients of the polynomial are of $\operatorname{order} \mathcal{O}\left(1 / \mu^{3}\right)$ we redefine a polynomial $M(\lambda)=$ $\mu^{3} m(\lambda)$. Thus, we apply the regular perturbation theory, assuming that the solutions of the polynomial $M(\lambda)$ are of the form $\lambda=z_{0}+z_{1} \mu+z_{2} \mu^{2}+\mathcal{O}\left(\mu^{3}\right)$.

When we substitute the solutions $\lambda$ in the polynomial $M(\lambda)=\mu^{3} m(\lambda)$ and equalising the 
terms of the same order, we have

$$
\begin{aligned}
& z_{0}=0 \\
& z_{1}=-2 \frac{\alpha_{0} \phi}{\gamma_{0}} \\
& z_{2}=0
\end{aligned}
$$

and,

$$
\begin{aligned}
& z^{2}=-\beta_{0} \\
& z_{1}=-\frac{1}{4 \gamma_{0} \omega_{0}}\left[\alpha_{0} \phi\left(\gamma_{0}-\omega_{0}\right)+4 \beta_{0} \omega_{0}\right] \\
& z_{2}=\frac{\alpha_{0} \phi a}{4 \omega_{0}^{2}} \pm v_{0} \sqrt{\beta_{0}} i,
\end{aligned}
$$

where $v_{0}=\frac{1}{32 \gamma^{2} \omega^{2} \beta}\left[16 \beta^{2} \omega^{2}+(\alpha \phi)^{2}\left(\gamma^{2}+14 \gamma \omega-15 \omega^{2}\right)-8 \alpha \phi \beta\left(\gamma^{2}-8 \gamma \omega-10 \omega^{2}\right)\right]$.

Thus, the approximation of the $\mathcal{O}\left(\mu^{3}\right)$ of the eigenvalues are

$$
\lambda_{1}=-2 \frac{\alpha_{0} \phi}{\gamma_{0}} \mu<0
$$

and,

$$
\lambda_{2,3}=-\frac{1}{4 \gamma_{0} \omega_{0}}\left[\alpha_{0} \phi\left(\gamma_{0}-\omega_{0}\right)+4 \beta_{0} \omega_{0}\right] \mu+\frac{\alpha_{0} \phi a}{4 \omega_{0}^{2}} \mu^{2} \pm\left(1+v_{0} \mu^{2}\right) \sqrt{\beta_{0}} i
$$

with the negative real part, because $\omega \leq \gamma$, in other words, the length of the recovery time is smaller than the length of the cross immunity time, according to the biological references.

The other eigenvalues can be determined by verifying their magnitude, by analysing the coefficients of the characteristic polynomial. Performing this analysis, it is possible to verify that the other solutions of the polynomial $m(\lambda)$ are of the order $\mathcal{O}\left(\frac{1}{\mu}\right)$. Dividing $m(\lambda)$ by the roots found, it can be noticed that the real root $\lambda_{4}$ is of the form

$$
-\frac{\gamma+2 \omega}{3 \mu}+\frac{\alpha \phi}{6 \gamma \omega^{2}}\left(-a \gamma \mu^{2}+\omega(\gamma+\omega) \mu\right)+\mathcal{O}\left(u^{2}\right)
$$

while the real part of the complex roots are the form

$$
-\frac{\gamma+2 \omega}{3 \mu}+\frac{\alpha \phi}{6 \gamma \omega^{2}}\left(-a \gamma \mu^{2}+\omega(\gamma+\omega) \mu\right)-\frac{2 \gamma(\gamma+\omega)^{2}}{3 \sqrt[3]{4} \alpha \phi\left(\gamma^{2}+\omega^{2}\right) \mu}+\mathcal{O}(u)
$$

Thus, the real part of the eigenvalue is negative since the term of order $\mathcal{O}\left(\frac{1}{\mu}\right)$ is negative.

Thus, it is possible to conclude that the symmetry of the parameters and variables lead to a great reduced system but it is not possible to find the bifurcation structure in the symmetry. Also, with 
these conditions, we easily verify numerically that for all values of $\phi>0$ the eigenvalues of this reduced associated model are always negative, showing a stable dynamic near the endemic equilibrium, which does not necessarily occur as we have seen numerically, in the symmetric case for the initial system, during the previous chapter.

Therefore, in this case we have to deal with the whole system, without the assumption that the variables are symmetric, only that the parameters have the symmetry, since the symmetry among the variables does not reflect the stability of the whole system.

In fact, it is the assumption of the symmetry in the variables and not the perturbation in the mortality term, that makes only the stable dynamic appears for the system. We can assure this statement with numerical results of the stability of the system (4.27), at symmetric case, with symmetry in the variables and without the perturbation of the mortality term. It is possible to see that the eigenvalues of Jacobian matrix at the endemic equilibrium of the system (4.27) have always negative real part, independent of the size of the parameter $\phi$ as showed on the following figures.

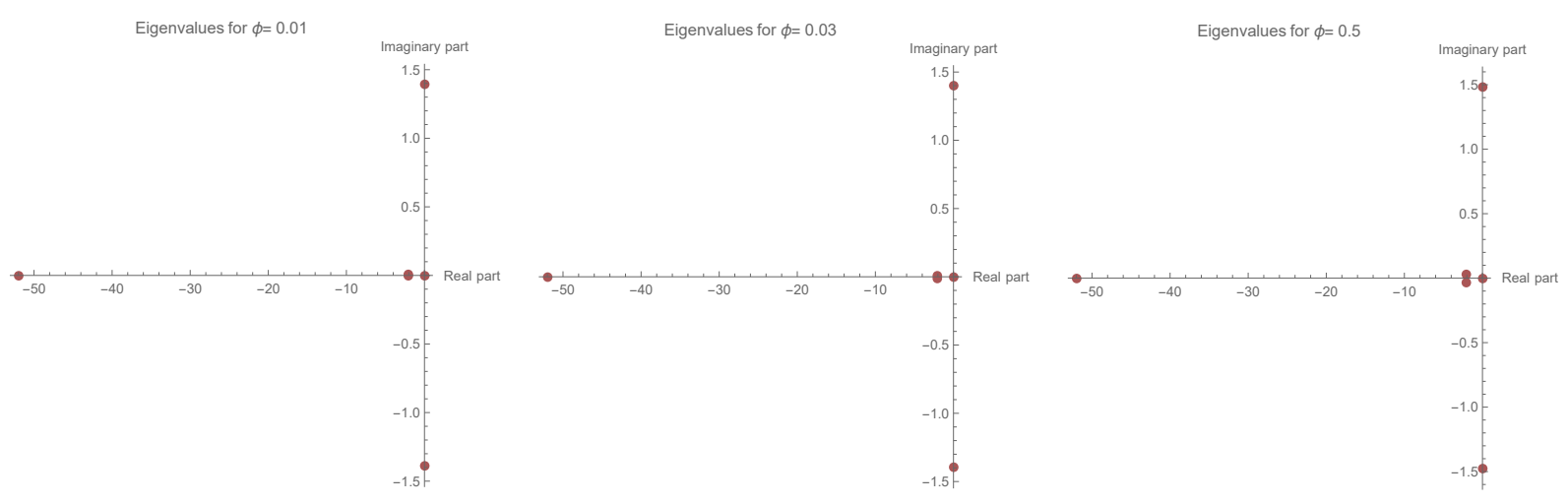
(a) $\phi=0.01$
(b) $\phi=0.03$
(c) $\phi=0.5$

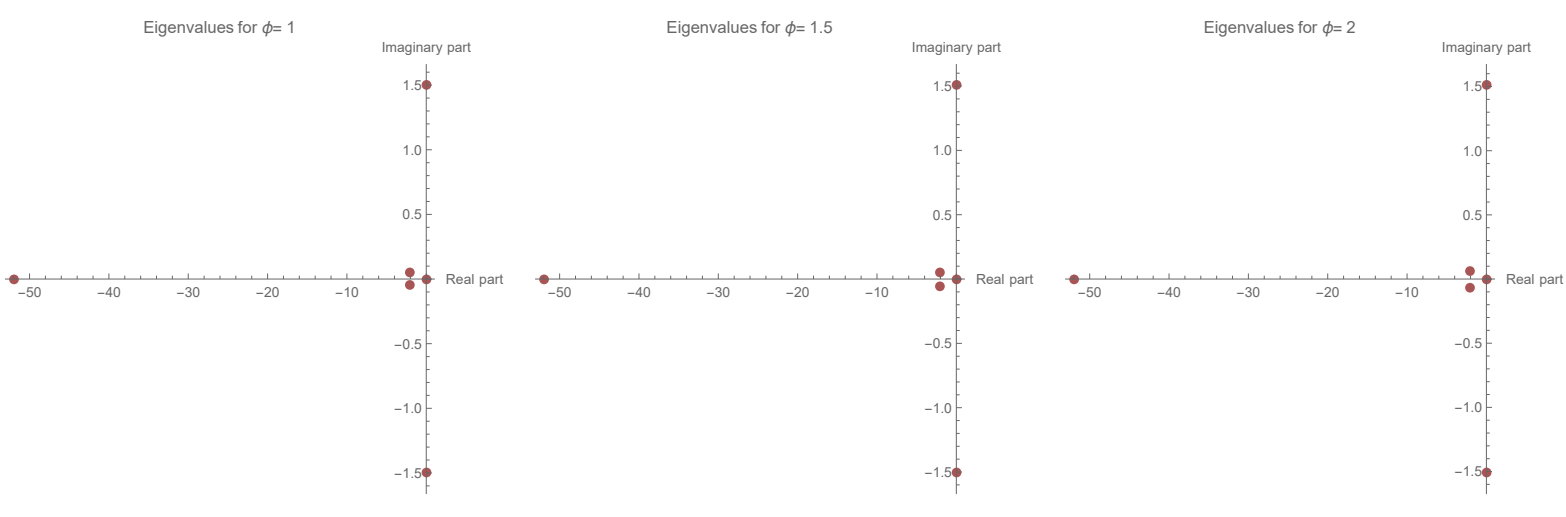
(d) $\phi=1$
(e) $\phi=1.5$
(f) $\phi=2.5$

Figure 4.2: The figures show the eigenvalues of the endemic equilibrium in the complex plane, for each value of $\phi$, at symmetric case, for symmetric system 4.27) ( with symmetry in the variables and without the perturbation in the mortality term). The values used in the simulations are find on table $(3.2)$ with $\beta_{1}=\beta_{2}=180$. 
Then, we are going to proceed and work with the whole system 4.25 . As a matter of simplicity, we are going to define new variables as following

$$
\begin{aligned}
s & =\bar{S}=\frac{S}{N^{*}} \\
x_{i} & =\overline{I_{i}} \\
c_{i} & =\overline{C_{i}} \\
e_{i} & =\overline{E_{i}} \\
r_{i} & =\overline{R_{i}} \\
y_{1} & =\overline{I_{21}} \\
y_{2} & =\overline{I_{12}} .
\end{aligned}
$$

Thus, the endemic equilibrium in the initial system will be the same equilibrium of the following associated system

$$
\begin{aligned}
& s^{\prime}(t)=d-d s-\beta s\left(x_{1}+x_{2}+y_{1}+y_{2}\right) \\
& x_{1}^{\prime}(t)=-(d+\gamma) x_{1}+\beta s\left(x_{1}+y_{1}\right) \\
& x_{2}^{\prime}(t)=-(d+\gamma) x_{2}+\beta s\left(x_{2}+y_{2}\right) \\
& c_{1}^{\prime}(t)=-(d+\omega) c_{1}+\gamma x_{1}-a e_{1} \\
& c_{2}^{\prime}(t)=-(d+\omega) c_{2}+\gamma x_{2}-a e_{2} \\
& e_{1}^{\prime}(t)=-(d+\omega) e_{1}+\gamma x_{1} \\
& e_{2}^{\prime}(t)=-(d+\omega) e_{2}+\gamma x_{2} \\
& r_{1}^{\prime}(t)=-\alpha \phi r_{1}\left(x_{2}+y_{2}\right)+\omega c_{1}-d r_{1}+a e_{1} \\
& r_{2}^{\prime}(t)=-\alpha \phi r_{2}\left(x_{1}+y_{1}\right)+\omega c_{2}-d r_{2}+a e_{2} \\
& y_{1}^{\prime}(t)=-(d+\gamma) y_{1}+\alpha \phi r_{2}\left(x_{1}+y_{1}\right) \\
& y_{2}^{\prime}(t)=-(d+\gamma) y_{2}+\alpha \phi r_{1}\left(x_{2}+y_{2}\right) .
\end{aligned}
$$

Now, we are going to use the same idea used before for the reduced model. We are going to use the perturbation theory. It is important to note that $d$, the mortality rate, is small compared to the other parameters. This way, we introduce another small parameter $\mu$, having now, $d$ is of $\mathcal{O}(\mu)$ and, the other parameters of the system in order of $\frac{1}{\mu}$.

The parameters are rescaled in relation to $\mu$, letting $\beta=\frac{\beta_{0}}{\mu}, \alpha=\frac{\alpha_{0}}{\mu}, \omega=\frac{\omega_{0}}{\mu}, \gamma=\frac{\gamma_{0}}{\mu}$. Afterwards, we set the birth rate $\mu$ and, the mortality rate $d$. With these parameters set, we are close enough to our original system at the steady state and its behaviour. 
As soon as we remove the mortality rate, we are able to estimate the endemic equilibrium analytically, in terms of the parameters and stability. Once we set the mortality parameter $d=0$ (because $d$ is of $\mathcal{O}(\mu)$ ) simplifies the model near the equilibrium. Moreover, the mortality term has negligible effect on the steady state because it is a small parameter compared to the other parameters. Then, the resulting local dynamics can be described by the following system

$$
\begin{aligned}
& s^{\prime}(t)=\mu-\beta s\left(x_{1}+x_{2}+y_{1}+y_{2}\right) \\
& x_{1}^{\prime}(t)=-\gamma x_{1}+\beta s\left(x_{1}+y_{1}\right) \\
& x_{2}^{\prime}(t)=-\gamma x_{2}+\beta s\left(x_{2}+y_{2}\right) \\
& c_{1}^{\prime}(t)=-\omega c_{1}+\gamma x_{1}-a e_{1} \\
& c_{2}^{\prime}(t)=-\omega c_{2}+\gamma x_{2}-a e_{2} \\
& e_{1}^{\prime}(t)=-\omega_{1} e_{1}+\gamma x_{1} \\
& e_{2}^{\prime}(t)=-\omega_{2} e_{2}+\gamma x_{2} \\
& r_{1}^{\prime}(t)=-\alpha \phi r_{1}\left(x_{2}+y_{2}\right)+\omega c_{1}+a e_{1} \\
& r_{2}^{\prime}(t)=-\alpha \phi r_{2}\left(x_{1}+y_{1}\right)+\omega c_{2}+a e_{2} \\
& y_{1}^{\prime}(t)=-\gamma y_{1}+\alpha \phi r_{2}\left(x_{1}+y_{1}\right) \\
& y_{2}^{\prime}(t)=-\gamma y_{2}+\alpha \phi r_{1}\left(x_{2}+y_{2}\right) .
\end{aligned}
$$

The qualitatively analysis of this model is an interesting approach of our original system, but it is only valid for small values of the mortality rate and different values of the birth rate, suggesting an attempt to approach the value of the endemic equilibrium, not including the mortality in the long time dynamic and a possible estimation analysis of the Hopf bifurcation structure for values of $\phi$.

The endemic equilibrium of the system (4.18), considering, $x_{i} \neq 0$ and $y_{i} \neq 0$, it means, when the disease is already established, without the mortality term, now it is easy to calculate. And, it is given by

$$
E_{A}=\left(\frac{\gamma_{0}}{2 \beta_{0}}, \frac{\mu^{2}}{2 \gamma_{0}}, \frac{\mu^{2}}{2 \gamma_{0}}, \frac{\mu^{2}}{2 \omega_{0}^{2}}\left(\omega_{0}-a \mu\right), \frac{\mu^{2}}{2 \omega_{0}^{2}}\left(\omega_{0}-a \mu\right), \frac{\gamma_{0}}{2 \alpha_{0} \phi}, \frac{\gamma_{0}}{2 \alpha_{0} \phi}, \frac{\mu^{2}}{2 \gamma_{0}}, \frac{\mu^{2}}{2 \gamma_{0}}\right) .
$$

Now, we use the linearisation theory to analyse the stability of the endemic equilibrium. The Jacobian matrix of the reduced associated system 4.18 at the steady state $E_{A}$ is given by 


$$
J\left(E_{A}\right)=\left[\begin{array}{ccccccccccc}
-\frac{2 \beta_{0} \mu}{\gamma_{0}} & -\frac{\gamma_{0}}{2 \mu} & -\frac{\gamma_{0}}{2 \mu} & 0 & 0 & 0 & 0 & 0 & 0 & -\frac{\gamma_{0}}{2 \mu} & -\frac{\gamma_{0}}{2 \mu} \\
\frac{\beta_{0} \mu}{\gamma_{0}} & -\frac{\gamma_{0}}{2 \mu} & 0 & 0 & 0 & 0 & 0 & 0 & 0 & \frac{\gamma_{0}}{2 \mu} & 0 \\
\frac{\beta_{0} \mu}{\gamma_{0}} & 0 & -\frac{\gamma_{0}}{2 \mu} & 0 & 0 & 0 & 0 & 0 & 0 & 0 & \frac{\gamma_{0}}{2 \mu} \\
0 & \frac{\gamma_{0}}{\mu} & 0 & -\frac{\omega_{0}}{\mu} & 0 & -a & 0 & 0 & 0 & 0 & 0 \\
0 & 0 & \frac{\gamma_{0}}{\mu} & 0 & -\frac{\omega_{0}}{\mu} & 0 & -a & 0 & 0 & 0 & 0 \\
0 & \frac{\gamma_{0}}{\mu} & 0 & 0 & 0 & -\frac{\omega_{0}}{\mu} & 0 & 0 & 0 & 0 & 0 \\
0 & 0 & \frac{\gamma_{0}}{\mu} & 0 & 0 & 0 & -\frac{\omega_{0}}{\mu} & 0 & 0 & 0 & 0 \\
0 & 0 & -\frac{\gamma_{0}}{2 \mu} & \frac{\omega_{0}}{\mu} & 0 & a & 0 & -\frac{\alpha_{0} \phi \mu}{\gamma_{0}} & 0 & 0 & -\frac{\gamma_{0}}{2 \mu} \\
0 & -\frac{\gamma_{0}}{2 \mu} & 0 & 0 & \frac{\omega_{0}}{\mu} & 0 & a & 0 & -\frac{\alpha_{0} \phi \mu}{\gamma_{0}} & -\frac{\gamma_{0}}{2 \mu} & 0 \\
0 & \frac{\gamma_{0}}{2 \mu} & 0 & 0 & 0 & 0 & 0 & 0 & \frac{\alpha_{0} \phi \mu}{\gamma_{0}} & -\frac{\gamma_{0}}{2 \mu} & 0 \\
0 & 0 & \frac{\gamma_{0}}{2 \mu} & 0 & 0 & 0 & 0 & \frac{\alpha_{0} \phi \mu}{\gamma_{0}} & 0 & 0 & -\frac{\gamma_{0}}{2 \mu}
\end{array}\right] .
$$

It gives a characteristic polynomial $n(\lambda)$ of order 11 , which is very difficult to find all the roots. The main goal here is to simplify the assumptions as much as possible, in order to get analytical results found in the numerical approach. The reduced model and characteristic polynomial of the reduced model obtained through the symmetry in the variables among the serotypes are a particular case of the model (4.18) and its characteristic polynomial. Since in the reduced model there is no bifurcation structure we will only work with the quotient polynomial $\frac{m(\lambda)}{n(\lambda)}=r(\lambda)$.

If there is a bifurcation structure, thus, it can be only found in the quotient polynomial $r(\lambda)=$ $r_{0}+r_{1} \lambda+r_{2} \lambda^{2}+r_{3} \lambda^{3}+r_{4} \lambda^{4}+r_{5} \lambda^{5}$, where

$$
\begin{aligned}
& r_{0}=-\alpha_{0} \phi \gamma_{0} \frac{\omega_{0}^{2}}{\mu^{3}} \\
& r_{1}=-\frac{\alpha_{0} \phi a \gamma_{0}}{2 \mu}-\frac{3 \alpha_{0} \phi \omega_{0}\left(\gamma_{0}+\omega_{0}\right)}{2 \mu^{2}} \\
& r_{2}=-\frac{\alpha_{0} \phi \gamma_{0}}{2 \mu}-\frac{\gamma_{0} \omega_{0}^{2}}{\mu^{3}}-\frac{3 \alpha_{0} \phi \omega_{0}}{\mu}-\frac{\alpha_{0} \phi \omega_{0}^{2}}{\gamma_{0} \mu} \\
& r_{3}=-\frac{3 \alpha_{0} \phi}{2}-\frac{2 \alpha_{0} \phi \omega_{0}}{\gamma_{0}}-\frac{2 \gamma_{0} \omega_{0}}{\mu^{2}}-\frac{\omega_{0}^{2}}{\mu^{2}} \\
& r_{4}=-\frac{\alpha_{0} \phi \mu}{\gamma_{0}}-\frac{\gamma_{0}+2 \omega_{0}}{\mu} \\
& r_{5}=-1
\end{aligned}
$$

since, $m(\lambda)$ only show eigenvalues with negatives real part.

The coefficients of the polynomial are of $\operatorname{order} \mathcal{O}\left(1 / \mu^{3}\right)$ we redefine a polynomial $R(\lambda)=$ $\mu^{3} r(\lambda)$. Thus, we apply the regular perturbation theory, assuming that the solutions of the polynomial $R(\lambda)$ are of the form $\lambda=z_{0}+z_{1} \mu+z_{2} \mu^{2}+\mathcal{O}\left(\mu^{3}\right)$. 
Then, we substitute the solutions $\lambda$ in the polynomial $R(\lambda)=\mu^{3} r(\lambda)$ and equalising the terms of the same order, we have

$$
\begin{aligned}
z_{0}^{2} & =-\alpha_{0} \phi \\
z_{1} & =\frac{\alpha_{0} \phi}{4 \gamma_{0} \omega_{0}}\left[\gamma_{0}-\omega_{0}\right] \\
z_{2} & =-\frac{\alpha_{0} \phi a}{4 \omega_{0}^{2}} \pm v_{1} \sqrt{\alpha_{0} \phi} i .
\end{aligned}
$$

where $v_{1}=\frac{\alpha_{0} \phi}{16}\left(\frac{3}{\omega_{0}^{2}}+\frac{3}{\gamma_{0}^{2}}+\frac{6}{\gamma_{0} \omega_{0}}\right)$,

Thus, the approximation of the $\mathcal{O}\left(\mu^{3}\right)$ of the eigenvalues are given by

$$
\lambda_{1,2}=\left(\frac{\alpha_{0} \phi}{4 \gamma_{0} \omega_{0}}\left[\gamma_{0}-\omega_{0}\right]\right) \mu-\frac{\alpha_{0} \phi a}{4 \omega_{0}^{2}} \mu^{2} \pm\left(1+v_{1} \mu^{2}\right) \sqrt{\alpha_{0} \phi} i
$$

with the positive real part, because $\omega \leq \gamma$, in other words, the length of the recovery time is smaller than the length of the cross immunity time, according to the biological references.

Thus, with the system (4.18), we show that the endemic equilibrium is always unstable. With this analysis, we can also easily verify numerically that for all values of $\phi>0$ the eigenvalues of the whole system are always negative, except for a pair of complex which has positive real part, showing a unstable dynamic near the endemic equilibrium.

It was not possible to show analytically the bifurcation structure, however it was possible to prove analytically the instability of the endemic equilibrium, it is what happens for the most values of $\phi$ showed numerically in the symmetric case.

In this particular case, at symmetric case, for all positive values of $\phi$, there is always a pair of complex eigenvalues that has positive real part, showing that the Coexistence Endemic equilibrium is always unstable, leading to complicated dynamic. 
CHAPTER 5

\section{Perturbations of Non-linear System}

By adding small mortality in the original system, we could better represent the dynamic of Dengue fever with the inherent characteristics. In order to represent small, but possible death caused by disease, we have perturbed the proposed model by adding a mortality term in the infectious classes.

Through perturbation theory of non-linear systems, we are going to analyse this new system of IDE and discuss the results comparing solutions of the proposed system with the new model with mortality term. Furthermore, numerical analysis will support the theoretical study, showing numerical solutions of the perturbed system, for long term behaviour.

\subsection{Adding mortality caused by disease}

In the sequence, we wish to study the following system: 


$$
\begin{aligned}
& \frac{d S(t)}{d t}=r N(t) e^{-k N(t)}-d S(t)-\beta_{1} \frac{S(t)}{N(t)}\left(I_{1}(t)+I_{21}(t)\right)-\beta_{2} \frac{S(t)}{N(t)}\left(I_{2}(t)+I_{12}(t)\right) \\
& \frac{d I_{1}(t)}{d t}=-(d+\gamma) I_{1}(t)+\beta_{1} \frac{S(t)}{N(t)}\left(I_{1}(t)+I_{21}(t)\right)-\mu_{1} I_{1}(t) \\
& \frac{d I_{2}(t)}{d t}=-(d+\gamma) I_{2}(t)+\beta_{2} \frac{S(t)}{N(t)}\left(I_{2}(t)+I_{12}(t)\right)-\mu_{2} I_{2}(t) \\
& \frac{d C_{1}(t)}{d t}=\gamma I_{1}(t)-d C_{1}(t)+\int_{0}^{t} \gamma I_{1}(s) P_{t}^{1}(t-s) e^{-d(t-s)} d s \\
& \frac{d C_{2}(t)}{d t}=\gamma I_{2}(t)-d C_{2}(t)+\int_{0}^{t} \gamma I_{2}(s) P_{t}^{2}(t-s) e^{-d(t-s)} d s \\
& \frac{d R_{1}(t)}{d t}=-d R_{1}(t)-\alpha_{2} \phi \frac{R_{1}(t)}{N(t)}\left(I_{12}(t)+I_{2}(t)\right)-\int_{0}^{t} \gamma I_{1}(s) P_{t}^{1}(t-s) e^{-d(t-s)} d s \\
& \frac{d R_{2}(t)}{d t}=-d R_{2}(t)-\alpha_{1} \phi \frac{R_{2}(t)}{N(t)}\left(I_{21}(t)+I_{1}(t)\right)-\int_{0}^{t} \gamma I_{2}(s) P_{t}^{2}(t-s) e^{-d(t-s)} d s \\
& \frac{d I_{12}(t)}{d t}=-(d+\gamma) I_{12}(t)+\alpha_{2} \phi \frac{R_{1}(t)}{N(t)}\left(I_{2}(t)+I_{12}(t)\right)-\mu_{2} I_{12}(t) \\
& \frac{d I_{21}(t)}{d t}=-(d+\gamma) I_{21}(t)+\alpha_{1} \phi \frac{R_{2}(t)}{N(t)}\left(I_{1}(t)+I_{21}(t)\right)-\mu_{1} I_{21}(t) \\
& \frac{d R(t)}{d t}=-d R(t)+\gamma\left(I_{12}(t)+I_{21}(t)\right)+\mu_{1} I_{1}(t)+\mu_{2} I_{2}(t)+\mu_{1} I_{21}(t)+\mu_{2} I_{12}(t) .
\end{aligned}
$$

The constants $\mu_{i}$, for $i=1,2$, represent a possible but small death caused by the disease and, it will be different for each serotype. We are assuming also, that who dies of the disease is counted as recovered, because it will no longer infect or be susceptible. Note that this new system could be more specific in order to represent the disease and the deaths caused by disease. Mathematically it represents a perturbation of the delay system that we have been studying so far, because when $\mu_{i}=0$ we have the system (1.7).

The total population dynamics is determined by

$$
\frac{d N(t)}{d t}=r N(t) e^{-k N(t)}-d N(t)
$$

where, $N(t)=S(t)+I_{1}(t)+I_{2}(t)+C_{1}(t)+C_{2}(t)+I_{12}(t)+I_{21}(t)+R_{1}(t)+R_{2}(t)+R(t)$.

We let $\frac{S}{N}=s, \frac{I_{i j}}{N}=x_{i j}, \frac{C_{i}}{N}=c_{i}$ and $\frac{R_{i}}{N}=r_{i}$ denoting, for each class, the fractions of the population. Thus, the sum of the total population satisfies $s+x_{1}+x_{2}+c_{1}+c_{2}+r_{1}+r_{2}+x_{12}+x_{21}+r=1$. This way, we assume that the population reached the equilibrium $N^{*}$, the original system can be studied analysing the following subsystem: 


$$
\begin{aligned}
\frac{d s(t)}{d t} & =d-d s(t)-\beta_{1} s(t)\left(x_{1}(t)+x_{21}(t)\right)-\beta_{2} s(t)\left(x_{2}(t)+x_{12}(t)\right) \\
\frac{d x_{1}(t)}{d t} & =-(d+\gamma) x_{1}(t)+\beta_{1} s(t)\left(x_{1}(t)+x_{21}(t)\right)-\mu_{1} x_{1}(t) \\
\frac{d x_{2}(t)}{d t} & =-(d+\gamma) x_{2}(t)+\beta_{2} s(t)\left(x_{2}(t)+x_{12}(t)\right)-\mu_{2} x_{2}(t) \\
\frac{d r_{1}(t)}{d t} & =-d r_{1}(t)-\alpha_{2} \phi r_{1}(t)\left(x_{12}(t)+x_{2}(t)\right)-\int_{0}^{t} \gamma x_{1}(s) P_{t}^{1}(t-s) e^{-d(t-s)} d s \\
\frac{d r_{2}(t)}{d t} & =-d r_{2}(t)-\alpha_{1} \phi r_{2}(t)\left(x_{21}(t)+x_{1}(t)\right)-\int_{0}^{t} \gamma x_{2}(s) P_{t}^{2}(t-s) e^{-d(t-s)} d s \\
\frac{d x_{12}(t)}{d t} & =-(d+\gamma) x_{12}(t)+\alpha_{2} \phi r_{1}(t)\left(x_{2}(t)+x_{12}(t)\right)-\mu_{2} x_{12}(t) \\
\frac{d x_{21}(t)}{d t} & =-(d+\gamma) x_{21}(t)+\alpha_{1} \phi r_{2}(t)\left(x_{1}(t)+x_{21}(t)\right)-\mu_{1} x_{21}(t) .
\end{aligned}
$$

Note that the dynamics for the recovered and cross immunity classes are decoupled, it means we could remove the equations which represent that dynamic and reduce the original system to these seven equations subsystem. Also, working with the fractions of the populations as variables of the system, we do not need to worry with the dynamic of the total population.

For this analyses, we consider $\mu_{i}=\mu$. The system 5.2 is denoted by

$$
y^{\prime}(t)=F(t, y(t))-\int_{0}^{t} \gamma e^{-d(t-s)} P_{t}(t-s) E_{1} y(s) d s-\mu E_{2} y(s)
$$

where $\mu$ represents a constant parameter, $E_{1}$ represents a matrix with all null entries except in the entries correspondents to the functions $P_{t}^{i}(t)$, and $E_{2}$ is also an elementary matrix.

To study this system we are going to apply the theory of perturbation of nonlinear system and, we are going to use the results by Brauer (1966) and Brauer (1972), in order to study the behaviour of the solutions of this new system.

It is important to note that this new system has the same equilibriums that the original system but now, the values of the equilibrium depend on the mortality rate $\mu$. Also, it is important to note that the Basic Reproduction number decrease with the mortality rate, this is, the infection time is now $\frac{1}{d+\gamma+\mu}$.

The next result is an adaptation of results of the Brauer (1966, 1972) and it can give us an insight in order to prove if the mortality rate for the disease is small, then we have continuity in the parameter and the solutions of the perturbed system are close to the original system.

We consider the solution of system (5.2) (or (5.3) as function of $t$, initials conditions, as well as, of parameter $\mu$ and, we will denote $y(t)=y\left(t, t_{0}, y_{0}, \mu\right)$ to indicate this dependence. We can use the ideas of the Brauer (1972) and differentiate the equation (5.3) with respect to the parameter $\mu$. Then,

$$
\frac{\partial}{\partial t} \frac{\partial y(t)}{\partial \mu}=\frac{\partial y^{\prime}(t)}{\partial \mu}=F_{y}(y(t)) \frac{\partial y(t)}{\partial \mu}-\int_{0}^{t} \gamma e^{-d(t-s)} P_{t}(t-s) E_{1} \frac{\partial y(s)}{\partial \mu} d s-\mu E_{2} \frac{\partial y(t)}{\partial \mu}-E_{2} y(t)
$$

It gives the following result. 
Lemma 2. The vector matrix $\frac{\partial y(t)}{\partial \mu}$ whose entries are the partial derivatives of the components of $y(t)$ with respect to the parameter $\mu$ satisfies the integro-differential equation

$$
\begin{aligned}
V^{\prime}\left(t, t_{0}, y_{0}, \mu\right) & =F_{y}(y(t)) V\left(t, t_{0}, y_{0}, \mu\right)-\int_{t_{0}}^{t} \gamma e^{-d(t-s)} P_{t}(t-s) E_{1} V\left(s, t_{0}, y_{0}, \mu\right) d s-\mu E_{2} V\left(t, t_{0}, y_{0}, \mu\right)-E_{2} y(t) \\
V\left(t_{0}\right) & =0
\end{aligned}
$$

The solution $V(t)$ of system (5.5) gives us a limitation estimated for the difference between the solution of perturbed system $[5.3$ and the original system with distributed time delay.

Lemma 3. Let $V\left(t, t_{0}, y_{0}, \mu\right)$ be the solution of system (5.5). If $\mu$ and $\eta$ are in a convex subset $D$, and $V(t)$ is uniform bounded in $\mu$ then, for all $t \geq t_{0}$,

$$
\left|y\left(t, t_{0}, y_{0}, \mu\right)-y\left(t, t_{0}, y_{0}, \eta\right)\right| \leq|V(t)||\mu-\eta|
$$

Proof. Let $\xi$ be the line from $\mu$ to $\eta$, given by $\xi(\lambda)=\mu+\lambda(\eta-\mu)$, for $0 \leq \lambda \leq 1$. Since $D$ is convex, the graph of $\xi$ lies in $D$. Then

$$
\frac{d}{d \lambda}\left[y\left(t, t_{0}, y_{0}, \xi(\lambda)\right)\right]=\frac{\partial}{\partial \xi(\lambda)} y\left(t, t_{0}, y_{0}, \xi(\lambda)\right) \xi^{\prime}(\lambda)=V\left(t, t_{0}, y_{0}, \xi(\lambda)\right)(\eta-\mu) .
$$

Integrating we have

$$
y\left(t, t_{0}, y_{0}, \mu\right)-y\left(t, t_{0}, y_{0}, \eta\right)=\int_{0}^{1} V\left(t, t_{0}, y_{0}, \xi(\lambda)\right) d \lambda(\eta-\mu) .
$$

Since $V(t)$ is uniform bounded in $\mu$, thus we have

$$
\begin{aligned}
\left|y\left(t, t_{0}, y_{0}, \mu\right)-y\left(t, t_{0}, y_{0}, \eta\right)\right| & \leq\left|\int_{0}^{1} V\left(t, t_{0}, y_{0}, \xi(\lambda)\right) d \lambda\right||\eta-\mu| \\
& \leq \max _{0 \leq \lambda \leq 1}\left|V\left(t, t_{0}, y_{0}, \xi(\lambda)\right)\right||\mu-\eta| \\
& \leq|V(t)||\mu-\eta| .
\end{aligned}
$$

The following Theorem relates and gives an estimation for distance between the solutions of the system (5.1) and (1.7).

Theorem 9. The solutions of system (5.3) and (4.4), for sufficiently small $\mu$, are related by

$$
\left|y\left(t, t_{0}, y_{0}\right)-x\left(t, t_{0}, y_{0}\right)\right| \leq \epsilon
$$

for all $t \in[0, T]$.

Proof. First note that $y\left(t, t_{0}, y_{0}, 0\right)=x\left(t, t_{0}, y_{0}\right)$, in other words, the solution with $\eta=0$ is the solution of original system. 
We are going to estimate the limitation of solution $V$. The solution $V\left(t, t_{0}, y_{0}, \mu\right)$ satisfies the system (5.5) where,

$$
F_{y}(y(t))=\left[\begin{array}{ccccccc}
w & -\beta_{1} s & -\beta_{2} s & 0 & 0 & -\beta_{2} s & -\beta_{1} s \\
\beta_{1}\left(x_{1}+x_{21}\right) & w_{3} & 0 & 0 & 0 & \beta_{1} s & 0 \\
\beta_{2}\left(x_{2}+x_{12}\right) & 0 & w_{4} & 0 & 0 & 0 & \beta_{2} s \\
0 & 0 & -\alpha_{2} \phi r_{1} & -\alpha_{2} \phi\left(x_{2}+x_{12}\right)-d & 0 & 0 & -\alpha_{2} \phi r_{1} \\
0 & -\alpha_{1} \phi r_{2} & 0 & 0 & -\alpha_{1} \phi\left(x_{1}+x_{21}\right)-d & -\alpha_{1} \phi r_{2} & 0 \\
0 & \alpha_{1} \phi r_{2} & 0 & 0 & \alpha_{1} \phi\left(x_{1}+x_{21}\right) & w_{1} & 0 \\
0 & 0 & \alpha_{2} \phi r_{1} & \alpha_{2} \phi\left(x_{2}+x_{12}\right) & 0 & 0 & w_{2}
\end{array}\right],(5.9)
$$

and $w=-d-\beta_{1}\left(x_{1}+x_{21}\right)-\beta_{2}\left(x_{2}+x_{12}\right), w_{1}=\alpha_{1} \phi r_{2}-(d+\gamma), w_{2}=\alpha_{2} \phi r_{1}-(d+\gamma)$, $w_{3}=-(d+\gamma)+\beta_{1} s$ and $w_{4}=-(d+\gamma)+\beta_{2} s$. Thus, $\left|F_{y}(y(s))\right| \leq d+\gamma+K_{\beta, \alpha \phi}$, where $K_{\beta, \alpha \phi}=\max \left\{\beta_{1}, \beta_{2}, \alpha_{1} \phi, \alpha_{2} \phi\right\}$.

If $V(t)=V\left(t, t_{0}, y_{0}, \mu\right)$, then

$$
\begin{aligned}
e^{t \mu E_{2}} V^{\prime}(t)+e^{t \mu E_{2}} \mu E_{2} V(t) & = \\
& e^{t \mu E_{2}}\left[F_{y}(y(t)) V(t)-\int_{t_{0}}^{t} \gamma e^{-d(t-s)} P_{t}(t-s) E_{1} V(s) d s-E_{2} y(t)\right] .
\end{aligned}
$$

This implies that

$$
\left(e^{t \mu E_{2}} V(t)\right)^{\prime}=e^{t \mu E_{2}}\left[F_{y}(y(t)) V(t)-\int_{t_{0}}^{t} \gamma e^{-d(t-s)} P_{t}(t-s) E_{1} V(s) d s-E_{2} y(t)\right] .
$$

Integrating from $t_{0}=0$ to $t$ we have

$$
e^{t \mu E_{2}} V(t)=\int_{0}^{t} e^{\tau \mu E_{2}}\left[F_{y}(y(\tau)) V(\tau)-\int_{0}^{\tau} \gamma e^{-d(\tau-s)} P_{\tau}(\tau-s) E_{1} V(s) d s-E_{2} y(\tau)\right] d \tau .
$$

So,

$$
\begin{aligned}
|V(t)| & \leq\left|e^{-t \mu E_{2} \mid}\right| \int_{0}^{t} e^{\tau \mu E_{2}}\left[F_{y}(y(\tau)) V(\tau)-\int_{0}^{\tau} \gamma e^{-d(\tau-s)} P_{\tau}(\tau-s) E_{1} V(s) d s-E_{2} y(\tau)\right] d \tau \mid \\
& \leq e^{-t \mu}\left[\int_{0}^{t}\left|e^{\tau \mu E_{2}}\right|\left[\left|F_{y}(y(\tau))\right||V(\tau)|+\int_{0}^{\tau} \gamma\left|e^{-d(\tau-s)} P_{\tau}(\tau-s) E_{1}\right||V(s)| d s+\left|E_{2} y(\tau)\right|\right] d \tau\right] \\
& \leq e^{-t \mu}\left[\int_{0}^{t} e^{\tau \mu}\left[\left|F_{y}(y(\tau))\right||V(\tau)|+\int_{0}^{\tau} \gamma\left|e^{-d(\tau-s)} P_{\tau}(\tau-s)\right||V(s)| d s+|y(\tau)|\right] d \tau\right] .
\end{aligned}
$$

Therefore,

$$
\begin{aligned}
|V(t)| e^{\mu t} & \leq \int_{0}^{t} e^{\mu \tau}\left|F_{y}(y(\tau))\right||V(\tau)| d \tau+\int_{0}^{t} e^{\mu \tau}\left[\int_{0}^{\tau} \gamma\left(-e^{-d(\tau-s)} P_{\tau}(\tau-s)\right)|V(s)| d s\right] d \tau \\
& +\int_{0}^{t} e^{\mu \tau}|y(\tau)| d \tau .
\end{aligned}
$$

We let $\Delta_{i}=\mu$. Since, $\mu<d$, we have that $\sup _{\tau<0}\left|e^{\mu \tau} y(\tau)\right|$ exists, and in fact $\sup _{\tau<0}\left|e^{\mu \tau} y(\tau)\right|=$ $\left\|y_{t}\right\|_{e}$, defined previously for solution in the Banach space, where the initial conditions are functions defined in $-\infty$. 
Thus,

$$
\begin{aligned}
Z(t) & =|V(t)| e^{\mu t} \\
& \leq \int_{0}^{t}\left|F_{y}(y(\tau))\right| Z(\tau) d \tau+\int_{0}^{t} e^{\mu \tau}\left[\int_{0}^{\tau} \gamma\left(-e^{-d(\tau-s)} P_{\tau}(\tau-s)\right)|V(s)| d s\right] d \tau+\int_{0}^{t}|| y_{t} \mid e_{e} d \tau \\
& \leq\left\|y_{t}\right\|_{e} t+\int_{0}^{t}\left|F_{y}(y(\tau))\right| Z(\tau) d \tau+\int_{0}^{t} e^{\mu \tau}\left[\int_{0}^{\tau} \gamma\left(-e^{-d(\tau-s)} P_{\tau}(\tau-s)\right) e^{\mu(\tau-s)} e^{-\mu(\tau-s)}|V(s)| d s\right] d \tau \\
& \leq\left\|y_{t}\right\|_{e} t+\int_{0}^{t}\left|F_{y}(y(s))\right| Z(s) d s+\int_{0}^{t} e^{\mu \tau}\left[\int_{0}^{\tau} \gamma\left(-e^{(-d+\mu)(\tau-s)} P_{\tau}(\tau-s)\right) e^{-\mu(\tau)} Z(s) d s\right] d \tau \\
& \leq t+\int_{0}^{t}\left|F_{y}(y(s))\right| Z(s) d s+\int_{0}^{t} \int_{s}^{t} e^{\mu \tau} \gamma\left(-e^{(-d+\mu)(\tau-s)} P_{\tau}(\tau-s)\right) e^{-\mu(\tau)} Z(s) d \tau d s \\
& \leq t+\int_{0}^{t}\left[\left|F_{y}(y(s))\right|+\int_{s}^{t} \gamma\left(-e^{(-d+\mu)(\tau-s)} P_{\tau}(\tau-s)\right) d \tau\right] Z(s) d s \\
& \leq t+\int_{0}^{t}\left[\left|F_{y}(y(s))\right|+\int_{0}^{t-s} \gamma e^{(-d+\mu) \xi}\left(-P_{\xi}(\xi)\right) d \xi\right] Z(s) d s .
\end{aligned}
$$

The hypothesis 2.5 guarantees that

$$
\int_{0}^{\infty} \gamma e^{(-d+\mu) \xi}\left(-P_{\xi}(\xi)\right) d \xi<\infty
$$

Thus,

$$
Z(t) \leq t+\int_{0}^{t}\left[d+\gamma+K_{\beta, \alpha \phi}+\gamma M\right] Z(s) d s .
$$

Using Gronwall inequality for $Z(t)$ we have that

$$
|V(t)| \leq t e^{-\mu t} e^{\left[d+\gamma+K_{\beta, \alpha \phi}+\gamma M\right] t} .
$$

Therefore,

$$
|V(t)| \leq e^{\left(2 \gamma+K_{\beta, \alpha \phi}\right) t}=e^{k t} .
$$

is uniform bounded in $\mu$. From Lemma (3) we conclude that

$$
\left|y\left(t, t_{0}, y_{0}, \mu\right)-x\left(t, t_{0}, y_{0}\right)\right| \leq \mu e^{k t} .
$$

Therefore, for each $\epsilon$ and $T>0$ exist $\delta>0$ such that $\mu<\delta$ implies that

$$
\left|y\left(t, t_{0}, y_{0}, \mu\right)-x\left(t, t_{0}, y_{0}\right)\right| \leq \epsilon,
$$

for all $t \in[0, T]$. 
From this theorem, we conclude that to study the behaviour of the system (5.1) is sufficient to know the behaviour of the system (1.7), because of the continuity in the parameter. Thus, to add a small mortality in the system, corresponding to the mortality due to disease in different stages of Dengue, from the point of view of the qualitative study, it does not imply changes in the behaviour of the solutions because both solutions are close for $t \in[0, T]$.

Biologically speaking, this means that small mortality for the disease doesn't affect the qualitatively behaviour of the model as well as the behaviour of the propagation of the disease. Therefore, the original system can be a good representation for the study of the propagation of the disease, even without the mortality term.

\subsection{Numerical Solutions of Long-term Behaviour}

In this chapter we are going to analyse numerically the solutions of the system in order to obtain more information about the dynamic of the perturbed system (5.1), as well as supporting the theoretical study of the previously section.

Here, $\mu_{1}$ will represent the mortality rate caused by disease for infection and reinfection of one sertoype, and $\mu_{2}$ will represent a different value for mortality rate for the infection and reinfection of the other serotype.

The numerical values for the parameters are shown on the table (3.2) at Chapter 3. Additionally, as we are assuming $\beta_{2}>\beta_{1}$, the infecction 2 is more severe than the infection 1 . Thus, we are assuming that the mortality rate for infection 2 is bigger than the other. Therefore, for this simulations were used $\mu_{1}=0.009$ and $\mu_{2}=0.01$. These values were based on information found in WHO (2018), which suggest that early detection of the Dengue fever and proper medical care decrease mortality rate for disease below $1 \%$ (WHO, 2018).

Figures 5.1a) to 5.1i) show the solutions of the system at the symmetric case, for the parameter values on the table (3.2) for different values of $\phi$ and for $\mu_{1}=0.009$ and $\mu_{2}=0.01$.

Figures 5.2a to $5.2 \mathrm{k}$ ) show the solutions of the system at the asymmetric case, with $\beta_{1}=45$, for the parameter values on the table 3.2 for different values of $\phi$ and for $\mu_{1}=0.009$ and $\mu_{2}=0.01$.

Figures 5.3a) to [5.3j] show the solutions of the system at the asymmetric case, with $\beta_{1}=120$, for the parameter values on the table 3.2 for different values of $\phi$ and for $\mu_{1}=0.009$ and $\mu_{2}=0.01$.

The solutions verify the theoretical analysis in previous section. Additionally, it is shown that the original system can be a good representation for the study of the propagation of the disease, even without the mortality term, hence the solutions of the new system hold similar qualitative behaviour. 


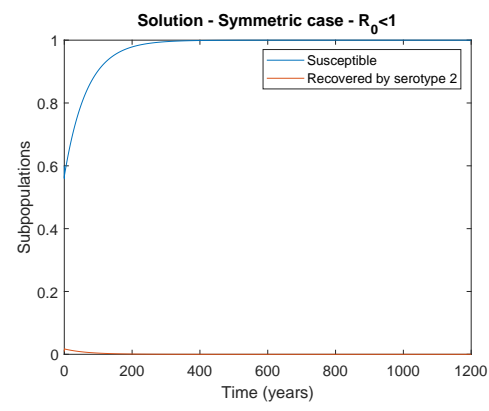

(a) $\phi=1$

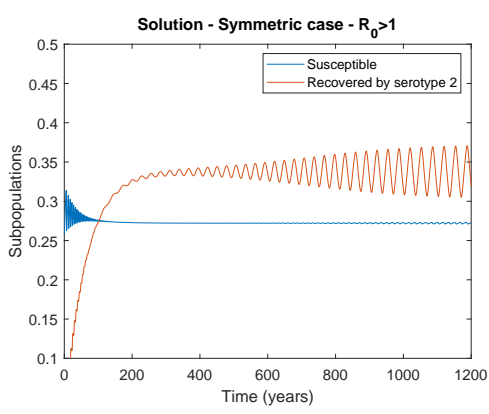

(d) $\phi=0.05$

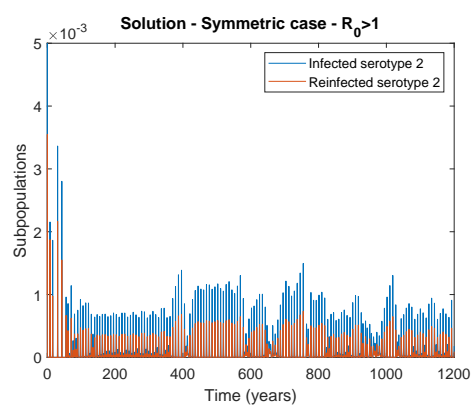

(g) $\phi=0.5$

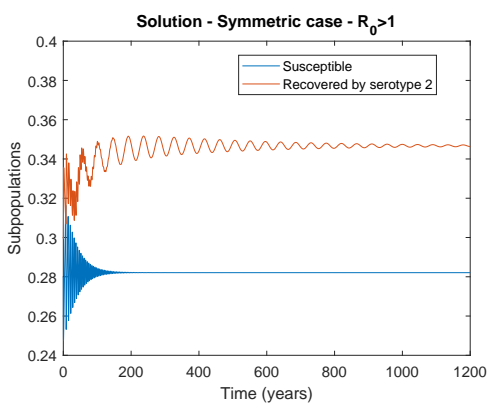

(b) $\phi=0.02$

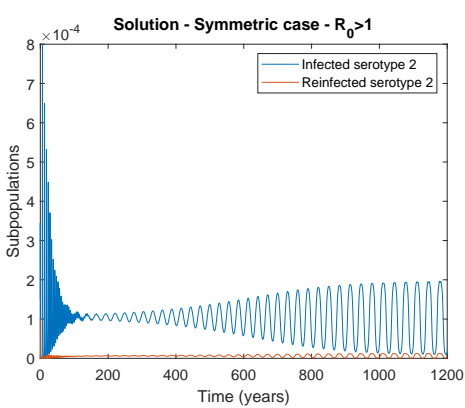

(e) $\phi=0.05$

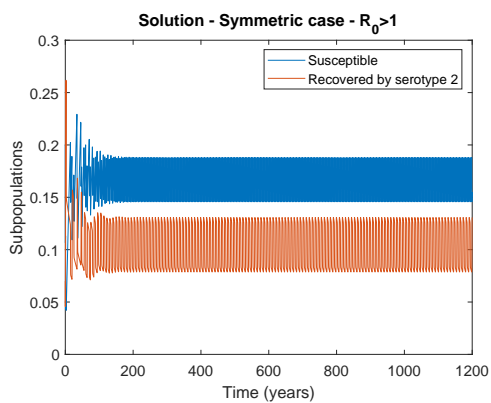

(h) $\phi=1.2$

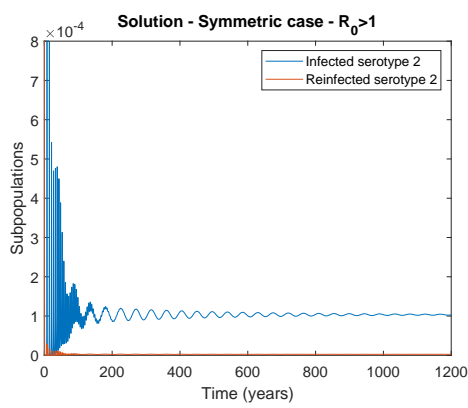

(c) $\phi=0.02$

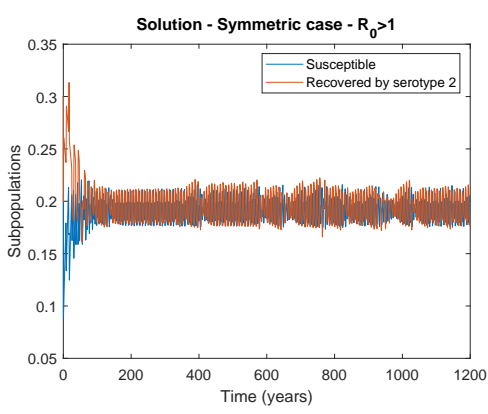

(f) $\phi=0.5$

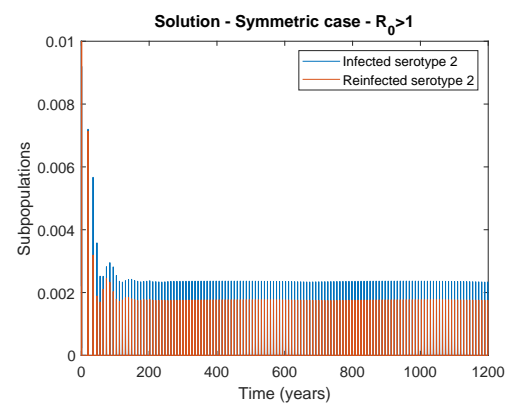

(i) $\phi=1.2$

Figure 5.1: Solutions of the system for the symmetric case. Initial conditions are found, for each value of $\phi$, in the solutions at Chapter 3 . 


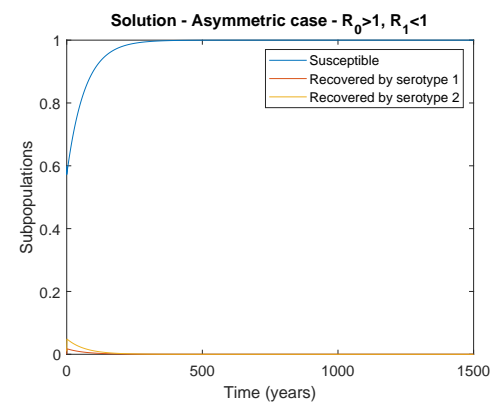

(a) $\phi=1$

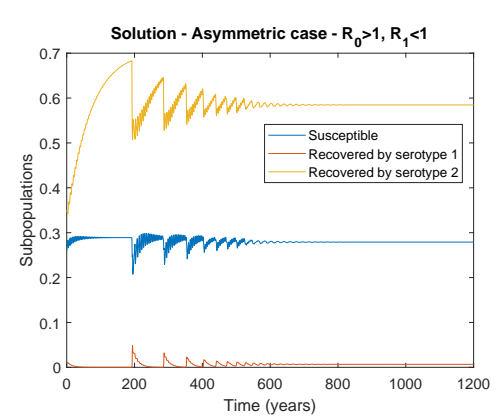

(d) $\phi=1.5$

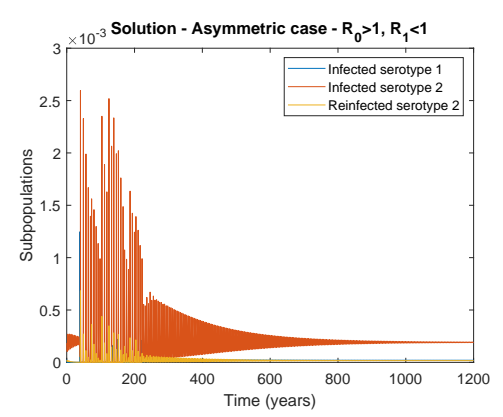

(g) $\phi=2.1$

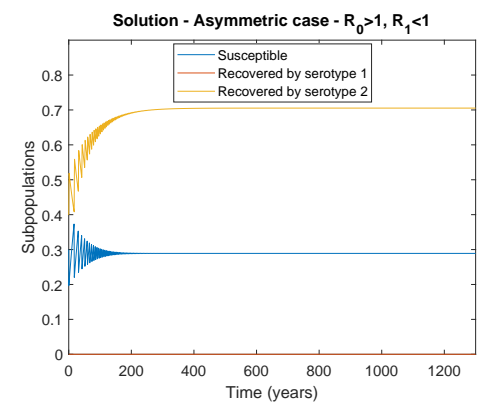

(b) $\phi=0.5$

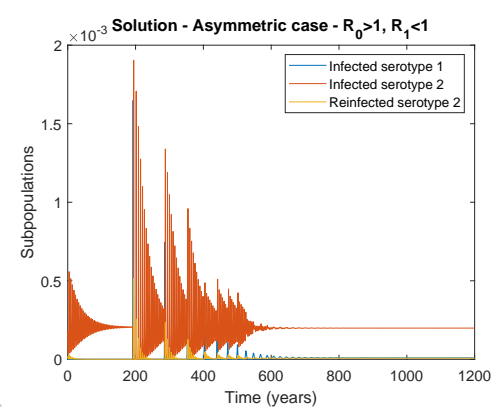

(e) $\phi=1.5$

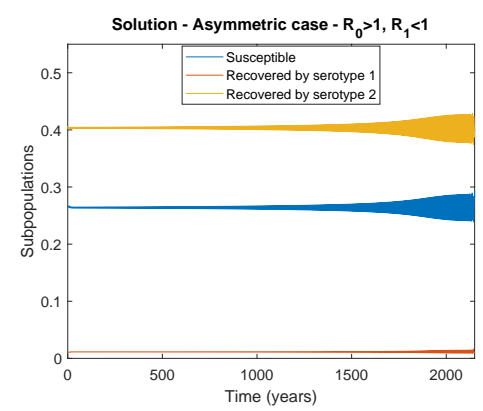

(h) $\phi=2.21$

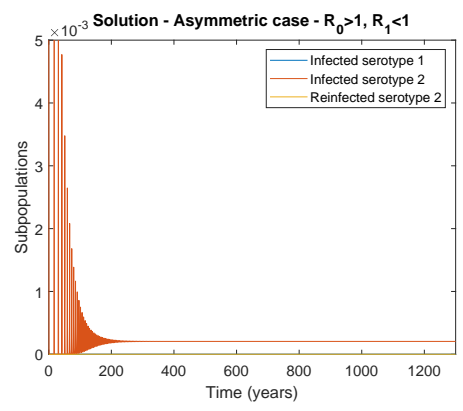

(c) $\phi=0.5$

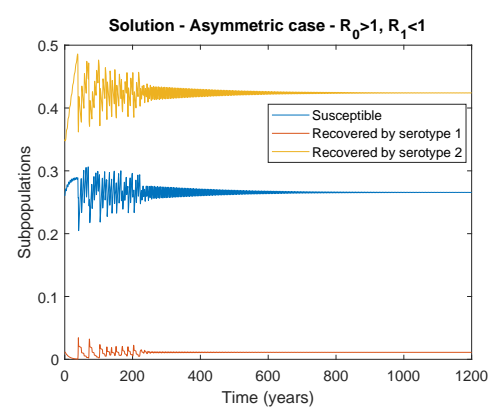

(f) $\phi=2.1$

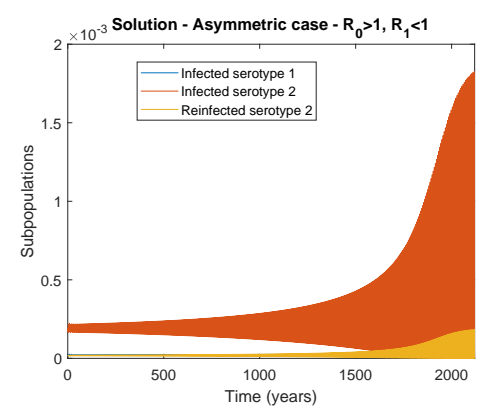

(i) $\phi=2.21$

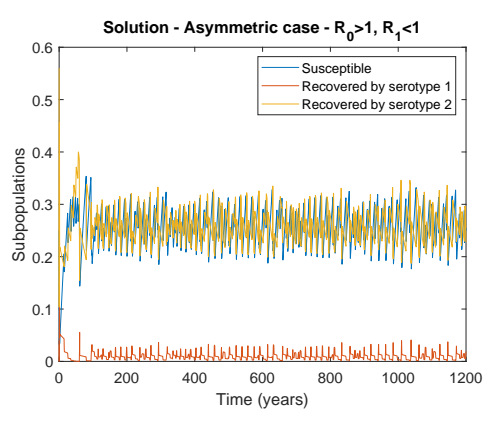

(j) $\phi=3.5$

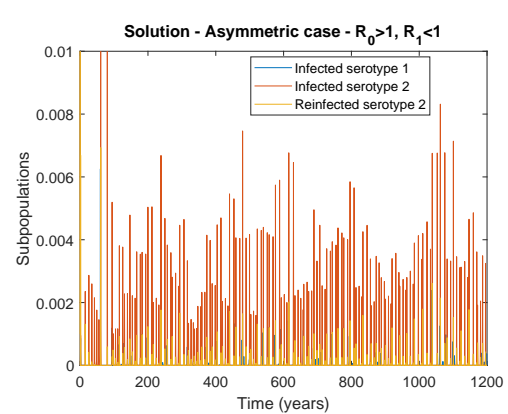

(k) $\phi=3.5$

Figure 5.2: Solutions of the system for the asymmetric case, with $\beta_{1}=45$. Initial conditions are found, for each value of $\phi$, in the solutions at Chapter 3 . 


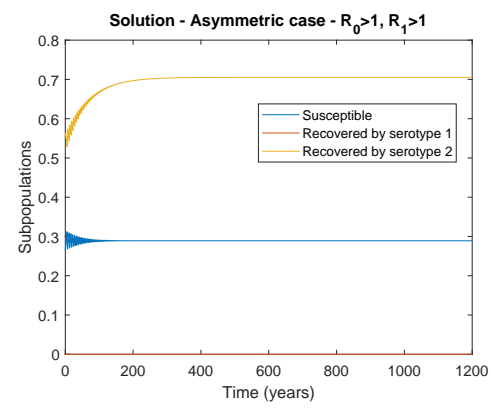

(a) $\phi=0.1$

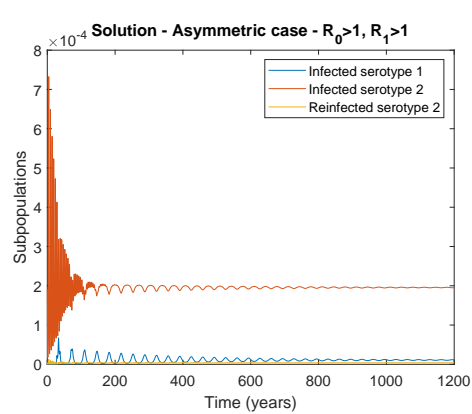

(d) $\phi=0.23$

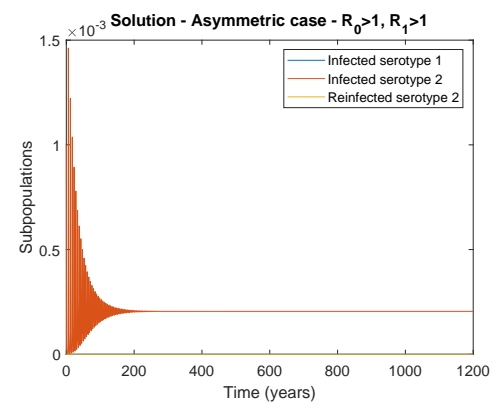

(b) $\phi=0.1$

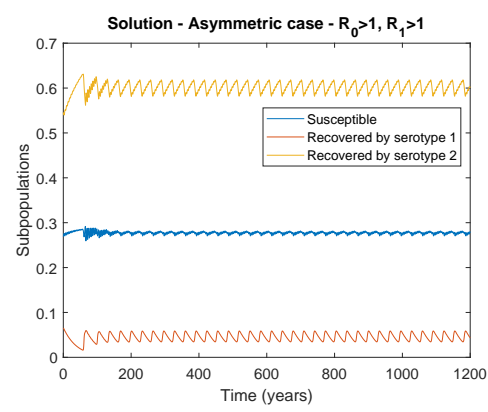

(e) $\phi=0.26$

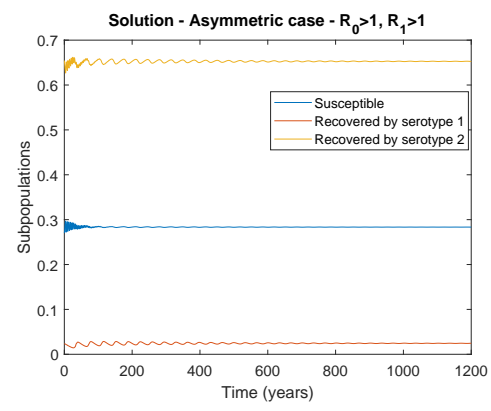

(c) $\phi=0.23$

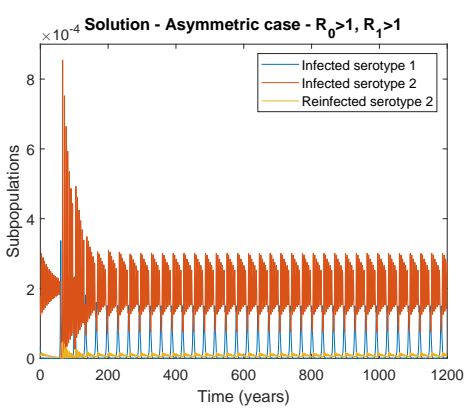

(f) $\phi=0.26$

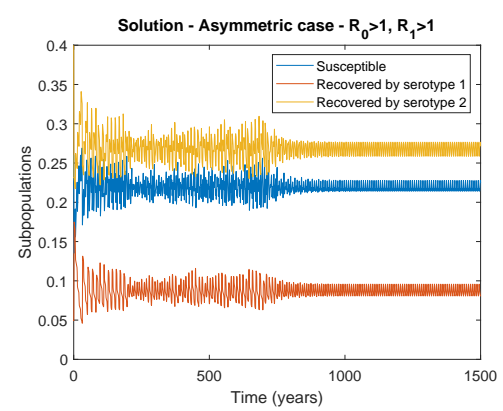

(g) $\phi=0.8$

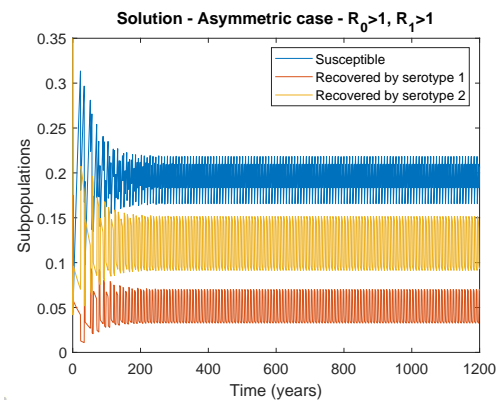

(i) $\phi=2$

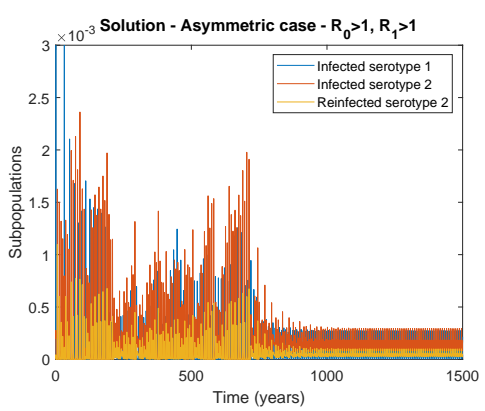

(h) $\phi=0.8$

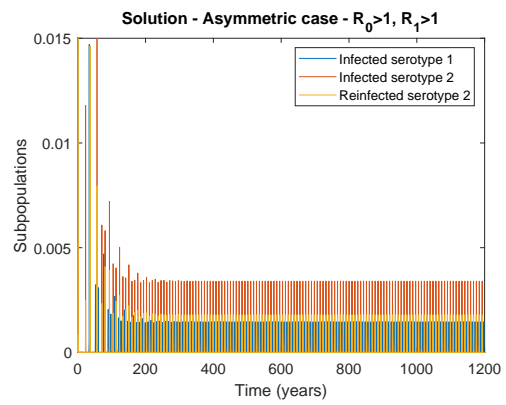

(j) $\phi=2$

Figure 5.3: Solutions of the system for the asymmetric case, with $\beta_{1}=120$. Initial conditions are found, for each value of $\phi$, in the solutions at Chapter 3 . 


\section{CHAPTER 6}

\section{Particular case}

Along chapter 2, we have proposed a model to study the spread of Dengue fever. Furthermore, we have analysed the model as we proposed with a general time of cross immunity protection, Now, we are going to analyse a particular case when the time immunity protection is exponentially distributed.

\subsection{ODE System Analysis: Equilibriums of the System}

In this section, we are going to analyse the particular case when we assume that the average cross immunity time is a constant rate being $\frac{1}{\omega_{i}}$ and the IDE system 1.7 can be described by the ODE system (1.8).

Observe that the equation that describes the dynamics for individuals in the recovered class is decoupled, hence we can establish

$$
R(t)=N(t)-S(t)-I_{1}(t)-I_{2}(t)-C_{1}(t)-C_{2}(t)-R_{1}(t)-R_{2}(t)-I_{12}(t)-I_{21}(t) .
$$

Also, the equation which describes the dynamics of the total population in time does not depend on the disease state of the individual, in other words, the disease does not interfere on the dynamic of total population. Thus, when we search for the steady state of the equation,

$$
\frac{d N(t)}{d t}=r N(t) e^{-k N(t)}-d N(t)
$$

it is found a unique positive equilibrium

$$
N^{*}=\frac{1}{k} \ln \left(\frac{r}{d}\right), \quad r>d .
$$


Consequently, we assume from now on that the size of population $N(t)$ is constant in time, namely, the total population reaches the equilibrium $N^{*}$, we look for the steady state of the system 1.8 . We search for constants functions $S^{*}, I_{1}^{*}, I_{2}^{*}, C_{1}^{*}, C_{2}^{*}, R_{1}^{*}, R_{2}^{*}, I_{12}^{*}, I_{21}^{*}$, that satisfies:

$$
\begin{aligned}
& 0=N^{*} d-d S^{*}-\overline{\beta_{1}} S^{*}\left(I_{1}^{*}+I_{21}^{*}\right)-\overline{\beta_{2}} S^{*}\left(I_{2}^{*}+I_{12}^{*}\right) \\
& 0=-(d+\gamma) I_{1}^{*}+\overline{\beta_{1}} S^{*} I_{1}^{*}+\overline{\beta_{1}} S^{*} I_{21}^{*} \\
& 0=-(d+\gamma) I_{2}^{*}+\overline{\beta_{2}} S^{*} I_{2}^{*}+\overline{\beta_{2}} S^{*} I_{12}^{*} \\
& 0=\gamma I_{1}^{*}-\left(d+\omega_{1}\right) C_{1}^{*} \\
& 0=\gamma I_{2}^{*}-\left(d+\omega_{2}\right) C_{2}^{*} \\
& 0=-d R_{1}^{*}-\overline{\alpha_{2}} \phi R_{1}^{*}\left(I_{12}^{*}+I_{2}^{*}\right)+\omega_{1} C_{1}^{*} \\
& 0=-d R_{2}^{*}-\overline{\alpha_{1}} \phi R_{2}^{*}\left(I_{21}^{*}+I_{1}^{*}\right)+\omega_{2} C_{2}^{*} \\
& 0=-(d+\gamma) I_{12}^{*}+\overline{\alpha_{2}} \phi R_{1}^{*}\left(I_{2}^{*}+I_{12}^{*}\right) \\
& 0=-(d+\gamma) I_{21}^{*}+\overline{\alpha_{1}} \phi R_{2}^{*}\left(I_{1}^{*}+I_{21}^{*}\right)
\end{aligned}
$$

where $\overline{\beta_{i}}=\frac{\beta_{i}}{N^{*}}$ and $\overline{\alpha_{i}}=\frac{\alpha_{i}}{N^{*}}$.

Clearly, the system (1.8) always has a disease-free equilibrium, namely,

$$
E_{0}=\left(N^{*}, 0,0,0,0,0,0,0,0,0\right)
$$

where $R^{*}=N^{*}-S^{*}-I_{1}^{*}-I_{2}^{*}-C_{1}^{*}-C_{2}^{*}-R_{1}^{*}-R_{2}^{*}-I_{12}^{*}-I_{21}^{*}$.

In the case of the extinction of one of the infection forces we are able to find the boundary equilibrium of the system

$$
E_{1}=\left(\frac{d+\gamma}{\overline{\beta_{1}}}, \frac{d}{\overline{\beta_{1}}}\left[\frac{\overline{\beta_{1}} N^{*}}{d+\gamma}-1\right], 0, \frac{\gamma}{d+\omega_{1}} I_{1}^{*}, 0, \frac{\omega_{1}}{d} \frac{\gamma}{d+\omega_{1}} I_{1}^{*}, 0,0,0,0\right)
$$

And, the boundary equilibrium

$$
E_{2}=\left(\frac{d+\gamma}{\overline{\beta_{2}}}, 0, \frac{d}{\overline{\beta_{2}}}\left[\frac{\overline{\beta_{2}} N^{*}}{d+\gamma}-1\right], 0, \frac{\gamma}{d+\omega_{2}} I_{2}^{*}, 0, \frac{\omega_{2}}{d} \frac{\gamma}{d+\omega_{2}} I_{2}^{*}, 0,0,0\right) .
$$

For biological reasons, we are looking for steady states which belong to $\Omega$ positively invariant region, where $\Omega=\left\{\left(S, I_{1}, I_{2}, C_{1}, C_{2}, R_{1}, R_{2}, I_{12}, I_{21}, R\right) \in \mathbb{R}_{+}^{10}\right.$ such that $S+I_{1}+I_{2}+C_{1}+C_{2}+$ $\left.R_{1}+R_{2}+I_{12}+I_{21}+R \leq N^{*}\right\}$. This way, the boundary equilibrium $E_{i}, i=1,2$ is in $\Omega$ as long as the parameters satisfy $\frac{N^{*} \overline{\overline{\beta_{i}}}}{d+\gamma}>1, i=1,2$. 
In the case of the coexistence of the two strains, we are able to find the equilibrium which is given by

$$
\begin{aligned}
C_{1}^{*} & =\frac{\gamma}{d+\omega_{1}} I_{1}^{*} \\
C_{2}^{*} & =\frac{\gamma}{d+\omega_{2}} I_{2}^{*} \\
R_{1}^{*} & =\frac{d+\gamma-\overline{\beta_{2}} S^{*}}{\overline{\alpha_{2}} \phi} \\
R_{2}^{*} & =\frac{d+\gamma-\overline{\beta_{1}} S^{*}}{\overline{\alpha_{1}} \phi} \\
I_{12}^{*} & =\frac{(d+\gamma) I_{2}^{*}-\overline{\beta_{2}} S^{*} I_{2}^{*}}{\overline{\beta_{2}} S^{*}} \\
I_{21}^{*} & =\frac{(d+\gamma) I_{1}^{*}-\overline{\beta_{1}} S^{*} I_{1}^{*}}{\overline{\beta_{1}} S^{*}} \\
I_{1}^{*}+I_{2}^{*} & =\frac{d\left(N^{*}-S^{*}\right)}{d+\gamma} \\
R^{*} & =\frac{d}{\gamma}\left(I_{12}^{*}+I_{21}^{*}\right)
\end{aligned}
$$

and, $S^{*}$ is the root of the cubic polynomial $O(S)=b_{3} S^{3}+b_{2} S^{2}+b_{1} S+b_{0}$ where

$$
\begin{aligned}
b_{3} & =\overline{\beta_{1} \beta_{2}}\left[\overline{\alpha_{2}}\left(\overline{\beta_{1}}-\overline{\alpha_{1}} \phi\right)(d+\gamma)\left(d+\omega_{2}\right)\left(\left(d+\omega_{1}\right)(d+\gamma)-\gamma \omega_{1}\right)\right. \\
& \left.+\overline{\alpha_{1}}\left(\overline{\beta_{2}}(d+\gamma)\left(d+\omega_{1}\right)-\overline{\alpha_{2}} \phi \gamma \omega_{1}\right)\left(\left(d+\omega_{2}\right)(d+\gamma)-\gamma \omega_{2}\right)\right] \\
b_{2} & =(d+\gamma)^{3}\left(d+\omega_{1}\right)\left(d+\omega_{2}\right)\left[\overline{\beta_{1}} \overline{\alpha_{2}}\left(-\overline{\beta_{1}}+\overline{\alpha_{1}} \phi\right)+\overline{\beta_{2}} \overline{\alpha_{2}}\left(-\overline{\beta_{1}}+\overline{\alpha_{1}} \phi\right)-\overline{\beta_{2}} \overline{\alpha_{1}}\left(\overline{\beta_{1}}+\overline{\beta_{2}}\right)\right] \\
& +(d+\gamma)^{2} \overline{\beta_{1} \beta_{2}}\left(\left(d+\omega_{2}\right) \gamma \omega_{1} \overline{\alpha_{2}}+\left(d+\omega_{1}\right) \gamma \omega_{2} \overline{\alpha_{1}}\right) \\
& +\overline{\beta_{1} \beta_{2}} \overline{\alpha_{1} \alpha_{2}} \phi N^{*}\left[(d+\gamma)^{2}\left(d+\omega_{1}\right)\left(d+\omega_{2}\right)-\omega_{2} \gamma^{2} \omega_{1}\right] \\
b_{1} & =(d+\gamma)^{3}\left(d+\omega_{1}\right)\left(d+\omega_{2}\right)\left[(d+\gamma)\left(\overline{\beta_{2}} \overline{\alpha_{1}}+\overline{\beta_{1}} \overline{\alpha_{2}}-\overline{\alpha_{2} \alpha_{1}} \phi\right)-N^{*} \overline{\alpha_{1} \alpha_{2}} \phi\left(\overline{\beta_{1}}+\overline{\beta_{2}}\right)\right] \\
b_{0} & =N^{*} \phi \overline{\alpha_{1} \alpha_{2}}(d+\gamma)^{4}\left(d+\omega_{1}\right)\left(d+\omega_{2}\right) .
\end{aligned}
$$

Moreover, $S^{*}$ has to satisfy that $S^{*}<\frac{d+\gamma}{\overline{\beta_{i}}}, i=1,2$, in order to have the equilibrium in the $\Omega$ region. Otherwise, if $S^{*}$ does not satisfy the inequality, the variables which represent recovered and infected population will be negative. Now, we are ready to prove the following theorems about the equilibriums of the system (1.8).

Theorem 10. If $\frac{N^{*} \overline{\beta_{1}}}{d+\gamma}>1$ then the system of equations 1.8 always has the boundary equilibrium, $E_{1}$, in $\Omega$, where

$$
E_{1}=\left(\frac{d+\gamma}{\overline{\beta_{1}}}, \frac{d}{\overline{\beta_{1}}}\left[\frac{\overline{\beta_{1}} N^{*}}{d+\gamma}-1\right], 0, \frac{\gamma}{d+\omega_{1}} I_{1}^{*}, 0, \frac{\omega_{1}}{d} \frac{\gamma}{d+\omega_{1}} I_{1}^{*}, 0,0,0,0\right)
$$


And, if $\frac{N^{*} \overline{\beta_{2}}}{d+\gamma}>1$ then the system of equations 1.8 always has the boundary equilibrium, $E_{2}$, in $\Omega$, where

$$
E_{2}=\left(\frac{d+\gamma}{\overline{\beta_{2}}}, 0, \frac{d}{\overline{\beta_{2}}}\left[\frac{\overline{\beta_{2}} N^{*}}{d+\gamma}-1\right], 0, \frac{\gamma}{d+\omega_{2}} I_{2}^{*}, 0, \frac{\omega_{2}}{d} \frac{\gamma}{d+\omega_{2}} I_{2}^{*}, 0,0,0\right) .
$$

Proof. It is easy to see that, if $\frac{N^{*} \overline{\beta_{i}}}{d+\gamma}>1$, for $i=1,2$ then $I_{i}^{*}, C_{i}^{*}$ and $R_{i}^{*}$, for $i=1,2$ are positive and, the total sum is $N^{*}$. Thus $E_{i}$ is in $\Omega$, for $i=1,2$ and the system has always a boundary equilibrium with such condition.

Theorem 11. Without loss of generality, we suppose that $\bar{\beta}_{2}>\overline{\beta_{1}}$. If $\max \left\{\frac{N^{*} \overline{\beta_{1}}}{d+\gamma}, \frac{N^{*} \overline{\beta_{2}}}{d+\gamma}\right\}>1$ and,

$$
L=\frac{\overline{\beta_{1}}}{\overline{\beta_{2}}}+\left(\frac{N^{*} \overline{\beta_{2}}}{d+\gamma}-1\right) \frac{\overline{\alpha_{1}} \phi \gamma \omega_{2}}{\overline{\beta_{2}}\left(d+\omega_{2}\right)(d+\gamma)}>1
$$

then, the system (1.8) admits an interior equilibrium in $\Omega$ with the coexistence of the two strains.

Proof. The independent term, $b_{0}$, of the polynomial $O(S)$ is always positive. Since the equilibrium is given by (6.3) with $S^{*}$ being a root of the polynomial $O$ and, this equilibrium will be in the region $\Omega$ if $S^{*}<\frac{d+\gamma}{\beta_{i}}$, for $i=1,2$, we define

$$
S_{\text {min }}=\min \left\{\frac{d+\gamma}{\overline{\beta_{1}}}, \frac{d+\gamma}{\overline{\beta_{2}}}\right\}=\frac{d+\gamma}{\overline{\beta_{2}}} .
$$

Then, if $\frac{N^{*} \overline{\beta_{2}}}{d+\gamma}>1$ and $L>1$, the polynomial $O$ at $S_{\min }$ is

$$
O\left(S_{\text {min }}\right)=\frac{\left[(d+\gamma)^{2} \gamma \omega_{1} \overline{\alpha_{2}} \overline{\beta_{1}}\right]}{{\overline{\beta_{2}}}^{2}}\left[\left(d+\omega_{2}\right)(d+\gamma)^{2}\left(\overline{\beta_{2}}-\overline{\beta_{1}}\right)+\gamma \omega_{2} \overline{\alpha_{1}} \phi\left((d+\gamma)-N^{*} \overline{\beta_{2}}\right)\right]<0 .
$$

This shows that we have a root $S^{*}$ of the polynomial $O$, such that, $0<S^{*}<S_{\min }$. Therefore, for this $S^{*}$ the positive equilibrium $E_{3}$ with the variables satisfying $[6.3$ ) is $\Omega$. And, this shows that we have a positive equilibrium of the system 1.8 , in $\Omega$, with the coexistence of the two strains.

\subsubsection{Symmetric Case}

Once we are restricted to the case $\overline{\beta_{1}}=\overline{\beta_{2}}=\beta, \overline{\alpha_{1}}=\overline{\alpha_{2}}=\alpha$ and, $\omega_{1}=\omega_{2}=\omega$, we can write the cubic polynomial $O(S)$ as $O(S)=Q(S)(b S+a)$ where

$$
\begin{aligned}
& a=-\alpha \phi(d+\gamma)^{2}(d+\omega) \\
& b=\alpha \phi \beta((d+\gamma)(d+\omega)-\gamma \omega)
\end{aligned}
$$


and, $Q(S)=a_{2} S^{2}+a_{1} S+a_{0}$ where

$$
\begin{aligned}
& a_{2}=\beta[(d+\gamma)(\alpha \phi-2 \beta)(d+\omega)+\gamma \omega \alpha \phi] \\
& a_{1}=(d+\gamma)^{2}(2 \beta-\alpha \phi)(d+\omega)-N^{*} \alpha \phi \beta((d+\gamma)(d+\omega)+\gamma \omega) \\
& a_{0}=N^{*} \alpha \phi(d+\gamma)^{2}(d+\omega) .
\end{aligned}
$$

Since $S^{*}=\frac{-a}{b}=\frac{(d+\gamma)^{2}(d+\omega)}{\beta((d+\gamma)(d+\omega)-\gamma \omega)}$ gives us a negative value for $I_{12}^{*}$ and $I_{21}^{*}$, it means that the equilibrium will not be in the $\Omega$ region, as a consequence we look for the $Q(S)$ roots.

Searching for the roots, we need to remember that we want a $S^{*}$, root of $Q(S)$ polynomial, which is positive and, $S^{*}<\frac{d+\gamma}{\beta}$, in order to have an equilibrium in the $\Omega$ region. This gives the proof of the following theorem.

Theorem 12. If $\frac{N^{*} \beta}{d+\gamma}>1$ then the system of equations $(1.8)$, restricted to the case $\overline{\beta_{1}}=\overline{\beta_{2}}=\beta$, $\overline{\alpha_{1}}=\overline{\alpha_{2}}=\alpha$ and, $\omega_{1}=\omega_{2}=\omega$, always has two boundary equilibriums in $\Omega$, namely,

$$
\begin{aligned}
& E_{1}=\left(\frac{d+\gamma}{\beta}, \frac{d}{\beta}\left[\frac{\beta N^{*}}{d+\gamma}-1\right], 0, \frac{\gamma}{d+\omega} I_{1}^{*}, 0, \frac{\omega}{d} \frac{\gamma}{d+\omega} I_{1}^{*}, 0,0,0,0\right), \\
& E_{2}=\left(\frac{d+\gamma}{\beta}, 0, \frac{d}{\beta}\left[\frac{\beta N^{*}}{d+\gamma}-1\right], 0, \frac{\gamma}{d+\omega} I_{2}^{*}, 0, \frac{\omega}{d} \frac{\gamma}{d+\omega} I_{2}^{*}, 0,0,0\right)
\end{aligned}
$$

and a unique positive equilibrium in $\Omega$, with coexistence of the two strains, where

$$
\begin{aligned}
S^{*} & =\frac{(d+\gamma)^{2}(-2 \beta+\alpha \phi)(d+\omega)+N^{*} \alpha \phi \beta((d+\gamma)(d+\omega)+\gamma \omega)}{2 \beta[(d+\gamma)(\alpha \phi-2 \beta)(d+\omega)+\gamma \omega \alpha \phi]} \\
& -\frac{\sqrt{\left((d+\gamma)^{2}(d+\omega)(2 \beta-\alpha \phi)+N^{*} \alpha \phi \beta(\gamma \omega+(d+\gamma)(d+\omega))\right)^{2}-8 N^{*} \alpha \phi \beta^{2} \gamma \omega(d+\gamma)^{2}(d+\omega)}}{2 \beta[(d+\gamma)(\alpha \phi-2 \beta)(d+\omega)+\gamma \omega \alpha \phi]}
\end{aligned}
$$

and, $I_{1}^{*}=I_{2}^{*}=\frac{d}{2(d+\gamma)}\left(N-S^{*}\right), \quad C_{1}^{*}=C_{2}^{*}, \quad R_{1}^{*}=R_{2}^{*}, \quad I_{12}^{*}=I_{21}^{*}$ satisfies 6.3.

Proof. If $\frac{N^{*} \beta}{d+\gamma}>1$ then, it is easy to see that $E_{i}$ is in $\Omega$, for $i=1,2$.

In addition, since the searched root $S^{*}$ needs to be smaller than $\frac{d+\gamma}{\beta}$, we define

$$
S_{\max }=\frac{d+\gamma}{\beta} .
$$

Then, the quadratic polynomial evaluated in $S_{\max }$ will be

$$
Q\left(S_{\max }\right)=Q\left(\frac{d+\gamma}{\beta}\right)=(d+\gamma) \omega \gamma \alpha \phi\left(\frac{d+\gamma}{\beta}-N^{*}\right)<0
$$

because $\frac{N^{*} \beta}{d+\gamma}>1$. 
Besides, the independent term $a_{0}$ of the polynomial $Q(S)$ polynomial is positive. This proves that we have a positive root satisfying $S^{*}<\frac{d+\gamma}{\beta}$, and it is given by

$$
\begin{aligned}
S^{*} & =\frac{(d+\gamma)^{2}(-2 \beta+\alpha \phi)(d+\omega)+N^{*} \alpha \phi \beta((d+\gamma)(d+\omega)+\gamma \omega)}{2 \beta[(d+\gamma)(\alpha \phi-2 \beta)(d+\omega)+\gamma \omega \alpha \phi]} \\
& -\frac{\sqrt{\left((d+\gamma)^{2}(d+\omega)(2 \beta-\alpha \phi)+N^{*} \alpha \phi \beta(\gamma \omega+(d+\gamma)(d+\omega))\right)^{2}-8 N^{*} \alpha \phi \beta^{2} \gamma \omega(d+\gamma)^{2}(d+\omega)}}{2 \beta[(d+\gamma)(\alpha \phi-2 \beta)(d+\omega)+\gamma \omega \alpha \phi]}
\end{aligned}
$$

Therefore, the equilibrium $E_{3}=\left(S^{*}, I_{1}^{*}, I_{2}^{*}, C_{1}^{*}, C_{2}^{*}, R_{1}^{*}, R_{2}^{*}, I_{12}^{*}, I_{21}^{*}, R^{*}\right)$ is in $\Omega$, where $S^{*}$ is given by 6.6 and, from 6.3,

$$
\begin{aligned}
& I_{1}^{*}=I_{2}^{*}=\frac{d\left(N^{*}-S^{*}\right)}{2(d+\gamma)}, \\
& C_{1}^{*}=C_{2}^{*}=\frac{\gamma}{d+\omega} I_{1}^{*}, \\
& R_{1}^{*}=R_{2}^{*}=\frac{d+\gamma-\beta S^{*}}{\alpha \phi}, \\
& I_{12}^{*}=I_{21}^{*}=\frac{(d+\gamma) I_{1}^{*}-\beta S^{*} I_{1}^{*}}{\beta S^{*}}, \\
& R^{*}=N^{*}-S^{*}-I_{1}^{*}-I_{2}^{*}-C_{1}^{*}-C_{2}^{*}-R_{1}^{*}-R_{1}^{*}-I_{12}^{*}-I_{21}^{*} .
\end{aligned}
$$

\subsection{Stability Analysis of the Equilibriums}

\subsubsection{Basic Reproduction Number}

The Basic Reproduction Number $\mathcal{R}_{0}$ is defined by many authors, such as Van den Driessche (2008) as the expected number of secondary infections produced by one case in a susceptible population and, also as a measure of the potential for disease spread in a population. Mathematically, the Basic Reproduction Number is a threshold for stability of Disease Free equilibrium (Van den Driessche, 2008).

Since the values $\frac{N^{*} \overline{\beta_{1}}}{d+\gamma}$ and $\frac{N^{*} \overline{\beta_{2}}}{d+\gamma}$ have appeared many times as a threshold value for the boundary equilibriums to be in $\Omega$ region and, also, as a threshold for the stability of Disease Free equilibrium, we will define it as the Basic Reproduction Number.

As well as the studies by Van den Driessche (2008) and Kooi (2014) we will define the threshold

$$
\mathcal{R}_{0}=\frac{N^{*} \beta}{d+\gamma}
$$


as the Basic Reproduction Number of the system for symmetric case, when the parameters are equal.

And, the threshold values

$$
\mathcal{R}_{1}=\frac{N^{*} \overline{\beta_{1}}}{d+\gamma} \text { and } \mathcal{R}_{2}=\frac{N^{*} \overline{\beta_{2}}}{d+\gamma}
$$

as the Basic Reproduction Number, for asymmetric case, for infection one and two, respectively.

It is usual, in this case, when it is a multi-strain model with different strains, to define an overall Reproduction Number for the system. Thus, for asymmetric case, the Basic Reproduction Number for the system will be defined as

$$
\mathcal{R}_{0}=\max \left\{\mathcal{R}_{1}, \mathcal{R}_{2}\right\}
$$

According to Martcheva (2015), this definition is related to the one found in Van den Driessche (2008), where the Basic Reproduction Number is defined mathematically as the spectral radius (the maximum of the modulus of the eigenvalues) of the Next Generation matrix.

\subsubsection{Local Stability}

The local stability of the equilibriums will be determined by the classical method of determining stability of the steady states of some ODE system, by analysis of the eigenvalues of the Jacobian matrix of the system at each equilibrium. Since the equation for $R$ is decoupled of the system (1.8) and, we can establish

$$
R(t)=N^{*}-S(t)-I_{1}(t)-I_{2}(t)-C_{1}(t)-C_{2}(t)-R_{1}(t)-R_{2}(t)-I_{12}(t)-I_{21}(t),
$$

then, it is sufficient to write the equilibriums in the form $E=\left(S^{*}, I_{1}^{*}, I_{2}^{*}, C_{1}^{*}, C_{2}^{*}, R_{1}^{*}, R_{1}^{*}, I_{12}^{*}, I_{21}^{*}\right)$ (without the last input) as well as analysing the Jacobian matrix of the system at the equilibriums in this way.

\begin{tabular}{|c|c|c|c|c|c|c|c|c|c|}
\hline & {$\left[-d-V_{0}\right.$} & $-\overline{\beta_{1}} S^{\star}$ & $-\overline{\beta_{2}} S^{\star}$ & 0 & 0 & 0 & 0 & $-\overline{\beta_{2}} S^{\star}$ & $-\overline{\beta_{1}} S^{\star}$ \\
\hline & $\overline{\beta_{1}}\left(I_{1}^{\star}+I_{21}^{\star}\right)$ & $\overline{\beta_{1}} S^{\star}-(d+\gamma)$ & 0 & 0 & 0 & 0 & 0 & 0 & $\overline{\beta_{1}} S^{\star}$ \\
\hline & $\overline{\beta_{2}}\left(I_{2}^{\star}+I_{12}^{\star}\right)$ & 0 & $\overline{\beta_{2}} S^{\star}-(d+\gamma)$ & 0 & 0 & 0 & 0 & $\overline{\beta_{2}} S^{\star}$ & 0 \\
\hline & 0 & $\gamma$ & 0 & $-\left(d+\omega_{1}\right)$ & 0 & 0 & 0 & 0 & 0 \\
\hline \multirow[t]{5}{*}{$J(E)=$} & 0 & 0 & $\gamma$ & 0 & $-\left(d+\omega_{2}\right)$ & 0 & 0 & 0 & 0 \\
\hline & 0 & 0 & $-\overline{\alpha_{2}} \phi R_{1}^{\star}$ & $\omega_{1}$ & 0 & $V_{1}$ & 0 & $-\overline{\alpha_{2}} \phi R_{1}^{\star}$ & 0 \\
\hline & 0 & $-\overline{\alpha_{1}} \phi R_{2}^{\star}$ & 0 & 0 & $\omega_{2}$ & 0 & $V_{2}$ & 0 & $-\overline{\alpha_{1}} \phi R_{2}^{\star}$ \\
\hline & 0 & 0 & $\overline{\alpha_{2}} \phi R_{1}^{\star}$ & 0 & 0 & $\overline{\alpha_{2}} \phi\left(I_{2}^{\star}+I_{12}^{\star}\right)$ & 0 & $V_{3}$ & 0 \\
\hline & 0 & $\overline{\alpha_{1}} \phi R_{2}^{\star}$ & 0 & 0 & 0 & 0 & $\overline{\alpha_{1}} \phi\left(I_{1}^{\star}+I_{21}^{\star}\right)$ & 0 & $V_{4}$ \\
\hline
\end{tabular}

The Jacobian matrix of the system at any equilibrium $E=\left(S^{\star}, I_{1}^{\star}, I_{2}^{\star}, C_{1}^{\star}, C_{2}^{\star}, R_{1}^{\star}, R_{1}^{\star}, I_{12}^{\star}, I_{21}^{\star}\right)$ of the system is given by

where $\overline{\beta_{i}}=\frac{\beta_{i}}{N^{*}}, \overline{\alpha_{i}}=\frac{\alpha_{i}}{N^{*}}$, for $i=1,2, \quad V_{0}=\overline{\beta_{1}}\left(I_{1}^{\star}+I_{21}^{\star}\right)+\overline{\beta_{2}}\left(I_{2}^{\star}+I_{12}^{\star}\right), \quad V_{1}=-\left(d+\overline{\alpha_{2}} \phi\left(I_{2}^{\star}+I_{12}^{\star}\right)\right)$, $V_{2}=-\left(d+\overline{\alpha_{1}} \phi\left(I_{1}^{\star}+I_{21}^{\star}\right)\right), \quad V_{3}=-(d+\gamma)+\overline{\alpha_{2}} \phi R_{1}^{\star}$ and, $V_{4}=-(d+\gamma)+\overline{\alpha_{1}} \phi R_{2}^{\star}$.

So, the Jacobian matrix of the system evaluated at the Disease Free equilibrium $E_{0}=\left(N^{*}, 0,0,0,0,0,0,0,0\right)$ is 


$$
J\left(E_{0}\right)=\left[\begin{array}{ccccccccc}
-d & -\overline{\beta_{1}} N^{*} & -\overline{\beta_{2}} N^{*} & 0 & 0 & 0 & 0 & -\overline{\beta_{2}} N^{*} & -\overline{\beta_{1}} N^{*} \\
0 & \overline{\beta_{1}} N^{*}-(d+\gamma) & 0 & 0 & 0 & 0 & 0 & 0 & \overline{\beta_{1}} N^{*} \\
0 & 0 & \overline{\beta_{2}} N^{*}-(d+\gamma) & 0 & 0 & 0 & 0 & \overline{\beta_{2}} N^{*} & 0 \\
0 & \gamma & 0 & -\left(d+\omega_{1}\right) & 0 & 0 & 0 & 0 & 0 \\
0 & 0 & \gamma & 0 & -\left(d+\omega_{2}\right) & 0 & 0 & 0 & 0 \\
0 & 0 & 0 & \omega_{1} & 0 & -d & 0 & 0 & 0 \\
0 & 0 & 0 & 0 & \omega_{2} & 0 & -d & 0 & 0 \\
0 & 0 & 0 & 0 & 0 & 0 & 0 & -(d+\gamma) & 0 \\
0 & 0 & 0 & 0 & 0 & 0 & 0 & 0 & -(d+\gamma)
\end{array}\right]
$$

and its eigenvalues are

$$
\begin{aligned}
& \lambda_{1}=-d \\
& \lambda_{2}=-\left(d+\omega_{1}\right) \\
& \lambda_{3}=-\left(d+\omega_{2}\right) \\
& \lambda_{4}=-(d+\gamma) \\
& \lambda_{5}=-(d+\gamma) \\
& \lambda_{6}=-d \\
& \lambda_{7}=-d \\
& \lambda_{8}=-(d+\gamma)+\overline{\beta_{1}} N^{*} \\
& \lambda_{9}=-(d+\gamma)+\overline{\beta_{2}} N^{*} .
\end{aligned}
$$

It gives us the proof of the following theorem about the stability of Disease Free Equilibrium (DFE).

Theorem 13. If $\max \left\{\mathcal{R}_{1}, \mathcal{R}_{2}\right\}<1$ then the DFE, $E_{0}=\left(N^{*}, 0,0,0,0,0,0,0,0\right)$, of the system (1.8) is locally asymptotically stable. And $E_{0}$ is unstable if $\mathcal{R}_{1}>1$ or $\mathcal{R}_{2}>1$.

Proof. If $\frac{N^{*} \overline{\beta_{1}}}{d+\gamma}<1$ and $\frac{N^{*} \overline{\beta_{2}}}{d+\gamma}<1$, all the eigenvalues of Jacobian matrix at $E_{0}$, given by 6.10 , are negative. Then $E_{0}$ is locally stable. In case of $\frac{N^{*} \overline{\beta_{1}}}{d+\gamma}>1$ or $\frac{N^{*} \overline{\beta_{2}}}{d+\gamma}>1$, we have the eigenvalues $\lambda_{8}$ or $\lambda_{9}$ positive and, therefore $E_{0}$ will have at least one unstable direction. In addition, with $\beta_{1}<\beta_{2}$ we have that $\mathcal{R}_{1}<\mathcal{R}_{2}$. Then, if $1<\mathcal{R}_{1}<\mathcal{R}_{2}$, also, $E_{0}$ will be unstable.

The stability of the Boundary equilibriums, in other words, the steady state in case of extinction of one of the strains, will be determined by the sign of the eigenvalues of the Jacobian matrix in the equilibrium.

The Jacobian matrix of the system evaluated at

$$
E_{1}=\left(\frac{d+\gamma}{\overline{\beta_{1}}}, \frac{d}{\overline{\beta_{1}}}\left(\mathcal{R}_{1}-1\right), 0, \frac{\gamma}{d+\omega_{1}} I_{1}^{*}, 0, \frac{\omega_{1}}{d} \frac{\gamma}{d+\omega_{1}} I_{1}^{*}, 0,0,0\right)
$$


is given by

$$
J\left(E_{1}\right)=\left[\begin{array}{ccccccccc}
-d \mathcal{R}_{1} & -(d+\gamma) & -\overline{\beta_{2}} \frac{d+\gamma}{\bar{\beta}_{1}} & 0 & 0 & 0 & 0 & -\overline{\beta_{2}} \frac{d+\gamma}{\overline{\beta_{1}}} & -(d+\gamma) \\
d\left(\mathcal{R}_{1}-1\right) & 0 & 0 & 0 & 0 & 0 & 0 & 0 & (d+\gamma) \\
0 & 0 & (d+\gamma)\left(\frac{\overline{\beta_{2}}}{\beta_{1}}-1\right) & 0 & 0 & 0 & 0 & \overline{\beta_{2}} \frac{d+\gamma}{\overline{\beta_{1}}} & 0 \\
0 & \gamma & 0 & -\left(d+\omega_{1}\right) & 0 & 0 & 0 & 0 & 0 \\
0 & 0 & \gamma & 0 & -\left(d+\omega_{2}\right) & 0 & 0 & 0 & 0 \\
0 & 0 & -W_{1}\left(\mathcal{R}_{1}-1\right) & \omega_{1} & 0 & -d & 0 & -W_{1}\left(\mathcal{R}_{1}-1\right) & 0 \\
0 & 0 & 0 & 0 & \omega_{2} & 0 & -d-W_{3} & 0 & 0 \\
0 & 0 & W_{1}\left(\mathcal{R}_{1}-1\right) & 0 & 0 & 0 & 0 & W_{2} & 0 \\
0 & 0 & 0 & 0 & 0 & 0 & W_{3} & 0 & -(d+\gamma)
\end{array}\right],
$$

where $W_{1}=\frac{\overline{\alpha_{2}} \phi}{\overline{\beta_{1}}} \frac{\gamma \omega_{1}}{d+\omega_{1}}, W_{2}=-(d+\gamma)+W_{1}\left(\mathcal{R}_{1}-1\right)$ and, $W_{3}=\frac{\overline{\alpha_{1}} \phi}{\overline{\beta_{1}}} d\left(\mathcal{R}_{1}-1\right)$. And its eigenvalues are

$$
\begin{aligned}
& \lambda_{1}=-d \\
& \lambda_{2}=-\left(d+\omega_{1}\right) \\
& \lambda_{3}=-\left(d+\omega_{2}\right) \\
& \lambda_{4}=-(d+\gamma) \\
& \lambda_{5}=-(d+\gamma) \\
& \lambda_{6}=-d\left(1+\frac{\overline{\alpha_{1}} \phi}{\overline{\beta_{1}}}\left(\mathcal{R}_{1}-1\right)\right) \\
& \lambda_{7}=\frac{1}{2}\left(-d \mathcal{R}_{1}-\sqrt{\left(d \mathcal{R}_{1}\right)^{2}-4 d(d+\gamma)\left(\mathcal{R}_{1}-1\right)}\right) \\
& \lambda_{8}=\frac{1}{2}\left(-d \mathcal{R}_{1}+\sqrt{\left(d \mathcal{R}_{1}\right)^{2}-4 d(d+\gamma)\left(\mathcal{R}_{1}-1\right)}\right) \\
& \lambda_{9}=\frac{\overline{\alpha_{2}} \phi \gamma \omega_{1}\left(\mathcal{R}_{1}-1\right)}{\overline{\beta_{1}}\left(d+\omega_{1}\right)}+\frac{\left(\overline{\beta_{2}}-\overline{\beta_{1}}\right)(d+\gamma)\left(d+\omega_{1}\right)}{\overline{\beta_{1}}\left(d+\omega_{1}\right)} .
\end{aligned}
$$

While, the Jacobian matrix of the system evaluated at

$$
E_{2}=\left(\frac{d+\gamma}{\overline{\beta_{2}}}, 0, \frac{d}{\overline{\beta_{2}}}\left(\mathcal{R}_{2}-1\right), 0, \frac{\gamma}{d+\omega_{2}} I_{2}^{*}, 0, \frac{\omega_{2}}{d} \frac{\gamma}{d+\omega_{2}} I_{2}^{*}, 0,0\right)
$$

is given by

$$
J\left(E_{2}\right)=\left[\begin{array}{ccccccccc}
-d \mathcal{R}_{2} & -\overline{\beta_{1}} \frac{d+\gamma}{\overline{\beta_{2}}} & -(d+\gamma) & 0 & 0 & 0 & 0 & -(d+\gamma) & -\overline{\beta_{1}} \frac{d+\gamma}{\overline{\beta_{2}}} \\
0 & (d+\gamma)\left(\frac{\overline{\beta_{1}}}{\bar{\beta}_{2}}-1\right) & 0 & 0 & 0 & 0 & 0 & 0 & \overline{\beta_{1}} \frac{d+\gamma}{\bar{\beta}_{2}} \\
d\left(\mathcal{R}_{2}-1\right) & 0 & 0 & 0 & 0 & 0 & 0 & (d+\gamma) & 0 \\
0 & \gamma & 0 & -\left(d+\omega_{1}\right) & 0 & 0 & 0 & 0 & 0 \\
0 & 0 & \gamma & 0 & -\left(d+\omega_{2}\right) & 0 & 0 & 0 & 0 \\
0 & 0 & 0 & \omega_{1} & 0 & -d-Z_{1} & 0 & 0 & 0 \\
0 & -Z_{2}\left(\mathcal{R}_{2}-1\right) & 0 & 0 & \omega_{2} & 0 & -d & 0 & -Z_{2}\left(\mathcal{R}_{2}-1\right) \\
0 & 0 & 0 & 0 & 0 & Z_{1} & 0 & -(d+\gamma) & 0 \\
0 & Z_{2}\left(\mathcal{R}_{2}-1\right) & 0 & 0 & 0 & 0 & 0 & 0 & Z_{3}
\end{array}\right],
$$


where $Z_{1}=\frac{\overline{\alpha_{2}} \phi}{\overline{\beta_{2}}} d\left(\mathcal{R}_{2}-1\right), \quad Z_{2}=\frac{\overline{\alpha_{1}} \phi}{\overline{\beta_{2}}} \frac{\gamma \omega_{2}}{d+\omega_{2}}$ and, $Z_{3}=-(d+\gamma)+Z_{2}\left(\mathcal{R}_{2}-1\right)$. And its eigenvalues are

$$
\begin{aligned}
& \lambda_{1}=-d \\
& \lambda_{2}=-\left(d+\omega_{1}\right) \\
& \lambda_{3}=-\left(d+\omega_{2}\right) \\
& \lambda_{4}=-(d+\gamma) \\
& \lambda_{5}=-(d+\gamma) \\
& \lambda_{6}=-d\left(1+\frac{\overline{\alpha_{2}} \phi}{\overline{\beta_{2}}}\left(\mathcal{R}_{2}-1\right)\right) \\
& \lambda_{7}=\frac{1}{2}\left(-d \mathcal{R}_{2}-\sqrt{\left(d \mathcal{R}_{2}\right)^{2}-4 d(d+\gamma)\left(\mathcal{R}_{2}-1\right)}\right) \\
& \lambda_{8}=\frac{1}{2}\left(-d \mathcal{R}_{2}+\sqrt{\left(d \mathcal{R}_{2}\right)^{2}-4 d(d+\gamma)\left(\mathcal{R}_{2}-1\right)}\right) \\
& \lambda_{9}=\frac{\overline{\alpha_{1}} \phi \gamma \omega_{2}\left(\mathcal{R}_{2}-1\right)}{\overline{\beta_{2}}\left(d+\omega_{2}\right)}+\frac{\left(\overline{\beta_{1}}-\overline{\beta_{2}}\right)(d+\gamma)\left(d+\omega_{2}\right)}{\overline{\beta_{2}}\left(d+\omega_{2}\right)} .
\end{aligned}
$$

It gives us the following theorem about the stability of Boundary equilibriums.

Theorem 14. Without loss of generality, we assume $\overline{\beta_{2}}>\overline{\beta_{1}}$. Then, the Boundary equilibrium, $E_{1}$, given by (6.11), of the system (1.8) is always unstable, in $\Omega$ region. And, $E_{2}$ given by (6.13) is stable in $\Omega$, if $\mathcal{R}_{s}<1$ and, unstable in $\Omega$, if $\mathcal{R}_{s}>1$, where

$$
\mathcal{R}_{s}=\frac{\mathcal{R}_{1}}{\mathcal{R}_{2}}+\left(\mathcal{R}_{2}-1\right) \frac{\overline{\alpha_{1}} \phi \gamma \omega_{2}}{\overline{\beta_{2}}\left(d+\omega_{2}\right)(d+\gamma)}
$$

Proof. Since $E_{1}$ is in $\Omega$ we have that $\mathcal{R}_{1}>1$. Then, $\mathcal{R}_{1}-1>0$ and, therefore, $\lambda_{6}, \lambda_{7}$ and $\lambda_{8}$ in 6.12 are negative. However, $\lambda_{9}$ given in 6.12 is positive since $\overline{\beta_{2}}>\overline{\beta_{1}}$ and $\mathcal{R}_{1}-1>0$. Thus, $E_{1}$ is unstable.

For the analysis of the stability of the $E_{2}$, it is important to note that, since $E_{2}$ is in $\Omega, \mathcal{R}_{2}>1$ and, because of that, the eigenvalue in (6.14), $\lambda_{6}, \lambda_{7}$ and $\lambda_{8}$ are negative. Now, rewriting $\lambda_{9}$ which is given in 6.14 we obtain will be stable.

$$
\begin{aligned}
\lambda_{9} & =\frac{\overline{\alpha_{1}} \phi \gamma \omega_{2}\left(\mathcal{R}_{2}-1\right)}{\overline{\beta_{2}}\left(d+\omega_{2}\right)}+\frac{\overline{\beta_{1}}}{\overline{\beta_{2}}}(d+\gamma)-(d+\gamma) \\
& =\frac{\overline{\alpha_{1}} \phi \gamma \omega_{2}}{\overline{\beta_{2}}\left(d+\omega_{2}\right)}\left(\mathcal{R}_{2}-1\right)+\frac{\mathcal{R}_{1}}{\mathcal{R}_{2}}(d+\gamma)-(d+\gamma) .
\end{aligned}
$$

Thus, $\lambda_{9}<0$, if $\mathcal{R}_{s}=\frac{\mathcal{R}_{1}}{\mathcal{R}_{2}}+\left(\mathcal{R}_{2}-1\right) \frac{\overline{\alpha_{1}} \phi \gamma \omega_{2}}{\overline{\beta_{2}}\left(d+\omega_{2}\right)(d+\gamma)}<1$ and, the equilibrium $E_{2}$ 
be unstable.

And, $\lambda_{9}>0$, if $\mathcal{R}_{s}=\frac{\mathcal{R}_{1}}{\mathcal{R}_{2}}+\left(\mathcal{R}_{2}-1\right) \frac{\overline{\alpha_{1}} \phi \gamma \omega_{2}}{\overline{\beta_{2}}\left(d+\omega_{2}\right)(d+\gamma)}>1$ and, the equilibrium $E_{2}$ will In addition, if $\mathcal{R}_{s}=1$ then $S_{\max _{2}}=\frac{d+\gamma}{\overline{\beta_{2}}}$ will be the root of the polynomial $O(S)$, given only the boundary equilibrium (as described in the theorem for the existence of the equilibrium over the previous section in details).

We can rewrite the threshold value $\mathcal{R}_{s}$ defined in the theorem above as

$$
\begin{aligned}
\mathcal{R}_{\text {Inv }} & =\frac{\mathcal{R}_{1}}{\mathcal{R}_{2}}+\left(\mathcal{R}_{2}-1\right) \frac{\overline{\alpha_{1}} \phi \gamma \omega_{2}}{\overline{\beta_{2}}\left(d+\omega_{2}\right)(d+\gamma)} \\
& =\frac{\mathcal{R}_{1}}{\mathcal{R}_{2}}+\frac{\overline{\alpha_{1}} \phi}{\overline{\beta_{1}}} \frac{\gamma}{(d+\gamma)} \frac{\omega_{2}}{\left(d+\omega_{2}\right)} \mathcal{R}_{1}\left(1-\frac{1}{\mathcal{R}_{2}}\right) .
\end{aligned}
$$

$\mathcal{R}_{\text {Inv }}$ is also called the Invasion Reproduction number and we are going to discuss about it further, over the next chapters.

Note that, if $\frac{\overline{\alpha_{1}} \phi}{\overline{\beta_{1}}} \leq 1$, we have that $\mathcal{R}_{I n v}<\mathcal{R}_{1}$. Biologically speaking, these results mean that there is a range of values for $\beta_{1}$ for which the strain one cannot invade the population if the strain two is endemic. In this way, the strain two may protect the population from strain one. After this range value, the infections start to coexist.

The same result above from this theorem is valid for the case $\overline{\beta_{1}}>\overline{\beta_{2}}$.

\subsubsection{Symmetric Case}

On the symmetric case, the eigenvalues of Jacobian matrix at Disease Free equilibrium (DFE) of the system (1.8) are the same as in (6.10) taking the parameters equal. Then the eigenvalues are given by

$$
\begin{aligned}
& \lambda_{1}=-d \\
& \lambda_{2}=-(d+\omega) \\
& \lambda_{3}=-(d+\omega) \\
& \lambda_{4}=-(d+\gamma) \\
& \lambda_{5}=-(d+\gamma) \\
& \lambda_{6}=-d \\
& \lambda_{7}=-d \\
& \lambda_{8}=-(d+\gamma)+\beta N^{*} \\
& \lambda_{9}=-(d+\gamma)+\beta N^{*} .
\end{aligned}
$$


In the same way, taking the parameters equal in 6.12 or 6.14 , for the symmetric case, the eigenvalues of Jacobian matrix of the system (1.8) at

$$
E_{1}=\left(\frac{d+\gamma}{\beta}, \frac{d}{\beta}\left(\mathcal{R}_{0}-1\right), 0, \frac{\gamma}{d+\omega} I_{1}^{*}, 0, \frac{\omega}{d} \frac{\gamma}{d+\omega} I_{1}^{*}, 0,0,0\right)
$$

and, at

$$
E_{2}=\left(\frac{d+\gamma}{\beta}, 0, \frac{d}{\beta}\left(\mathcal{R}_{0}-1\right), 0, \frac{\gamma}{d+\omega} I_{2}^{*}, 0, \frac{\omega}{d} \frac{\gamma}{d+\omega} I_{2}^{*}, 0,0\right)
$$

are the same and they are given by

$$
\begin{aligned}
& \lambda_{1}=-d \\
& \lambda_{2}=-(d+\omega) \\
& \lambda_{3}=-(d+\omega) \\
& \lambda_{4}=-(d+\gamma) \\
& \lambda_{5}=-(d+\gamma) \\
& \lambda_{6}=-d\left(1+\frac{\alpha \phi}{\beta}\left(\mathcal{R}_{0}-1\right)\right) \\
& \lambda_{7}=\frac{1}{2}\left(-d \mathcal{R}_{0}-\sqrt{\left(d \mathcal{R}_{0}\right)^{2}-4 d(d+\gamma)\left(\mathcal{R}_{0}-1\right)}\right) \\
& \lambda_{8}=\frac{1}{2}\left(-d \mathcal{R}_{0}+\sqrt{\left(d \mathcal{R}_{0}\right)^{2}-4 d(d+\gamma)\left(\mathcal{R}_{0}-1\right)}\right) \\
& \lambda_{9}=\frac{\alpha \phi \gamma \omega\left(\mathcal{R}_{0}-1\right)}{\beta(d+\omega)} .
\end{aligned}
$$

This gives the following theorem about the stability of these equilibriums.

Theorem 15. If $\mathcal{R}_{0}<1$ then the Disease free equilibrium of the system $(1.8)$ is locally asymptotically stable on symmetric case. And it is unstable if $\mathcal{R}_{0}>1$. In addition, the Boundary equilibriums $E_{1}$ and $E_{2}$ given in (6.18) and (6.19), respectively, are unstable in the $\Omega$ region if $\mathcal{R}_{0}>1$.

Proof. If $\mathcal{R}_{0}<1$ then $N^{*} \beta<d+\gamma$. Therefore, the eigenvalues $\lambda_{8}$ and $\lambda_{9}$ in 6.17) are also negative. It proves the local stability of DFE. If $\mathcal{R}_{0}>1$ then $N^{*} \beta>d+\gamma$. And, the eigenvalues $\lambda_{8}$ and $\lambda_{9}$ in 6.17) are positive, consequently proving the instability of the DFE.

In addition, as we have seen in the previous theorem, $E_{1}$ and $E_{2}$ are in the region $\Omega$ if $\mathcal{R}_{0}>$ 1. Then, $N^{*} \beta>d+\gamma$ and the eigenvalue $\lambda_{6}$, given in 6.20), are negative. Also, $\lambda_{7}$ and $\lambda_{8}$ have negative real part, but $\lambda_{9}$ is positive, since $\mathcal{R}_{0}>1$. In that way, the Boundary equilibriums are always unstable. 
It is important to note that, when $\mathcal{R}_{0}=1$, we have $\frac{d+\gamma}{\beta}=N^{*}$. Therefore, the only equilibrium of the system is the Disease free equilibrium, in this case. Biologically speaking, these results mean that, since the serotypes have the same force of infection, if one strain invades a disease free population, also strain two, at same time. Thus, the two infection starts to coexist, at the same time, in a susceptible population.

\subsection{Numerical analysis: Particular case $\left(e^{-\omega_{i} t}\right)$}

\subsubsection{Stability of the Coexistence Endemic equilibrium}

As we have seen over the previous chapters, the local stability of the Coexistence Endemic equilibrium with the coexistence of the two serotypes has not been discussed yet. In this section, we are going to explore the analysis of this equilibrium numerically and, we are going to show the reason why we do not have successful results using the classical linearization theory.

\section{Symmetric case}

As we have seen over the chapter 2, concerning to the symmetric case, the theorem ensures a unique endemic equilibrium with the coexistence of two serotypes within the invariant region always exists when the Basic Reproduction Number is bigger than one. About this case in particular, the local stability of this endemic equilibrium has not been explored yet.

Although it was possible to describe analytically the equilibrium with coexistence of two strains, in the symmetric case, the expression for the value of $S^{*}$ shows a complex dependency of the parameters.

Thus concerning to the difficulty to work with a polynomial of nine degree, even with the help of the technical computing program Wolfram Mathematica, it is hard to describe the coefficients of the characteristic polynomial, as well as, its eigenvalues. Therefore, we are going to do a numerical analysis of the stability of the endemic equilibrium, analysing the sign of the real part of the eigenvalues of the matrix, numerically, for some values of the parameter $\phi$, using it as a bifurcation parameter.

Epidemiological speaking, $\phi$, the parameter used to describe the ADE effect, is unknown. Mathematically, once a value is given for $\phi$, we have a different value of $S^{*}$, consequently, for the eigenvalues of the Jacobian matrix. Thus, for all values of $\phi>0$, we already known that, there is a coexistence equilibrium and we can numerically verify its values and the correspondent eigenvalues, as we are going to show through the next figures. 


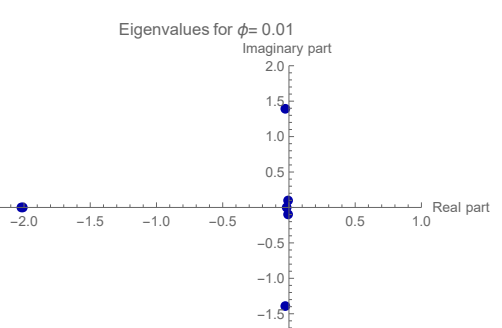

(a) $\phi=0.01$

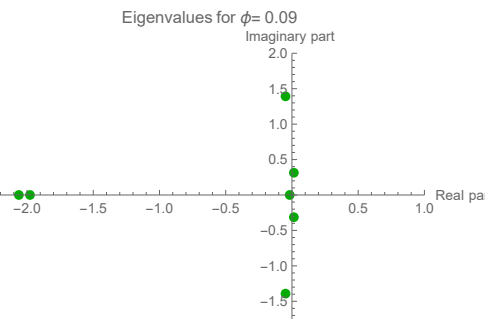

(d) $\phi=0.09$

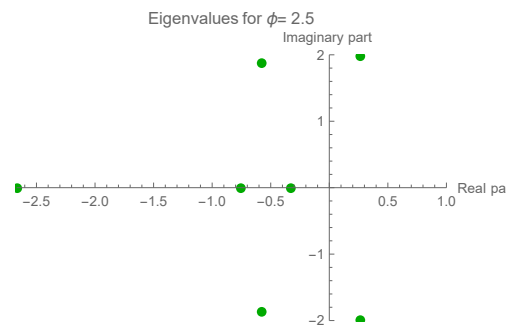

(g) $\phi=2.5$

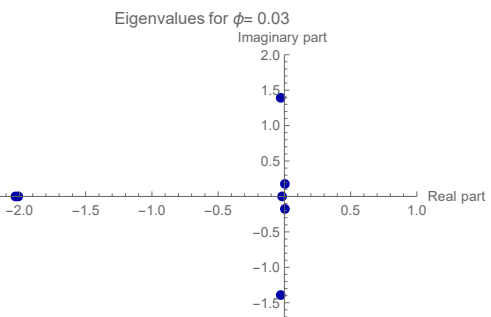

(b) $\phi=0.03$
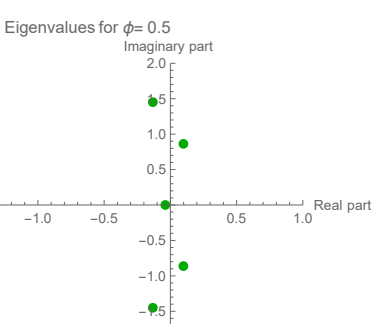

(e) $\phi=0.5$

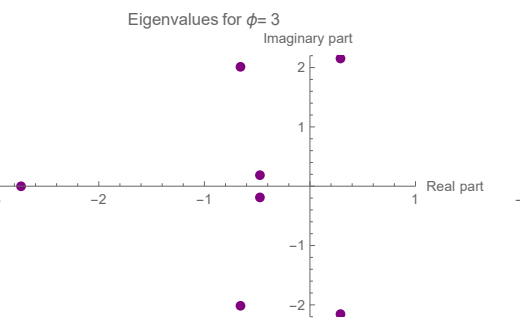

(h) $\phi=3$

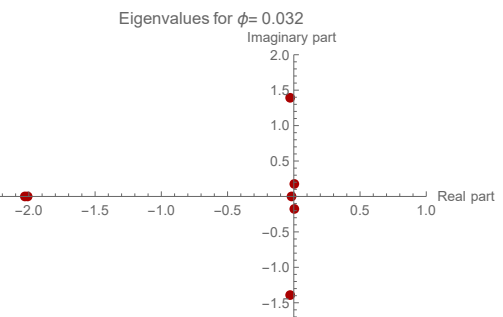

(c) $\phi=0.032$

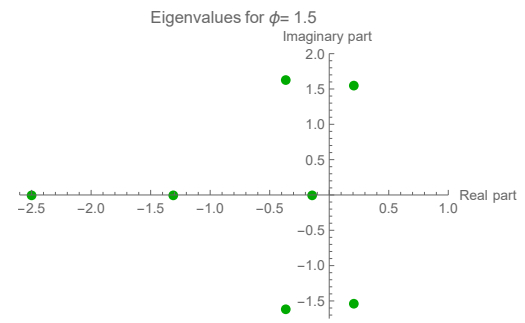

(f) $\phi=1.5$

Figure 6.1: Eigenvalues of the coexistence equilibrium in the complex plane, for each value of $\phi$. The values used in the simulations are found on table (3.2) with $\beta=\beta_{1}=\beta_{2}=180$ which gives $\mathcal{R}_{0}=3.46$. Figures $(6.1 \mathrm{c})$ shows that a purely imaginary eigenvalue appears at $\phi \approx 0.032$.

As it is known, a Hopf bifurcation arises when looking for the eigenvalues of the Jacobian matrix of the continuous parametric dynamical system evaluated at the equilibrium of it, all eigenvalues have negative real part except one conjugate non-zero purely imaginary pair that cross the imaginary axis, because of a variation of the parameter (Lynch, 2004, Martcheva, 2015).

Thus, analysing numerically the local stability of the endemic equilibrium $E_{3}$, figures 6.1 a $)$ to 6.1i) show that the matrix has nine equilibriums and five of them are negative, two of them are conjugated complex with negative real part and the other two are conjugated complex, changing the sign of the real part as $\phi$ increases. Thus, a Hopf bifurcation occurs when the parameters $\phi$ is $\approx 0.032$.

We can also see that when $\phi$ is near to 3 , the matrix has three negative real eigenvalues, one pair of complex conjugated with positive real part and two pairs of conjugated complex with negative 
real part. In this way, we have two negative real eigenvalues, changing to complex with negative real part eigenvalues, as shown on figures 6.1h and 6.1i).

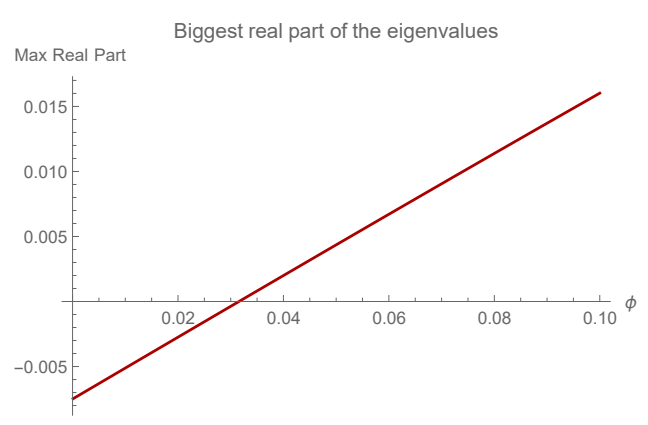

(a) $\phi \in(0,0.1)$

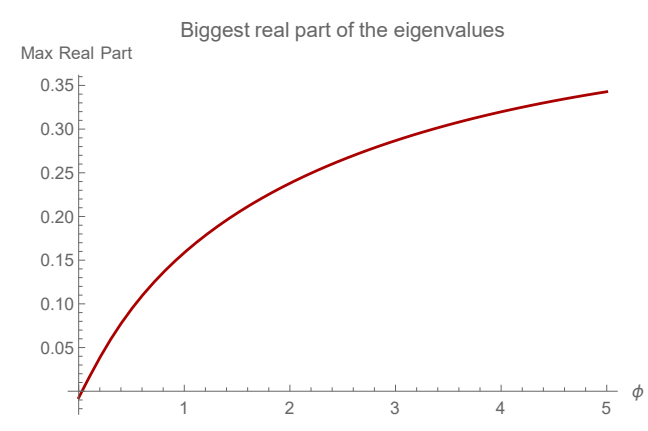

(b) $\phi \in(0,5)$

Figure 6.2: Maximum value of the real part of the eigenvalues of the endemic equilibrium. 6.2a shows a smaller range for $\phi$, highlighting the moment that the real part of the complex eigenvalues change its sign.

To complete this analysis, figures $6.2 \mathrm{a}$ and $6.2 \mathrm{~b}$ show the maximum of the real part of eigenvalues for each value of parameter $\phi$. Note the moment that $\phi$ approx the value 0.032 . The largest real part of the eigenvalues cross the $\mathrm{x}$-axis, when the Hopf bifurcation occurs and it remains positive.

\section{Asymmetric case}

As we have seen over the chapter 2 , the coexistence equilibrium $E_{3}$ exists only if the maximum of the Reproduction number is bigger than one, just in case the Invasion Reproduction number is bigger than one. In this case, counting with the existence of the two serotypes, the Boundary equilibrium loses stability and the coexistence equilibrium rises and remain in the invariant region.

It is possible to see on the figures $6.3 \mathrm{a}$ ) and $6.3 \mathrm{~b}$ ) the parameters region for the stability of the Boundary equilibriums, for the Disease Free equilibrium (DFE) and the parameters region for the existence of the Coexistence Endemic equilibrium. 


\section{Stability and existence region of the equilibriums}

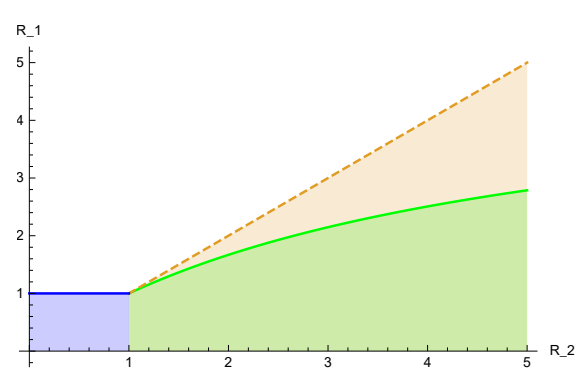

(a) $\phi=0.2$

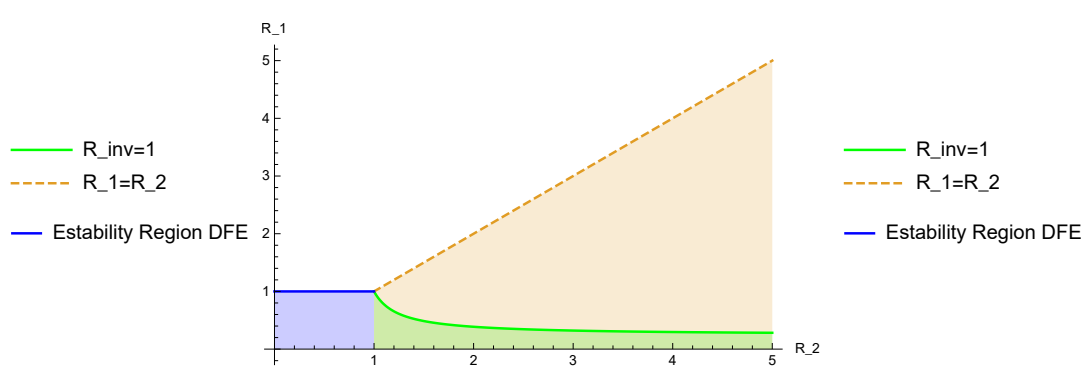

(b) $\phi=4.2$

Figure 6.3: The blue region represents the parameters region for which the DFE is globally stable $\left(\mathcal{R}_{0} \leq 1\right)$. The green region represents where the Boundary equilibrium is locally stable $\left(\mathcal{R}_{\text {Inv }} \leq 1\right)$. The coral one represents the existence region of the Coexistence Endemic equilibrium $\left(\mathcal{R}_{I n v}>1\right)$. Figure $6.3 \mathrm{a}$ with $\phi=0.2$ and figure $6.3 \mathrm{~b}$ with $\phi=4.2$.

Figures $6.3 \mathrm{a}$ and $6.3 \mathrm{~b}$ ) show that, as the value $\phi$ increases, the parameters region of the stability for the Boundary equilibrium decreases, forcing the endemic equilibrium to coexist within the region.

In the asymmetric case, it was not possible to describe analytically the equilibrium with coexistence of two strains in terms of the parameters because of the complex dependency on the parameters. However, we prove that it exists in theorem (11) of chapter 2.

Because of the difficulty to work with a polynomial of nine degree, even with the help of the technical computing program Wolfram Mathematica, it is hard to describe the coefficients of the characteristic polynomial, as well as, the eigenvalues. As a consequence, we are going to do a numerical analysis of the stability of the endemic equilibrium, analysing the sign of the real part of the eigenvalues of the matrix, numerically, for some values of the parameter $\phi$.

Epidemiological speaking, $\phi$, the parameter used to describe the ADE effect is a parameter that its value is unknown. Mathematically, once it is given a value for $\phi$ we have a different value of $S^{*}$ and, therefore for the eigenvalues of the Jacobian matrix.

As we have seen on the figures $6.3 \mathrm{a}$ and $6.3 \mathrm{~b}$ there is a threshold for the value of $\phi$, in each case, which satisfies $\mathcal{R}_{I n v}>1$. Then, starting for this threshold value, the Coexistence equilibrium will be in the positive region and, therefore, we can look for the eigenvalues. Thus, we can numerically verify the local dynamic near the correspondent endemic equilibrium for each value of $\phi$.

In the asymmetric case, we choose two cases:

Case (i): $\mathcal{R}_{0}>1, \mathcal{R}_{1}<1$

In this case, with values for the parameters on table 3.2 with $\beta_{1}=45$ and $\beta_{2}=180$, which give $\mathcal{R}_{1}=0.87$, and $\mathcal{R}_{0}=3.46$, the $\mathcal{R}_{\text {Inv }}$ is bigger than one for $\phi$ bigger than 1.23 . Then, for all values 
of $\phi>1.23$ we have the existence of the coexistence endemic equilibrium and the correspondents eigenvalues as shown on the figures.

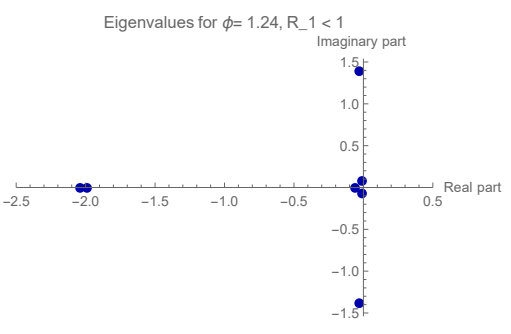

(a) $\phi=1.24$

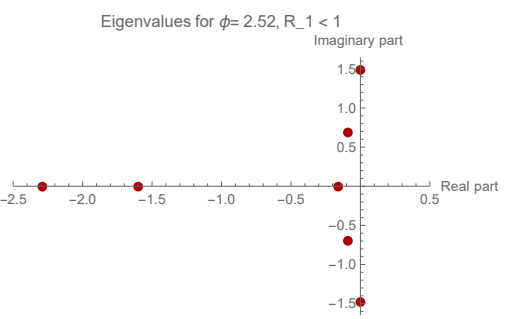

(d) $\phi=2.52$

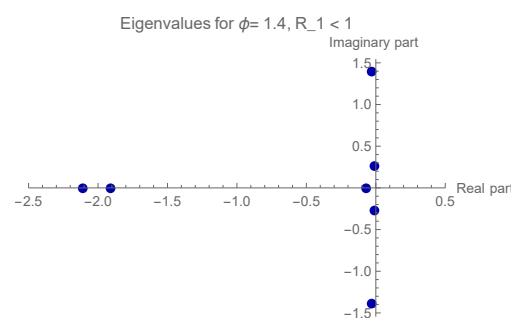

(b) $\phi=1.4$
Eigenvalues for $\phi=2.8, \mathrm{R}_{-} 1<1$

(e) $\phi=2.8$

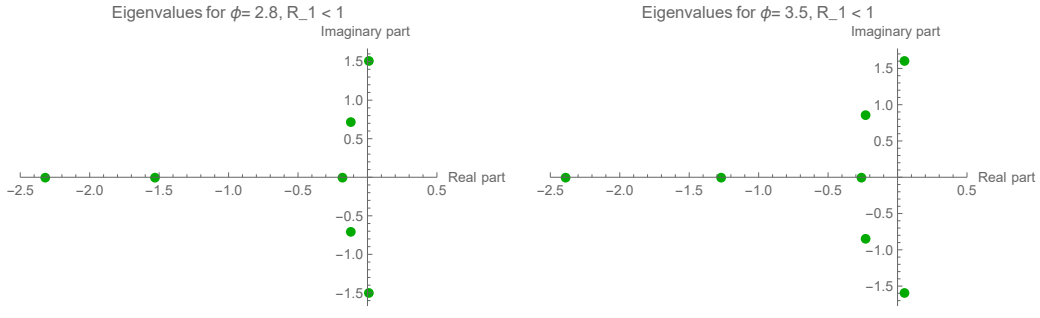

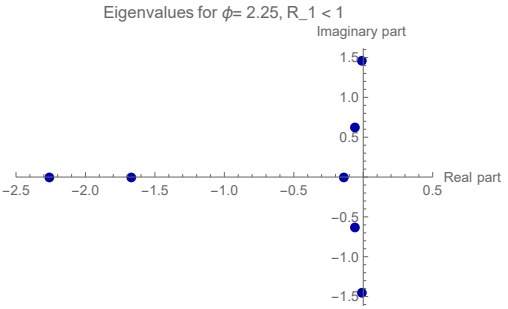

(c) $\phi=2.25$

Figure 6.4: Eigenvalues of the coexistence endemic equilibrium in the complex plane, for each value of $\phi$. Figures 6.4a to the show that the real part of a pair of complex eigenvalues change the sign as $\phi$ increases.

Analysing numerically the local stability of the coexistence endemic equilibrium $E_{3}$, we can see that the matrix has nine equilibriums of which five are negative real, two are conjugated complex with negative real part, and the other two conjugated complex change the sign of the real part as $\phi$ increases. Thus, a Hopf bifurcation occurs when the parameters $\phi$ is $\approx 2.52$ (figure $6.4 \mathrm{~d}$ ).

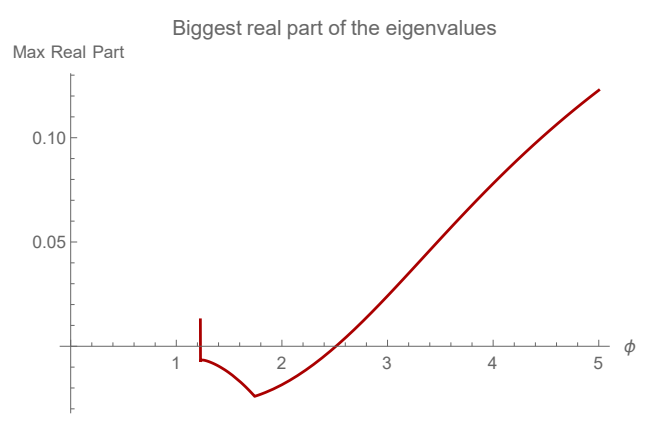

(a) $\phi \in(1.2,5)$

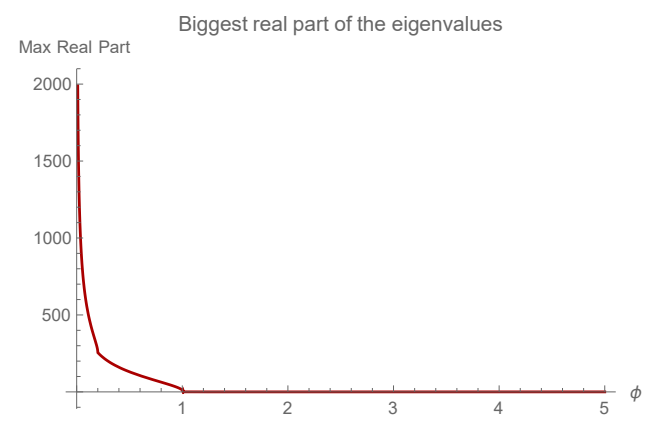

(b) $\phi \in(0,5)$

Figure 6.5: Maximum value of the real part of the eigenvalues of the endemic equilibrium. 6.5a) shows a smaller range for $\phi$, highlighting the moment that the real part of the complex eigenvalues change its sign. 
To complete the analysis on the figures $6.5 \mathrm{a})$ and $(6.5 \mathrm{~b})$, it is possible to note the moment that the biggest real part of the eigenvalues cross the $\mathrm{x}$-axis and remain positive, close to $\phi=2.52$, when the Hopf Bifurcation occurs.

It is also possible to see that as $\phi$ approx the value 1.23 , the real part of the eigenvalue change the sign. It is the exact moment that the Invasion Reproduction number is bigger than one, and therefore, the equilibrium is positive, in the positive invariant region and, it inherits the stability of the boundary equilibrium, having as a consequence negative eigenvalues for $\phi \in(1.23,2.52)$.

Case (ii): $\mathcal{R}_{0}>1, \mathcal{R}_{1}>1$

In this case, with the values for the parameters on the table (3.2) with $\beta_{1}=120$ and $\beta_{2}=180$, which give $\mathcal{R}_{1}=2.31$, and $\mathcal{R}_{0}=3.46$, the $\mathcal{R}_{\text {Inv }}$ is bigger than one for $\phi$ bigger than 0.21 . Then, for all values of $\phi>0.21$ we have the existence of the coexistence endemic equilibrium and the correspondents eigenvalues, as shown on the figures.

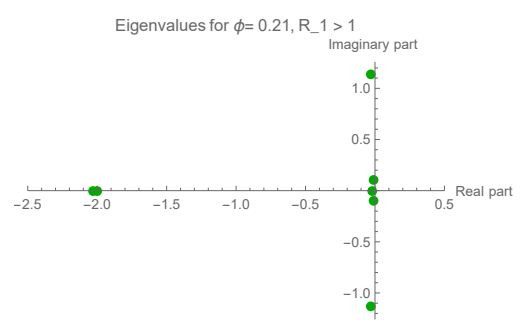

(a) $\phi=0.21$

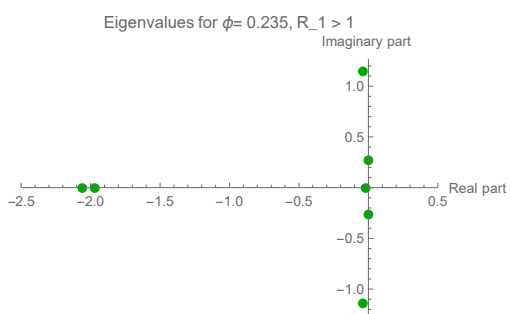

(b) $\phi=0.235$

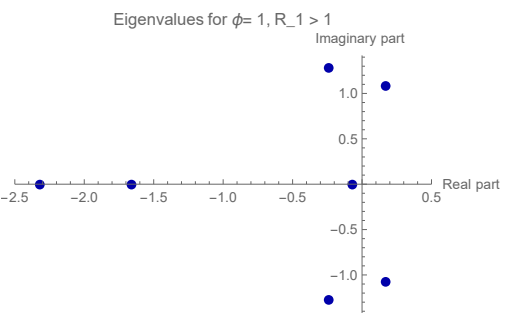

(e) $\phi=1$

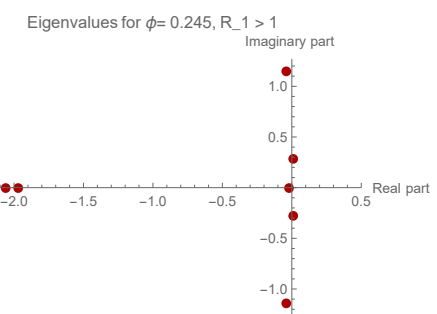

(c) $\phi=0.244$

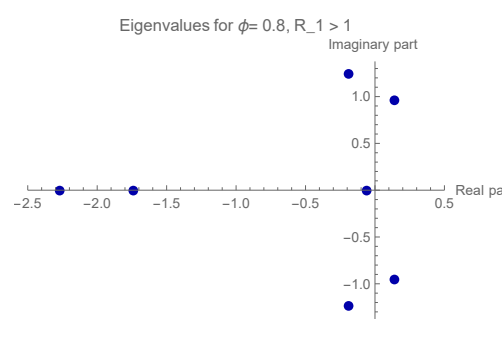

(d) $\phi=0.8$

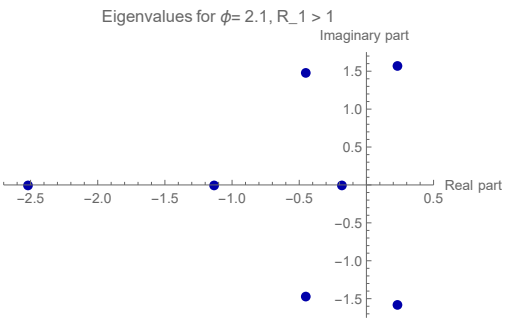

(f) $\phi=2.1$

Figure 6.6: Eigenvalues of the coexistence endemic equilibrium in the complex plane, for each value of $\phi$. Figures $6.6 \mathrm{a}$ to th.6f show that the real part of a pair of complex eigenvalues changes the sign as $\phi$ increases.

Analysing numerically the local stability of the endemic equilibrium $E_{3}$, we can see that the matrix has nine equilibriums of which five are negative, two are conjugated complex with negative real part and the other two conjugated complex change the sign of the real part as $\phi$ increases. Thus, a Hopf bifurcation occurs when parameters $\phi \approx 0.244$ (figure $6.6 \mathrm{c}$ ). 


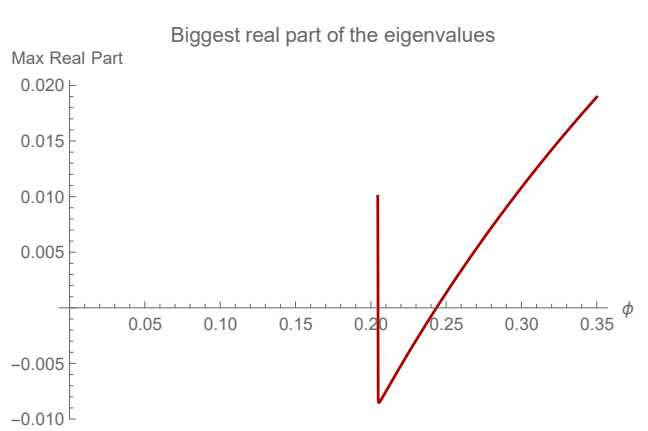

(a) $\phi \in(0.2,0.35)$

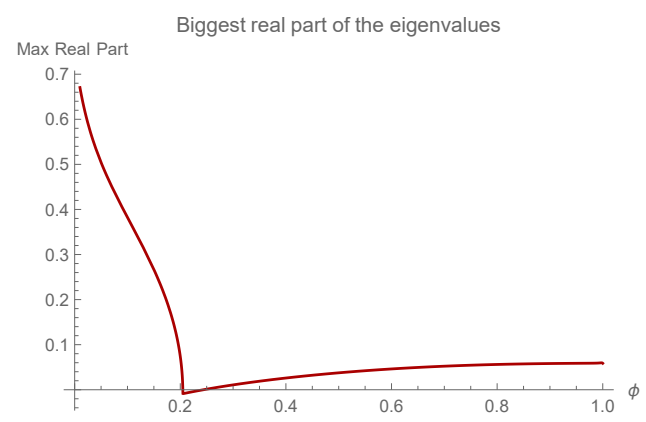

(b) $\phi \in(0,1)$

Figure 6.7: Maximum value of the real part of the eigenvalues of the endemic equilibrium. 6.7a shows a smaller range for $\phi$, highlighting the moment that the real part of the complex eigenvalues changes its sign.

To complete the analysis on the figures $6.5 \mathrm{a}$ and $6.5 \mathrm{~b}$, it is possible to note the moment that the biggest real part of the eigenvalues crosses the $\mathrm{x}$-axis and remains positive, close to $\phi=0.244$, when the Hopf Bifurcation occurs.

It is also possible to see that as $\phi$ approx the value 0.21 , the real part of the eigenvalue changes the sign. It is the exact moment that the Invasion Reproduction number is bigger than one, the equilibrium is positive in the positive invariant region and it inherits the stability of the boundary equilibrium, having as consequence, negative eigenvalues for $\phi \in(0.21,0.244)$.

\section{Bifurcation Structure}

As we have showed numerically on the pictures in the previous sections that the Endemic coexistence equilibrium changes the stability as the parameter $\phi$ changes. As $\phi$ increases from small values through critical value, $\phi_{c}$, the steady state changes from a stable focus to an unstable steady state. Therefore, Hopf bifurcation occurs and we are able to conclude that closed periodic orbit will be found in a small neighbourhood of $\phi_{c}$.

According to Lynch (2004), two types of Hopf bifurcation can occur: the supercritical Hopf bifurcation, in which stable limit cycle is created surrounding an unstable critical point; and the subcritical Hopf bifurcation, in which an unstable limit cycle is created and it surrounds a stable critical point.

In order to see the limit cycle around the equilibrium, in a small vicinity of the critical value, bifurcation diagrams are shown on the next figures. 


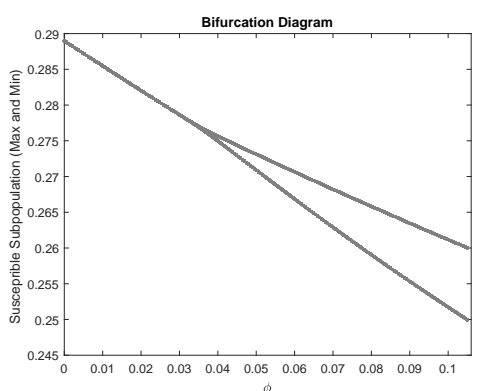

(a) Symmetric case ( $\left.\phi_{c}=0.032\right)$

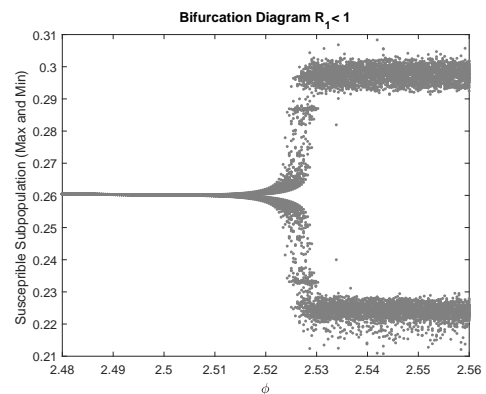

(b) Asymmetric case: $\mathcal{R}_{1}=0.87\left(\phi_{c}=2.52\right)$

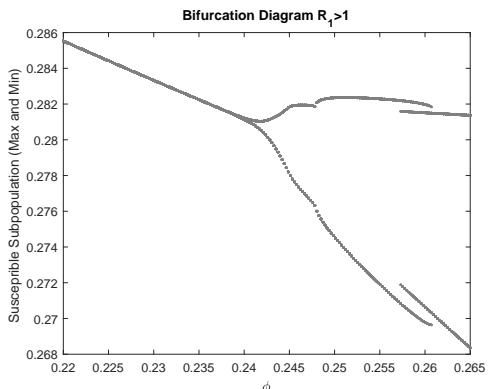

(c) Asymmetric case: $\mathcal{R}_{1}=2.31\left(\phi_{c}=0.244\right)$

Figure 6.8: Bifurcation diagrams for symmetric and asymmetric cases. In the horizontal axis, the parameter $\phi$ varies in a vicinity of $\phi_{c}$, while in the vertical axis, the maximum and minimum values for susceptible population are plotted.

At symmetric case, the Hopf bifurcation occurs at $\phi_{c}=0.032$, as a consequence, the solutions exhibit a small amplitude limit cycle around the endemic equilibrium. A stable limit cycle clearly arises and goes away from the equilibrium (figure (6.8a)). Also, the amplitude of the limit cycle increases gradually as the parameter $\phi$ moves away from the critical bifurcation point. Thus, it is possible to conclude that a supercritical Hopf bifurcation occurred.

At asymmetric case, the Hopf bifurcation occurs at $\phi_{c}=2.52$ and $\phi_{c}=0.244$, as a consequence, the solutions exhibit a small amplitude limit cycle around the endemic equilibrium. A stable limit cycle arises close to the critical Bifurcation point and goes away from the unstable equilibrium (figure (6.8b) ) and (figure (6.8c). Thus, it is possible to conclude that a supercritical Hopf bifurcation occurred.

This change of stability, in other words, this kind of bifurcation is local. Therefore, the Hopf bifurcation does not specify what happens when the parameter is further beyond the vicinity of its critical bifurcation value (Edelstein-Keshet, 2005; Murray, 2002).

Because of that, solutions will be plotted in the next section for different values of $\phi$, in order to support the theoretical analysis (about stability of the equilibriums), verify the local numerical analysis (about the H. B.), as well as showing the asymptotic behaviour for parameter values further from bifurcation value.

\subsubsection{Solutions of the system}

In this section, we are going to explore and to analyse numerically the solutions of the system in order to understand better the model, as well as using this numerical approach to obtain information about the solutions of the system for values of parameter $\phi$ further from the bifurcation value. 


\section{Symmetric case}

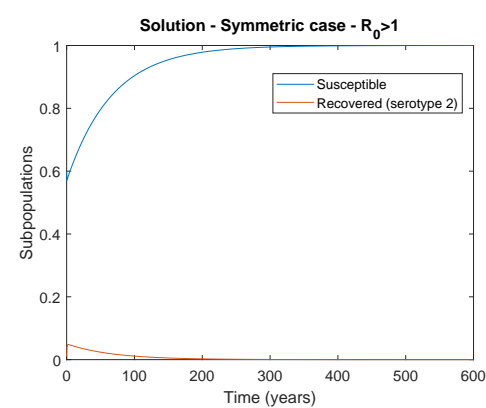

(a) $\phi=1$

Figure 6.9: Solutions of the system for $\phi=1, \beta=45, \mathcal{R}_{0}=0.87<1$.Initial conditions: $(0.6,0.01,0.02,0,0,0,0.01,0,0)$.

The figure 6.9a shows the solutions of the system at the symmetric case, for the parameter values on the table (3.2) with $\phi=1, \beta=45$ giving $\mathcal{R}_{0}=0.87<1$. In this case, the long term behaviour of the solutions tends to the DFE.

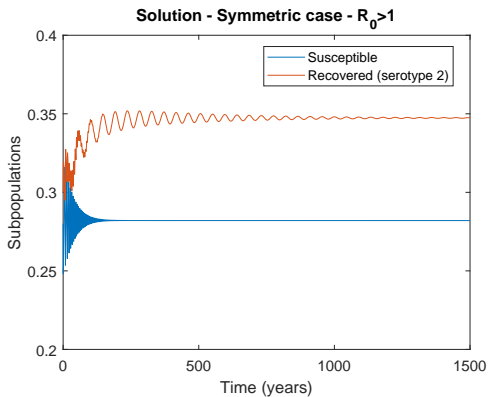

(a) $\phi=0.02$

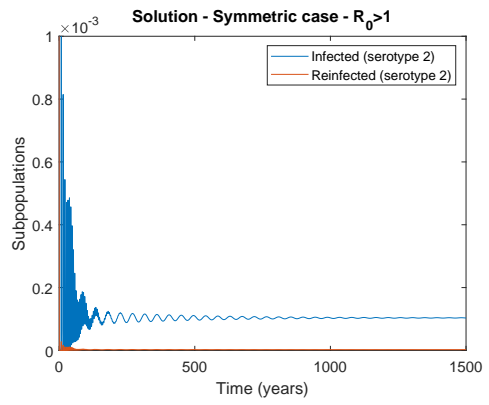

(b) $\phi=0.02$

Figure 6.10: Solutions of the system for $\phi=0.02, \beta=180, \mathcal{R}_{0}=3.46>1$ Initial condition $(0.28,0.00011,0.0011,0.002,0.002,0.3,0.3,0.001,0.001)$.

The figures 6.10a and 6.10b show the solutions of the system at the symmetric case, for the parameter values on the table 3.2 with $\phi=0.02, \beta=180$ giving $\mathcal{R}_{0}=3.46>1$. In this case, the long term behaviour of the solutions tends to the coexistence (endemic) equilibrium. This happens for all values of $\phi \in(0,0.032)$. 


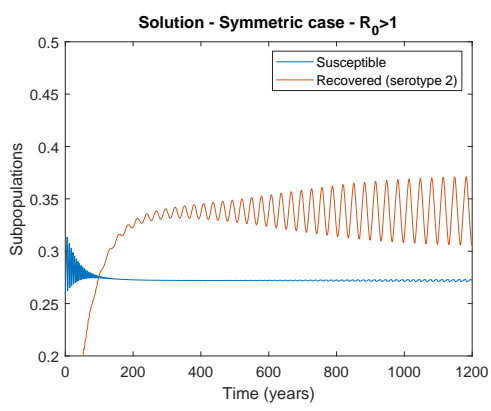

(a) $\phi=0.05$

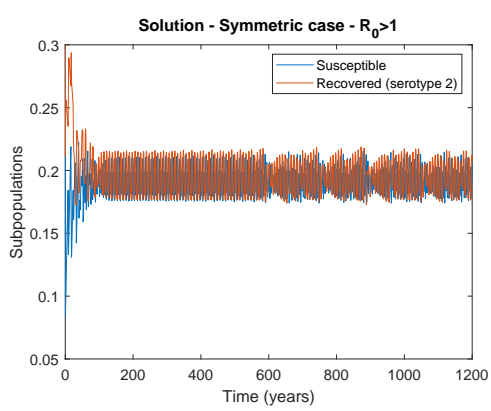

(d) $\phi=0.5$

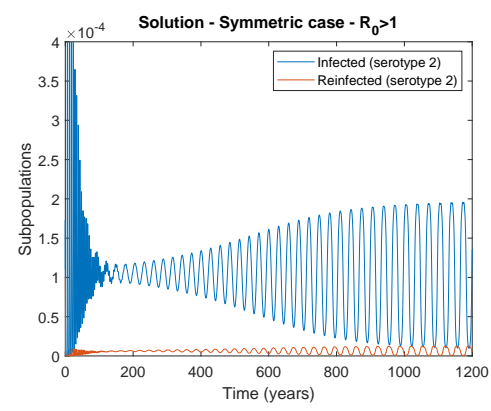

(b) $\phi=0.05$

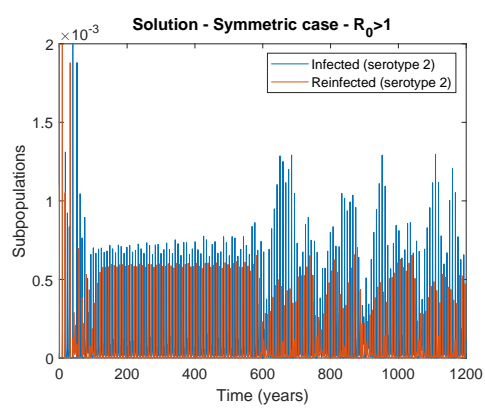

(e) $\phi=0.5$

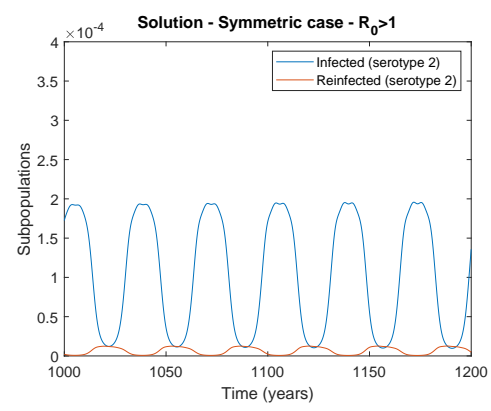

(c) $\phi=0.05$

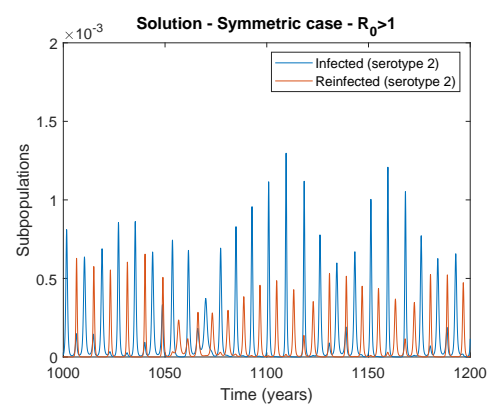

(f) $\phi=0.5$

Figure 6.11: Solutions of the system for $\beta=180, \mathcal{R}_{0}=3.46>1$. For $\phi=0.05$, initial condition $(0.26,0.00011,0.00015,0.003,0.004,0.034,0.036,0.00010,0.00012)$. For $\phi=0.5$, initial condition $(0.3,0.0011,0.002,0.01,0.02,0.2,0.3,0.001,0.002)$.

The figures 6.11a to 6.11c show the solutions of the system at the symmetric case, parameter values on the table 3.2 with $\phi=0.05, \beta=180$ giving $\mathcal{R}_{0}=3.46>1$. And figures 6.11d to 6.11f, for $\phi=0.5$. In the first case, the equilibrium is unstable and the value for $\phi$ is taken close to the Hopf Bifurcation value and, as a consequence, closed periodic orbits will be found. The solutions indicate that the limit cycle appears and it is stable for small values of $\phi$ close to $\phi_{c}=0.032$.

As $\phi$ increases far from the bifurcation critical value, a change in the behaviour of the solutions is seen ( figures 6.11d and 6.11e). In this case, the solutions of the system for $\phi=0.5$ seem to converge at the beginning to a periodic orbit. However, for long term behaviour, it is possible to see different oscillations and a complex behaviour $6.11 \mathrm{f})$. 


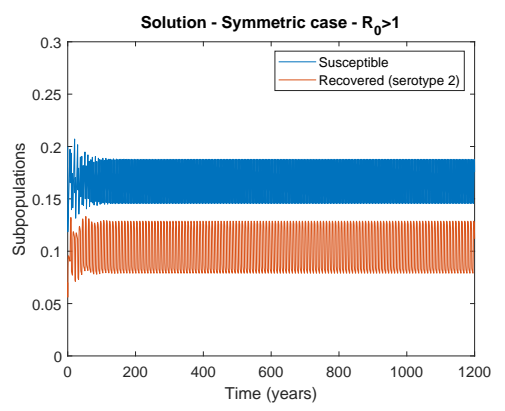

(a) $\phi=1.2$

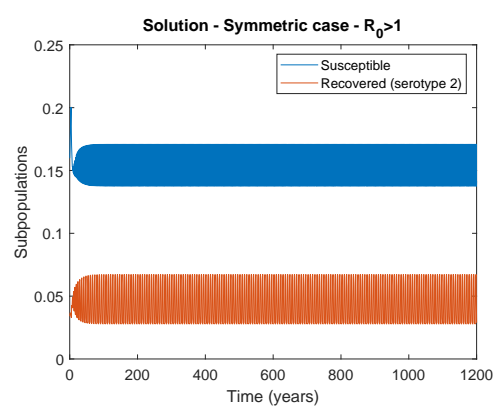

(d) $\phi=3$

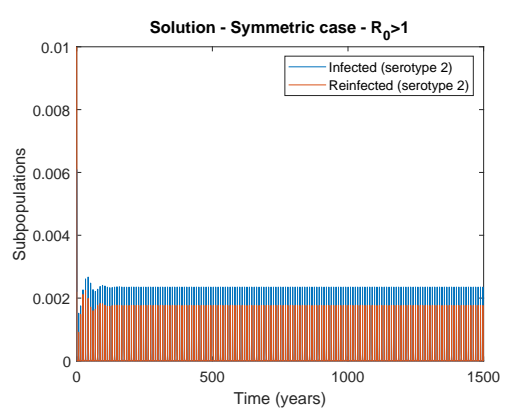

(b) $\phi=1.2$

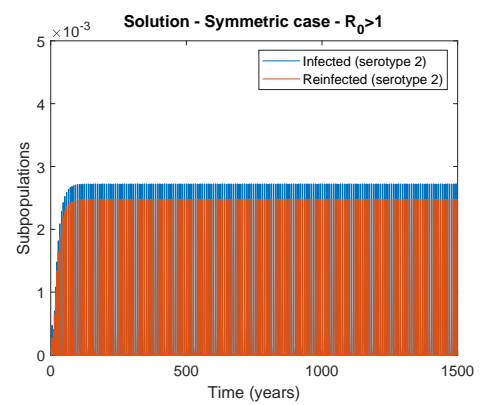

(e) $\phi=3$

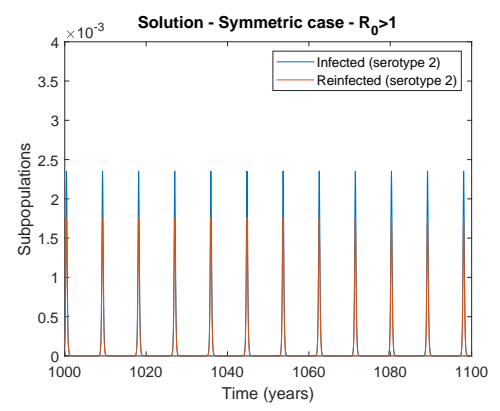

(c) $\phi=1.2$

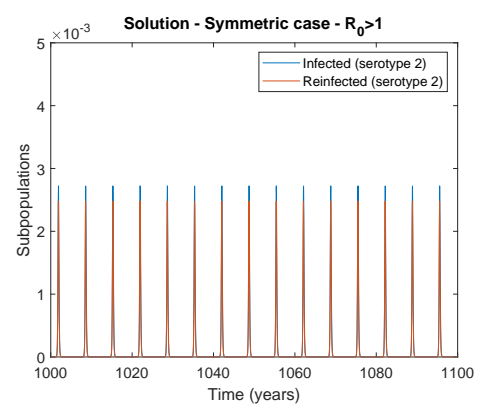

(f) $\phi=3$

Figure 6.12: Solutions of the system for $\phi=3, \beta=180$. For $\phi=1.2$, initial condition $(0.2,0.0011,0.0022,0.01,0.02,0.07,0.07,0.01,0.02)$. For $\phi=3$, initial condition $(0.16,0.00011,0.00013,0.003,0.003,0.034,0.034,0.00012,0.0001)$.

The figures 6.12a to 6.12f show the solutions of the system at the symmetric case for the parameter values on the table 3.2 with $\beta=180$ giving $\mathcal{R}_{0}=3.46>1$. In both cases, $\phi=1.2$ and $\phi=3$, the solutions of the system converge to periodic orbits.

\section{Asymmetric case}

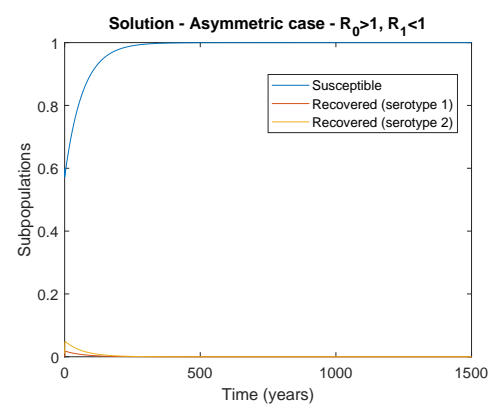

(a) $\phi=1$

Figure 6.13: Solutions of the system for $\phi=1, \beta_{1}=40, \mathcal{R}_{1}=0.76<1, \beta_{2}=45, \mathcal{R}_{2}=$ $0.87<1$. Initial conditions: $(0.6,0.01,0.02,0,0,0,0.01,0,0)$. 
The figure 6.13a shows the solutions of the system at the asymmetric case for the parameter values on the table (3.2) with $\phi=1, \beta_{1}=40$ giving $\mathcal{R}_{1}=0.76<1$, and $\beta_{2}=45$ giving $\mathcal{R}_{0}=0.87<$ 1. In this case, the long term behaviour of the solutions tends to the DFE.

Now, we are going to explore and to analyse numerically the solutions of the system in the asymmetric case, when $\beta_{1}<\beta_{2}$. We are going to divided in two cases: case (i), when $\mathcal{R}_{1}<1<\mathcal{R}_{2}$ and case (ii), when $1<\mathcal{R}_{1}<\mathcal{R}_{2}$.

Case (i): $\mathcal{R}_{0}>1, \mathcal{R}_{1}<1$

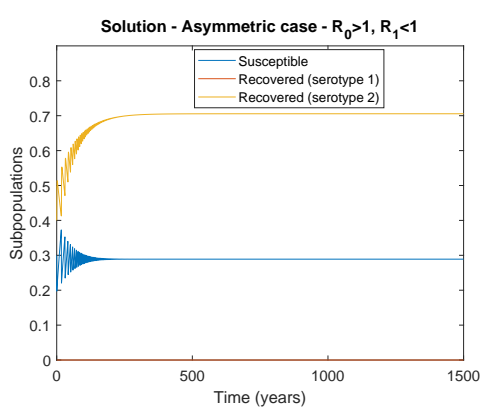

(a) $\phi=0.5$

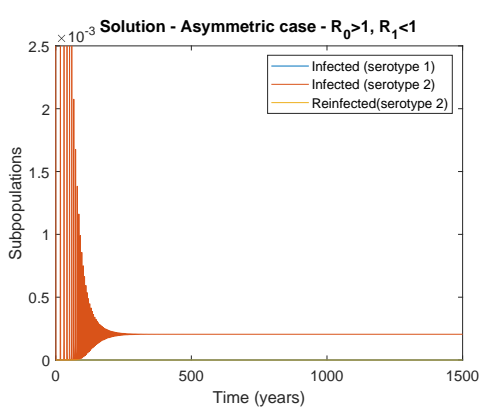

(b) $\phi=0.5$

Figure 6.14: Solutions of the system for $\phi=0.5, \beta_{1}=45, \mathcal{R}_{1}=0.87<1, \beta_{2}=180$, $\mathcal{R}_{2}=3.46>1$. Initial conditions: $(0.3,0.000001,0.02,0,0.005,0,0.4,0,0)$.

The figures (6.14a) and (6.14b) show the solutions of the system at the asymmetric case for the parameter values on the table 3.2 with $\phi=0.5, \beta_{1}=45$ giving $\mathcal{R}_{1}=0.87<1$ and, $\beta_{2}=180$ giving $\mathcal{R}_{2}=3.46>1$. In this case, the long term behaviour of the solutions tends to the Boundary equilibrium $E_{2}$, in other words, the Boundary equilibrium is locally asymptotically stable. In addition, the coexistence equilibrium is not in the positive region. This happens for all values of $\phi \in(0,1.23)$. 


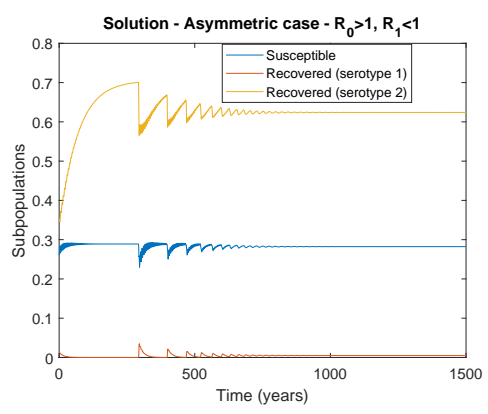

(a) $\phi=1.4$

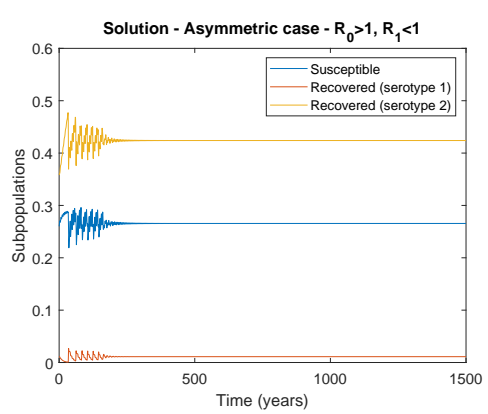

(c) $\phi=2.1$

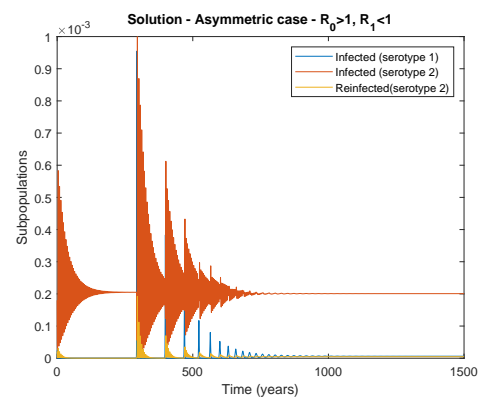

(b) $\phi=1.4$

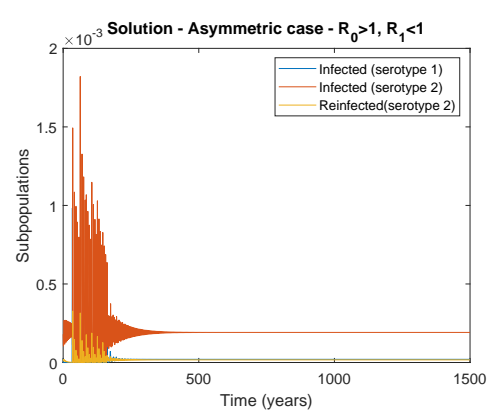

(d) $\phi=2.1$

Figure 6.15: Solutions of the system for $\phi=1.4, \beta_{1}=45, \mathcal{R}_{1}=0.87<$ $1, \beta_{2}=180, \mathcal{R}_{2}=3.46>1$. For $\phi=1.4$, initial conditions $(0.26,0.000024,0.00018,0.00063,0.0048,0.012,0.35,0.000021,0.000085) . \quad$ For $\phi=2.1$, initial conditions $(0.2601,0.0000244,0.00018,0.00063,0.0048,0.0115,0.358,0.0000209,0.000084)$.

The figures 6.15a and 6.15b) show the solutions of the system at the asymmetric case for the parameter values on the table (3.2) for $\phi=1.4, \beta_{1}=45$ giving $\mathcal{R}_{1}=0.87<1$ and, $\beta_{2}=180$ giving $\mathcal{R}_{2}=3.46>1$. And, for $\phi=2.1$, the solutions are shown by figures $6.15 \mathrm{c}$ ) and $6.15 \mathrm{~d}$. In both cases, the long term behaviour of the solutions converges to the Coexistence equilibrium, it means that the Coexistence equilibrium is locally asymptotically stable. This happens for all values of $\phi \in(1.23,2.52)$. 


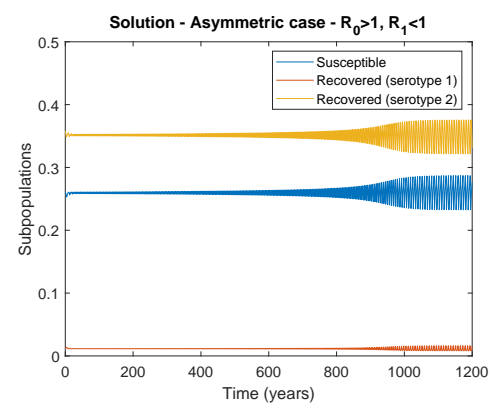

(a) $\phi=2.55$

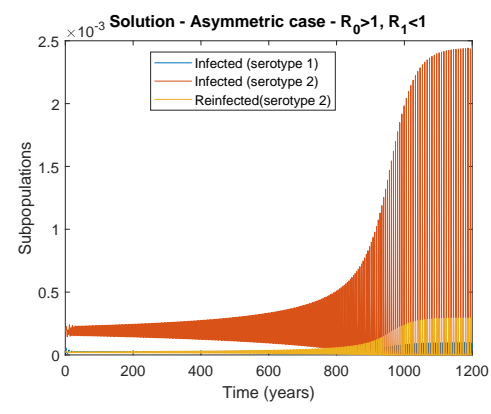

(b) $\phi=2.55$

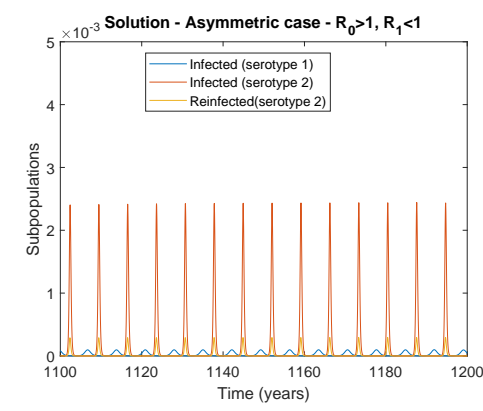

(c) $\phi=2.55$

Figure 6.16: Solutions of the system for $\phi=2.55, \quad \beta_{1}=45$, $\mathcal{R}_{1}=0.87<1, \beta_{2}=180, \mathcal{R}_{2}=3.46>1 . \quad$ Initial conditions: $(0.2601,0.0000244,0.00018,0.00063,0.0048,0.0115,0.358,0.000020,0.00008)$.

The figures (6.16a) to (6.16c) show the solutions of the system at the asymmetric case for the parameter values on the table (3.2) for $\phi=2.55, \beta_{1}=45$ giving $\mathcal{R}_{1}=0.87<1$ and, $\beta_{2}=180$ giving $\mathcal{R}_{2}=3.46>1$. In this case, the parameter $\phi$ is taken close to the value of Hopf Bifurcation at $\phi_{c}=2.52$ and the solution exhibit for a long time behaviour a limit cycle.

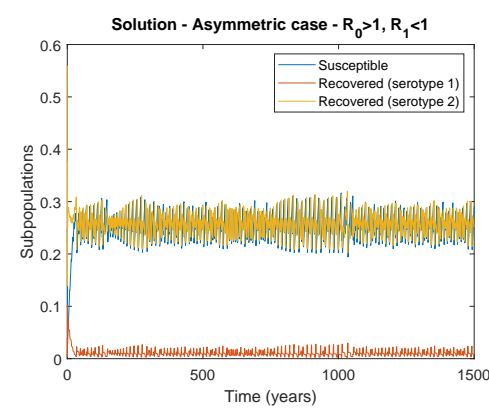

(a) $\phi=3.5$

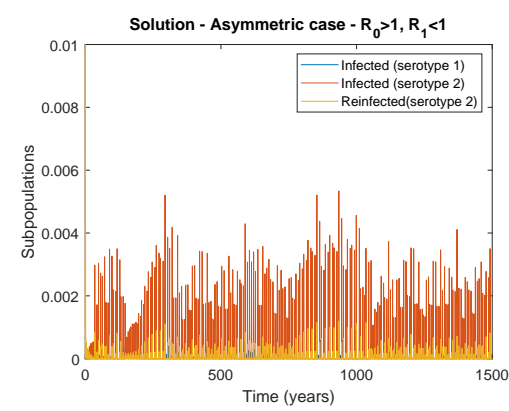

(b) $\phi=3.5$

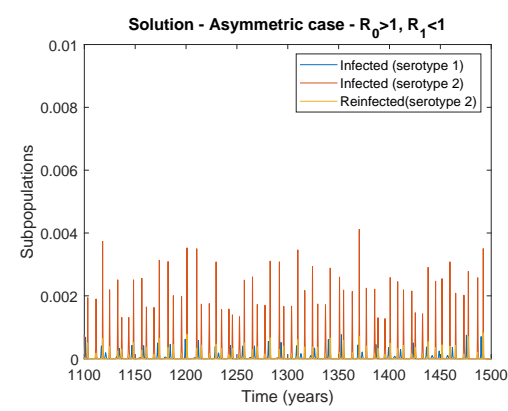

(c) $\phi=3.5$

Figure 6.17: Solutions of the system for $\phi=3.5, \beta_{1}=45, \mathcal{R}_{1}=0.87<1, \beta_{2}=180$, $\mathcal{R}_{2}=3.46>1$. Initial conditions: $(0.25,0.08,0.2,0.05,0.15,0.04,0.56,0.001,0.009)$.

The figures 6.17a to 6.17c show the solutions of the system at the asymmetric case for the parameter values on the table (3.2) with $\phi=3.5, \beta_{1}=45$ giving $\mathcal{R}_{1}=0.87<1$ and, $\beta_{2}=180$ giving $\mathcal{R}_{2}=3.46>1$. In this case, the solutions show a complex behaviour.

Now, we are going to explore and to analyse numerically the solutions of the system in the asymmetric case, when $1<\mathcal{R}_{1}<\mathcal{R}_{2}$.

Case (ii): $\mathcal{R}_{0}>1, \mathcal{R}_{1}>1$ 


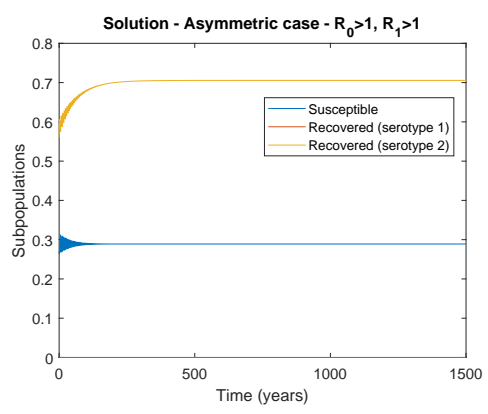

(a) $\phi=0.1$

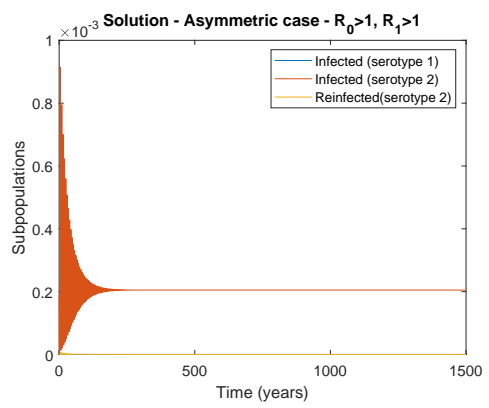

(b) $\phi=0.1$

Figure 6.18: Solutions of the system for $\phi=0.1, \beta_{1}=120, \mathcal{R}_{1}=2.31>1, \beta_{2}=180$, $\mathcal{R}_{2}=3.46>1$. Initial conditions: $(0.27,0.000001,0.001,0.00001,0.05,0,0.56,0,0)$.

The figures (6.18a) and (6.18b) show the solutions of the system at the asymmetric case for the parameter values on the table (3.2) with $\phi=0.1, \beta_{1}=120$ giving $\mathcal{R}_{1}=2.31>1$ and, $\beta_{2}=180$ giving $\mathcal{R}_{2}=3.46>1$. In this case, the long term behaviour of the solutions tends to the Boundary equilibrium $E_{2}$, it means that the Boundary equilibrium is locally asymptotically stable. In addition, the Coexistence equilibrium is not in the positive region. This happens for all values of $\phi \in(0,0.21)$.

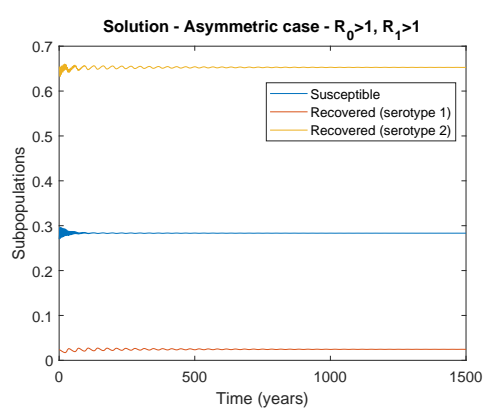

(a) $\phi=0.23$

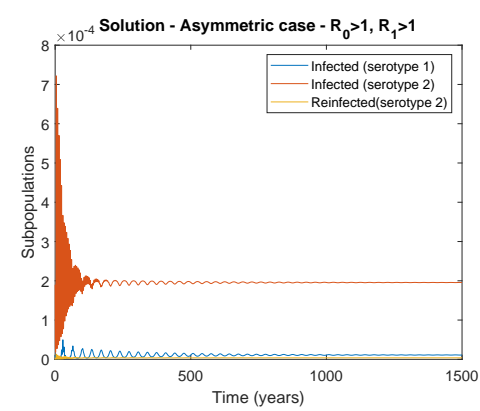

(b) $\phi=0.23$

Figure 6.19: Solutions of the system for $\phi=0.23, \quad \beta_{1}=120$, $\mathcal{R}_{1}=2.31>1, \beta_{2}=180, \mathcal{R}_{2}=3.46>1 . \quad$ Initial conditions:(0.27, 0.000011, 0.00019, 0.00027, 0.00505, 0.0237, 0.6546, 0.0000031, 0.0000011).

The figures (6.19a) and (6.19b) show the solutions of the system at the asymmetric case for the parameter values on the table 3.2 with $\phi=0.23, \beta_{1}=120$ giving $\mathcal{R}_{1}=2.31>1$ and, $\beta_{2}=180$ giving $\mathcal{R}_{2}=3.46>1$. In this case, the long term behaviour of the solutions tends to the Coexistence equilibrium, it means that the Coexistence equilibrium is locally asymptotically stable. This happens for all values of $\phi \in(0.21,0.244)$. 


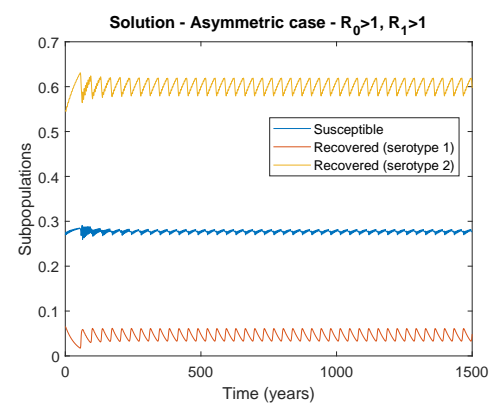

(a) $\phi=0.26$

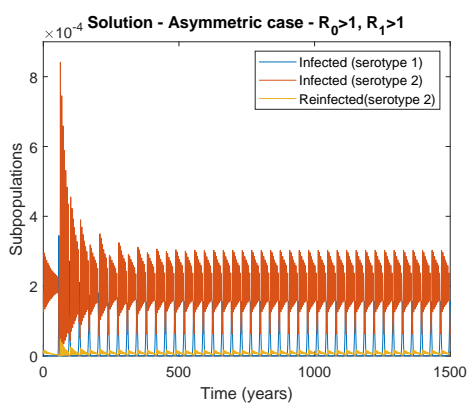

(b) $\phi=0.26$

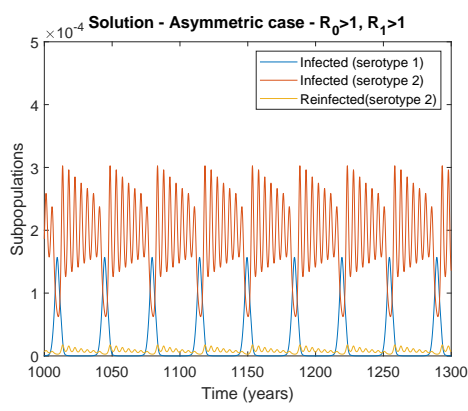

(c) $\phi=0.26$

Figure 6.20: Solutions of the system for $\phi=0.26, \quad \beta_{1}=120$, $\mathcal{R}_{1}=2.31>1, \beta_{2}=180, \mathcal{R}_{2}=3.46>1 . \quad$ Initial conditions: $(0.269,0.0000321,0.0001786,0.000828,0.00461,0.0653,0.546,0.0000129,0.0000195)$.

The figures 6.20a to 6.20c show the solutions of the system at the asymmetric case for the parameter values on the table (3.2) with $\phi=0.26, \beta_{1}=120$ giving $\mathcal{R}_{1}=2.31>1$ and, $\beta_{2}=180$ giving $\mathcal{R}_{2}=3.46>1$. In this case, a stable limit cycle appears and the solutions converge to a periodic orbit.

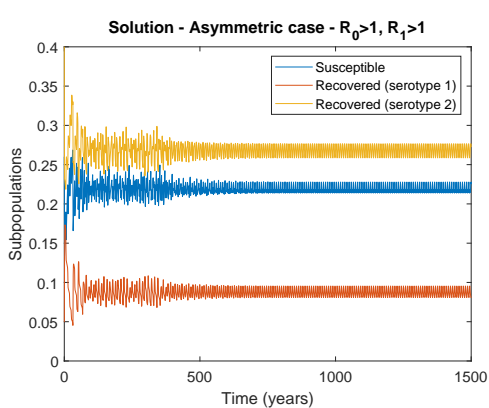

(a) $\phi=0.8$

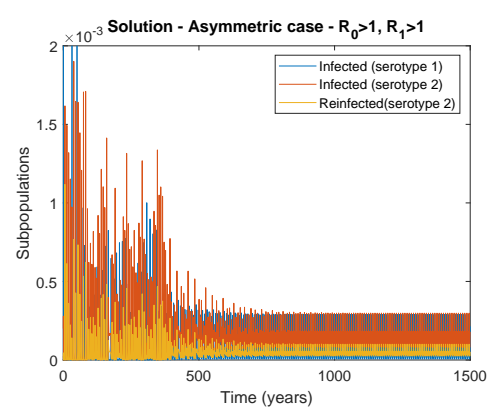

(b) $\phi=0.8$

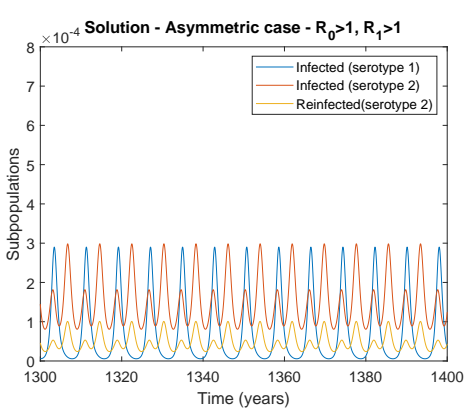

(c) $\phi=0.8$

Figure 6.21: Solutions of the system for $\phi=0.8, \beta_{1}=120, \mathcal{R}_{1}=2.31>1, \beta_{2}=180, \mathcal{R}_{2}=$ $3.46>1$. Initial conditions: $(0.26,0.000032,0.0002,0.0009,0.005,0.05,0.4,0.00001,0.00002)$.

The figures 6.21a to $6.21 \mathrm{c}$ show the solutions of the system at the asymmetric case for the parameter values on the table (3.2) with $\phi=0.8, \beta_{1}=120$ giving $\mathcal{R}_{1}=2.31>1$ and, $\beta_{2}=180$ giving $\mathcal{R}_{2}=3.46>1$. In this case, the long term behaviour shows that the solutions converge to a periodic orbit. 


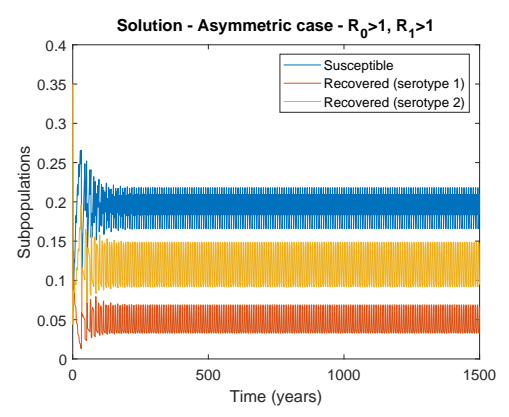

(a) $\phi=2$

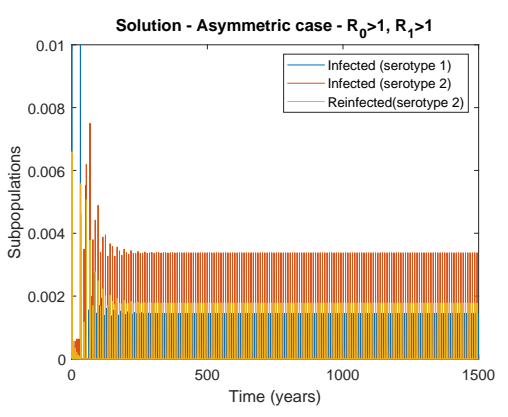

(b) $\phi=2$

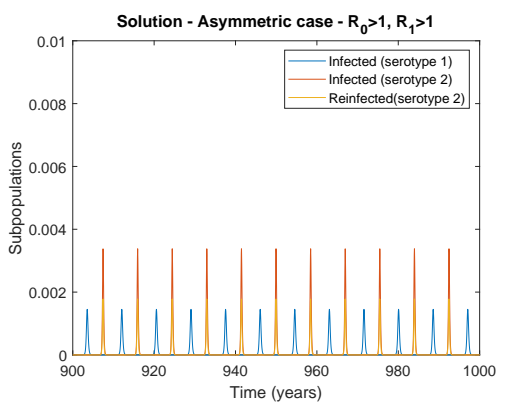

(c) $\phi=2$

Figure 6.22: Solutions of the system for $\phi=2, \beta_{1}=120, \mathcal{R}_{1}=2.31>1, \beta_{2}=180, \mathcal{R}_{2}=$ $3.46>1$. Initial conditions: $(0.25,0.000042,0.0009,0.001,0.01,0.05,0.35,0.00002,0.00003)$.

The figures 6.22a to $6.22 \mathrm{c}$ show the solutions of the system at the asymmetric case for the parameter values on the table (3.2) with $\phi=2, \beta_{1}=120$ giving $\mathcal{R}_{1}=2.31>1$ and, $\beta_{2}=180$ giving $\mathcal{R}_{2}=3.46>1$. In this case, the solutions converge to a periodic orbit.

Although we conclude mathematically that the solution of the system goes to an equilibrium or goes to a periodic orbit, this happens for a long time behaviour. It means it takes much time to get in the asymptotic behaviour. Therefore, biologically speaking, for a short period of time it is hard to predict the next episode of the disease.

\subsection{Main Results}

We can summarize our main results on the table below, where local and global stability of the DFE and of the boundary equilibriums were theoretically proved. While, the local stability and bifurcations of the Coexistence Endemic were numerically showed.

Table 6.1: Stability of the solutions of the delay system: Symmetric parameters

\begin{tabular}{lllll}
\hline Basic R. Number & DFE & Eq. $E_{1}$ & Eq. $E_{2}$ & Int. Equilibrium $E_{3}$ \\
\hline $\mathcal{R}_{1}=\mathcal{R}_{2}$ & & & & \\
\hline $\mathcal{R}_{0} \leq 1$ & GAS & No & No & No \\
$\mathcal{R}_{0}>1$ & Unstable & Unstable & Unstable & LAS for $\phi<\phi_{c}$ \\
& & & & H. B. (stable limit cycle) \\
& & & & Unstable $\phi>\phi_{c}$ \\
\hline
\end{tabular}


Table 6.2: Stability of the solutions of the delay system: Asymmetric parameters

\begin{tabular}{llllll}
\hline Basic R. N. & Invasion N. & DEF & Eq. $E_{1}$ & Eq. $E_{2}$ & Int. Eq. $E_{3}$ \\
\hline $\mathcal{R}_{1}<\mathcal{R}_{2}$ & $\mathcal{R}_{\text {Inv }}$ & & & & \\
\hline $\mathcal{R}_{0} \leq 1$ & $\mathcal{R}_{\text {Inv }}<1$ & GAS & No & No & No \\
$\mathcal{R}_{0}>1$ & $\mathcal{R}_{\text {Inv }}<1$ & Unstable & No $\left(\mathcal{R}_{1}<1\right)$ & GAS & No \\
& & & Unstable $\left(\mathcal{R}_{1}>1\right)$ & & \\
$\mathcal{R}_{0}>1$ & $\mathcal{R}_{\text {Inv }}>1$ & Unstable & Unstable & Unstable & LAS for $\phi<\phi_{c}$ \\
& & & & & H B (stable limit cycle) \\
& & & & & Unstable $\phi>\phi_{c}$ \\
\hline
\end{tabular}

\subsection{Local Analysis of the Endemic Equilibrium of the ODE System}

The analysis of the local stability of the endemic equilibrium (coexistence equilibrium) using the classical theory of the linearization was not successful. We will have to deal with a characteristic polynomial of the a $9 \times 9$ matrix and with the fact that it was not possible to describe the endemic equilibrium in terms of the parameters.

First of all, in order to analyse the stability, we have decided to analyse it numerically (chapter 4). We have found that a Hopf bifurcation occurs for a critical value of the parameter $\phi$ leading to periodic oscillations. The steady state was computed numerically for arbitrary $\phi$ and, as $\phi$ increases, trajectories change from stable endemic coexistence equilibrium to oscillatory dynamics.

Once we have the numerical results in mind, in order to analyse analytically the bifurcation structure we are going to use perturbation theory and symmetry in order to handle the complexity of a direct calculation of the coexistence equilibrium and the eigenvalues.

At the symmetric case, we have already known that since the parameters are symmetric, the dynamic of the model will be also symmetric, in other words, for equal initial conditions, the variables that represent the sub-populations are equal in the respective class for different serotypes. Then, the primary infections are equal, the secondary infections are the same and so on. Thus, we are going to use the symmetry among serotypes, in order to reduce the symmetric system.

First, we analyse the particular case, when $P(t)=e^{-\omega t}$. Now, using the ideas from Billings (2007), we consider that the population reached the equilibrium $N^{*}=\frac{1}{k} \ln \left(\frac{r}{d}\right), r>d$. At the equilibrium, we normalize the ODE, using $\bar{S}=\frac{S}{N^{*}}, \overline{I_{i}}=\frac{I_{i}}{N^{*}}, \overline{I_{i j}}=\frac{I_{i j}}{N^{*}}, \overline{C_{i}}=\frac{C_{i}}{N^{*}}$ and $\overline{R_{i}}=\frac{R_{i}}{N^{*}}$.

Thus, near the equilibrium, at symmetric case, the ODE can be rewritten as 


$$
\begin{aligned}
& \bar{S}^{\prime}(t)=d-d \bar{S}-\beta \bar{S}\left(\overline{I_{1}}+\overline{I_{2}}+\overline{I_{12}}+\overline{I_{21}}\right) \\
& {\overline{I_{1}}}^{\prime}(t)=-(d+\gamma) \overline{I_{1}}+\beta \bar{S}\left(\overline{I_{1}}+\overline{I_{21}}\right) \\
& {\overline{I_{2}}}^{\prime}(t)=-(d+\gamma) \overline{I_{2}}+\beta \bar{S}\left(\overline{I_{2}}+\overline{I_{12}}\right) \\
& {\overline{C_{1}}}^{\prime}(t)=-(d+\omega) \overline{C_{1}}+\gamma \overline{I_{1}} \\
& {\overline{C_{2}}}^{\prime}(t)=-(d+\omega) \overline{C_{2}}+\gamma \overline{I_{2}} \\
& {\overline{R_{1}}}^{\prime}(t)=-d \overline{R_{1}}-\alpha \phi \overline{R_{1}}\left(\overline{I_{2}}+\overline{I_{12}}\right)+\omega \overline{C_{1}} \\
& {\overline{R_{2}}}^{\prime}(t)=-d \overline{R_{2}}-\alpha \phi \overline{R_{2}}\left(\overline{I_{1}}+\overline{I_{21}}\right)+\omega \overline{C_{2}} \\
& {\overline{I_{12}}}^{\prime}(t)=-(d+\gamma) \overline{I_{12}}+\alpha \phi \overline{R_{1}}\left(\overline{I_{2}}+\overline{I_{12}}\right) \\
& {\overline{I_{21}}}^{\prime}(t)=-(d+\gamma) \overline{I_{21}}+\alpha \phi \overline{R_{2}}\left(\overline{I_{1}}+\overline{I_{21}}\right) .
\end{aligned}
$$

Using the symmetry among the serotypes, we reduce the whole system defining new variables

$$
\begin{aligned}
& s=\bar{S}=\frac{S}{N^{*}} \\
& x=\overline{I_{1}}=\overline{I_{2}} \\
& c=\overline{C_{1}}=\overline{C_{2}} \\
& r=\overline{R_{1}}=\overline{R_{2}} \\
& y=\overline{I_{12}}=\overline{I_{21}} .
\end{aligned}
$$

Thus, the endemic equilibrium in the initial system will be the same equilibrium of the following associated reduced model

$$
\begin{aligned}
& s^{\prime}(t)=d-d s-\beta s 2(x+y) \\
& x^{\prime}(t)=-(d+\gamma) x+\beta s(x+y) \\
& c^{\prime}(t)=-(d+\omega) c+\gamma x \\
& r^{\prime}(t)=-\alpha \phi r(x+y)+\omega c-d r \\
& y^{\prime}(t)=-(d+\gamma) y+\alpha \phi r(x+y) .
\end{aligned}
$$

There is still a complexity of the direct calculation of the endemic equilibrium and the eigenvalues, in terms of the dependency of the parameters. Hence, in an attempt to deal with this complexity, we are going to use the perturbation theory.

Note that $d$, the mortality rate, is small compared to the other parameters. This way, we introduce another small parameter $\mu>0$, having now $d$ of the order $\mathcal{O}(\mu)$ and the other parameters of the system in order of $\frac{1}{\mu}$. 
We rescale the parameters in relation to $\mu$, letting $\beta=\frac{\beta_{0}}{\mu}, \alpha=\frac{\alpha_{0}}{\mu}, \omega=\frac{\omega_{0}}{\mu}, \gamma=\frac{\gamma_{0}}{\mu}$, in the sequence, we set the birth rate $\mu$ and the mortality rate $d$. With these parameters set, we are close enough to our original system at the steady state and its behaviour.

As soon as we remove the mortality rate, we are able to estimate the endemic equilibrium analytically, in terms of the parameters and stability. Once we set the mortality parameter $d=0$ (because $d$ is of $\mathcal{O}(\mu))$ it simplifies the model near the equilibrium. Moreover, the mortality term has negligible effect on the steady state because it is a small parameter compared to the other parameters. Then, the resulting local dynamics can be reduced to the following associated system

$$
\begin{aligned}
& s^{\prime}=\mu-\beta s 2(x+y) \\
& x^{\prime}=-\gamma x+\beta s(x+y) \\
& c^{\prime}=\gamma x-\omega c \\
& r^{\prime}=\alpha \phi x r(x+y)+\omega c \\
& y^{\prime}=-\gamma y+\alpha \phi r(x+y) .
\end{aligned}
$$

The qualitatively analysis of this model is an interesting approach of our original system, but it is only valid for small values of the mortality rate and different values of the birth rate, suggesting an attempt to approach the value of the endemic equilibrium, not including the mortality in the long time dynamic and, a possible estimation of the system dynamics and its stability.

The endemic equilibrium of the system (6.24), considering, $x \neq 0$ and $y \neq 0$, in other words, when the disease is already established without the mortality term, now it is easy to calculate. It is giving by

$$
E_{S}=\left(\frac{\gamma_{0}}{2 \beta_{0}}, \frac{\mu^{2}}{2 \gamma_{0}}, \frac{\mu^{2}}{2 \omega_{0}}, \frac{\gamma_{0}}{2 \alpha_{0} \phi}, \frac{\mu^{2}}{2 \gamma_{0}}\right)
$$

Now, we use the linearisation theory to analyse the stability of the endemic equilibrium. The Jacobian matrix of the reduced associated system (6.24) at the steady state $E_{S}$ is given by

$$
J\left(E_{S}\right)=\left[\begin{array}{ccccc}
-\frac{2 \beta_{0} \mu}{\gamma_{0}} & -\frac{\gamma_{0}}{\mu} & 0 & 0 & -\frac{\gamma_{0}}{\mu} \\
\frac{\beta_{0} \mu}{\gamma_{0}} & -\frac{\gamma_{0}}{2 \mu} & 0 & 0 & \frac{\gamma_{0}}{2 \mu} \\
0 & \frac{\gamma_{0}}{\mu} & -\frac{\omega_{0}}{\mu} & 0 & 0 \\
0 & -\frac{\gamma_{0}}{2 \mu} & \frac{\omega_{0}}{\mu} & -\frac{\alpha_{0} \phi \mu}{\gamma_{0}} & -\frac{\gamma_{0}}{2 \mu} \\
0 & \frac{\gamma_{0}}{2 \mu} & 0 & \frac{\alpha_{0} \phi \mu}{\gamma_{0}} & -\frac{\gamma_{0}}{2 \mu}
\end{array}\right]
$$


And, the coefficients of the characteristic polynomial $m(\lambda)=m_{0}+m_{1} \lambda+m_{2} \lambda^{2}+m_{3} \lambda^{3}+m_{4} \lambda^{4}+m_{5} \lambda^{5}$ are given by

$$
\begin{aligned}
& m_{0}=-\frac{2 \alpha_{0} \phi \beta_{0} \omega_{0}}{\mu} \\
& m_{1}=-\frac{\beta_{0} \gamma_{0}^{3} \omega_{0}+2 \alpha_{0} \phi \beta_{0}\left(\gamma_{0}^{2}+2 \gamma_{0} \omega_{0}\right) \mu^{2}}{\gamma_{0}^{2} \mu^{2}} \\
& m_{2}=-\frac{\left(2 \beta_{0}+\alpha_{0} \phi\right) \gamma_{0}^{3} \mu+3\left(2 \beta_{0}+\alpha_{0} \phi\right) \gamma_{0}^{2} \omega \mu+4 \alpha_{0} \phi \beta_{0}\left(\omega_{0}+2 \gamma_{0}\right) \mu^{3}}{2 \gamma_{0}^{2} \mu^{2}} \\
& m_{3}=-\frac{2 \gamma_{0}^{3} \omega_{0}+4 \alpha_{0} \phi \beta_{0} \mu^{4}+\left(6 \beta_{0} \gamma_{0}^{2}+4 \beta_{0} \gamma_{0} \omega_{0}+3 \alpha_{0} \phi \gamma_{0}^{2}+2 \alpha_{0} \phi \gamma_{0} \omega_{0}\right) \mu^{2}}{2 \gamma_{0}^{2} \mu^{2}} \\
& m_{4}=-\frac{\gamma_{0}^{2}\left(\gamma_{0}+\omega_{0}\right) \mu+\left(2 \beta_{0}+\alpha_{0} \phi\right) \gamma_{0} \mu^{3}}{\gamma_{0}^{2} \mu^{2}} \\
& m_{5}=-1 .
\end{aligned}
$$

Since the coefficients of the polynomial are of $\operatorname{order} \mathcal{O}\left(1 / \mu^{2}\right)$ we redefine a polynomial $M(\lambda)=$ $\mu^{2} m(\lambda)$. Thus, we apply the regular perturbation theory, assuming that the solutions of the polynomial $M(\lambda)$ are of the form $\lambda=z_{0}+z_{1} \mu+z_{2} \mu^{2}+\mathcal{O}\left(\mu^{3}\right)$.

Substituting the solutions $\lambda$ in the polynomial $M(\lambda)=\mu^{2} m(\lambda)$ and equalising the terms of the same order we have

$$
\begin{aligned}
& z_{0}=0 \\
& z_{1}=-2 \frac{\alpha_{0} \phi}{\gamma_{0}} \\
& z_{2}=0
\end{aligned}
$$

and,

$$
\begin{aligned}
& z_{0}^{2}=-\beta_{0} \\
& z_{1}=-\frac{1}{2 \gamma_{0} \omega_{0}}\left[\beta_{0} \gamma_{0} \omega_{0}+\alpha_{0} \phi\left(\gamma_{0}-\omega_{0}\right)\right] \\
& z_{2}= \pm v_{0} \sqrt{\beta_{0}} i
\end{aligned}
$$

where $v_{0}=z_{1}\left(2 \beta_{0}\left(\gamma_{0}-\omega_{0}\right)-\alpha_{0} \phi\left(3 \omega_{0}+\gamma_{0}\right)\right)-3 \gamma_{0} \omega_{0} z_{1}^{2}+\frac{\beta_{0}^{2}}{\gamma_{0}}\left(2 \omega_{0}+2 \gamma_{0}\right)-\frac{\beta_{0} \alpha_{0} \phi}{2 \gamma_{0}}\left(\gamma_{0}+6 \omega_{0}\right)$.

Thus, the approximation of the $\mathcal{O}\left(\mu^{3}\right)$ of the eigenvalues are

$$
\lambda_{1}=-2 \frac{\alpha_{0} \phi}{\gamma_{0}} \mu<0
$$

and,

$$
\lambda_{2,3}=-\left(\frac{1}{2 \gamma_{0} \omega_{0}}\left[\beta_{0} \gamma_{0} \omega_{0}+\alpha_{0} \phi\left(\gamma_{0}-\omega_{0}\right)\right]\right) \mu \pm\left(1+v_{0} \mu^{2}\right) \sqrt{\beta_{0}} i
$$


with the negative real part, because $\omega \leq \gamma$, it means that the length of the recovery time is smaller than the length of the cross immunity time, according to the biological references.

The other eigenvalues can be determined by verifying their magnitude, by analysing the coefficients of the characteristic polynomial. Performing this analysis, it is possible to verify that the other solutions of the polynomial $m(\lambda)$ are of the order $\mathcal{O}\left(\frac{1}{\mu}\right)$. Dividing $m(\lambda)$ by the roots found, it can be notice that the real part of the complex roots $\lambda_{4,5}$ is of the form

$$
-\frac{\left(\gamma_{0}+\omega_{0}\right)}{\mu}-\frac{2 \beta_{0} \mu}{\gamma_{0}}+\frac{\left(\beta_{0} \omega_{0}+\alpha_{0} \phi\right) \mu}{\omega_{0}}
$$

Thus, the real part of the eigenvalue is negative since the negative term is of order $\mathcal{O}\left(\frac{1}{\mu}\right)$, whereas the positive term is of the order $\mathcal{O}(\mu)$.

It means it is possible to conclude that the symmetry of the parameters and variables lead to a great reduced system but it is not possible to find the bifurcation structure in the symmetry. Also, with this conditions, we easily verify numerically that for all values of $\phi>0$, the eigenvalues of this reduced associated model are always negative, showing a stable dynamic near the endemic equilibrium, that does not necessarily occur as we have seen numerically in the previous chapter, in the symmetric case for the initial system.

Therefore, in this case we have to deal with the whole system, without the assumption that the variables are symmetric, only that the parameters have the symmetry, since the symmetry among the variables does not reflect the stability of the whole system.

In fact, it is the assumption of the symmetry in the variables and not the perturbation in the mortality term which makes only the stable dynamic appear for the system. We can assure this statement with numerical results of the stability of the system 6.23, at symmetric case, with symmetry in the variables and without the perturbation of the mortality term. It is possible to see that the eigenvalues of Jacobian matrix at the endemic equilibrium of the system (6.23) have always negative real part, independent of the size of the parameter $\phi$ as shown on the following figures. 


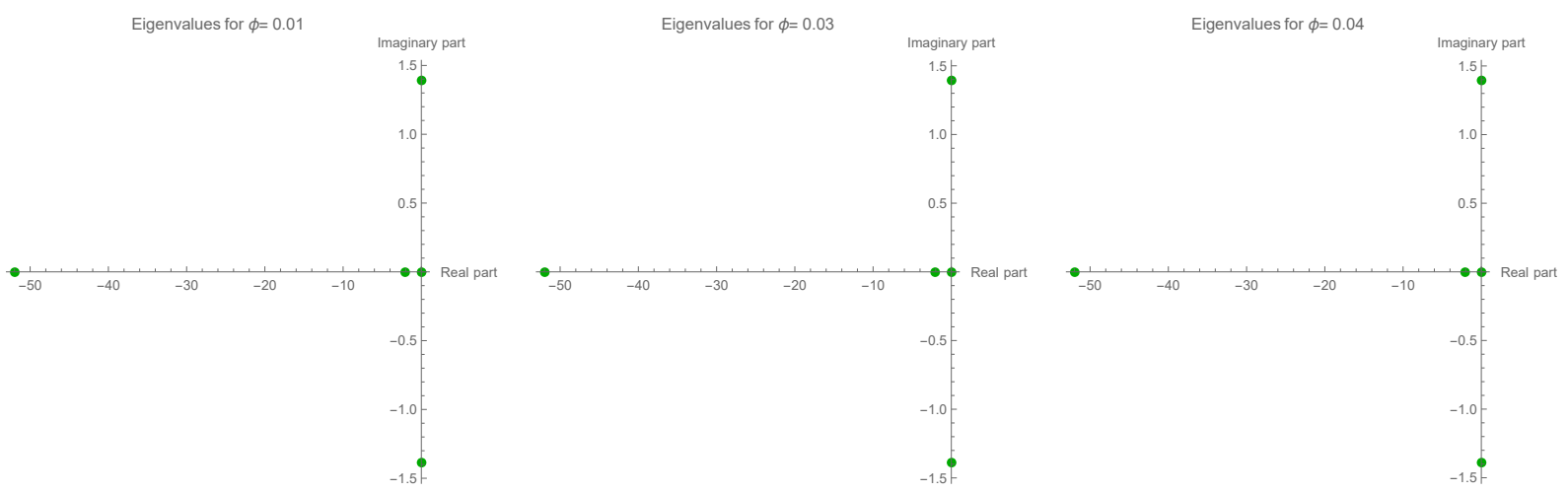
(a) $\phi=0.01$
(b) $\phi=0.03$
(c) $\phi=0.04$

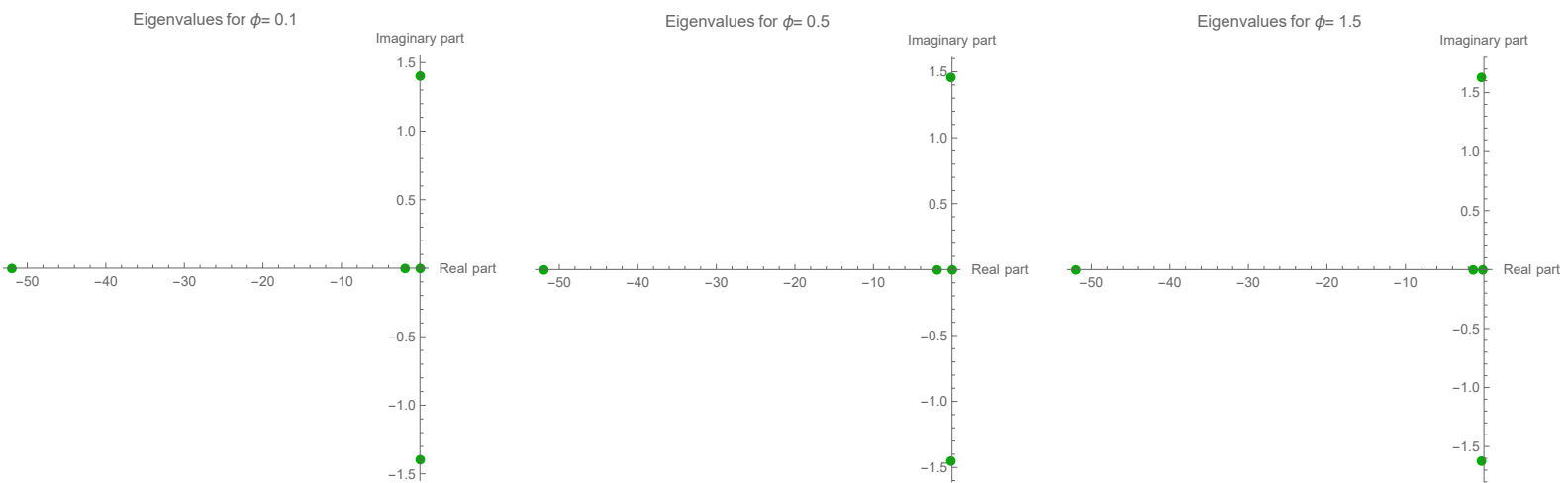

(d) $\phi=0.1$

(e) $\phi=0.5$

(f) $\phi=1.5$

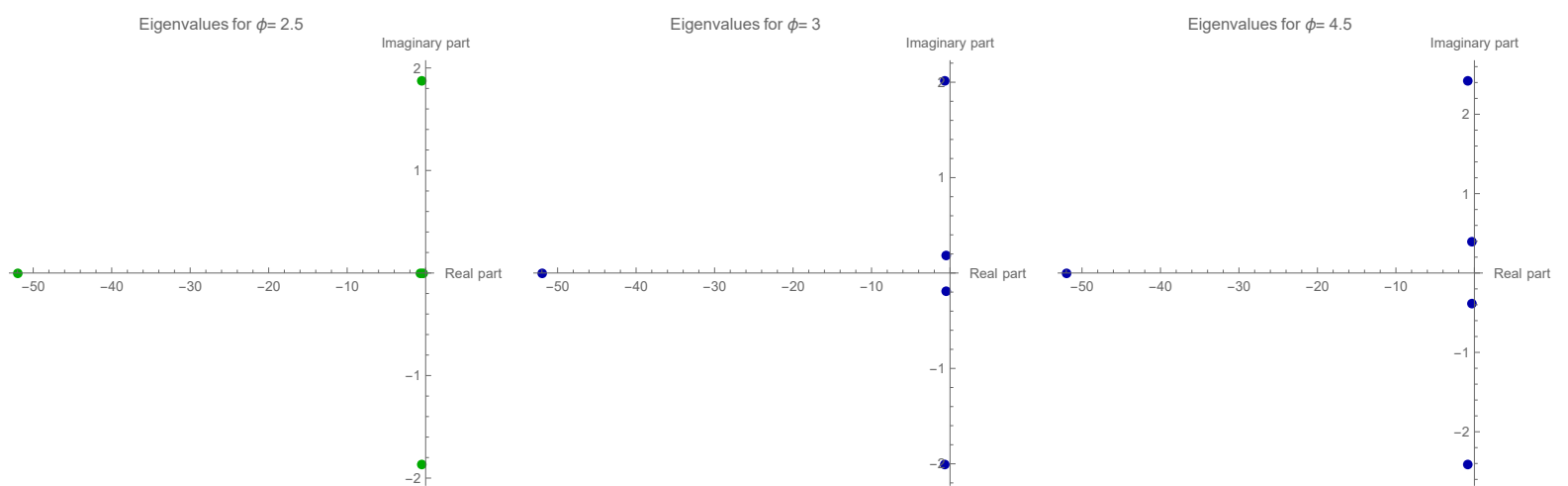
(g) $\phi=2.5$
(h) $\phi=3$
(i) $\phi=4.5$

Figure 6.23: Figures show the eigenvalues of the endemic equilibrium in the complex plane, for each value of $\phi$, at symmetric case, for symmetric system (6.23) ( with symmetry in the variables and without the perturbation in the mortality term). The values used in the simulations are found on table (3.2) with $\beta_{1}=\beta_{2}=180$.

Then, we are going to proceed and work with the whole system 6.21. To simplify, we define new variables as following 


$$
\begin{aligned}
s & =\bar{S}=\frac{S}{N^{*}} \\
x_{i} & =\overline{I_{i}} \\
c_{i} & =\overline{C_{i}} \\
r_{i} & =\overline{R_{i}} \\
y_{1} & =\overline{I_{21}} \\
y_{2} & =\overline{I_{12}} .
\end{aligned}
$$

Thus, the endemic equilibrium in the initial system will be the same equilibrium of the following associated system

$$
\begin{aligned}
& s^{\prime}(t)=d-d s-\beta s\left(x_{1}+x_{2}+y_{1}+y_{2}\right) \\
& x_{1}^{\prime}(t)=-(d+\gamma) x_{1}+\beta s\left(x_{1}+y_{1}\right) \\
& x_{2}^{\prime}(t)=-(d+\gamma) x_{2}+\beta s\left(x_{2}+y_{2}\right) \\
& c_{1}^{\prime}(t)=-(d+\omega) c_{1}+\gamma x_{1} \\
& c_{2}^{\prime}(t)=-(d+\omega) c_{2}+\gamma x_{2} \\
& r_{1}^{\prime}(t)=-\alpha \phi r_{1}\left(x_{2}+y_{2}\right)+\omega c_{1}-d r_{1} \\
& r_{2}^{\prime}(t)=-\alpha \phi r_{2}\left(x_{1}+y_{2}\right)+\omega c_{2}-d r_{2} \\
& y_{1}^{\prime}(t)=-(d+\gamma) y_{1}+\alpha \phi r_{2}\left(x_{1}+y_{1}\right) \\
& y_{2}^{\prime}(t)=-(d+\gamma) y_{2}+\alpha \phi r_{1}\left(x_{2}+y_{2}\right) .
\end{aligned}
$$

Now, we are going to use the same idea used before for the reduced model. We are going to use the perturbation theory. It is important to note that $d$, the mortality rate, is small compared to the other parameters. This way, we introduce another small parameter $\mu$, having now, $d$ is of $\mathcal{O}(\mu)$ and the other parameters of the system in order of $\frac{1}{\mu}$.

We rescale the parameters in relation to $\mu$, letting $\beta=\frac{\beta_{0}}{\mu}, \alpha=\frac{\alpha_{0}}{\mu}, \omega=\frac{\omega_{0}}{\mu}, \gamma=\frac{\gamma_{0}}{\mu}$, in the sequence, we set the birth rate $\mu$ and, the mortality rate $d$. With this parameters set, we are close enough to our original system at the steady state and its behaviour.

As soon as we remove the mortality rate, we are able to estimate the endemic equilibrium analytically, in terms of the parameters and stability. Once we set the mortality parameter $d=0$ (because $d$ is of $\mathcal{O}(\mu))$ it simplifies the model near the equilibrium. Moreover, the mortality term has negligible effect on the steady state because it is a small parameter compared to the other parameters. Then, the resulting local dynamics can be described by the following system 


$$
\begin{aligned}
& s^{\prime}(t)=\mu-\beta s\left(x_{1}+x_{2}+y_{1}+y_{2}\right) \\
& x_{1}^{\prime}(t)=-\gamma x_{1}+\beta s\left(x_{1}+y_{1}\right) \\
& x_{2}^{\prime}(t)=-\gamma x_{2}+\beta s\left(x_{2}+y_{2}\right) \\
& c_{1}^{\prime}(t)=-\omega c_{1}+\gamma x_{1} \\
& c_{2}^{\prime}(t)=-\omega c_{2}+\gamma x_{2} \\
& r_{1}^{\prime}(t)=-\alpha \phi r_{1}\left(x_{2}+y_{2}\right)+\omega c_{1} \\
& r_{2}^{\prime}(t)=-\alpha \phi r_{2}\left(x_{1}+y_{2}\right)+\omega c_{2} \\
& y_{1}^{\prime}(t)=-\gamma y_{1}+\alpha \phi r_{2}\left(x_{1}+y_{1}\right) \\
& y_{2}^{\prime}(t)=-\gamma y_{2}+\alpha \phi r_{1}\left(x_{2}+y_{2}\right) .
\end{aligned}
$$

The qualitatively analysis of this model is an interesting approach of our original system, but it is only valid for small values of the mortality rate and different values of the birth rate, suggesting an attempt to approach the value of the endemic equilibrium, not including the mortality in the long time dynamic and a possible estimation analysis of the Hopf bifurcation structure for values of $\phi$.

The endemic equilibrium of the system (6.38), considering, $x_{i} \neq 0$ and $y_{i} \neq 0$, in other words, when the disease is already established without the mortality term, now it is easy to calculate. And, it is given by

$$
E_{A}=\left(\frac{\gamma_{0}}{2 \beta_{0}}, \frac{\mu^{2}}{2 \gamma_{0}}, \frac{\mu^{2}}{2 \gamma_{0}}, \frac{\mu^{2}}{2 \omega_{0}}, \frac{\mu^{2}}{2 \omega_{0}}, \frac{\gamma_{0}}{2 \alpha_{0} \phi}, \frac{\gamma_{0}}{2 \alpha_{0} \phi}, \frac{\mu^{2}}{2 \gamma_{0}}, \frac{\mu^{2}}{2 \gamma_{0}}\right)
$$

Now, we use the linearisation theory to analyse the stability of the endemic equilibrium. The Jacobian matrix of the reduced associated system 6.38 at the steady state $E_{A}$ is given by

$$
J\left(E_{A}\right)=\left[\begin{array}{ccccccccc}
-\frac{2 \beta_{0} \mu}{\gamma_{0}} & -\frac{\gamma_{0}}{2 \mu} & -\frac{\gamma_{0}}{2 \mu} & 0 & 0 & 0 & 0 & -\frac{\gamma_{0}}{2 \mu} & -\frac{\gamma_{0}}{2 \mu} \\
\frac{\beta_{0} \mu}{\gamma_{0}} & -\frac{\gamma_{0}}{2 \mu} & 0 & 0 & 0 & 0 & 0 & 0 & \frac{\gamma_{0}}{2 \mu} \\
\frac{\beta_{0} \mu}{\gamma_{0}} & 0 & -\frac{\gamma_{0}}{2 \mu} & 0 & 0 & 0 & 0 & \frac{\gamma_{0}}{2 \mu} & 0 \\
0 & \frac{\gamma_{0}}{\mu} & 0 & -\frac{\omega_{0}}{\mu} & 0 & 0 & 0 & 0 & 0 \\
0 & 0 & \frac{\gamma_{0}}{\mu} & 0 & -\frac{\omega_{0}}{\mu} & 0 & 0 & 0 & 0 \\
0 & 0 & -\frac{\gamma_{0}}{2 \mu} & \frac{\omega_{0}}{\mu} & 0 & -\frac{\alpha_{0} \phi \mu}{\gamma_{0}} & 0 & -\frac{\gamma_{0}}{2 \mu} & 0 \\
0 & -\frac{\gamma_{0}}{2 \mu} & 0 & 0 & \frac{\omega_{0}}{\mu} & 0 & -\frac{\alpha_{0} \phi \mu}{\gamma_{0}} & 0 & -\frac{\gamma_{0}}{2 \mu} \\
0 & 0 & \frac{\gamma_{0}}{2 \mu} & 0 & 0 & \frac{\alpha_{0} \phi \mu}{\gamma_{0}} & 0 & -\frac{\gamma_{0}}{2 \mu} & 0 \\
0 & \frac{\gamma_{0}}{2 \mu} & 0 & 0 & 0 & 0 & \frac{\alpha_{0} \phi \mu}{\gamma_{0}} & 0 & -\frac{\gamma_{0}}{2 \mu}
\end{array}\right],
$$

with characteristic polynomial $n(\lambda)=n_{0}+n_{1} \lambda+n_{2} \lambda^{2}+n_{3} \lambda^{3}+n_{4} \lambda^{4}+n_{5} \lambda^{5}+n_{6} \lambda^{6}+n_{7} \lambda^{7}+n_{8} \lambda^{8}+$ $n_{9} \lambda^{9}$ and coefficients given by 


$$
\begin{aligned}
& n_{0}=-\frac{2 \alpha_{0}^{2} \phi^{2} \beta_{0} \omega_{0}^{2}}{\mu^{3}} \\
& n_{1}=-\frac{\alpha_{0} \phi \beta_{0} \omega_{0}\left[\gamma_{0}^{2} \omega_{0}+\alpha_{0} \phi\left(3 \gamma_{0}+7 \omega_{0}\right) \mu^{2}\right]}{\mu^{4}} \\
& n_{2}=-\frac{\alpha_{0} \phi \beta_{0} \gamma_{0}^{2} \omega_{0}\left(3 \gamma_{0}+13 \omega_{0}\right)}{2 \gamma_{0} \mu^{3}}-\frac{\alpha_{0}^{2} \phi^{2}\left(\gamma_{0}^{2} \omega_{0}\left(\gamma_{0}+3 \omega_{0}\right)\right)}{2 \gamma_{0} \mu^{3}}-\frac{\alpha_{0}^{2} \phi^{2} 2 \beta_{0}\left(\gamma_{0}^{2}+12 \gamma_{0} \omega_{0}+10 \omega_{0}^{2}\right) \mu^{2}}{2 \gamma_{0} \mu^{3}} \\
& n_{3}=-\frac{4 \beta_{0} \gamma_{0}^{4} \omega_{0}^{2}+4 \alpha_{0} \phi \gamma_{0}^{4} \omega_{0}^{2}}{\left.4 \gamma_{0}^{2} \mu^{4}\right)}-\frac{20 \alpha_{0}^{2} \phi^{2} \beta_{0} \gamma_{0}^{2}+76 \alpha_{0}^{2} \phi^{2} \beta_{0} \gamma_{0} \omega_{0}+28 \alpha_{0}^{2} \phi^{2} \beta_{0} \omega_{0}^{2}}{4 \gamma_{0}^{2}} \\
& -\frac{\alpha_{0}^{2} \gamma_{0}^{4} \phi^{2}+12 \alpha_{0}^{2} \gamma_{0}^{3} \phi^{2} \omega_{0}+13 \alpha_{0}^{2} \gamma_{0}^{2} \phi^{2} \omega_{0}^{2}+2 \alpha_{0} \phi \beta_{0} \gamma_{0}^{2}\left(\gamma_{0}^{2}+23 \gamma_{0} \omega_{0}+27 \omega_{0}^{2}\right)}{4 \gamma_{0}^{2} \mu^{2}} \\
& n_{4}=-\frac{\mu\left(18 \alpha_{0}^{2} \phi^{2} \beta_{0} \gamma_{0}^{2}+28 \alpha_{0}^{2} \beta_{0} \gamma_{0} \phi^{2} \omega_{0}+4 \alpha_{0}^{2} \beta_{0} \phi^{2} \omega_{0}^{2}\right)}{2 \gamma^{3}}-\frac{4 \beta_{0} \gamma_{0}^{4} \omega_{0}\left(\gamma_{0}+2 \omega_{0}\right)+4 \alpha_{0} \gamma_{0}^{4} \phi \omega_{0}\left(\gamma_{0}+2 \omega_{0}\right)}{2 \gamma^{3} \mu^{3}} \\
& -\frac{3 \alpha_{0}^{2} \gamma_{0}^{4} \phi^{2}+13 \alpha_{0}^{2} \gamma_{0}^{3} \phi^{2} \omega_{0}+6 \alpha_{0}^{2} \gamma_{0}^{2} \phi^{2} \omega_{0}^{2}+2 \alpha_{0} \beta_{0} \gamma_{0}^{2} \phi\left(5 \gamma_{0}^{2}+26 \gamma_{0} \omega_{0}+12 \omega_{0}^{2}\right)}{2 \gamma_{0}^{3} \mu} \\
& n_{5}=-\frac{\gamma_{0}^{2} \omega_{0}^{2}}{\mu^{4}}-\frac{\mu^{2}\left(7 \alpha_{0}^{2} \beta_{0} \gamma_{0} \phi^{2}+4 \alpha_{0}^{2} \beta_{0} \phi^{2} \omega_{0}\right)}{\gamma_{0}^{3}}-\frac{\beta_{0} \gamma^{5}+8 \beta_{0} \gamma_{0}^{4} \omega_{0}+6 \beta_{0} \gamma_{0}^{3} \omega_{0}^{2}+\alpha_{0} \gamma_{0}^{3} \phi\left(\gamma_{0}^{2}+8 \gamma_{0} \omega_{0}+5 \omega_{0}^{2}\right)}{\gamma_{0}^{3} \mu^{2}} \\
& -\frac{50 \alpha_{0} \beta_{0} \gamma_{0}^{3} \phi+96 \alpha_{0} \beta \gamma_{0}^{2} \phi \omega_{0}+16 \alpha_{0} \beta_{0} \gamma_{0} \phi \omega_{0}^{2}+\alpha_{0}^{2} \gamma_{0} \phi^{2}\left(13 \gamma_{0}^{2}+24 \gamma_{0} \omega_{0}+4 \omega_{0}^{2}\right)}{4 \gamma_{0}^{3}} \\
& n_{6}=-\frac{2 \alpha_{0}^{2} \phi^{2} \beta_{0} \mu^{3}}{\gamma_{0}^{3}}-\frac{2 \gamma_{0} \omega_{0}\left(\gamma_{0}+\omega_{0}\right)}{\mu^{3}}-\frac{\mu\left(12 \alpha_{0} \gamma_{0}^{2} \phi+8 \alpha_{0} \phi \beta_{0} \gamma_{0} \omega_{0}+\alpha_{0}^{2} \phi^{2} \gamma_{0}\left(3 \gamma_{0}+2 \omega_{0}\right)\right)}{\gamma_{0}^{3}} \\
& -\frac{4 \beta_{0} \gamma_{0}^{4}+10 \beta_{0} \gamma_{0}^{3} \omega_{0}+2 \beta_{0} \gamma_{0}^{2} \omega^{2}+2 \alpha_{0} \gamma_{0}^{2} \phi\left(2 \gamma_{0}^{2}+5 \gamma_{0} \omega_{0}+\omega_{0}^{2}\right)}{\gamma_{0}^{3} \mu} \\
& n_{7}=-\frac{\gamma_{0}^{4}+\alpha_{0} \phi\left(4 \beta_{0}+\alpha_{0} \phi\right) \mu^{4}+4 \gamma_{0}^{3} \omega_{0}+4 \gamma_{0} \omega_{0}\left(\beta_{0}+\alpha_{0} \phi\right) \mu^{2}+5 \gamma_{0}^{2}\left(\beta_{0}+\alpha_{0} \phi\right) \mu^{2}+\gamma_{0}^{2} \omega_{0}^{2}}{\gamma^{2} \mu^{2}} \\
& n_{8}=-\frac{2\left(\gamma_{0}^{2}+\gamma_{0} \omega_{0}+\left(\beta_{0}+\alpha_{0} \phi\right) \mu^{2}\right)}{\gamma_{0} \mu} \\
& n_{9}=-1 .
\end{aligned}
$$

Our main goal here is to simplify the assumptions as much as possible, in order to get analytical results found in the numerical approach. The reduced model and characteristic polynomial of the reduced model obtained through the symmetry in the variables among the serotypes are a particular case of the model (6.38) and its characteristic polynomial. Since in the reduced model there is no bifurcation structure, we will only work with the quotient polynomial $\frac{m(\lambda)}{n(\lambda)}=r(\lambda)$.

If there is a bifurcation structure, it means that it can only be found in the quotient polynomial 
$r(\lambda)=r_{0}+r_{1} \lambda+r_{2} \lambda^{2}+r_{3} \lambda^{3}+r_{4} \lambda^{4}$, where

$$
\begin{aligned}
& r_{0}=\frac{\alpha_{0} \phi \gamma_{0} \omega_{0}}{\mu^{2}} \\
& r_{1}=\frac{\alpha_{0} \phi\left(\gamma_{0}+3 \omega_{0}\right)}{2 \mu} \\
& r_{2}=\frac{\alpha_{0} \phi\left(3 \gamma_{0}+2 \omega_{0}\right)}{2 \gamma_{0}}+\frac{\gamma_{0} \omega_{0}}{\mu^{2}} \\
& r_{3}=\frac{\gamma_{0}+\omega_{0}}{\mu}+\frac{\alpha_{0} \mu}{\gamma_{0}} \\
& r_{4}=1
\end{aligned}
$$

since, $m(\lambda)$ only show eigenvalues with negative real part.

The coefficients of the polynomial are of $\operatorname{order} \mathcal{O}\left(1 / \mu^{2}\right)$ we redefine a polynomial $R(\lambda)=$ $\mu^{2} r(\lambda)$. Thus, we apply the regular perturbation theory, assuming that the solutions of the polynomial $R(\lambda)$ are of the form $\lambda=z_{0}+z_{1} \mu+z_{2} \mu^{2}+\mathcal{O}\left(\mu^{3}\right)$.

When we substitute the solutions $\lambda$ in the polynomial $R(\lambda)=\mu^{2} r(\lambda)$ and equalise the terms of the same order, we have

$$
\begin{aligned}
z_{0}^{2} & =-\alpha_{0} \phi \\
z_{1} & =\frac{\alpha_{0} \phi}{4 \gamma_{0} \omega_{0}}\left[\gamma_{0}-\omega_{0}\right] \\
z_{2} & = \pm v_{1} \sqrt{\alpha_{0} \phi} i .
\end{aligned}
$$

where $v_{1}=\frac{1}{2 \gamma_{0} \omega_{0} \alpha_{0} \phi}\left[\alpha_{0}^{2} \phi^{2}-\alpha_{0}^{2} \phi^{2}\left(\frac{3 \gamma_{0}+2 \omega_{0}}{2 \gamma_{0}}\right)-\frac{\alpha_{0}^{2} \phi^{2}}{4 \gamma_{0} \omega_{0}}\left(\gamma_{0}-\omega_{0}\right)\left(\frac{5 \gamma_{0}+3 \omega_{0}}{2}\right)\right]$,

Thus, the approximation of the $\mathcal{O}\left(\mu^{3}\right)$ of the eigenvalues are given by

$$
\lambda_{1,2}=\left(\frac{\alpha_{0} \phi}{4 \gamma_{0} \omega_{0}}\left[\gamma_{0}-\omega_{0}\right]\right) \mu \pm\left(1+v_{1} \mu^{2}\right) \sqrt{\alpha_{0} \phi} i
$$

with the positive real part, because $\omega \leq \gamma$, it means that the length of the recovery time is smaller than the length of the cross immunity time, according to the biological references.

The other eigenvalues can be determined by verifying their magnitude, by analysing the coefficients of the characteristic polynomial. Performing this analysis, it is possible to verify that the other solutions of the polynomial $r(\lambda)$ are of the order $\mathcal{O}\left(\frac{1}{\mu}\right)$. When we divide $r(\lambda)$ by the roots found, it can be noticed that the roots are real and of the form

$$
\lambda_{3,4}=-2 \frac{\left(\gamma_{0}+\omega_{0}\right)}{\mu}-\alpha_{0} \phi \mu\left(\frac{1}{\gamma_{0}}+\frac{1}{\omega_{0}}\right) \pm\left(\frac{2}{\mu} \sqrt{\left(\gamma_{0}+\omega_{0}\right)^{2}-\mathcal{O}\left(\mu^{4}\right)}\right) .
$$

The roots are negative since the positive term that appears in one of the eigenvalues, described by the root, is smaller than the negative term of the eigenvalue.

Thus, with the system 6.38, we show that the endemic equilibrium is always unstable. With this analysis, it can be also easy to verify numerically that for all values of $\phi>0$ the eigenvalues of 
the whole system are always negative, except for a pair of complex that has positive real part, showing a unstable dynamic near the endemic equilibrium.

Wherefore, it was not possible to show analytically the bifurcation structure. However, it was possible to prove analytically the instability of the endemic equilibrium. It is what happens for the most values of $\phi$ showed numerically, in the symmetric case.

In this particular case, at symmetric case, for all positive values of $\phi$, there is always a pair of complex eigenvalues that has positive real part, showing that the Coexistence Endemic equilibrium is always unstable, leading to complicated dynamic. 
CHAPTER 7

\section{Discussion and Conclusion}

\subsection{Some Important Considerations}

We have proposed a model with time delay on temporary immunity, allowing general periods of immunity, for instance, $P(t)$ being the probability of a temporarily immune individual which remains immune $t$ units time after entered this state. Thus, we obtain a model to study the spread of Dengue fever in a population, namely a system of Integro-Differential Equations with time delay on the recovery state.

A time delay described by a general distribution is proposed to represent the time that an individual remains temporarily immune for all serotypes, while most articles show a particular function (step function or constant distributed delay) when they want to represent this biological effect.

Also, models the dynamic of total population is always considered constant on those models. Different from that, we have included the Ricker function in order to describe the new individuals born entering the susceptible class. Adding this term, allow us to analyse the dynamics for a long term in order to make them more realistic.

Once we take the total population constant and we assume the probability of remaining in the cross immunity class exponentially distributed and equal for different serotypes, consequently, our model is very similar to the ODE models of the Aguiar (2007) and Aguiar (2008). However, it is important to highlight the difference since we assume different assumption about the ADE effect.

The model that Aguiar (2007) and Aguiar (2008) have proposed was an ODE system for two strains, without vector dynamics and with constant population. The ADE effect was described by a 
constant rate $\phi$ and, the temporarily immune class was added on the model with the assumption that the infection gives a temporary protection for all serotypes no matter which strain the individual was infected and, it is the same for different strains.

In that work, the temporary immune class is represented by $R_{i}$, while the recovery class is $S_{i} . \quad \frac{1}{\alpha}$ is the average time immunity and, $\mu$ the constant birth rate that is equal to death constant rate. The assumption for ADE effect is related to a decreasing (or increasing) of the transmissibility. While this assumption is taken by Aguiar (2007), the same constant rate on our model is used to describe the increasing probability to the reinfection, in other words, an increasing of the susceptibility of the recovered individual.

In the study by Aguiar (2008), numerical analysis were made and deterministic chaos could be observed, with the immunity constant rate $\alpha$ being two months and, for $\phi<1$, which indicates that with the severe disease the patients stay hospitalized and it implies that less transmissions occur.

With respect to analytic theory in the study done by Aguiar (2008), concerning to the symmetric case, the stationary states were obtained when the two strains were equal. They have described analytically the coexistence strains solution and the solution for the extinction of one of them, for the symmetric case only.

For asymmetric case, numerical analysis were made by Kooi (2014). It was observed the dependency of the parameter $\phi$ for ADE effect, also the dependency of parameter $\epsilon$, which describes the difference among the infection rates. Bifurcations diagrams are presented and calculated by numerical simulations. Also, analytical stability analysis were described, for asymmetric case, calculating the stability of the Disease Free Equilibrium (DFE) and for the Boundary equilibrium only.

It was found that the stability of DFE depends on the sign of two eigenvalues and, from this threshold value, they define the Basic Reproduction Number $\mathcal{R}_{0}$. While for the stability of Boundary equilibrium the author affirms that just one single eigenvalue can change under positive parameter changes and, that condition determines the bifurcation saw on the bifurcations numerical diagrams (Kooi, 2014).

There is no mention of a coexistence equilibrium for the asymmetric case over the studies mentioned above, for the ODE system. The analytical result for coexistence equilibrium and the stability were obtained only for the symmetric case by Aguiar (2008).

Although no coexistence equilibrium was obtained for the asymmetric case, in the works by Aguiar (2007, 2008); Kooi (2014) for similar ODE system, in our work even for the ODE model (particular case) we proved that for certain values of the parameter that represent the enhancement, there is indeed an endemic equilibrium (or coexistence equilibrium), within the region, which is a very important result because, according to VinodKumar (2013), different serotypes have been co-circulating in the same area with one of them being dominant during an outbreak. 
Also, we proved numerically that only for small parameter values that represent the ADE the equilibrium can be stable. For all the cases, symmetric and asymmetric, it was showed that bifurcation occurs and, for the most of the values of the $\phi$ the equilibriums exist, but it is unstable. Thus, the coexistence of the two serotypes is possible but not established, the solution oscillates and the serotypes begin to compete for the same resource.

Similar results were found by Esteva (2003). They proposed a simpler model for population and the dynamic of the disease. However, they could incorporate the dynamic of the vector population (mosquito population).

The dynamic of the vector population in the study by Esteva (2003) is simple: two serotypes can infect susceptible vector and, once they are infected, they do not recover from the disease. Humans population dynamics were divided by susceptible state, infected, recovered and reinfected, in a dynamic with two different serotypes and different probability of infection and recover. In addition, over the work, different constant parameter was added to represent either cross immunity protection, if $\sigma$ is smaller than one, or increasing susceptibility to other serotype, when $\sigma>1$.

With this model, Esteva (2003) found that there are four equilibriums of the system, the DFE, two boundary equilibriums and the coexistence equilibrium, even in the asymmetric case. In this case, they affirm that coexistence of both serotypes is possible for large range of parameters. However, this estimative of the stability of the endemic equilibrium was showed only by numerical analysis.

It is important to emphasize that Esteva (2003) included vector population in the dynamics. However they do not differentiate the cross immunity protection and the ADE effect, it means that both biological consequences cannot happen together, either it is partial protection or enhancement of the susceptibility. We differentiate these two process adding a cross immunity time class as Aguiar (2007) and using the hypotheses about the increasing of the susceptibility for the reinfection, representing the ADE consequence, as Esteva (2003).

Although these highlighted differences, we have found similar results as the existence of the coexistence equilibrium of the system. However, while our results show oscillatory dynamic and unstable dynamics for almost all the values of the ADE, Esteva (2003) could prove numerically that for all values of the enhancement the coexistence serotype is stable, only having competitive exclusion when there is no reinfection.

Therefore, we can indeed remove the dynamic of the vectors and add more characteristics of the disease in the human dynamics, including a general time for cross immunity protection, different values for recovery and different values of infectivity resulting in a complex analysis. As soon as we remove the vector dynamic, we are able to achieve the mathematical and biological results which were not obtained previously, and may not have as much impact as if we are more interesting in the analysis of the infected human population and the impact of the diseases in it, since without vectors we could find 
similar analysis regarding the existence of solutions.

Concerning to the dynamic behaviour, our model brings more robust and sophisticated results than the model of the Esteva (2003). Competitive exclusion is presented in the model of Esteva (2003) only in the case when there is no reinfection, however our model shows complex dynamics, more reliable with the reality and taking into account the Competitive Exclusion Principle.

\subsubsection{The Competitive Exclusion Principle}

The Competitive Exclusion Principle states that in the basic ODE system with multiple strains for disease or in case of having different species competing, only the strain (or specie) which has the biggest Reproduction Number persists (if it is larger than one), the other ones die out. The coexistence of strains or different species are not possible unless in the symmetric scenario when the Basic Reproduction Number of the different strains are equal.

According to Martcheva (2015) the principle was first formulated as a law in 1930 by an ecologist Georgy Gause through a laboratory experiment. It was stated that competitors in one niche cannot coexist and, consequently, the other species become extinct.

Bremermann (1989) has modified a host parasite model proposed by Anderson and May and postulated a condition which the parasite persists in host population and validate the principle also for this case. For time delay systems, the principle was derived by Cai (2013) under a stronger condition. In both papers, the models do not consider the possibility of an individual being infected twice in different occasions.

In our model, it is noticed the occurrence of a coexistence equilibrium also for asymmetric case, as in the works by Esteva (2003) and Martcheva (2015), whose stability depends on the force of the enhancement. The mechanism used in our model that led to the occurrence of an equilibrium with coexistence strains was the epidemiological consequence: cross immunity and the biological assumption that different serotypes can infect a host again after the cross immunity period.

In fact, Martcheva (2015) cites and gives examples of some mechanisms that have been identified as causing coexistence of pathogens, such as mutation virus, superinfection (individual with strain one becomes infected with strain two), co-infection and cross immunity (cross immunity means, according to this author, that the other infection occurs after recovered from the other), which is the case in our model.

The possibility of the system exhibits the coexistence equilibrium depends on intrinsically of the values of the parameters that may not be fiscally and biologically feasible, which mean that the principle is validated in this case.

However, the principle was postulated for models that do not permit the reinfection in different occasions which means that this different mechanism may be led to exhibit the coexistence equilibrium. 
Thus, for biological feasible parameters values, the endemic equilibrium could exist. Although it will be always unstable, leading to complex dynamic where sometimes, the infection one resists leading to the other can be almost extinct and sometimes, the opposite. For a long term behaviour these switches between the strains seem to be an important result.

According to Martcheva (2015), there are other mechanisms known to induce coexistence of multiple pathogens, however what causes this coexistence is still an open question. This model carrying these mechanisms which can cause the coexistence of pathogens, as a consequence, these switches among the strains, have an important role, once some diseases still considered a public health problem, as Dengue fever, have this multi-strain interaction characteristic, where different strains co-circulate in a region.

\subsubsection{The Invasion Reproduction Number}

It is usual to find another important threshold value when working with multiple strain model. In this case, when we have the coexistence equilibrium, it is normally easy to find a threshold value that gives the stability of this equilibrium. In our model we called $\mathcal{R}_{I n v}$ and this value determines the stability of the Boundary equilibrium, as well as it is a threshold for the endemic equilibrium be located in the biological positive region.

This quantity found is called Invasion Reproduction Number (of strain one at the equilibrium of strain two). Martcheva (2015) denoted this value by $\mathcal{R}_{1}^{2}$ and it is defined epidemiologically as a number of secondary infections that one individual infected with the strain one will produce in a population, in which the strain two is at equilibrium during its infectious lifetime.

This Invasion Reproduction Number is very important to determine whether a strain can invade another. Thus, when $\mathcal{R}_{1}^{2}=\mathcal{R}_{\text {Inv }}<1$ strain one cannot grow when strain two is at equilibrium. However, if $\mathcal{R}_{1}^{2}=\mathcal{R}_{\text {Inv }}>1$ both strains grow and begin to coexist.

\subsection{Final Conclusions}

In this work we have carried out a mathematical model, namely an Integro-differential equations system, which can be applied to study a multi strain infectious disease, motivated by Dengue fever. Dengue fever is endemic in more than 100 countries WHO (2018) and it remains a major public health problem.

Over the years, some mathematical models for infectious diseases and specially for Dengue fever have been developed, most of them, in the format of ODEs. However, epidemics propagation are not instantaneous and it is more appropriate to model epidemics incorporating continuously distributed delay and not discrete delay or constant time. 
Therefore, by considering Ricker function to describe susceptible newborns, a distributed delay to describe a temporary immunity and a constant parameter to describe the ADE effect, we analyse a multi serotype epidemic model in order to understand the transmission and the effects of the spread of the disease in a population. Additionally, we aim to analyse the effect of time delay and the ADE effect in the system.

In the chapter 2, a general form for the temporary immunity time was considered. Time delay was used to model phenomena that an individual may not be immediately recover after being infected. Using results from IDE systems, we are also capable to prove the existence of four equilibriums and the stability of the equilibriums in both cases, symmetric and asymmetric.

We proved the existence of the DFE, the two boundary equilibriums and the Coexistence Endemic equilibrium, under some conditions. We showed that the disease will die out if the Reproduction number is smaller than 1. If the Invasion Reproduction number is smaller than 1, one strain dies out and the other will persist. And, in the last scenario the two strains will coexist if the Invasion Reproduction number is bigger than 1 . The existence of the endemic equilibrium was also theoretically proved even for the asymmetric case.

Lyapunov functions can be an effective tool for proving global stability of IDE systems. Thus, we could prove the global stability of DFE and the boundary equilibrium, in the case that the Invasion number is smaller than one.

We also showed numerically the stability of the Coexistence endemic equilibrium, showing stability for small values of the parameter representing ADE, while the equilibrium will be unstable for bigger values. The possibility for Hopf bifurcation was allowed only through a numerical analysis by considering the parameter representing ADE effect as a bifurcation parameter. This kind of bifurcation is local, thus only for values close to the bifurcation critical point we can conclude that a limit cycle exists, therefore the solutions show periodic behaviour. Moreover, our results showed that incorporating distributed delays would arise periodic solutions and show instability of the endemic equilibrium for the most values of the bifurcation parameter.

Solutions of the system were performed over chapter 3 showing oscillatory dynamics. Although the solutions of the system, for values further from bifurcation critical value, either go to an equilibrium or go to a periodic orbit, this only happens for a long time behaviour, taking quite long time to reach the asymptotic behaviour. Therefore, for a short period, the behaviour of the solutions is unknown and hard to predict.

Over chapter 4, we have shown that direct calculation of the equilibrium, its eigenvalues and the stability of the Coexistence equilibrium can be only addressed by numerical calculations. Relaxing assumptions in the symmetric case can lead to a great reduced system. For instance, simplifying the symmetric case and assuming symmetry in the variables lead us to a five equations system. Even though 
it has the same equilibriums, the system with symmetry in the variables does not reflect the stability of the endemic equilibrium of the whole system, as we have seen numerically. Although, we could verify and conclude that the bifurcation and instability does not occur in the symmetric manifold.

Whereas the assumption of the perturbation in the mortality term allows us to have an analytic form of the endemic equilibrium with neglected mortality term. Besides, it allows us to show the eigenvalues, likewise the instability of the Coexistence equilibrium. However, this assumption did not reflect the bifurcation structure seen in the numerical analysis. Hence, we could verify the instability direction is out of the symmetric manifold, on the other hand, the Hopf bifurcation already passed with this perturbation.

We finally conclude this work by adding the mortality rate caused by disease. By adding a small mortality in the original system, from biological point of view, it could represent better the dynamic of Dengue fever. However, from the point of view of the qualitative study, through mathematical tools, the behaviour of both models is similar because of the continuity in the parameter. Therefore, the original system, without the mortality for disease, can be a good representation for the study of the propagation of the disease and it brings the advantage of being a simpler model.

A special form for the temporary immunity function was considered in the original model, over the chapter 6. By considering the temporary immunity time exponential distributed, it was able to reduce the IDE system to an ODE system. This way, we have more resources to work with, mathematically speaking. Moreover, qualitative analysis of the ODE system gave us a visual picture of the dynamic behaviour.

Comparing the particular case (exponential distributed function) with the general case, the qualitative behaviour of the system was not altered by the choice of function. However, the Invasion number depends on the average cross protection time, which can affect whether the infection could coexist.

Lastly, mathematical models were used in order to focus on understanding the spread of infectious disease as well as suggesting interventions to control or even predicting the consequences of the propagation. With this model, we tried to explain why it is so hard to predict Dengue fever, and we concluded that there are some mechanism and intrinsic characteristics of Dengue fever, such as ADE and cross protection, which may hinder the prediction of the next outbreaks of the disease. 


\section{Appendix}

\section{Invariant Region for ODE System}

For biological reasons we look for steady states of the ODE system that belong to a region of the $\mathbb{R}_{+}$. In this way, each input will be positive which is biological reasonable since we are dealing with variables that represent sub-population classes. We are going to prove that this region $\Omega=$ $\left\{\left(S, I_{1}, I_{2}, C_{1}, C_{2}, R_{1}, R_{2}, I_{12}, I_{21}, R\right) \in \mathbb{R}_{+}^{10}\right.$ such that $S+I_{1}+I_{2}+C_{1}+C_{2}+R_{1}+R_{2}+I_{12}+I_{21}+R \leq$ $\left.N^{*}\right\}$, described before, it is indeed a positive invariant set for the system (1.8), then all solution with initial condition at $\Omega$ will remain in $\Omega$.

Theorem 16. The $\Omega=\left\{\left(S, I_{1}, I_{2}, C_{1}, C_{2}, R_{1}, R_{2}, I_{12}, I_{21}, R\right) \in \mathbb{R}_{+}^{10}\right.$ such that $S+I_{1}+I_{2}+C_{1}+$ $\left.C_{2}+R_{1}+R_{2}+I_{12}+I_{21}+R \leq N^{*}\right\}$, region is positively invariant set for the system (1.8).

Proof. In fact, it can be easily verified that solutions of the system (1.8) with non-negative initial conditions remain non-negative. In particular, for $S$, we assume that $\tau>0$ is the first time such that $S(\tau)=0$. Then, at $S(\tau)=0$, for the first equation of the system we have

$$
\frac{d S}{d t}=d N^{*}>0
$$

This means that $S(t)<0$ for $t \in(\tau-\epsilon, \tau)$. Therefore $S(t)>0$, for $t \geq 0$.

Also, at $S=N^{*}$

$$
\frac{d S}{d t} \leq 0
$$

then $S(t) \leq N^{*}$ for $t \geq 0$. 
Now, adding the equations of the system (1.8) we have

$$
\begin{aligned}
& \frac{d S}{d t}+\frac{d I_{1}}{d t}+\frac{d I_{2}}{d t}+\frac{d C_{1}}{d t}+\frac{d C_{2}}{d t}+\frac{d R_{1}}{d t}+\frac{d R_{2}}{d t}+\frac{d I_{12}}{d t}+\frac{d I_{21}}{d t}+\frac{d R}{d t}= \\
& d N^{*}-d\left(S+I_{1}+I_{2}+C_{1}+C_{2}+R_{1}+R_{2}+I_{12}+I_{21}+R\right)
\end{aligned}
$$

Then

$\lim \sup _{t \rightarrow \infty} S(t)+I_{1}(t)+I_{2}(t)+C_{1}(t)+C_{2}(t)+R_{1}(t)+R_{2}(t)+I_{12}(t)+I_{21}(t)+R(t) \leq N^{*}$.

This shows that any trajectory $p(t)=\left(s(t), i_{1}(t), i_{2}(t), c_{1}(t), c_{2}(t), r_{1}(t), r_{2}(t), j_{1}(t), j_{2}(t), r(t)\right)$ with the initial condition in $\Omega$, remains in $\Omega$ for all $t$.

\section{Results from Fred Brauer}

This following result was used in order to reach the corollarys about the stability of the solutions of the Time Delay system. This result is from Fred Brauer in his work from 1978, which was found in Brauer (1978).

It is considered a Integro-differential system of the form

$$
x^{\prime}(t)=H(t)+F\left[x(t), \int_{0}^{\infty} g(x(t-s)) P(s) d s\right],
$$

where $H(t)$ tends to zero sufficiently rapidly as $t \rightarrow \infty$. Regarding $H$ as a perturbation, it is considered the unperturbed system

$$
x^{\prime}(t)=F\left[x(t), \int_{0}^{\infty} g(x(t-s)) P(s) d s\right] .
$$

If $\int_{0}^{\infty} P(s) d s<\infty$ we can define an equilibrium to be a solution $x_{\infty}$ of the equation

$$
F\left[x_{\infty}, y_{\infty}\right]=F\left[x_{\infty}, g\left(x_{\infty}\right) \int_{0}^{\infty} P(s) d s\right]=0
$$

Let $u(t)=x(t)-x_{\infty}$ then we write $(7.4)$ as

$$
u^{\prime}(t)=F_{x}\left(x_{\infty}, y_{\infty}\right) u(t)+F_{y}\left(x_{\infty}, y_{\infty}\right) g^{\prime}\left(x_{\infty}\right) \int_{0}^{\infty} u(t-s) P(s) d s+\psi(u(t))
$$

$\psi$ denotes the function with the high order in the variables.

Then, the asymptotic stability of the solution $u=0$ of the (7.6) and, hence of the $x=x_{\infty}$ of (7.4) (Grossman, 1970; Miller, 1972), is a consequence of the uniform asymptotic stability of the solution $u=0$ of the linear system (7.6) (without the function $\psi$ ), in other words,

$$
u^{\prime}(t)=F_{x}\left(x_{\infty}, y_{\infty}\right) u(t)+F_{y}\left(x_{\infty}, y_{\infty}\right) g^{\prime}\left(x_{\infty}\right) \int_{0}^{\infty} u(t-s) P(s) d s
$$


It is known by Grossman (1973) it remains true if an integrable function is added to the right side of 7.6. If $u(t)$ is given and bounded, also if $\int_{0}^{\infty} s P(s) d s<\infty$, the function

$$
H(t)=\int_{t}^{\infty} u(t-s) P(s) d s
$$

is integrable. These results are summarized and formalized in the follow theorem.

Theorem 17. Theorem 2 (Section 3) (Brauer, 1978). Let $x_{\infty}$ be an equilibrium of (7.4), and suppose

$$
\begin{aligned}
& \int_{0}^{\infty} H(s) d s<\infty \\
& \int_{0}^{\infty} P(s) d s<\infty \\
& \int_{0}^{\infty} s P(s) d s<\infty .
\end{aligned}
$$

If the solution $u=0$ of the (7.7) is uniformly asymptotically stable, then the equilibrium $x_{\infty}$ of the (7.3) is uniformly asymptotically stable.

\section{The Invariance Principle}

The Invariance principle used in chapter 3, in order to show global stability through Lyapunov function is a extension of the LaSalle's Invariance Principle for ODE (LaSalle, 1976). The Invariance principle for IDE, definition of Lyapunov function and details can be found in LaSalle (1976) and Burton (2005).

Theorem 18. (LaSalle's Invariance Principle) (LaSalle, 1976) Let $\Omega \subset D$ be a compact set that is positively invariant with respect to the system $x^{\prime}=f(x)$. Let $L: D \rightarrow \mathbb{R}$ be a continuously differentiable function such that $L^{\prime}(x) \leq 0, \forall x \in \Omega$. Let $E$ be the set of all points in $\Omega$ where $L^{\prime}(x)=0$. Let $Y$ be the largest invariant set in $E$. Then every solution starting in $\Omega$ approaches $Y$ as $t$ goes to infinity. 


\section{Bibliography}

Adams, B., Boots, M. Modelling the relationship between antibody-dependent enhancement and immunological distance with application to dengue. Journal of Theoretical Biology. 242: 337-346 (2006).

Aguiar, M., Stollenwerk, N. A new chaotic attractor in a basic multi-strain epidemiological model with temporary cross-immunity. arXiv: 0704.3174v1 (2007).

Aguiar, M., Kooi, B., Stollenwerk, N. Epidemiology of Dengue Fever: A model with temporary cross immunity and possibly secondary infection shows bifurcations and chaotic behaviors in wide parameter region. Math. Model. Nat. Phenom. Vol 3 (4): 48-70 (2008).

Andraud, M., Hens, N., Marais, C., Beutels, P. Dynamic Epidemiological Models for Dengue Transmission: A Systematic Review of Structural Approaches. PLoS ONE. Vol 7(11): e49085 (2012).

Bianco, S., Shaw L. B., Schwartz I. B. Epidemics with multistrain interactions: The interplay between cross immunity and antibody-dependent enhancement. Chaos. Vol 19: 043123 (2009).

Billings, L. et al. Instabilities in multiserotype disease models with antibody-dependent enhancement. Journal of Theo. Bio. 246: 18-27 (2007).

Brauer, F. Perturbations of Nonlinear Systems of Differential Equations. Journal of Mathematical Analysis and Applications. 14: 198-206 (1966).

Brauer, F. A Nonlinear Variation of Constants Formula for Volterra Equations. Math. Systems Theory. 6: 226-234 (1972). 
Brauer, F. Asymptotic Stability of a Class of Integro-differential equation. Journal of Differential Equation. 28: 180-188 (1978).

Brauer, F. et al. Mathematical Epidemiology. Springer, 2008.

Bremermann, H. J., Thieme, H. R. A Competitive exclusion principle for pathogen virulence. J. Math. Biol. 27: 179-190 (1989).

Burton, T. A. Volterra Integral and Differential Equations 2 ed. Math. Sci. Eng. Elsevier, 2005.

Cai, L., Martcheva, M., Li, X. Competitive exclusion in a vector host epidemic model with distributed delay. Journal of Biological Dynamics. Vol 7 (1) : 47-67 (2013).

Chen, D., Xu, Z. Global dynamics of a delayed diffusive two-strain disease model. Differential Equations and Applications. Vol 8 (1): 99-122 (2016).

Cooke, K. L., Yorke, J. A. Some equations modelling growth processes and gonorrhea epidemics. Math. Biosci. Vol 16 (1973).

Cooke, K. L., Van den Driessche, P. Analysis of an SEIRS epidemic model with two delays. J. Math. Biol. 35: 240-260 (1996).

Cooke, K., Van den Driessche, P., Zou, X. Interaction of maturation delay and nonlinear birth in population and epidemic models. J. Math. Biol. 39: 332-352 (1999).

Domoshnitsky, A., Goltser Ya. M. Approach to Study of Bifurcations and Stability of Integro-Differential Equations. Mathematical and Computer Modelling 36: 663-678 (2002).

Edelstein-Keshet, L. Mathematical Models in Biology. SIAM. PA, USA. 2005.

Esteva, L., Vargas, C. Coexistence of different serotypes of dengue virus. J. Math. Biol. 46: 31-47 (2003).

Feng, X., Wang, K., Zhang, F., Teng, Z. Threshold dynamics of a nonlinear multigroup epidemic model with two infinite distributed delays. Math. Meth. Appl. Sci. Vol 40 (7) (2016).

Ferguson, N., Anderson, R., Gupta S. The effect of antibody-dependent enhancement on the transmission dynamics and persistence of multiple strain pathogens. Proc. Natl. Acad. Sci. Vol 96 (2): 790-794 (1999).

Ferguson, N. M., Donnelly, C. A., Anderson, R. M. Transmission dynamics and epidemiology of dengue: insights from age-stratified sero-prevalence surveys. Philosophical Transactions of the Royal Society B: Biological Sciences. Vol 354(1384): 757-768 (1999). 
Grossman, S. I., Miller, R. K. Perturbation theory for Volterra Integro-differential systems. Journal of Differential Equations. Vol 8: 457-474 (1970).

Grossman, S. I., Miller, R. K. Nonlinear Volterra Integro-differential systems with L^1-Kernels. Journal of Differential Equations. Vol 13: 551-556 (1973).

Guan, J., Wu, L., Chen, M., Dong, X., Tang, H., Chen, Z. The stability and Hopf Bifurcation of the Dengue Fever model with time delay. Italian Journal of Pure and Applied Mathematics. 37 : 139-156 (2017).

Gubler, D. J. et al. (Ed.). Dengue and dengue hemorrhagic fever. CABI, 2014.

Guzman, M. G., Alvarez, M., Halstead, S. B. Secondary infection as a risk factor for dengue hemorrhagic fever/dengue shock syndrome: an historical perspective and role of antibody-dependent enhancement of infection. Archives of Virology. 158: 1445-1459 (2013).

Hethcote, H. K., Stech, H. W., Van den Driessche, P. Nonlinear Oscillations in Epidemic Models. SIAM J. Appl. Math. Vol 40 (1) (1981).

Hethcote, H. W., Lewis, M. A., Van den Driessche, P. An epidemiological model with a delay and a nonlinear incidence rate. J. Math. Biology. 27: 49-64 (1989).

$\mathrm{Hu}, \mathrm{K}$. et al. The effect of antibody-dependent enhancement, cross immunity, and vector population on the dynamics of dengue fever. Journal of Theoretical Biology. 319: 62-74 (2013).

Huang, C. Cao, J. Wen, F. Yang, X. Stability Analysis of SIR Model with Distributed Delay on Complex Networks. PLoS ONE. Vol 11(8): e0158813 (2016).

IBGE/DPE/ Coordenação de População e Indicadores Sociais. Gerência de Estudos e Análises da Dinâmica Demográfica. http://tabnet.datasus.gov.br/cgi/idb2012/a11tb.htm Accessed in December, 2018.

Johansson, M., Hombach, J., Cummings, D. A. T. Models of the impact of dengue vaccines: A review of current research and potential approaches. Vaccine. 29: 5860-5868 (2011).

Kooi, B. W., Aguiar, M., Stollenwerk, N. Analysis of an asymmetric two-strain dengue model. Math. Biosciences. 248: 128:139 (2014).

LaSalle J. P. The Stability of Dynamical Systems. In Regional Conference Series in Applied Mathematics. SIAM, USA (1976). 
Li, M. Y., Shuai, Z., Wang, C. Global stability of multi-group epidemic models with distributed delay. J. Math. Anal. Appl. 361: 38-47 (2010).

Lynch, S. Dynamical systems with applications using MATLAB. Springer, 2004.

Maier, S. B., Huanga, X., Massad, E., Amaku, M., Burattini M. N., Greenhalgh, D. Analysis of the optimal vaccination age for dengue in Brazil with a tetravalent dengue vaccine. Mathematical Biosciences 294: 15-32 (2017).

Massad, E., Coutinho, F.A., Burattini M.N., Lopez L.F. The risk of yellow fever in a dengue-infested area. Trans. R. Soc. Trop. Med. Hyg. 95(4): 370-374 (2001).

Martcheva, M. Introduction to Mathematical Epidemiology. Vol 61. New York: Springer, 2015.

Miller, R. K. Nonlinear Volterra Integral Equations. W. A. Benjamin, Inc. 1971.

Miller, R. K. Asymptotic stability properties of linear Volterra Integro-differential equations. Journal of Differential Equations. 10: 485-506 (1972).

Mustafa, M. S., Rasotgi, V., Jain, S., Gupta, V. Discovery of fifth serotype of dengue virus (DENV-5): A new public health dilemma in dengue control. Medical Journal Armed Forces India. Vol 71 (1) : 67-70 (2015).

Murray, J. D. Mathematical Biology I. An Introduction. 3rd ed. Springer. 2002.

Nah, K., Nakata, Y., Röst, G. Malaria dynamics with long incubation period in host. Computers and Mathematics with Applications. Vol 68: 915-930 (2014).

O’ Regan, S. M., Kelly, T. C., Korobeinikov, A., O’Callaghan, M. J. A., Pokrovskiia, A. V. Lyapunov functions for SIR and SIRS epidemic models. Applied Mathematics Letters. Vol 23 (4): 446-448 (2010).

Reich, N.G., Shrestha, S., King, A. A. et al. Interactions between serotypes of dengue highlight epidemiological impact of cross-immunity. Journal of R. Soc. Interface. Vol 10: 04-14 (2013).

Reiner, R. C. et al. Time-varying, serotype-specific force of infection of dengue virus. Proc. Natl. Acad. Sci. U.S. A. 111(26): E2694-E2702 (2014).

Röst, G., Wu, J. SEIR epidemiological model with varying infectivity and infinite delay. Mathematical Biosciences and Engineering. Vol 5 (2) : 389-402 (2008).

Ruan, S. Delay Differential Equations in single species dynamics. in: Delay Differential Equations and Applications. Berlin: Springer, pp: 477-517, 2006. 
Sakdanupahp, W., Moore, E. J. A Delay Differential Equation model for Dengue Fever transmission in selected countries of South-East Asia. AIP Conference Proceedings. Vol 1148 (1): 816-819 (2009).

Sergev, V. S. Stability of solutions of Volterra Integro-differential Equations. Mathematical and Computer Modelling. 4: 1376-1394 (2007).

Van den Driessche, P. Some epidemiological Models with delays. In Differential Equations and Application to biology and to Industry. World Scientific. 507-520 (1996).

Van den Driessche, P., Zou, X. Modeling relapse in infectious diseases. Math. Biosciences. 207: 89-103 (2007).

Van den Driessche, P., Wang, L., Zou, X. Modeling diseases with latency and relapse. Mathematical Biosciences and Engineering. Vol 4 (2): 205-219 (2007).

Van den Driessche, P., Watmough, J. Further Notes on the Basic Reproduction Number. Mathematical Epidemiology. 159-178 (2008).

VinodKumar, C. S., et al. Episode of coexisting infections with multiple dengue virus serotypes in central Karnataka, India . Journal of Infection and Public Health. Vol 6 : 302-306 (2013).

Wang, J., Takeuchi, Y. A multi-group SVEIR epidemic model with distributed delay and vaccination. Int. Journal of Biomathematics. Vol 5 (3):1260001 (2012).

Wang, L., Li, Y., Pang, L. Dynamics Analysis of an Epidemiological Model with media impact and two delays. Mathematical Problems in Engineering. Vol. 2016, Article ID 1598932 (2016).

Wang, L., Z., Zhang X. Global dynamics of an SVEIR epidemic model with distributed delay and nonlinear incidence. Applied Mathematics and Computation. Vol 284: 47-65 (2016).

World Health Organization (WHO). https://www.who.int/news-room/fact-sheets/ detail/dengue-and-severe-dengue Accessed in December, 2018.

World Health Organization. Dengue: Guidelines for Diagnosis, Treatment, Prevention and Control. New edition. World Health Organization (2009).

Xiao, Y., Zou, X. On latencies in malaria infections and their impact on the disease dynamics. Math. Biosci. Eng. Vol 10 (2): 463-481 (2013).

Xu, J., Geng, Y. Zhou, Y. Global stability of a multi-group model with distributed delay and vaccination. Mathematical Methods in the Applied Sciences. Vol 40 : 1475-1486 (2017). 